囯 
FACULTÉ DES ÉTUDES SUPÉRIEURES

ET POSTOCTORALES
III

uOttawa

L'Université canadienne Canada's university
FACULTY OF GRADUATE AND POSDOCTORAL STUDIES

\section{Garrett Parsons}

AUTEUR DE LA THESE / AUTTHOR OF THESIS

M.Sc. (System Science) GRADE/DEGREE

School of System Science

FACULTE, ECOLE, DEPARTEMENT / FACÜLTYY, SCHOOL, DEPARTMENT

An Investigation of Adaptive Acquisition Techniques for Planar Near-Field Antenna Measurements

TITRE DE LA THESE / TITLE OF THESIS

Derek McNamara

DIRECTEUR (DIRECTRICE) DE LA THESE/THESIS SUPERVISOR

CO-DIRECTEUA (CO-DIRECTRICE) DE LA THESE I THESIS CO-SUPERVISOA

EXAMINATEURS (EXAMINATRICES) DE LA THĖSE / THESIS EXAMINERS

Emad Gad

Mustapha Yagoub

Gary W. Slater

Le Doyen de la Faculté des études superieures et postdoctorales / Dean of the Faculty of Graduate and Postdoctoral Studies 


\title{
AN INVESTIGATION OF ADAPTIVE ACQUISITION TECHNIQUES FOR PLANAR NEAR-FIELD ANTENNA MEASUREMENTS
}

\author{
by
}

\section{Garrett Parsons}

A thesis submitted to the

Faculty of Graduate and Postdoctoral Studies

in partial fulfillment of the requirements for the degree of

\section{Master of Science \\ in Systems Science}

(OGarrett Parsons, Ottawa, Canada, 2009 
Library and Archives Canada

\section{Published Heritage} Branch

395 Wellington Street Ottawa ON K1A ON4 Canada
Bibliotheqque et

Archives Canada

Direction du

Patrimoine de l'édition

395 , rue Wellington

Ottawa ON K1A ON4

Canada
Yourfile Votre reférence

ISBN: 978-0-494-61185-2

Our file Notre reférence

ISBN: 978-0-494-61185-2
NOTICE:

The author has granted a nonexclusive license allowing Library and Archives Canada to reproduce, publish, archive, preserve, conserve, communicate to the public by telecommunication or on the Internet, loan, distribute and sell theses worldwide, for commercial or noncommercial purposes, in microform, paper, electronic and/or any other formats.

The author retains copyright ownership and moral rights in this thesis. Neither the thesis nor substantial extracts from it may be printed or otherwise reproduced without the author's permission.
AVIS:

L'auteur a accordé une licence non exclusive permettant à la Bibliothèque et Archives Canada de reproduire, publier, archiver, sauvegarder, conserver, transmettre au public par télécommunication ou par l'Internet, prêter, distribuer et vendre des thèses partout dans le monde, à des fins commerciales ou autres, sur support microforme, papier, électronique et/ou autres formats.

L'auteur conserve la propriété du droit d'auteur et des droits moraux qui protège cette thèse. $\mathrm{Ni}$ la thèse ni des extraits substantiels de celle-ci ne doivent être imprimés ou autrement reproduits sans son autorisation.
In compliance with the Canadian Privacy Act some supporting forms may have been removed from this thesis.

While these forms may be included in the document page count, their removal does not represent any loss of content from the thesis.
Conformément à la loi canadienne sur la protection de la vie privée, quelques formulaires secondaires ont été enlevés de cette thèse.

Bien que ces formulaires aient inclus dans la pagination, il n'y aura aucun contenu manquant. 


\begin{abstract}
This thesis presents an adaptive technique that uses feedback to provide practical information during a planar near-field measurement scan. The feedback is used to decide with certainty when to terminate the planar near-field measurement process.

The developed adaptive planar near-field method utilizes a rectangular spiral-type acquisition pattern to acquire the near-field measurements. At the end of each scan iteration, a set of decision functions are rapidly calculated which provide a quality measurement of the resulting far-zone transformation. The decision functions are based on the far-zone radiated patterns, directivity, and the fractional plane wave spectrum error.

The decision functions were evaluated using actual planar near-field data set of five different antennas. These experiments have identified those decision functions that are directly related to antenna performance measures and would allow for the termination of the planar near-field test based on a set of relevant stopping conditions.
\end{abstract}


First and foremost I would like to thank Dr. Derek McNamara for his support throughout the research and writing process, and especially for his help with Section 2.5 of this thesis. I would also like to thank Dr. Daniël Janse van Rensburg for providing his expertise on the planar near-field measurement process.

Finally, to my parents and my wife Krisann, to whom I can finally answer the reoccurring question of late with, "Yes, I have finally finished." 


\section{TABLE OF CONTENTS}

CHAPTER 1 - INTRODUCTION .................................................................................... 1

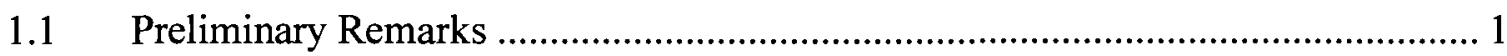

$1.2 \quad$ The Near-Field Antenna Measurement Concept ..................................................... 2

1.3 Desired Improvements in Planar Near-Field Testing ........................................... 4

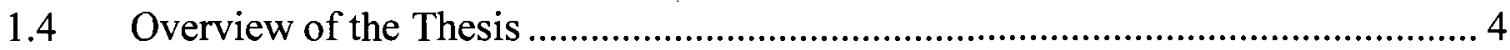

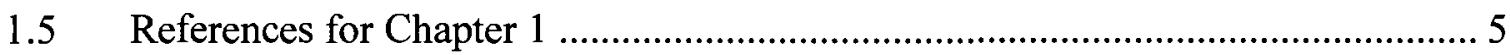

CHAPTER 2 - FUNDAMENTALS OF EXISTING PLANAR NEAR-FIELD

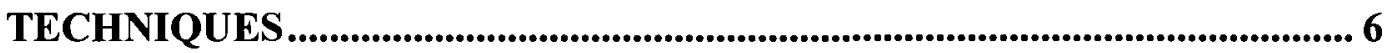

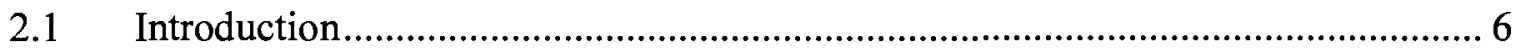

2.2 Fundamental Expressions for the Planar Near-Field to Far-Field Transformation

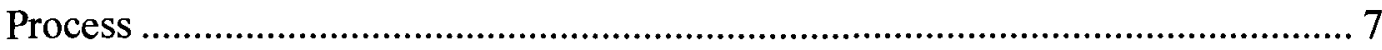

2.3 Implementation Details of the Fourier Transform ............................................... 11

2.3.1 The Discrete Fourier Transform ....................................................................... 11

2.3.2 Use of the Fast Fourier Transform ...................................................................... 13

2.3.3 Implementation of the Far-Field Transformation ............................................. 14

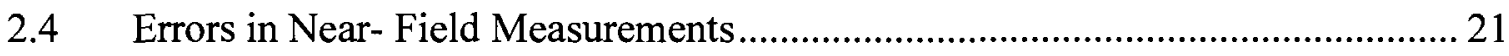

2.5 Enhancements to Planar Near-Field Measurement Techniques ........................... 24

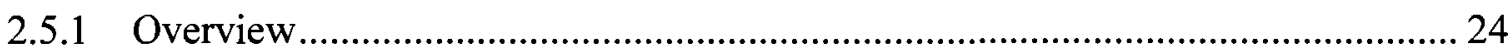

2.5.2 Near-Field Data Re-Computation Over a Plane Closer to the AUT ..................... 26

2.5.3 Near-Field Data Selection over the Scan Plane ............................................... 29

2.5.4 Near-Field Data Extrapolation over the Scan Plane ……................................... 32

2.5.5 Near-Field Data Reconstruction over the Scan Plane .......................................... 32 


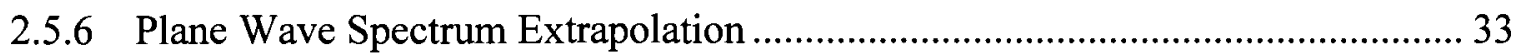

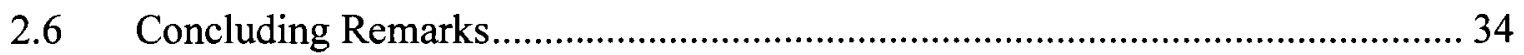

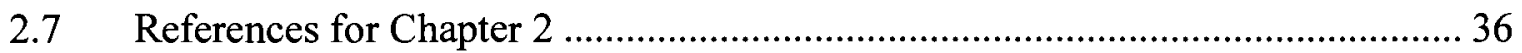

CHAPTER 3 - THE ADAPTIVE ACQUISTION TECHNIQUE ..............................41

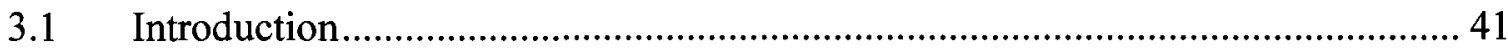

3.2 The Adaptive Acquisition Concept................................................................... 41

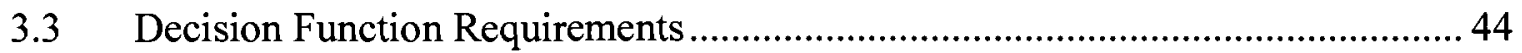

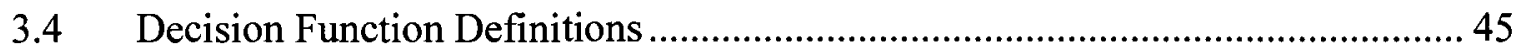

3.4.1 DFE1 - Average of E-Field Differences ........................................................... 46

3.4.2 DFE2 - Maximum of E-Field Differences ………………................................ 53

3.4.3 DFE3 - Difference of E-Fields at a Defined Point ..............................................53

3.4.4 DFD1 - Average Directivity Differences ………........................................... 54

3.4.5 DFD2 - Maximum of Directivity Differences.................................................... 58

3.4.6 DFD3 - Difference of Directivity at a Defined Point.......................................... 59

3.4.7 DFN1 - Average of Fractional Plane Wave Spectrum Error ................................ 59

3.4.8 DFN2 - Maximum of Fractional Plane Wave Spectrum Error ............................ 68

3.4.9 DFN3 - Fractional Plane Wave Spectrum Error at a Defined Point ..................... 69

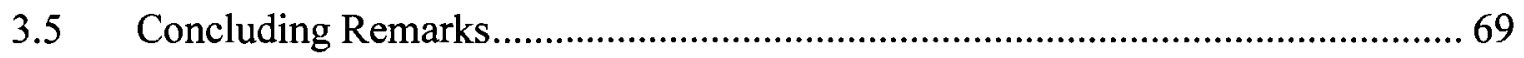

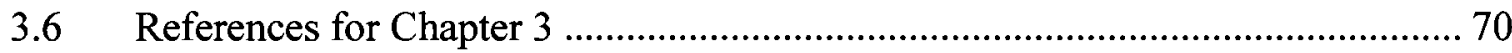

CHAPTER 4 - DATA SETS USED TO EVALUATE DECISION FUNCTIONS... 71

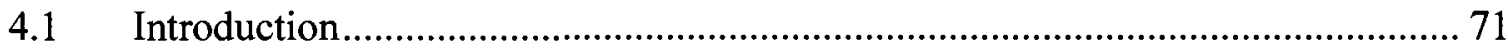

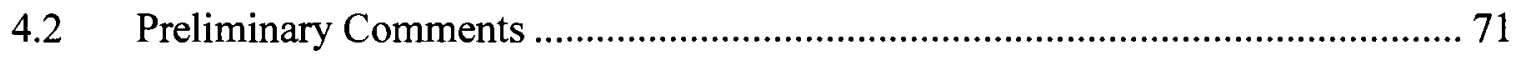

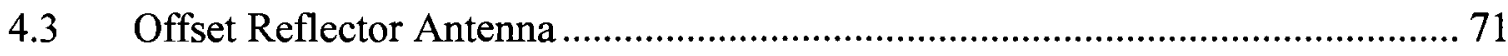




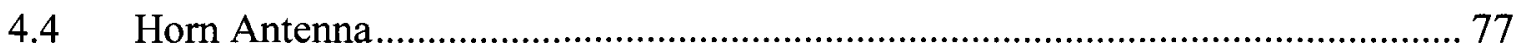

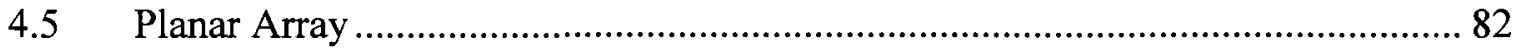

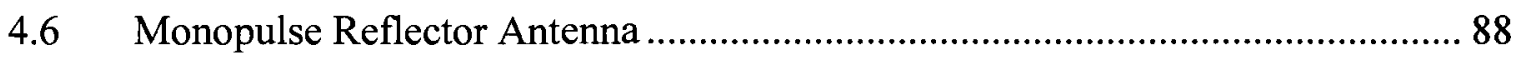

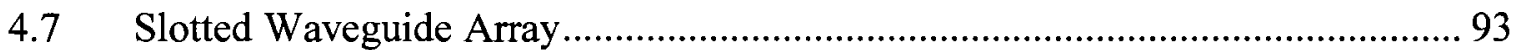

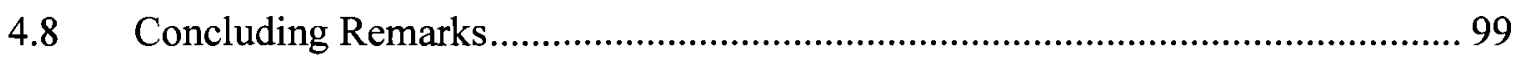

CHAPTER 5 - COMPARATIVE STUDY OF DECISION FUNCTIONS FOR ADAPTIVE PLANAR NEAR-FIELD TECHNIQUES ................................... 100

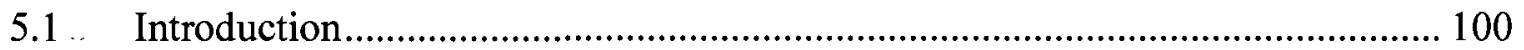

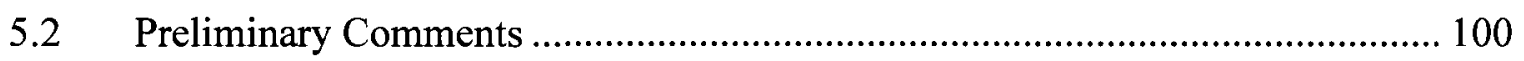

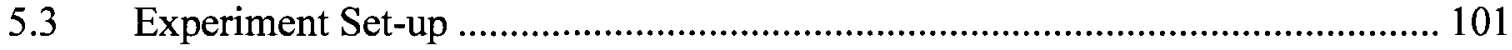

5.4 Analysis of Decision Functions DFEn

5.4.1 Analysis of Decision Function DFE1 ………................................................. 104

5.4.2 Analysis of Decision Function DFE2 ……….............................................. 107

5.4.3 Analysis of Decision Function DFE3 ………….............................................. 111

5.5 Analysis of Decision Function DFDn …………........................................... 114

5.5.1 Analysis of Directivity versus Scan Size ...................................................... 114

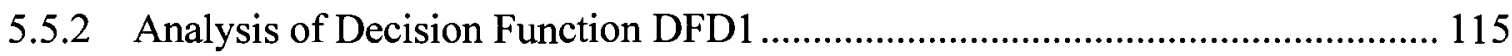

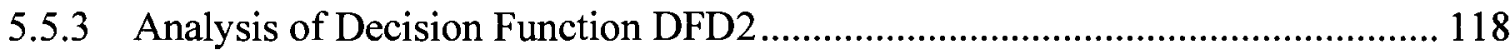

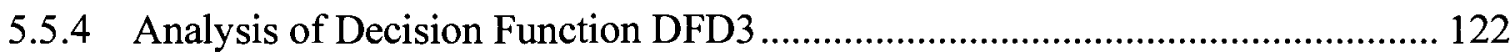

5.6 Analysis of Decision Functions DFNn …..................................................... 125

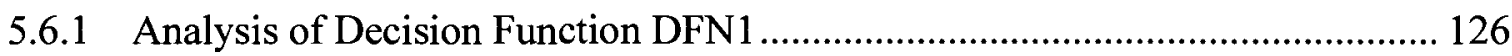

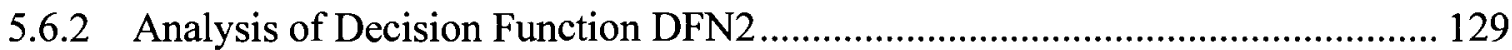

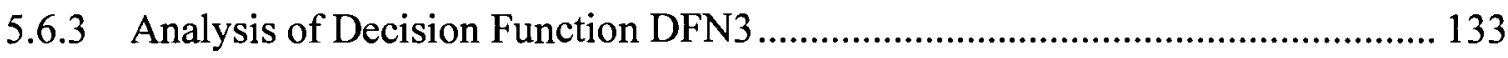


5.7 Analysis of Cross-Polarized Decision Functions................................................ 136

5.7.1 Analysis of Cross-Polarized Decision Function DFEn …………………......... 136

5.7.2 Analysis of Cross-Polarized Decision Function DFD n .................................... 138

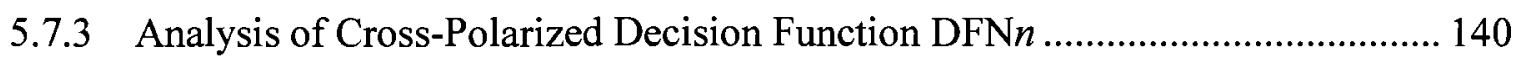

5.8 Conclusions on the Selection of Decision Functions.......................................... 142

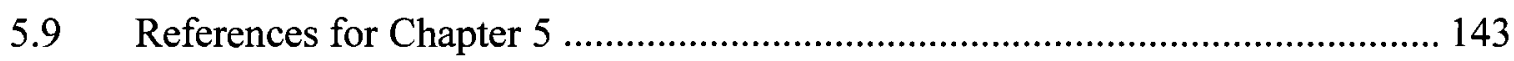

CHAPTER 6 - CONCLUSIONS......................................................................................... 144

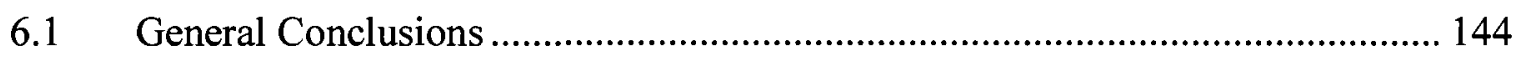

Appendix A: Simpson's Numerical Integration MATLAB Function ........................ 146

Appendix B: Examination of DFNn Nulls ................................................................... 150 


\section{LIST OF FIGURES}

Figure 1.2-1: Schematic diagram of an archetypal planar near-field scanning system. (Adapted from a diagram courtesy of NSI Inc.) ……........................................... 3

Figure 2.2-1 : Sketch Showing Physical AUT and PNF Scan Area Data Points............... 8

Figure 2.2-2: Simulated $17 \times 17$ PNF data with a 0.5 wavelength spacing, in $\mathrm{dB}$........... 19

Figure 2.2-3: Simulated 17x17 PNF data zero-padded to $32 \times 32$, in $\mathrm{dB} \ldots \ldots \ldots \ldots \ldots \ldots \ldots \ldots \ldots . . . . . . . .19$

Figure 2.2-4: Far-Field transformation of zero-padded input data generated using a direct evaluation of expression (2.3.3-6), in $\mathrm{dB}$. This is the 'expected' result. .............. 20

Figure 2.2-5: Far-Field transformation of expression (2.3.3-6) using the IFFT2 function in MATLAB on the zero-padded input data, in $\mathrm{dB}$. 20

Figure 2.2-6: Far-field transformation of zero-padded input data using the FFTSHIFT and IFFT2 functions in MATLAB to evaluate expression (2.3.3-9), in dB. This now matches the 'expected' result in Figure 2.2-4............................................... 21

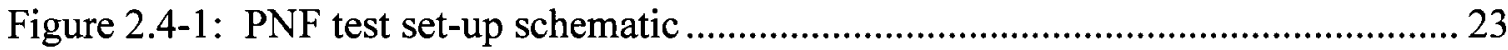

Figure 3.2-1 Sketch showing $5 \times 5$ element array (+), possible sampling points (o), and probe paths after $(n-1)^{\text {th }}(--)$ and $n^{\text {th }}(--Z)$ iterations. 43

Figure 3.4-1: Effect of $P_{D F E, c o}$ on the calculated far-zone E-field for the $\phi=0$ cut of a Horn antenna. The left image does not have a lower limit applied, while in the right image $P_{D F E, c o}=-50 \mathrm{~dB}$ 49

Figure 3.4-2: Effect of $w_{c o}\left(\theta_{i}, \phi_{j}\right)$ on the far-zone E-field of a Horn antenna. In the left image, no weighting function has been applied, while in the right image $\theta_{c o, \text { min }}=$ $-30^{\circ}$ and $\theta_{c o, \max }=30^{\circ}$ 51 
Figure 3.4-3: Plot showing DFEn values required for an uncertainty, $\pm \Delta_{d B}$, at a particular

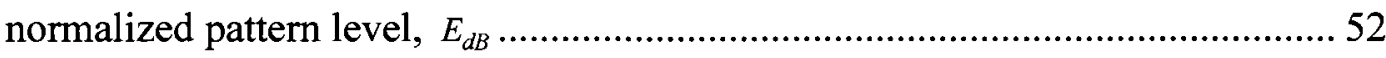

Figure 3.4-4: $\gamma_{\max }$ for DFN1. The maximum acute angle occurs once the aperture has been scanned and when $\mathrm{a}$ is the largest AUT aperture dimension. 61

Figure 3.4-5: Sketch showing contour integral in the numerator of expressions 3.4.7-1 and 3.4.7-2 62

Figure 3.4-6: Error in directivity based on evaluation of 3.4.7-9, for a given fractional error in plane wave spectrum 66

Figure 4.3-1: Near-field $\left|E_{\mathrm{x}}(x, y)\right|$ sampled over the scan plane for the offset reflector antenna, normalized to $E_{y}$, in $\mathrm{dB}$. 73

Figure 4.3-2: Normalised near-field $\left|E_{\mathrm{y}}(x, y)\right|$ sampled over the scan plane for the offset reflector antenna, in $\mathrm{dB}$ 73

Figure 4.3-3: Co (o) and cross (x) polarized directivity of the offset reflector antenna based on near-field scan size. 74

Figure 4.3-4: Normalized far-field $\left|E_{\mathrm{co}}(\theta, \phi)\right|$ of the offset reflector antenna for pattern cut $\phi=0^{\circ}$ 74

Figure 4.3-5: Far-field $\left|E_{\mathrm{cr}}(\theta, \phi)\right|$ of the offset reflector antenna for pattern cut $\phi=0^{\circ}$, normalized to $\left|E_{\mathrm{co}}(\theta, \phi)\right|$ 75

Figure 4.3-6: Normalized far-field $\left|E_{\mathrm{co}}(\theta, \phi)\right|$ of the offset reflector antenna for pattern $\operatorname{cut} \phi=90^{\circ}$ 75

Figure 4.3-7: Far-field $\left|E_{\mathrm{cr}}(\theta, \phi)\right|$ of the offset reflector antenna for pattern cut $\phi=90^{\circ}$, normalized to $\left|E_{\mathrm{co}}(\theta, \phi)\right|$ 76 
Figure 4.3-8: Rule-of-thumb valid far-zone angle off broadside for the offset reflector antenna. The vertical line shows the AUT aperture

Figure 4.4-1: Normalized near-field $\left|E_{\mathrm{x}}(x, y)\right|$ sampled over the scan plane for the horn antenna, in $\mathrm{dB}$ 78

Figure 4.4-2: Near-field $\left|E_{\mathrm{y}}(x, y)\right|$ sampled over the scan plane for the horn antenna, normalized to $E_{\mathrm{x}}$, in $\mathrm{dB}$ 79

Figure 4.4-3: Co (o) and cross (x) polarized directivity of the horn antenna based on near-field scan size. 79

Figure 4.4-4: Normalized far-field $\left|E_{\mathrm{co}}(\theta, \phi)\right|$ of the horn antenna for pattern cut $\phi=0^{\circ} 80$

Figure 4.4-5: Far-field $\left|E_{\mathrm{cr}}(\theta, \phi)\right|$ of the horn antenna for pattern cut $\phi=0^{\circ}$, normalized to $\left|E_{\mathrm{co}}(\theta, \phi)\right|$ 80

Figure 4.4-6: Normalized far-field $\left|E_{\mathrm{co}}(\theta, \phi)\right|$ of the horn antenna for the pattern cut $\phi=$ $90^{\circ}$ 81

Figure 4.4-7: Far-field $\left|E_{\mathrm{cr}}(\theta, \phi)\right|$ of the horn antenna for pattern cut $\phi=90^{\circ}$, normalized to $\left|E_{\mathrm{co}}(\theta, \phi)\right|$ 81

Figure 4.4-8: Rule-of-thumb valid far-zone angle off broadside for the horn antenna. The vertical line shows the AUT aperture 82

Figure 4.5-1: Near-field $\left|E_{\mathbf{x}}(x, y)\right|$ sampled over the scan plane for the planar array, normalized to $E_{\mathrm{y}}$, in $\mathrm{dB}$ 84

Figure 4.5-2: Normalized near-field $\left|E_{y}(x, y)\right|$ sampled over the scan plane for the planar array, in $\mathrm{dB}$. 84

Figure 4.5-3: Co (o) and cross (x) polarized directivity of planar array based on nearfield scan size. 85 
Figure 4.5-4: Normalized far-field $\left|E_{\mathrm{co}}(\theta, \phi)\right|$ of the planar array for pattern cut $\phi=0^{\circ} .85$

Figure 4.5-5: Far-field $\left|E_{\mathrm{cr}}(\theta, \phi)\right|$ of the planar array for pattern cut $\phi=0^{\circ}$, normalized to

$$
\left|E_{\mathrm{co}}(\theta, \phi)\right|
$$

Figure 4.5-6: Normalized far-field $\left|E_{\mathrm{co}}(\theta, \phi)\right|$ of the planar array for pattern cut $\phi=90^{\circ} 86$

Figure 4.5-7: Far-field $\left|E_{\mathrm{cr}}(\theta, \phi)\right|$ of the planar array for pattern $\operatorname{cut} \phi=90^{\circ}$, normalized

to $\left|E_{\mathrm{co}}(\theta, \phi)\right|$

Figure 4.5-8: Rule-of-thumb valid far-zone angle off broadside for the planar array. The vertical line shows the AUT aperture 88

Figure 4.6-1: Near-field $\left|E_{\mathrm{x}}(x, y)\right|$ sampled over the scan plane for the monopulse reflector antenna, normalized to $E_{\mathrm{y}}$, in $\mathrm{dB}$

Figure 4.6-2: Normalized near-field $\left|E_{\mathrm{y}}(x, y)\right|$ sampled over the scan plane for the monopulse reflector antenna, in $\mathrm{dB}$ 90

Figure 4.6-3: Co (o) and cross (x) polarized directivity of monopulse reflector antenna based on near-field scan size 90

Figure 4.6-4: Normalized far-field $\left|E_{\mathrm{co}}(\theta, \phi)\right|$ of the monopulse reflector antenna for pattern cut $\phi=0^{\circ}$ 91

Figure 4.6-5: Far-field $\left|E_{\mathrm{cr}}(\theta, \phi)\right|$ of the monopulse reflector antenna for pattern cut $\phi=$ $0^{\circ}$, normalized to $\left|E_{\mathrm{co}}(\theta, \phi)\right|$ 91

Figure 4.6-6: Normalized far-field $\left|E_{\mathrm{co}}(\theta, \phi)\right|$ of the monopulse reflector antenna for pattern $\operatorname{cut} \phi=90^{\circ}$ 92

Figure 4.6-7: Far-field $\left|E_{\mathrm{cr}}(\theta, \phi)\right|$ of the monopulse reflector antenna for pattern cut $\phi=$ $90^{\circ}$, normalized to $\left|E_{\mathrm{co}}(\theta, \phi)\right|$ 
Figure 4.6-8: Rule-of-thumb valid far-zone angle off broadside for the monopulse reflector antenna. The vertical line shows the AUT aperture 93

Figure 4.7-1: Normalized near-field $\left|E_{\mathrm{x}}(x, y)\right|$ sampled over the scan plane for the slotted waveguide array, in $\mathrm{dB}$ 95

Figure 4.7-2: Near-field $\left|E_{\mathrm{y}}(x, y)\right|$ sampled over the scan plane for the slotted waveguide array, normalized to $E_{\mathrm{x}}$, in $\mathrm{dB}$ 95

Figure 4.7-3: Co (o) and cross (x) polarized directivity of slotted waveguide array based on near-field scan size. 96

Figure 4.7-4: Normalized far-field $\left|E_{\mathrm{co}}(\theta, \phi)\right|$ of the slotted waveguide array for pattern $\operatorname{cut} \phi=0^{\circ}$ 96

Figure 4.7-5: Far-field $\left|E_{\mathrm{cr}}(\theta, \phi)\right|$ of the slotted waveguide array for pattern cut $\phi=0^{\circ}$, normalized to $\left|E_{\mathrm{co}}(\theta, \phi)\right|$ 97

Figure 4.7-6: Normalized far-field $\left|E_{\mathrm{co}}(\theta, \phi)\right|$ of the slotted waveguide array for pattern cut $\phi=90^{\circ}$ 97

Figure 4.7-7: Far-field $\left|E_{\mathrm{cr}}(\theta, \phi)\right|$ of the slotted waveguide array for pattern cut $\phi=90^{\circ}$, normalized to $\left|E_{\mathrm{co}}(\theta, \phi)\right|$ 98

Figure 4.7-8: Rule-of-thumb valid far-zone angle off broadside for the slotted waveguide array. The vertical line shows the AUT aperture. 99

Figure 5.4-1: Co-polarized component of DFE1 for the offset reflector antenna ......... 105

Figure 5.4-2: Co-polarized component of DFE1 for the horn antenna........................... 105

Figure 5.4-3: Co-polarized component of DFE1 for the planar array ........................... 106

Figure 5.4-4: Co-polarized component of DFE1 for the monopulse reflector antenna. 106 Figure 5.4-5: Co-polarized component of DFE1 for the slotted waveguide array ........ 107 
Figure 5.4-6: Co-polarized component of DFE2 for the offset reflector antenna ......... 108

Figure 5.4-7: Co-polarized component of DFE2 for the horn antenna........................ 109

Figure 5.4-8: Co-polarized component of DFE2 for the planar array ........................... 109

Figure 5.4-9: Co-polarized component of DFE2 for the monopulse reflector antenna . 110

Figure 5.4-10: Co-polarized component of DFE2 for the slotted waveguide array ...... 110

Figure 5.4-11: Co-polarized component of DFE3 for the reflector antenna................... 111

Figure 5.4-12: Co-polarized component of DFE3 for the horn antenna........................ 112

Figure 5.4-13: Co-polarized component of DFE3 for the planar array ......................... 112

Figure 5.4-14: Co-polarized component of DFE3 for the monopulse reflector antenna 113

Figure 5.4-15: Co-polarized component of DFE3 for the slotted waveguide array ...... 113

Figure 5.5-1: Co-polarized component of DFD1 for the offset reflector antenna ......... 116

Figure 5.5-2: Co-polarized component of DFD1 for the horn antenna ....................... 116

Figure 5.5-3: Co-polarized component of DFD1 for the planar array .......................... 117

Figure 5.5-4: Co-polarized component of DFD1 for the monopulse reflector antenna. 117

Figure 5.5-5: Co-polarized component of DFD1 for the slotted waveguide array ........ 118

Figure 5.5-6: Co-polarized component of DFD2 for the offset reflector antenna ......... 120

Figure 5.5-7: Co-polarized component of DFD2 for the horn antenna ........................ 120

Figure 5.5-8: Co-polarized component of DFD2 for the planar array .......................... 121

Figure 5.5-9: Co-polarized component of DFD2 for the monopulse reflector antenna. 121

Figure 5.5-10: Co-polarized component of DFD2 for the slotted waveguide array ...... 122

Figure 5.5-11: Co-polarized component of DFD3 for the offset reflector antenna ....... 123

Figure 5.5-12: Co-polarized component of DFD3 for the horn antenna ....................... 123

Figure 5.5-13: Co-polarized component of DFD3 for the planar array ......................... 124 
Figure 5.5-14: Co-polarized component of DFD3 for the monopulse reflector antenna124

Figure 5.5-15: Co-polarized component of DFD3 for the slotted waveguide array ...... 125

Figure 5.6-1: Y-component of DFN1 for the offset reflector antenna........................... 127

Figure 5.6-2: X-component of DFN1 for the horn antenna .......................................... 127

Figure 5.6-3: Y-component of DFN1 for the planar array............................................ 128

Figure 5.6-4: Y-component of DFN1 for the monopulse reflector antenna .................. 128

Figure 5.6-5: X-component of DFN1 for the slotted waveguide array.......................... 129

Figure 5.6-6: Y-component of DFN2 for the offset reflector antenna........................... 130

Figure 5.6-7: X-component of DFN2 for the horn antenna ....................................... 131

Figure 5.6-8: Y-component of DFN2 for the planar array........................................... 131

Figure 5.6-9: Y-component of DFN2 for the monopulse reflector antenna ................... 132

Figure 5.6-10: X-component of DFN2 for the slotted waveguide array........................ 132

Figure 5.6-11: Y-component of DFN3 for the offset reflector antenna......................... 133

Figure 5.6-12: X-component of DFN3 for the horn antenna ........................................ 134

Figure 5.6-13: Y-component of DFN3 for the planar array....................................... 134

Figure 5.6-14: Y-component of DFN3 for the monopulse reflector antenna ................ 135

Figure 5.6-15: X-component of DFN3 for the slotted waveguide array........................ 135

Figure 5.7-1: Cross-polarized component of DFE1 for the offset reflector antenna ..... 137

Figure 5.7-2: Cross-polarized component of DFE2 for the offset reflector antenna .... 137

Figure 5.7-3: Cross-polarized component of DFE3 for the offset reflector antenna ..... 138

Figure 5.7-4: Cross-polarized component of DFD1 for the offset reflector antenna..... 139

Figure 5.7-5: Cross-polarized component of DFD2 for the offset reflector antenna..... 139

Figure 5.7-6: Cross-polarized component of DFD3 for the offset reflector antenna..... 140 
Figure 5.7-7: X-component of DFN1 for the offset reflector antenna........................... 141

Figure 5.7-8: X-component of DFN2 for the offset reflector antenna........................... 141

Figure 5.7-9: X-component of DFN3 for the offset reflector antenna.......................... 142 
Table 3.4-1: DFE $n$ values required for an uncertainty, $\pm \Delta_{d B}$, at a normalized pattern level, $E_{d B}$ 52

Table 3.4-2: Error in directivity based on evaluation of (3.4.7-9), for a given fractional error in plane wave spectrum ......................................................................... 65

Table 4.3-1: PNF scan information for the offset reflector antenna ............................... 72

Table 4.4-1: PNF scan information for the horn antenna ………………….................. 78

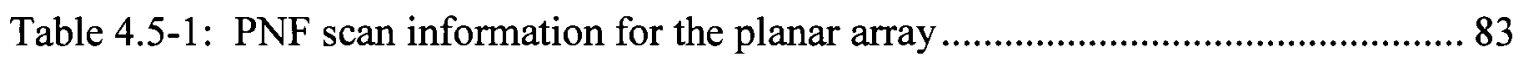

Table 4.6-1: PNF scan information for the monopulse reflector antenna......................... 89

Table 4.7-1: PNF scan information for the slotted waveguide array .............................. 94

Table 5.6-1: Summary of principle near-field components for antenna types .............. 126 


\section{CHAPTER 1 - INTRODUCTION}

\subsection{Preliminary Remarks}

In spite of uncertainties in the communications industry in recent years, no doubt there will be continued technology development to provide diverse services that require higher data rates and allow user mobility. Mobility at once means that antennas will continue to be key components. Service diversity will mean that a single antenna structure might have to provide slightly different characteristics for each of a number of roles (i.e. have multi-functionality). This implies multiple polarizations and beam ports, as well as electronically controllable beam characteristics with embedded intelligence. Increased data rates demand antennas with wider operating frequency bands, and so spectrum has been allocated at millimetre-wave (mm-wave) frequencies. There will have to be significant advances in antenna configurations, and methods for their design, to ensure the success of future systems. The antennas must satisfy more demanding requirements, yet at a cost suitable for commercial applications. These comments apply to antenna configurations for both terrestrial and satellite applications.

As antenna performance complexity increases, so too does the suite of measurements that are needed to confirm whether a given antenna indeed satisfies the required performance. Such more elaborate testing at once implies that the measurements will be more time-consuming. It is important to realize that it is not the speed with which electronic instrumentation is able to operate that is of concern here. When it comes to antenna testing this aspect occupies a negligible fraction of the time involved. Rather, it is the acquisition time related to the three-dimensional movement of the antenna under test 
(AUT), or the acquisition probe, during measurement that is the principal contributor. While the time taken to complete a measurement might be of little consequence in a research environment, once antennas are being manufactured on a production line time is of the essence. In fact, it is believed by many that "in developing products and systems, testing, and not design, is usually the more expensive, time-consuming, and difficult activity" [1].

\subsection{The Near-Field Antenna Measurement Concept}

There is a large amount of literature on antenna measurements [2], [3], [4]. Such measurements can be broadly classified into far-field measurement techniques and nearfield measurement techniques. It is most often the far-field radiation pattern (and quantities derived from it) of an antenna that we are eventually interested in. Far-field measurement techniques require the AUT and the acquisition probe to be separated a distance sufficiently large that the electromagnetic fields radiated by the AUT are locally plane at the location of the probe. Far-field measurement techniques provide the far-field radiation patterns directly. Near-field measurement techniques use an acquisition probe that can in principle be located at any distance from the AUT; theoretical formulations (we will simply call these far-field transformations) based on fundamental electromagnetic theory are then used to predict the far-field radiation patterns from the measured data acquired in the near-field of the AUT.

Near-field measurement techniques can in turn be divided into three classes: planar near-field testing, cylindrical near-field testing and spherical near-field testing. The classes are named according to the shape of the surface surrounding the AUT over 
which the acquisition probe samples the data. The case of planar near-field testing is shown in Figure 1.2-1. In this case the far-field patterns are [5] in essence twodimensional Fourier transforms of the near-field distribution over a planar surface in front of the AUT. We will refer to the plane over which such samples are measured (in amplitude and phase) as the scan plane. Samples (which we will call data points) are of course only taken over a finite portion of the scan plane; this we will refer to as the scan area. A specific AUT will have a particular physical aperture. The larger the physical aperture of the AUT the larger the scan area needs to be.

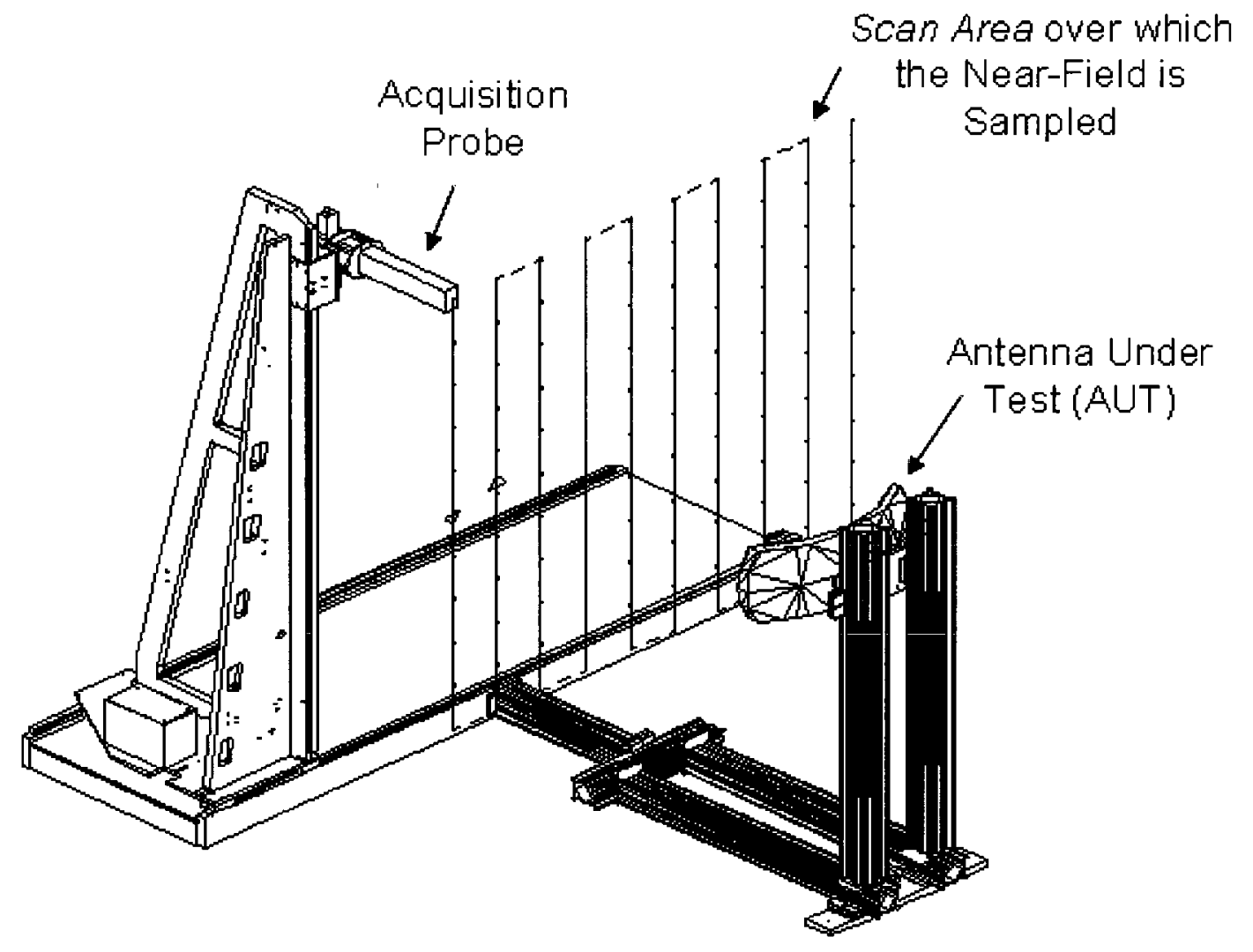

Figure 1.2-1: Schematic diagram of an archetypal planar near-field scanning system. (Adapted from a diagram courtesy of NSI Inc.) 


\subsection{Desired Improvements in Planar Near-Field Testing}

The aim of this research was to explore and develop methods for reducing the data capture burden in antenna planar near-field (PNF) measurements. In order to do this we must be able to keep to a minimum the scan area (that is, the number of required data points in the scan plane $^{1}$ ) for a given antenna physical aperture. Although at the start of the research described in this thesis we conjectured several possible ways of doing this, it soon became clear that an adaptive approach was the overwhelmingly superior one. We came to this conclusion early on, as soon as it was recognized that the time to compute the far-field transformation (using the fast Fourier transform) was for all practical purposes negligible compared to the data acquisition time. It has therefore been the only one pursued in this research, and represents the first time that continuous feedback (during the actual measurement process) has been used in determining when to end the scan area as soon as possible, thereby minimizing the testing time.

\subsection{Overview of the Thesis}

Chapter 2 provides a review of the essentials of the PNF measurement process needed to properly describe the ideas and contributions of this thesis. It also provides an opportunity to introduce the notation that will be used in later chapters. Furthermore, some implementation details of PNF measurements that are obviously used by others, but do not appear to be explicitly stated elsewhere, are provided in this chapter. In Chapter 3 we motivate and propose an adaptive algorithm aimed at reducing overall PNF testing

\footnotetext{
${ }^{1}$ Sampling theorems dictate the spacing between data points. Thus these two ways of stating the problem are equivalent.
} 
time. We describe how the adaptivity is achieved by the processing of the measured data, and forming what we have called decision functions, while the data is still being sampled. Several possible decision functions are defined in Section 3.4. Evaluation of the adaptive algorithm necessitates the use of measured PNF data and details of the data sets used in this thesis are thus presented in Chapter 4. This is followed in Chapter 5 by a study of the effectiveness of the decision functions in reducing PNF test times. Finally, some general conclusions are reached in Chapter 6, and the research reported herein put into perspective.

\subsection{References for Chapter 1}

[1] P.D.T.O'Connor, Editorial, IEEE Spectrum, pp. 18, Jul. 2001

[2] E.S.Gillespie. "Measurement of Antenna Radiation Characteristics on Far-Field Ranges" in Antenna Handbook, vol. 4. Y.T.Lo \& S.W.Lee, Ed. New York: Van Nostrand Reinhold, 1993, Ch32.

[3] J.Appel-Hansen, "Near-Field Far-Field Antenna Measurements" in Antenna Handbook, vol. 4. Y.T.Lo \& S.W.Lee, Ed. New York: Van Nostrand Reinhold, 1993 , Ch33.

[4] J.Appel-Hansen, E.S.Gillespie, T.G.Hickman \& J.D.Dysion, "Antenna Measurements" in The Handbook of Antenna Design, vol. 2. A.W.Rudge, K.Milne, A.D.Olver \& P.Knight Ed. London: Peter Peregrinus Ltd., 1986, Ch8.

[5] J.J.H.Wang, "An examination of the theory and practices of planar near-field measurement", IEEE Transactions on Antennas \& Propagation, vol. 36, no. 6, pp. 746-753, Jun. 1988.

[6] D.Slater, Near-Field Antenna Measurements. Boston: Artech House Inc., 1991).

[7] S.F. Gregson, J. McCormic, \& C. Parini. Principles of Planar Near-Field Antenna Measurements. London, UK: The Institution of Engineering and Technology, 2007 


\section{CHAPTER 2 - FUNDAMENTALS OF EXISTING PLANAR NEAR-FIELD TECHNIQUES}

\subsection{Introduction}

In order to describe the development of the adaptive technique for PNF measurements it is necessary to delve into the mathematical analysis on which the method is based. In Section 2.2 we describe the theoretical basis of PNF measurements. The manner in which certain PNF transformation integrals are evaluated in practice is described in Section 2.3, along with some details on the manner in which this is actually implemented in the present thesis.

No measurement technique is perfect. Section 2.4 briefly describes two sources of error in PNF measurements that we will need to refer to in this thesis, in particular socalled truncation errors. The latter kind of error arises due to the fact that, although the theory in principle requires that we sample the near-field over an infinite plane in front of the AUT, we of course are only able to do so over a finite scan area. The near-field values are assumed to be zero outside the scan area. In later sections of the thesis we will use adaptive methods to minimize the size of the required scan area (dependent on what we actually wish to achieve from the measurement), in so doing reducing test times.

In Section 2.5 we review work that has been done by others to allow a PNF system with a given maximum scan area to be used in the testing of antennas with physical apertures that are larger than would be possible using conventional PNF approaches. We will indicate why these do not lead to test time reductions, or are otherwise not suitable, for present purposes. 
Conclusions and references for the present chapter are provided in Section 2.6 and Section 2.7 , respectively.

\subsection{Fundamental Expressions for the Planar Near-Field to Far- Field Transformation Process}

Briefly stated, the far-field of an antenna is obtained by taking the Fourier transform of the antenna's near-field over the scan plane in front of the antenna, Figure 2.2-1, and performing additional mathematical manipulations to remove the effect of the characteristics of the probe, a procedure known as probe correction.

If the measured transverse electric field values in the $z=0$ plane, expressed in volts/metre, are $E_{x}(x, y, 0)$ and $E_{y}(x, y, 0)$, then the plane wave spectrum [1] is described by $\bar{A}\left(k_{x}, k_{y}\right)$, whose components are

$$
\begin{aligned}
& A_{x}\left(k_{x}, k_{y}\right)=\frac{1}{2 \pi} \int_{-\infty}^{\infty} \int_{-\infty}^{\infty} E_{x}(x, y, 0) e^{j\left(k_{x} x+k_{y} y\right)} d x d y \\
& A_{y}\left(k_{x}, k_{y}\right)=\frac{1}{2 \pi} \int_{-\infty}^{\infty} \int_{-\infty}^{\infty} E_{y}(x, y, 0) e^{j\left(k_{x} x+k_{y} y\right)} d x d y
\end{aligned}
$$

and 


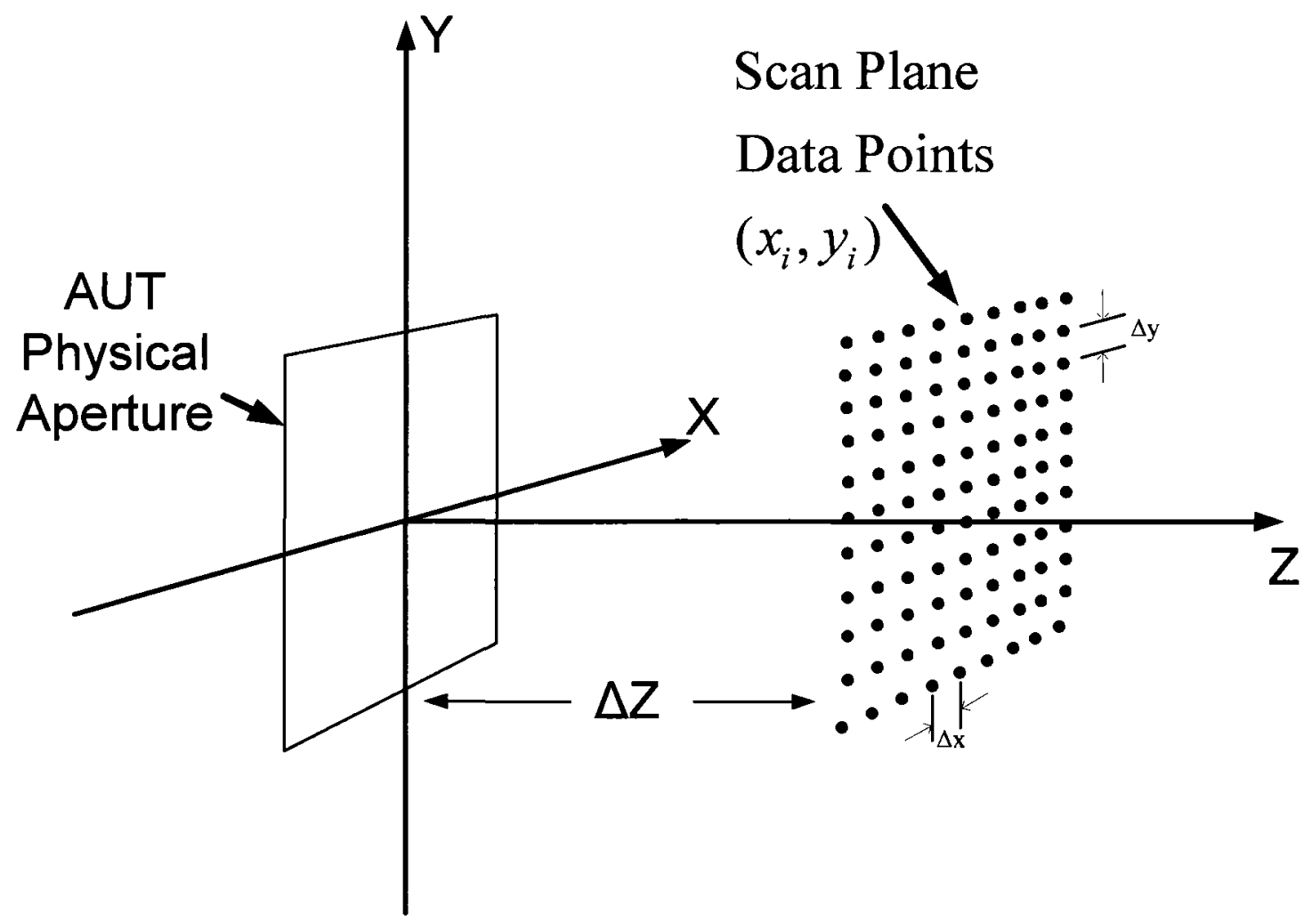

Figure 2.2-1 : Sketch Showing Physical AUT and PNF Scan Area Data Points

$$
A_{z}\left(k_{x}, k_{y}\right)=-\frac{k_{x} A_{x}\left(k_{x}, k_{y}\right)+k_{y} A_{y}\left(k_{x}, k_{y}\right)}{k_{z}}
$$

where

$$
k_{z}=\left\{\begin{array}{cc}
\sqrt{k^{2}-k_{x}^{2}-k_{y}^{2}} & \text { if } k_{x}^{2}+k_{x}^{2} \leq k^{2} \\
-j \sqrt{k_{x}^{2}+k_{y}^{2}-k^{2}} & \text { otherwise }
\end{array} .\right.
$$


If we know $A\left(k_{x}, k_{y}\right)$, we can determine fields $E_{x}\left(x_{0}, y_{0}, z_{0}\right), E_{y}\left(x_{0}, y_{0}, z_{0}\right)$ and $E_{z}\left(x_{0}, y_{0}, z_{0}\right)$ at any observation point $\left(x_{0}, y_{0}, z_{0}\right)$ in space using the inverse transformations [1], [2]

$$
\begin{aligned}
& E_{x}\left(x_{0}, y_{0}, z_{0}\right)=\frac{1}{2 \pi} \int_{-\infty}^{\infty} \int_{-\infty}^{\infty} A_{x}\left(k_{x}, k_{y}\right) e^{-j k_{z} z_{0}} e^{-j\left(k_{x} x_{0}+k_{y} y_{0}\right)} d k_{x} d k_{y} \\
& E_{y}\left(x_{0}, y_{0}, z_{0}\right)=\frac{1}{2 \pi} \int_{-\infty}^{\infty} \int_{-\infty}^{\infty} A_{y}\left(k_{x}, k_{y}\right) e^{-j k_{z} z_{0}} e^{-j\left(k_{x} x_{0}+k_{y} y_{0}\right)} d k_{x} d k_{y}
\end{aligned}
$$

and

$$
E_{z}\left(x_{0}, y_{0}, z_{0}\right)=\frac{1}{2 \pi} \int_{-\infty}^{\infty} \int_{-\infty}^{\infty} A_{z}\left(k_{x}, k_{y}\right) e^{-j k_{z} z_{0}} e^{-j\left(k_{x} x_{0}+k_{y} y_{0}\right)} d k_{x} d k_{y}
$$

If the observation point $\left(x_{0}, y_{0}, z_{0}\right)=\left(r_{0}, \theta_{0}, \phi_{0}\right)$ is in the far-zone of the antenna, with

$$
\begin{aligned}
& r_{0}=\sqrt{x_{0}^{2}+y_{0}^{2}+z_{0}^{2}} \\
& \theta_{0}=\tan ^{-1}\left\{\frac{\sqrt{x_{0}^{2}+y_{0}^{2}}}{z_{0}}\right\} \\
& \phi_{0}=\tan ^{-1}\left\{\frac{y_{0}}{x_{0}}\right\}
\end{aligned}
$$




$$
\begin{aligned}
& x_{0}=r_{0} \sin \theta_{0} \cos \phi_{0} \\
& y_{0}=r_{0} \sin \theta_{0} \sin \phi_{0}
\end{aligned}
$$

and

$$
z_{0}=r_{0} \cos \theta_{0}
$$

then asymptotic analysis shows that the above integrals simplify to [1], [2], [3], [4]

$$
\begin{aligned}
& E_{x}^{f a r}\left(x_{0}, y_{0}, z_{0}\right)=E_{x}^{f a r}\left(r_{0}, \theta_{0}, \phi_{0}\right)=j k_{z 0} A_{x}\left(k_{x 0}, k_{y 0}\right) \frac{e^{-j k r_{0}}}{r_{0}} \\
& E_{y}^{f a r}\left(x_{0}, y_{0}, z_{0}\right)=E_{y}^{f a r}\left(r_{0}, \theta_{0}, \phi_{0}\right)=j k_{z 0} A_{y}\left(k_{x 0}, k_{y 0}\right) \frac{e^{-j k r_{0}}}{r_{0}}
\end{aligned}
$$

and

$$
E_{z}^{f a r}\left(x_{0}, y_{0}, z_{0}\right)=E_{z}^{f a r}\left(r_{0}, \theta_{0}, \phi_{0}\right)=j k_{z 0} A_{z}\left(k_{x 0}, k_{y 0}\right) \frac{e^{-j r_{0}}}{r_{0}}
$$

where

$$
k_{x 0}=k \sin \theta_{0} \cos \phi_{0}
$$




$$
k_{y 0}=k \sin \theta_{0} \sin \phi_{0}
$$

and

$$
k_{z 0}=k \cos \theta_{0} .
$$

In other words, the far-zone fields are simply the plane wave spectra evaluated at the specific values of $\left(k_{x}, k_{y}, k_{z}\right)$ given immediately above. It is clear that if we specify values of $k_{x 0}$ and $k_{y 0}$, then $k_{z 0}$ can be found from $k_{z 0}=\sqrt{k^{2}-k_{x 0}^{2}-k_{y 0}^{2}}$, and then

$$
\theta_{0}=\tan ^{-1}\left\{\frac{\sqrt{k_{x 0}^{2}+k_{y 0}^{2}}}{k_{z 0}}\right\}
$$

and

$$
\phi_{0}=\tan ^{-1}\left\{\frac{k_{y 0}}{k_{x 0}}\right\} \text {. }
$$

\subsection{Implementation Details of the Fourier Transform}

\subsubsection{The Discrete Fourier Transform}

The discrete Fourier transform (DFT) is used to transform one discrete function into another, usually interpreted as from one domain (eg. time; space) into another (eg. frequency; spatial frequency). The forward DFT is expressed by 


$$
F_{k}=\sum_{n=0}^{N-1} f_{n} e^{-j \frac{2 \pi}{N} k n} \quad k=0,1, \ldots, N-1
$$

and the inverse DFT is similarly expressed by

$$
f_{n}=\frac{1}{N} \sum_{k=0}^{N-1} F_{k} e^{\frac{j 2 \pi}{N} k n} \quad n=0,1, \ldots ., N-1 .
$$

In the above expressions $\left\{f_{0}, f_{1}, f_{2}, \ldots . ., f_{N-1}\right\}$ represent the original discrete function and $\left\{F_{0}, F_{1}, F_{2}, \ldots . ., F_{N-1}\right\}$ the transformed discrete function. Expressions (2.3.1-1) and (2.3.1-2) represent a Fourier transform pair only if the input function is in fact periodic. In other words (2.3.1-2) actually gives the Fourier transform of an input function

$$
\left\{\cdots \cdots, \underline{f_{0}, f_{1}, f_{2}, \ldots \ldots, f_{N-1}}, \underline{f_{0}, f_{1}, f_{2}, \ldots ., f_{N-1}}, \underline{f_{0}, f_{1}, f_{2}, \ldots \ldots, f_{N-1}}, \ldots \ldots\right\}
$$

where the dots at each end indicate that the sequence of values $f_{0}, f_{1}, f_{2}, \ldots . ., f_{N-1}$ are repeated infinitely many times in both directions. In other words, for the N-point discrete function $f$, we have $f_{n+N}=f_{n}$. The Fourier transform (the output function) itself is also periodic, namely

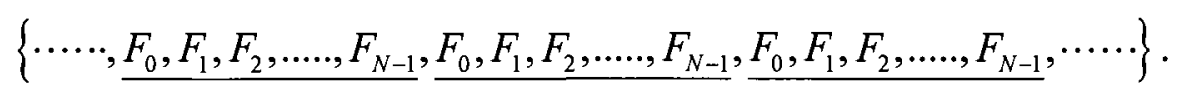




\subsubsection{Use of the Fast Fourier Transform}

The DFT can be evaluated in a very computationally efficient manor using the fast Fourier transform (FFT) algorithm. Computing a DFT of $N$ points as defined in equations (2.3.1-1) and (2.3.1-2) takes $N^{2}$ operations, whereas the FFT can calculate the same result using $N \log N$ operations. For large $N$ this is of great significance.

The authors of [5] observe that "There are many algorithms that calculate the FFT, and one of the simplest methods was discovered by Danielson and Lanczos in 1942. It was later found that these modern FFT algorithms were actually a rediscovery of efficient methods for calculating the DFT that can be traced back to Gauss in 1805". Danielson and Lanczos showed that a DFT of an input function $f_{n}, n=0,1, \ldots, N-1$, where $\mathrm{N}$ is even, can be written as the sum of two transforms of length $\mathrm{N} / 2, F_{k}^{e}$ and $F_{k}^{o}$ where $F_{k}^{e}$ is formed from the even components of $f_{n}$, and $F_{k}^{o}$ is from the odd components, namely

$$
F_{k}=\sum_{n=0}^{N-1} f_{n} e^{j 2 \pi \frac{k n}{N}}=\sum_{n=0}^{N / 2=1} f_{2 n} e^{j 2 \pi \frac{(2 n) k}{N}}+\sum_{n=0}^{N / 2-1} f_{2 n+1} e^{j 2 \pi \frac{(2 n+1) k}{N}}
$$

If we let $W \equiv e^{j \frac{2 \pi}{N}}$, the DFT Expression (2.3.2-1) can be expressed as

$$
F_{k}=\sum_{n=0}^{N / 2=1} f_{2 n} e^{j 2 \pi \frac{(2 n) k}{N}}+W_{k} \sum_{n=0}^{N / 2-1} f_{2 n+1} e^{j 2 \pi \frac{(2 n) k}{N}}=F_{k}^{e}+W_{k} F_{k}^{o}
$$


The quantity $F^{e}$ can then be written as the sum of two transforms of length N/4, $F^{e e}$ and $F^{e 0}$ which are formed from the even and odd components as before. This process is continued recursively until the data has been subdivided into Fourier transforms of length one, which is simply the identity operation. In other words, the output of a particular subdivided Fourier transform of length one is related to a particular

input, $F_{k}^{\text {еоееооее..еое }}=f_{n}$. Interestingly, it turns out that the value of $\mathrm{n}$ can be found by setting $\mathrm{e}=0$ and $\mathrm{o}=1$ and bit reversing the pattern of e's and o's [5]. The basic approach of the FFT algorithm is then to first sort the input function into bit reversed order, which gives the results of the length one DFTs. The adjacent pairs of values are then combined until the two halves of the whole data set are combined into the final transform.

More advanced FFT algorithms exist that use special symmetries and subdivide by factors other than two so that there is no restriction on the value of N. It should be noted, however, that the FFT algorithm works most efficiently when the function to be transformed is a power of two. Because of this, it is often advantageous to increase the length of the input function to a power of two by adding zeros to the end of the function, in a process called zero padding.

\subsubsection{Implementation of the Far-Field Transformation}

The plane wave spectrum in expressions (2.2-1) and (2.2-2) represent a twodimensional Fourier transform of the $\mathrm{x}$ and $\mathrm{y}$-components of the measured near-field data. Following the method of [3], the FFT is used to evaluate this integral by first 
approximating it as a discrete process, and then presenting this plane wave spectrum in a form that is suitable for processing with the FFT.

As shown in [3], the plane wave spectrum equations can be approximated as

$$
A\left(k_{x}, k y\right) \approx \frac{\Delta x \Delta y e^{j k_{z} d}}{2 \pi} \sum_{n=0}^{N-1} \sum_{m=0}^{M-1} E(n \Delta x, m \Delta y) e^{j\left(k_{x} n \Delta x+k_{y} m \Delta y\right)} .
$$

We next let

$$
k_{x}=i \Delta k_{x}-\frac{\pi}{\Delta x}, \quad i=0,1,2, \ldots, N-1
$$

and

$$
k_{y}=l \Delta k_{y}-\frac{\pi}{\Delta y}, \quad l=0,1,2, \ldots, M-1
$$

where the values of $\Delta k_{x}$ and $\Delta k_{y}$ are

$$
\Delta k_{x}=\frac{2 \pi}{N \Delta x}
$$

and

$$
\Delta k_{y}=\frac{2 \pi}{M \Delta y} .
$$


Then (2.3.3-1) becomes

$$
\begin{aligned}
& A\left(i \Delta k_{x}-\frac{\pi}{\Delta x}, l \Delta k_{y}-\frac{\pi}{\Delta y}\right)=\frac{\Delta x \Delta y e^{j k_{z} d}}{2 \pi} \sum_{n=0}^{N-1} \sum_{m=0}^{M-1} E(n \Delta x, m \Delta y) e^{j 2 \pi\left(\left(\frac{i n}{N}-\frac{n}{2}\right)+\left(\frac{l m}{M}-\frac{m}{2}\right)\right)} \\
& i=0,1, \ldots, N-1, l=0,1, \ldots, M-1
\end{aligned}
$$

Expression (2.3.3-6) is in a form that is suitable for evaluation using the FFT. In this thesis the data processing will be performed in the MATLAB [6] environment, in which the general two-dimensional FFT is of the form

$$
\begin{aligned}
X(m, n) & =\sum_{p=1}^{M} \sum_{q=1}^{N} x(p, q) e^{-j \frac{2 \pi}{M N}(p-1)(q-1)(m-1)(n-1)} \\
1 & \leq m \leq M, \quad 1 \leq n \leq N
\end{aligned}
$$

and the associated inverse transform (IFFT)

$$
\begin{gathered}
x(p, q)=\frac{1}{M N} \sum_{m=1}^{M} \sum_{n=1}^{N} X(m, n) e^{j \frac{2 \pi}{M N}(p-1)(q-1)(m-1)(n-1)} . \\
1 \leq p \leq M, \quad 1 \leq q \leq N
\end{gathered}
$$

In other words, the x-component of the plane wave spectrum, as in expression (2.3.3-6), can be written in terms of the inverse FFT as 


$$
A_{x}\left(p \Delta k_{x}, q \Delta k_{y}\right)=\frac{\Delta x \Delta y M N}{2 \pi} \operatorname{IFFT2}\left\{E_{x}(m \Delta x, n \Delta y)\right\}
$$

and similarly for the other components, where IFFT2 is the MATLAB two-dimensional inverse FFT function. The FFT algorithm implemented by MATLAB is based on the widely used FFTW, and details of this algorithm can be found in [7].

It should be noted that the result of expressions (2.3.3-6) and (2.3.3-8) will not initially produce the same results. When performing any FFT function in MATLAB, one must use the function FFTSHIFT to obtain the 'expected' result, as achieved by the direct evaluation of expression (2.3.3-6). As mentioned in Section 2.3.1, the DFT (and thus the FFT) produces a periodic output. The 'expected' output of the DFT is centred on the zero frequency component of one period. However, the result of the MATLAB implementation is not centred on the zero-frequency component, but rather starts at the zero-frequency. The MATLAB function FFTSHIFT is used to shift the output so that it is now centred on the zero-frequency component. Furthermore, as mentioned in Section 2.3.2, zero-padding is advantageous as it can improve the efficiency of the FFT algorithms, and although it will not add any new information to the output, the zeropadding will act as a form of interpolation as the output data will have the same size as the zero-padded input. The IFFT2 function in MATLAB allows the user to input values for zero-padding. An example showing the implementation of the far-field transform in MATLAB can be found below in Figure 2.3-1 through Figure 2.3-5. 
Figure 2.3-1 shows a synthetic ${ }^{2} 17 \times 17$ sample point set of near-field data (as would be obtained from a PNF scan) that used a $0.5 \lambda$ spacing between sample points. The associated zero-padded PNF data set is shown in Figure 2.3-2, where the zeros have been added to the end of the data so that it is now a $32 \times 32$ sample point set. Figure $2.3-3$ shows the direct evaluation of expression (2.3.3-6) on the zero-padded data, and is the 'expected' result. Figure 2.3-4 shows the evaluation of expression (2.3.3-6), however this time using IFFT2 function in MATLAB. This does not match the direct implementation of expression (2.3.3-6). Finally, Figure 2.3-5 shows the evaluation of expression (2.3.3-6) using both the IFFT2 and FFTSHIFT functions in MATLAB. The result obtained is identical to that of Figure 2.3-3.

The above mentioned FFT routines are used in the code $A P N F$ that is developed in Chapter 3 to implement the Adaptive Planar Near-Field technique being proposed in this thesis.

\footnotetext{
${ }^{2}$ By this we mean that we simply wrote down the data; it was not measured or obtained from an electromagnetic simulation.
} 


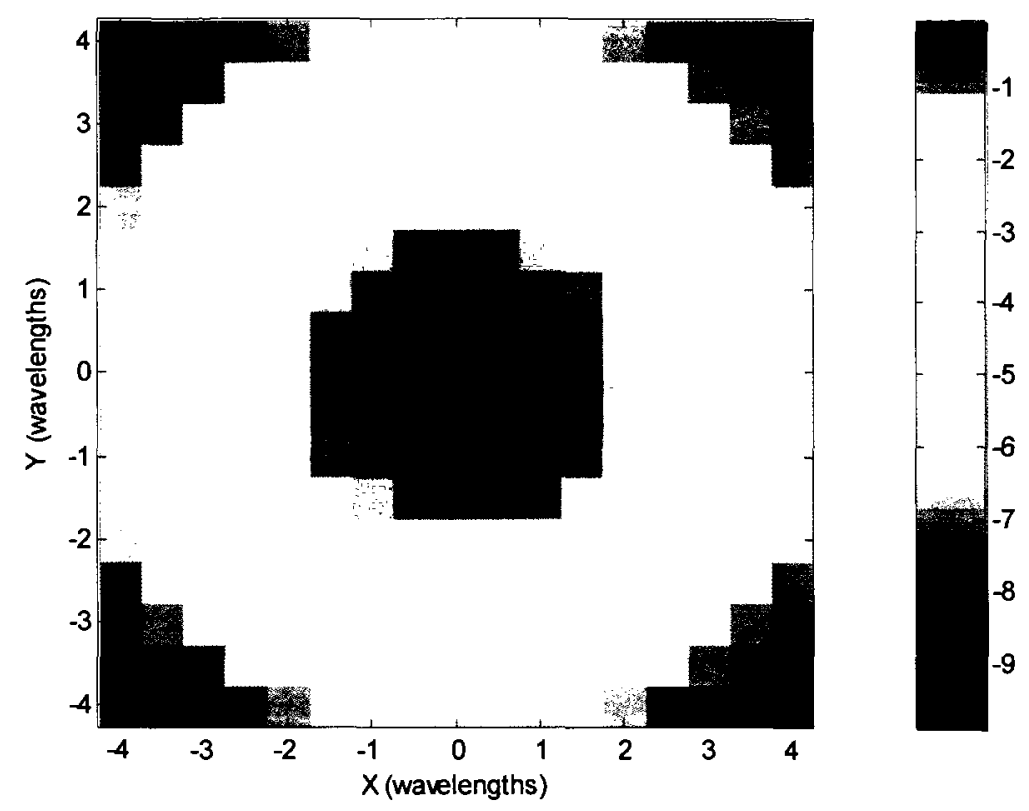

Figure 2.3-1: Simulated $17 \times 17$ PNF data with a 0.5 wavelength spacing, in dB.

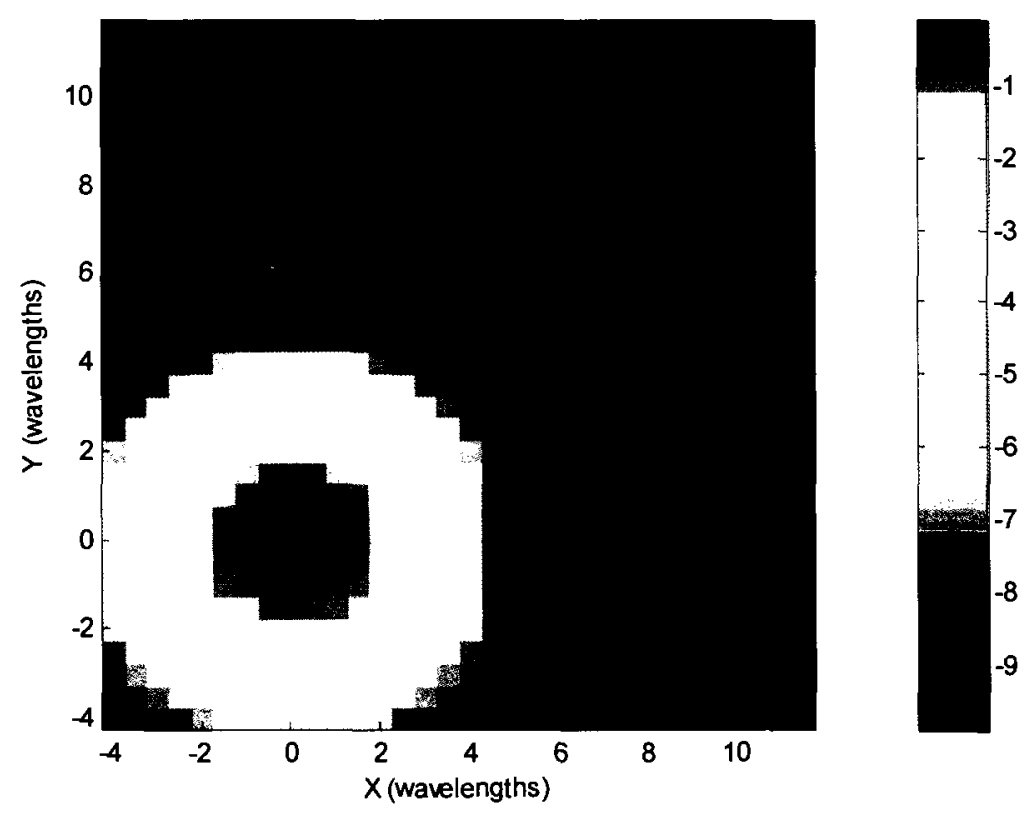

Figure 2.3-2: Simulated 17x17 PNF data zero-padded to 32x32, in dB. 


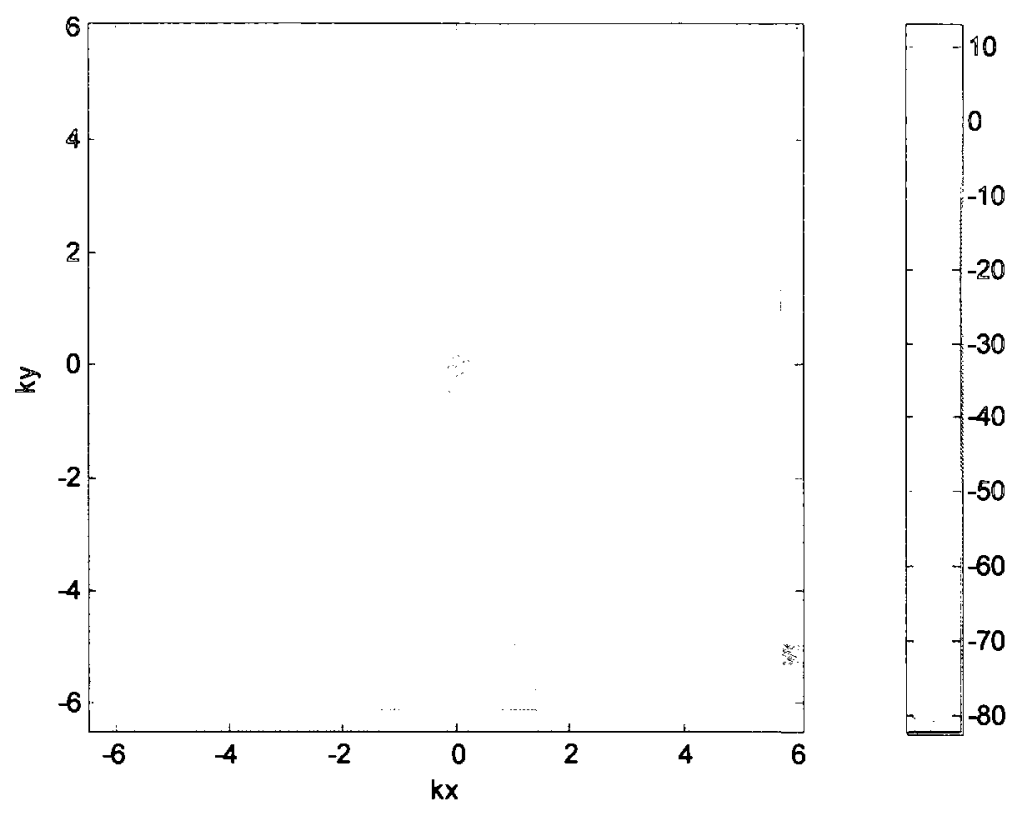

Figure 2.3-3: Far-Field transformation of zero-padded input data generated using a direct evaluation of expression (2.3.3-6), in dB. This is the 'expected' result.

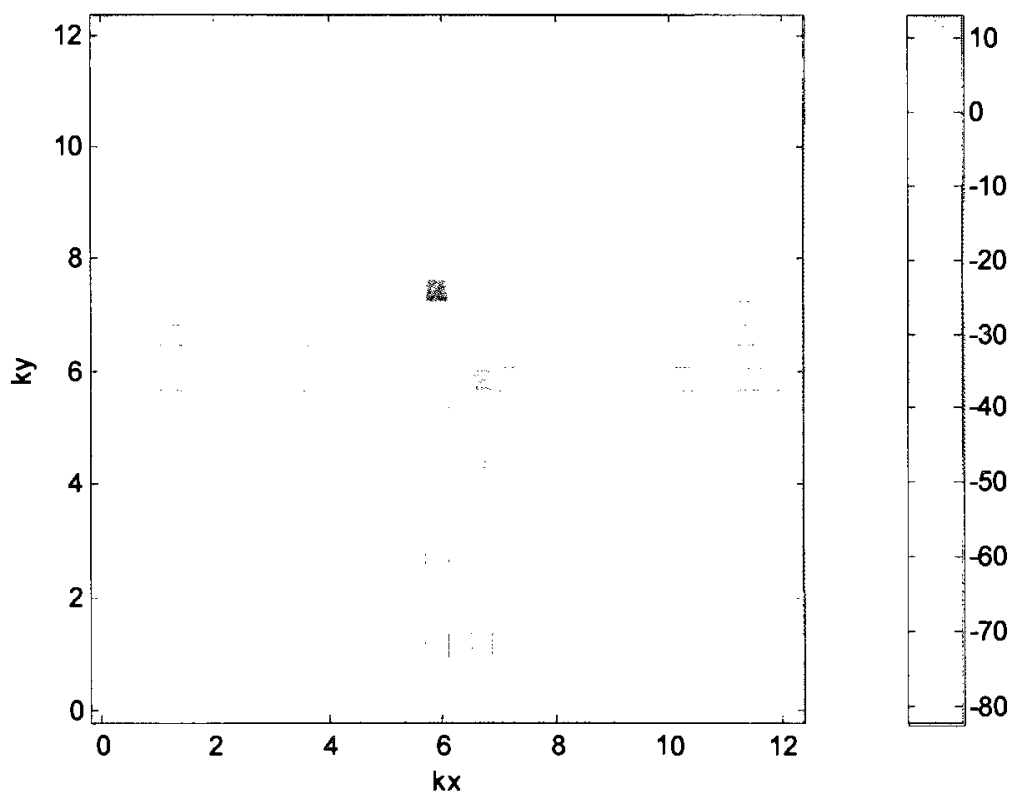

Figure 2.3-4: Far-Field transformation of expression (2.3.3-6) using the IFFT2 function in MATLAB on the zero-padded input data, in dB. 


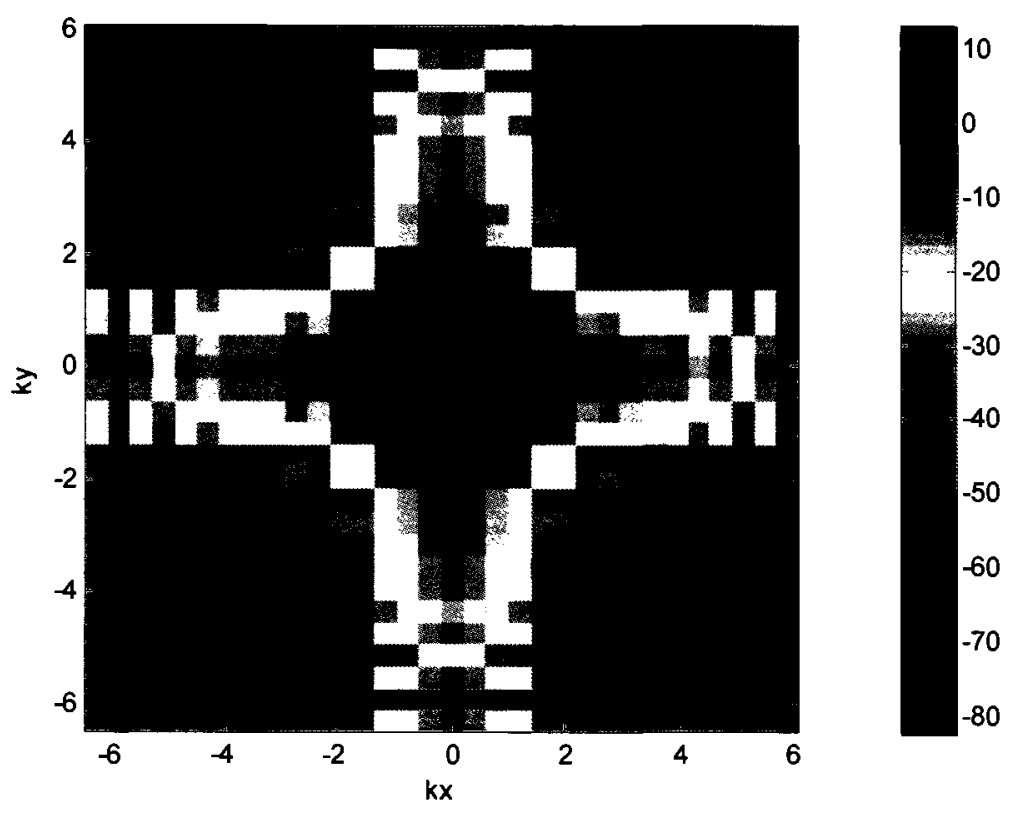

Figure 2.3-5: Far-field transformation of zero-padded input data using the FFTSHIFT and IFFT2 functions in MATLAB to evaluate expression (2.3.3-9), in dB. This now matches the 'expected' result in Figure 2.3-3.

\subsection{Errors in Near- Field Measurements}

There is well-established literature on both the theoretical and practical aspects of PNF measurements [8]-[18], including the theory behind compensation of the effects of the measurement probe, the required spacing between measurement points, the effects of errors in the measurement probe positions, and so forth. It is therefore not necessary to go into much detail here except to mention two types of error to which we will explicitly refer in this thesis, namely truncation error and probe errors.

The electromagnetic theory upon which the PNF technique is based requires the scan area to be an infinite plane. Obviously we are only able to scan over a finite area in practice, the near-field samples beyond this finite area being assumed to be zero; this 
assumption results in so-called truncation error. If you have an antenna of a certain aperture area ${ }^{3}$, and you want to predict the far-zone pattern out to a certain angle off broadside, you need the near-field scan area to be large enough in order to avoid excessive truncation error. In practice this translates to an approximate rule [8], which we will refer to as the rule-of-thumb, given in terms of the minimum linear dimension in some "cut" as

$$
L \geq W+P+2 H \tan \xi
$$

In other words, we have

$$
\xi \leq \tan ^{-1}\left\{\frac{L-W-P}{2 H}\right\} \text {. }
$$

The components of expressions (2.4-1) and (2.4-2) are illustrated in Figure 2.4-1.

If we wish to have a far-zone radiation pattern (after processing of the near-field data acquired over the scan plane) that is valid out to an angle $\xi$ off the broadside direction ${ }^{4}$, then the linear "cut" dimensions just mentioned can be used to determine the size of the scan area. We would like the scan area to be as small as possible in order to reduce the number of data points and hence data acquisition time.

\footnotetext{
${ }^{3}$ PNF testing is not suited to low-directivity (eg. omni-directional or sectoral beam) antennas. The rule-ofthumb is that the AUT directivity needs to be above $15 \mathrm{dBi}$ for PNF testing to be appropriate. In practice, therefore, it is usually reflector antennas, array antennas or horn antennas which are tested in PNF facilities. Thus it is valid to consider the AUT to be an "aperture".

${ }^{4}$ Angle $\xi$ is often referred to as the "angle of validity".
} 


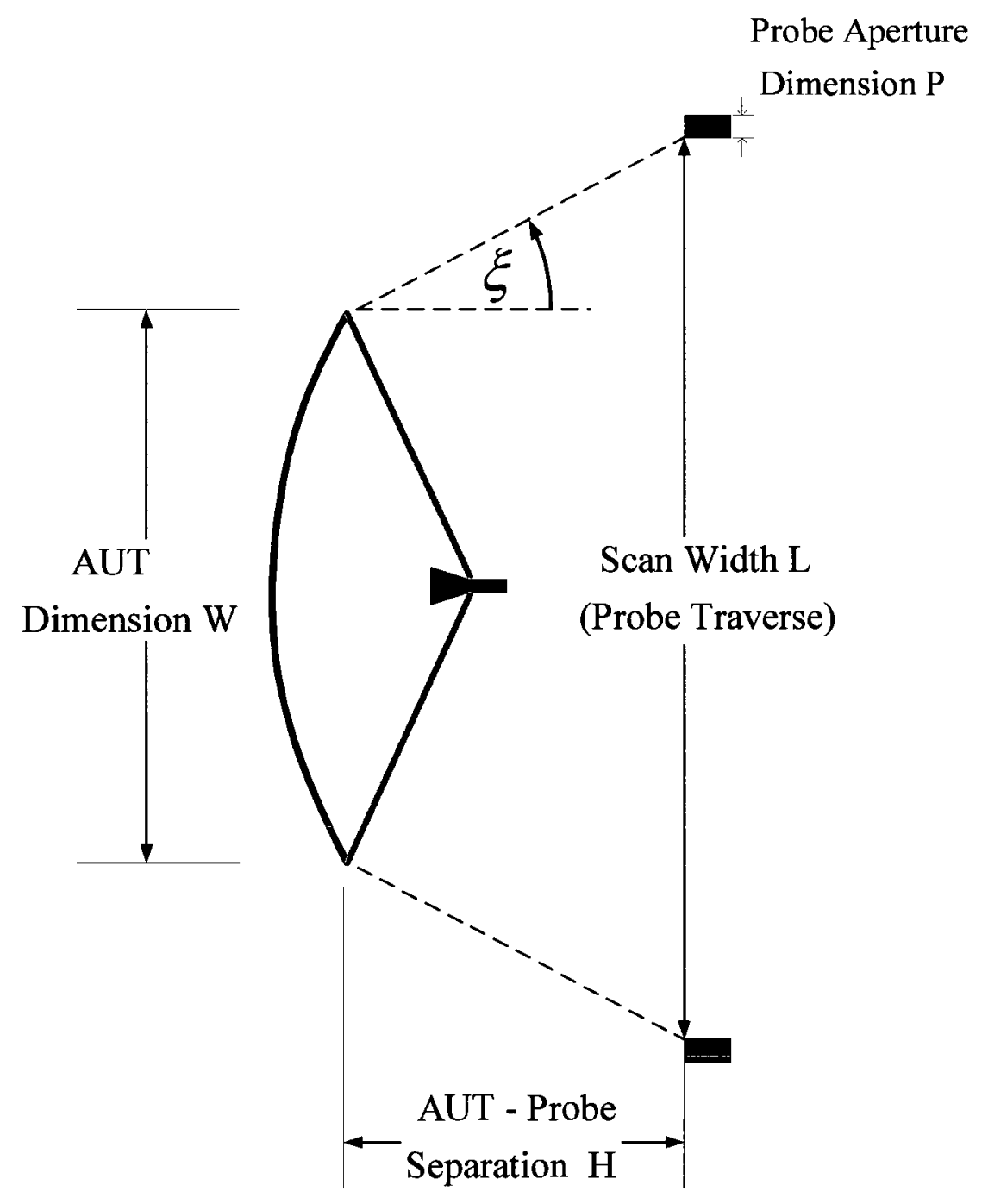

Figure 2.4-1: PNF test set-up schematic

One might immediately think that this limitation could be partially overcome by simply moving the scan plane closer to the physical structure of the AUT. Unfortunately in this case the presence of the probe alters the actual near-field value being sought too drastically, due to interaction between the physical structure of the AUT and the physical structure of the probe. Thus we must increase the value of the separation $\mathrm{H}$ until this is 
not the case $e^{5}$. This unwanted "mutual coupling" between the probe and AUT can be further decreased by one of a number of additional methods:

- The use of absorber material around the probe.

- Thinning of the edge of the probe to decrease its reflective area.

- Use of an isolator at the probe input/output port.

The probe does not measure the field at a point (since it is of finite size and not infinitesimal), and has a broad radiation pattern of its own but is not isotropic. This must be compensated for in order to improve the measurement accuracy. Such probe pattern compensation is conveniently incorporated into the PNF processing algorithm as a deconvolution [1], [4], [8].

\subsection{Enhancements to Planar Near-Field Measurement Techniques}

\subsubsection{Overview}

The possibility of using a reduced amount of PNF data and hence reducing the test times has been considered by others using two approaches:

- Near-field data re-computation over a plane closer to the AUT. This will be discussed in Section 2.5.2.

\footnotetext{
${ }^{5}$ This is often stated as reducing the mutual coupling between the AUT and the probe. While not an incorrect statement, it can be misleading. If there were no mutual coupling between the AUT and the probe we would not be able to measure the near-fields of the AUT. We want to maintain such mutual coupling, but without the interaction between the AUT and the probe altering the radiation characteristics of either of these antennas. It is the later effect that is meant in near-field measurement parlance by the term "mutual coupling between the AUT and probe".
} 
- Near-field data selection, as will be described in Section 2.5.3.

If one has a PNF scanner of a fixed size (in other words, is able to achieve a certain scan area), and the far-zone pattern is required out to a certain angle off the boresight axis, then the physical size of the AUT will be limited. If we wish to test a larger AUT using the conventional PNF processing discussed up to now (to find the farzone radiation pattern out to the same angles), then a larger PNF scanner would be required to obtain data points over a larger scan area. Several authors have addressed the issue of trying to circumvent this restriction in some way (without of course incurring errors), thereby allowing a slightly larger AUT to be tested using a given PNF scanner ${ }^{6}$. These enhancements can be can be broadly grouped as follows:

- Near-field data extrapolation over the scan plane, which is the topic of Section 2.5.4.

- Near-field data reconstruction over the scan plane, as reviewed in Section 2.5.5.

- The scan plane decomposition mentioned in Section 2.5.6.

The latter methods are not strictly speaking PNF methods, since they require data to be acquired on more than one plane, and so will likely not reduce test times. They will nevertheless be mentioned here.

\footnotetext{
${ }^{6}$ This would translate to test time reductions too, in that one would require fewer data points (since one is using a smaller scan area) than would be required for the conventional PNF method for the same AUT size.
} 


\subsubsection{Near-Field Data Re-Computation Over a Plane Closer to the AUT}

The surface equivalence principle states the following: If we have the tangential electric field $\hat{n} \times \bar{E}$ and tangential magnetic field $\hat{n} \times \bar{H}$ on some arbitrary closed surface $S_{e q}$, where $\hat{n}$ is the unit normal at each point on the surface, then these can be used to determine equivalent surface current densities $\bar{M}_{s}=-\hat{n} \times \bar{E}$ and $\bar{J}_{s}=\hat{n} \times \bar{H}$ from which (via the usual radiation integrals) the electromagnetic fields can be determined at any other point off the surface, and in particular in the far-zone. If the surface $S_{e q}$ is selected to be an infinitely large planar surface, then image theory can be used to show that the fields at any point can be found in terms of the magnetic surface current density $\bar{M}_{s}=-2 \hat{n} \times \bar{E}$ only, and for observation points in the far-zone the radiation integrals take the form of a two-dimensional Fourier transform of the $\bar{M}_{s}=-2 \hat{n} \times \bar{E}$. We can interpret the PNF measurement process in terms of this approach.

The surface $S_{e q}$ corresponds to the scan plane. Data acquisition supplies the values of the field $\hat{n} \times \bar{E}$ at all points over the scan area, and the fields in the far-zone are then the Fourier transform of the equivalent source $\bar{M}_{s}=-2 \hat{n} \times \bar{E}$. The scan plane can be viewed as a source plane ${ }^{7}$ where the equivalent currents used to find the fields are located. The final expressions would be identical to (2.2-14) through (2.2-16). However, we can use $\bar{M}_{s}=-2 \hat{n} \times \bar{E}$ to find the fields at any point, and not just in the far-zone; if the observation point is not in the far-zone the radiation integral just does not reduce to a Fourier transform.

\footnotetext{
${ }^{7}$ The distance between the AUT and the source plane determines the angle of validity, as discussed in Section 2.4.
} 
Suppose we have acquired the fields $\hat{n} \times \bar{E}$ over the scan plane and wish to use the plane wave spectrum approach ${ }^{8}$ in Section 2.2 to find the fields over some plane closer to the $\mathrm{AUT}^{9}$. One first finds the plane wave spectrum by taking the Fourier transform of the acquired near-field data, then multiplies the plane wave spectrum by the factor $e^{j k_{z} \Delta z}=e^{j \sqrt{k^{2}-k_{x}^{2}-k_{y}^{2}}}$, and then takes the inverse Fourier transform of the shifted plane wave spectrum. This gives the fields over a plane a distance $\Delta z$ behind the scan plane.

An alternative method for such back-projection is the integral equation approach. One begins with an integral expression ${ }^{10}$ for the field $\bar{E}$ at any observation point in space due to an unknown magnetic current density $\bar{M}_{s}$ over a plane $\mathrm{z}=0$. An integral equation for the unknown $\bar{M}_{s}$ is obtained by enforcing the fact that at points on the plane $\mathrm{z}=\mathrm{d}$ we know from measurements what $\hat{n} \times \bar{E}$ is. The method of moments is then used to solve for the $\bar{M}_{s}$ over the plane $\mathrm{z}=0$. The equivalent source $\bar{M}_{s}$ is closer to the actual AUT than the PNF scan plane, and so the far-zone results obtained by Fourier transforming this source distribution should have a larger angle of validity than if we used the source distribution directly on the scan plane. The shape and location of $\bar{M}_{s}$ can be determined based on the geometry of the AUT. This integral equation approach is the subject of references [19] through [43], where many topics are described. Some of the references do not all deal with the PNF case but instead one of the following:

\footnotetext{
${ }^{8}$ We will also refer to this as the direct Fourier transform approach.

${ }^{9}$ This is process called back-projection (or holographic projection, although this may be a misnomer) in the near-field antenna measurement literature.

${ }^{10}$ The observation point is not in the far-zone of the current density and so the integral expression in question is not simply a Fourier transform.
} 
- They consider taking near-field data acquired over an arbitrary surface and find the equivalent currents over a plane inside that surface.

- They consider taking data acquired over a spherical surface and find equivalent currents that allow one to find the fields inside even the minimum sphere enclosing the AUT.

Those that do consider the PNF case discuss ways of accelerating the moment method solution of the integral equation used to find the equivalent currents. It is stated in [22], [24] that (in the PNF context) the truncation error in the integral equation approach is less than that using the plane wave spectrum one, and so the angle of validity is indeed larger for the same size scan area ${ }^{11}$. However, we do not consider this fact to have been demonstrated unequivocally. For instance, the authors either use horn antennas (which do not have particularly low sidelobes) or show rather coarsely plotted patterns (with large angular steps resulting in jagged looking patterns) that leave one unconvinced. In another case the far-zone pattern obtained using a specific scan area and the direct Fourier transform approach is compared to that obtained with the integral equation approach (and equivalent currents over a plane closer to the AUT) using a much smaller scan area. The radiation pattern results from the two cases are shown to agree out to $40^{\circ}$ from the

\footnotetext{
${ }^{11}$ Interestingly, in [24] it is shown that if the scan areas are infinite in size the direct Fourier transform approach and the integral equation approach are mathematically identical. It is furthermore explained there that "the integral equation method is not creating information that is not there in the measured data. The integral equation method has less truncation error" since it is in essence using a model-based-parameterestimation technique and so "will always provide higher resolution than the conventional" the Fourier transform back-projection procedure. Perhaps a more fundamental explanation is that provided by the authors of [45]. They explain that when we use of the direct Fourier transform (plane wave spectrum) approach on the near-field data information is lost since only the visible part of the spectrum - those points of the spectrum $\left(k_{x}, k_{y}\right)$ inside the circle of radius $k$ - is available, which implies a resolution of one wavelength in the reconstructed currents.
} 
broadside direction. Unfortunately they do not show how well the direct Fourier transform approach performs using the smaller near-field data set. It is claimed that the integral equation approach has the potential to use a near-field data set that contains only $25 \%$ of the data points used in the Fourier transform approach for the same angle of validity. However, even if this were to be shown (in a more robust way using many different AUT types), the processing time for each frequency is very much larger than that of the direct Fourier transform approach ${ }^{12}$. What one gains in data acquisition time one loses in processing time. One can argue that an AUT can be tested and then removed while its data is being processed, so freeing up the PNF range for the next AUT. In practice it is more likely that engineers would want to see what the performance is before dismounting it; this is especially true when the PNF range is being used as a diagnostic tool or an antenna is being calibrated or fine-tuned in some way.

\subsubsection{Near-Field Data Selection over the Scan Plane}

In [44], the authors "investigate alternative sampling techniques that reduce the time required to collect the near-field data". This is perhaps the first published work that is explicitly aimed at test time reduction. The technique, "which reduces the number of sample points by $50 \%$, makes use of the derivative theorem in Fourier transform theory" [44]. While conventional sampling theory requires one to sample the near-field data points at twice the highest spatial frequency, there is a more general sampling theorem that requires one to sample at only the highest spatial frequency of not only the near-field

\footnotetext{
${ }^{12}$ One is solving a method of moments problem with unknowns on a surface that is larger than that of the AUT. If the AUT is an electrically large reflector, for instance, this could be prohibitive. The computation time could be hours, and might require special computation resources.
} 
values but also their spatial derivatives. There are a number of drawbacks with this approach. Firstly, the near-to-far-field transformation is more complicated, and a probecompensation technique does not appear to have been devised for this approach. Secondly, and more importantly, suitable probes have not been developed for sampling the field derivatives. This might be the reason that, even though the above data reduction approach was suggested more than 20 years ago, it has never been taken up by the antenna measurement community.

In Section 2.1 we noted that we implicitly assume that the near-field values on the scan plane are zero outside the scan area. Now there is no theoretical requirement that the scan area be rectangular in shape. In [45], in order to reduce the number of data points required, the author ignores all near-field data points below a certain amplitude, thereby forming a scan area whose boundary is of an irregular shape. This approach requires an initial exploratory run over a complete large scan area whose size is determined using guidelines such as that given in Section 2.4, providing data such as that in the top image of Figure 2.4-1 for example. This is used to examine the near-field magnitude contours, and those data points whose near-field magnitude values are below some selected threshold value are "blanked out", as indicated in the bottom image of Figure 2.4-1, and the near-fields at these locations are not sampled (but assumed to be zero) in any subsequent near-field scans. In other words, in any following scans, the probe locus is such that the probe does not even move to these "blanked out" points. If this is done testtime reductions of $10 \%-20 \%$ are possible [45]. The disadvantage of this approach is that, at least until the particular class of AUT is very well understood, one will always wonder if a particular AUT being tested might be such that the near-field magnitude contours are 
perhaps slightly different from those observed in the exploratory run mentioned. This could occur if the testing is being done for diagnostic purposes and some adjustments have been made to the AUT.
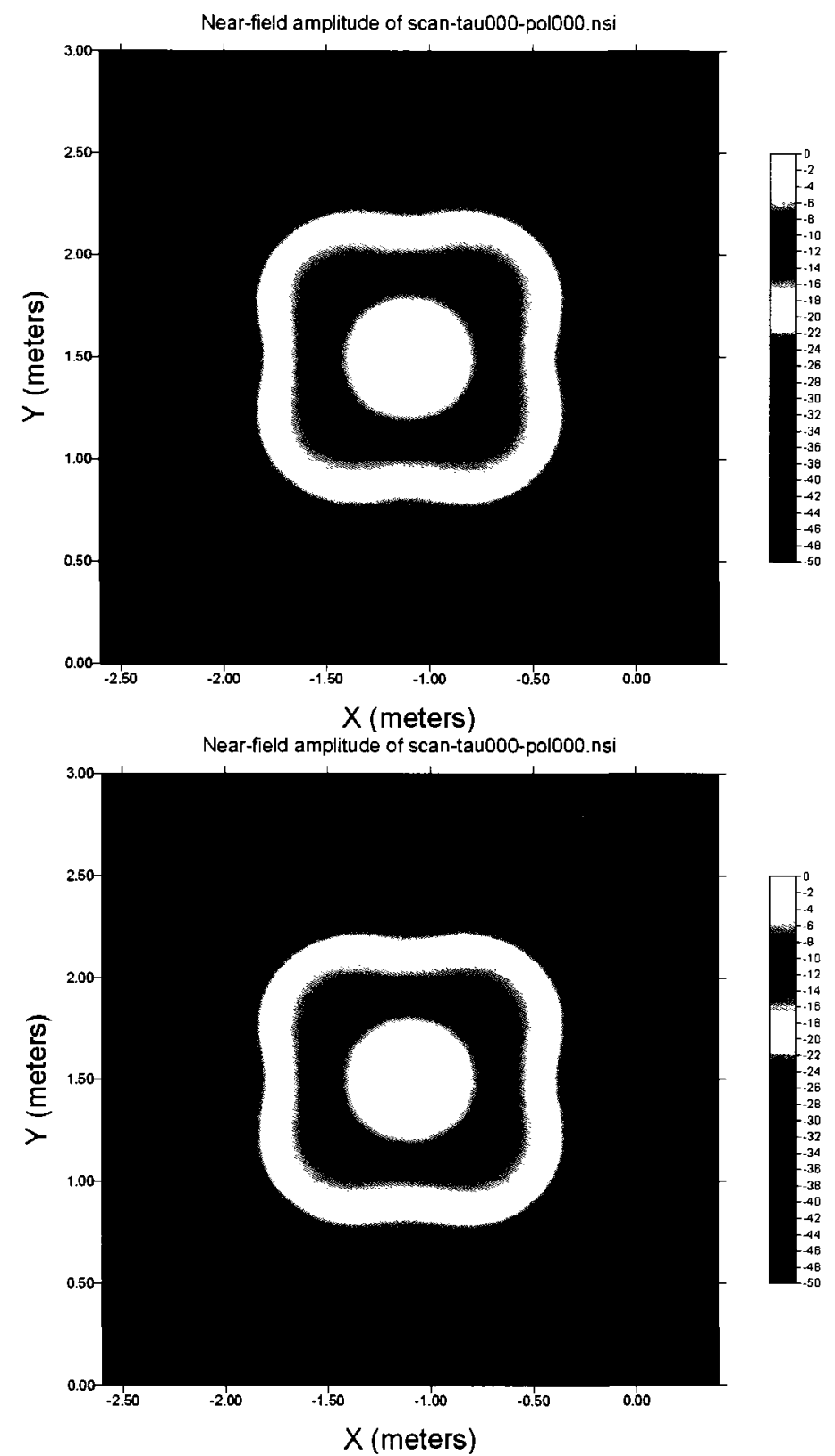

Figure 2.4-1: Initial large scan area (top); Scan area with data points (shown blackened out) whose near-field amplitude are below a selected threshold value ignored (bottom). (After [45]) 


\subsubsection{Near-Field Data Extrapolation over the Scan Plane}

In this approach near-field data is acquired over the scan area. Near-field data outside of the scan area (but at points on the scan plane) is estimated using a complicated extrapolation algorithm on the available data that takes advantage of information on the AUT size [46], [47]. This means one effectively has near-field data over a larger scan area than was actually measured. This near-field data can then be used in the conventional way to determine the far-zone fields. Because the near-field data occupies a larger scan area the angle of validity is larger ${ }^{13}$. This in turn means that for a specified angle of validity one can acquire data points over a smaller scan area that would normally be required and determine the remaining data points through extrapolation. This would potentially lead to test time reduction. Unfortunately, in spite of the use of sophisticated regularisation procedures to overcome the ill-conditioned nature of the process, "only a few samples outside of the measurement zone can be reliably recovered" [47].

\subsubsection{Near-Field Data Reconstruction over the Scan Plane}

In order to overcome the limitations of the technique in Section 2.5.4, Bucci et al. [48], [49] came up with a different idea. It uses the so-called sampling representation [50] that exploits known geometrical information about the AUT (namely its size, and thus the spatial bandwidth of its fields). It is not a mere extrapolation procedure, but uses the sampling theory to determine "how the information content of the field radiated by the AUT is distributed in the space around the AUT" [49]; hence the term "reconstruction". It

\footnotetext{
${ }^{13}$ Alternatively, we can say that the truncation error is reduced.
} 
"recovers" the near-field values over the scan plane beyond the scan area accessible by the PNF scanner, and is capable of eliminating truncation error. Unfortunately the information needed to do so must be obtained by acquiring data through movement of the probe not only over the scan area on the conventional scan plane but also perpendicular to it. Thus while the method is effective, is not computationally taxing, and could extend the applicability of a PNF scanner of limited size (in the xy-plane) as long as it can move along the z-axis, it does not lead to test time reduction.

\subsubsection{Plane Wave Spectrum Extrapolation}

Instead of extrapolating the near-field data over the scan plane and then using it to find the far-zone fields, the technique described in [51], [52], [53] extends the plane wave spectrum itself. The ill-conditioning of numerical processes that perform such tasks (and hence their susceptibility to noise) is circumvented by extending the spectrum subject to a constraint that the equivalent source is concentrated in the AUT aperture ${ }^{14}$. The particular numerical technique uses an iterative technique called the method of alternating projections, in particular a specific case thereof called the Gerchberg-Papoulis algorithm. It starts by taking the original acquired data and finding the plane wave spectrum. This plane wave spectrum has a limited "reliable region" (within the angles of validity). This is then back-projected (inverse Fourier transform) to the AUT aperture plane. All points on the aperture plane that lie outside the AUT aperture are set to zero, and the plane wave spectrum is then found from this constrained aperture distribution. This plane wave spectrum will have points outside the reliable region. These are retained. However, the

\footnotetext{
${ }^{14}$ Once again, known information on the AUT is being used to advantage.
} 
plane wave spectrum samples that lie in the reliable region are replaced by the starting values. These are then projected back to the aperture plane and the steps mentioned earlier repeated iteratively, until some convergence criteria are met. Examples of such criteria are given in [51], [52], [53]. Although not mentioned by the authors, it is known that the method of projections (no matter what it is used for) is susceptible to so-called "traps and tunnels", and may not converge, [54], [55]. This would not be acceptable in an antenna measurement environment. Further testing of the method in [51], [52], [53] might reveal that this is not the case for this specific application, but with the limited two cases checked (one synthetic, the other experimental data) one cannot be certain of this. If indeed so, this could be an effective method for test time reduction, especially since the computing time is much less than that of the integral equation methods described in Section 2.5.2.

\subsection{Concluding Remarks}

It is the data acquisition time (that is, the actual physical movement of the probe) that dominates testing time, since it is measured in minutes and hours, whereas data processing time (at least when using the FFT approach to obtain the far-zone fields from the scan plane data) is measured in seconds. This was not necessarily the case in the earlier days of PNF testing, but is certainly so now.

Although not the major contribution of this thesis, the implementation details presented in Section 2.3 are not readily available elsewhere in their entirety, and so it was useful to provide them here. Section 2.4 points the reader to the important references that have examined errors in PNF measurements, and an important rule-of-thumb that relates 
the angular range over which a reliable far-zone pattern can be obtained to the available scanning area, the AUT size and the distance between the probe and AUT.

Section 2.5 gives a review of work that has been done to reduce the truncation error associated with the given finite scan area of a PNF system. The idea in the reviewed work is that if the truncation error can be reduced for a given scan size then it is possible to obtain far-zone patterns out to a larger angle off broadside than would normally be possible using the conventional approach of Section 2.2. This might at first glance seem to be the obvious way to go to reduce test times, and yet there does not seem to have been a rush to implementing any of it. This is due to one or more of the following reasons:

a) The additional computation that is needed becomes prohibitive (even by present standards) when the AUT is electrically large, which is precisely the regime where PNF testing is most widely used.

b) The computational problem is beset with ill-conditioning problems. This would be unacceptable in an antenna test environment.

c) Additional data points are required that do not lie on the PNF measurement plane (or on a cylinder or sphere) but on planes orthogonal to the measurement plane. This is either inconvenient or simply not possible with standard PNF systems, and anyway does not reduce test times.

d) The computations required might be susceptible to so-called "traps and tunnels" and may not converge.

In Chapter 3 we therefore propose a test time reduction approach that builds feedback (that is, adaptivity) into near-field measurements. 


\subsection{References for Chapter 2}

[1] D.T.Paris, W.M.Leach Jr., \& E.B.Joy, "Basic theory of probe-compensated near-field measurements", IEEE Transactions on Antennas and Propagation, vol. AP-26, no. 3, pp. 373-379, May 1978.

[2] J.J.H.Wang, "An examination of the theory and practices of planar near-field measurement", IEEE Transactions on Antennas \& Propagation, vol. 36, no. 6, pp. 746-753, Jun. 1988.

[3] Y.Rahmat-Samii, L.I.Williams, \& R.G.Yaccarino, "The UCLA bi-polar planar-nearfield antenna-measurement and diagnostics range", IEEE Antennas and Propagation Magazine, vol. 37, no. 6, pp. 16-35, Dec. 1995.

[4] A.D.Yaghjian, "An overview of near-field antenna measurements", IEEE Transactions on Antennas \& Propagation, vol. 34, no. 1, pp. 30-45, Jan. 1986.

[5] W.H.Press, S.A.Teukolsky, W.T.Vetterling, and B.P.Flannery, Numerical Recipes in $C$ : The Art of Scientific Computing, $2^{\text {nd }}$ ed. Cambridge: Cambridge University Press, 1992.

[6] $M A T L A B^{T M}$ is a trademark of The MathWorks Inc., USA.

[7] M. Frigo \& S.G. Johnson, "The design and implementation of FFTW3", Proceedings of the IEEE, vol. 93, no. 2, pp. 216-231, Feb. 2005.

[8] A.C.Newell, "Error analysis techniques for planar near-field measurements", IEEE Transactions on Antennas \& Propagation, vol. 36, no. 6, pp. 754-768, Jun. 1988.

[9] S.F. Gregson, J. McCormic, \& C. Parini, Principles of Planar Near-Field Antenna Measurements. London, UK: The Institution of Engineering and Technology, 2007

[10] E.B.Joy \& D.T.Paris, "Spatial sampling and filtering in near-field measurements", IEEE Transactions on Antennas and Propagation, vol. AP-20, no. 3, pp. 253-261, May 1972.

[11] N.Mao, X.Zhang, \& Y.Li, "Investigation of sampling spacing in planar near-field antenna measurements", Proceedings of the Asia-Pacific Microwave Conference, pp. 902-905, 2001.

[12] E.B.Joy, W.M.Leach,Jr., G.P.Rodrigue, \& D.T.Paris, “Applications of probecompensated near-field measurements", IEEE Transactions on Antennas and Propagation, vol. AP-26, no. 3, pp. 379-389, May 1978. 
[13] A.C.Newell, R.D.Ward, \& E.J.McFarlane, "Gain and power parameter measurements using planar near-field techniques", IEEE Transactions on Antennas \& Propagation, vol. 36, no. 6, pp. 792-803, Jun. 1988.

[14] E.B.Joy, "Near-field range qualification methodology", IEEE Transactions on Antennas \& Propagation, vol. 36, no. 6, pp. 836-844, Jun. 1988.

[15] A.G.Repjar, A.C.Newell, \& M.H.Francis, "Accurate determination of planar nearfield correction parameters for linearly polarized probes", IEEE Transactions on Antennas \& Propagation, vol. 36, no. 6, pp. 855-868, Jun. 1988.

[16] L.A.Muth \& R.L.Lewis, "A general technique to correct probe position errors in planar near-field measurements to arbitrary accuracy", IEEE Transactions on Antennas \& Propagation, vol. 38, no. 12, pp. 1925-1932, Dec. 1990.

[17] O.M.Bucci,G.Schirinzi, \& G.Leone, "A compensation technique for positioning errors in planar near-field measurements", IEEE Transactions on Antennas \& Propagation, vol. 36, no. 8, pp. 1167-1172, Aug. 1988.

[18] D.J.Janse van Rensburg, "A technique to evaluate the impact of flex cable phase instability on mm-wave planar near-field measurement accuracies", Proc. 1999 ESTEC Antenna Conference, The Netherlands, May 1999.

[19] J.Säily, P.Eskelinen, \& A.V.Räisänen, "Pilot signal-based real-time measurement and correction of phase errors caused by microwave cable flexing in planar nearfield tests", IEEE Transactions on Antennas and Propagation, vol. 51, no. 2, pp. 195-200, Feb. 2003.

[20] S.Ponnapalli, "Near-field to far-field transformation utilizing the conjugate gradient method", Progress in Electromagnetics Research (PIER), vol. 5, pp. 391-422, 1991.

[21] S.Ponnapalli, T.K.Sarkar, \& P.Petre, "Near-field to far-field transformation using an equivalent current approach", IEEE Int. AP-S Symposium Digest, pp. 1458-1461, 1991.

[22] P.Petre, T.K.Sarkar, S.Ponnapalli, \& B.Szekeres, "Integral equation solution for near-field to far-field transformation", Proc. Int. Conf. Computation in Electromagnetics, pp. 218-221, Nov. 1991.

[23] P.Petre \& T.K.Sarkar, "Planar near-field to far-field transformation using an equivalent magnetic current approach", IEEE Transactions on Antennas and Propagation, vol. 40, no. 11, pp. 1348-1356, Nov. 1992.

[24] P.Petre \& T.K.Sarkar, "Planar near-field to far-field transformation using an array of dipole probes", IEEE Transactions on Antennas and Propagation, vol. 42, no. 4, pp. 534-537, Apr. 1994. 
[25] P.Petre \& T.K.Sarkar, "Differences between modal expansion and integral equation methods for planar near-field to far-field transformation", Progress in Electromagnetics Research, vol.12, pp. 37-56, 1996.

[26] A.Taaghol \& T.K.Sarkar, "Near-field to near/far-field transformation for arbitrary near-field geometry utilizing an equivalent magnetic current", IEEE Transactions on Electromagnetic Compatibility, vol. 38, no. 3, pp. 536-542, Aug. 1996.

[27] T.K.Sarkar \& A.Taaghol, "Near-field to near/far-field transformation for arbitrary near-field geometry utilizing an equivalent magnetic current and MoM", IEEE Transactions on Antennas and Propagation, vol. 47, no. 3, pp. 566-573, Mar. 1999.

[28] J.R.Pérez \& J.Basterrechea, "Antenna far-fields from planar acquisition using microgenetic algorithms", IEE Electronic Letters, vol. 39, no. 13, pp. 948-949, Jun. 2003.

[29] J.R. Pérez \& J. Basterrechea, "GA based prediction of antenna radiation patterns from planar near-field samples", Microwave and Optical Technology Letters, vol. 37, pp. 235-236, 2003.

[30] J.R. Pérez \& J. Basterrechea, "Analysis of different GA strategies applied to antenna far-fields reconstruction from planar acquisition", Microwave and Optical Technology Letters, vol. 39, pp. 422-426, 2003.

[31] J.R. Pérez and J. Basterrechea, "Antenna far-field pattern reconstruction using equivalent currents and genetic algorithms", Microwave and Optical Technology Letters, vol.42, pp. 21-25, 2004.

[32] J.R. Pérez \& J. Basterrechea, "Particle-swarm optimization and its application to antenna far-field pattern prediction from planar scanning", Microwave and Optical Technology Letters, vol. 44, pp. 398-403, 2005.

[33] J.R. Pérez and J. Basterrechea, "Comparison of different heuristic optimization methods for near-field antenna measurements", IEEE Transactions on Antennas Propagation, vol. 55, pp. 549-555, 2007.

[34] J. R. Pérez \& J.Basterrechea, "Particle swarms applied to array synthesis and planar near-field antenna measurements", Microwave and Optical Technology Letters, vol. 50 , no. 2, pp. 544-548, Feb.2008.

[35] F.Las-Heras, B.Galocha, \& J.L.Besada, "Equivalent source modeling and reconstruction for antenna measurement and synthesis", IEEE AP-S Int. Symp. Digest, vol. 1, pp. 156-159, 1997.

[36] F.Las-Heras, "Sequential reconstruction of equivalent currents from spherical acquisition", IEEE Electronics Letters, vol. 35, no. 3, pp. 211-212, Feb.1999. 
[37] F.Las-Heras \& B.Galocha, "Planar source reconstruction and far field calculation from near field hemispherical acquisition", Proc. 21st Annual Meeting \& Symp. of the Antenna Measurement Techniques Association (AMTA), pp. 17-21, 1999.

[38] F. Las-Heras, B. Galocha, \& J. L. Besada, "Far-field performance of linear Antennas determined from near-field data," IEEE Transactions on Antennas and Propagation, pp. 408-410, Mar. 2002.

[39] Y.Alvarez, F.Las-Heras, \& M.R.Pino, "Integral equation algorithms for equivalent currents distribution retrieval over arbitrary three-dimensional surfaces", Proc. EuCAP 2006, Nov. 2006.

[40] F.Las-Heras, M.R.Pino, S.Loredo, Y.Alvarez, \& T.K.Sarkar, "Evaluating near-field radiation patterns of commercial antennas", IEEE Transactions on Antennas and Propagation, vol. 54, no. 8, pp. 2198-2207, Aug. 2006.

[41] Y.Alvarez, F.Las-Heras, M.R.Pino, \& M.H.Alvarez, "Sources rreconstruction techniques for the diagnosis and characterization of antennas of complex geometry," Proc. 3rd International Conference on Near-field Imaging and Characterization (ICONIC), pp. 182-187, Jun. 2007.

[42] Y.Álvarez, F.Las-Heras, \& M.R.Pino, "Reconstruction of equivalent currents eistribution over arbitrary three-dimensional surfaces based on integral equation algorithms", IEEE Transactions on Antennas and Propagation, vol. 55, no. 12, pp. 3460-3468, Dec. 2007.

[43] Y.Álvarez, F.Las-Heras, \& M.R.Pino, "On the comparison between the spherical wave expansion and the sources reconstruction method", IEEE Transactions on Antennas and Propagation, vol. 56, no. 10, pp. 3337-3341, Oct. 2008.

[44] L.E.Corey \& D.R.O'Neil, "Differential sampling of planar near-fields", IEEE AP-S Symp. Digest, pp.191-194, June 1986.

[45] D.J.Janse van Rensburg, "Scan-plane reduction techniques for planar near-field antenna measurements", IEEE Antennas and Propagation Magazine, vol. 46, no. 6, pp. 179-184, Dec. 2004.

[46] O.M.Bucci, G.D'Elia, \& M.D.Migliore, "A new strategy to reduce the truncation error in near-field/far-field transformations", Radio Science, vol. 35, no. 1, pp. 3-18, Jan.-Feb. 2000.

[47] J-C.Bolomey, O.M.Bucci, L.Casavola, G.D'Elia, M.D.Migliore, \& A.Zivyat, "Reduction of truncation error in near-field measurements of antennas of basestation mobile communication systems", IEEE Transactions on Antennas \& Propagation, vol. 52, no. 2, pp. 593-602, Feb. 2004. 
[48] O.M.Bucci \& M.D.Migliore, "A new method for avoiding the truncation error in near-field antennas measurements", IEEE Transactions on Antennas \& Propagation, vol. 54, no. 10, pp. 2940-2952, Oct. 2006.

[49] O.M.Bucci \& M.D.Migliore, "Strategy to avoid truncation error in planar and cylindrical near-field antenna measurement setups", IEE Electronic Letters, vol. 39, no. 10, pp. 765-766, May 2003.

[50] O.M.Bucci, C.Gennarelli, \& C.Savaresse, "Representation of electromagnetic fields over arbitrary surfaces by a finite and nonredundant number of samples", IEEE Transactions on Antennas \& Propagation, vol. 46, pp. 351-359, 1998.

[51] E.Martini, O.Breinbjerg \& S.Maci, "Reduction of truncation errors in planar nearfield aperture antenna measurements using the method of alternating orthogonal projections", Proc. First European Conf. Antennas \& Propagation (EuCAP 2006), Nov. 2006.

[52] E.Martini, O.Breinbjerg, \& S.Maci, "A convergence criterion for the iterative Gerchberg-Papoulis algorithm applied to truncation error reduction in planar nearfield measurements", Proc. Second European Conf. Antennas \& Propagation (EuCAP 2007), Nov.2007.

[53] E.Martini, O.Breinbjerg, \& S.Maci, "Reduction of truncation errors in planar nearfield aperture antenna measurements using the Gerchberg-Papoulis algorithm", IEEE Transactions on Antennas \& Propagation, vol. 56, no. 11, pp. 3485-3493, Nov. 2008.

[54] H.Stark \& Y.Yang, Vector Space Projections: A Numerical Approach To Signal And Image Processing, Neural Nets, And Optics, New York: Wiley, 1998

[55] E. Botha, J. Joubert, D. A. McNamara, "Alternative formulation and applications aspects of the generalized projection method for array antenna synthesis", Int. J. Microwave Millimeter-Wave Computer-Aided Engineering, Jul. 2009. 


\section{CHAPTER 3 - THE ADAPTIVE ACQUISTION TECHNIQUE}

\subsection{Introduction}

This chapter presents the idea that forms the principal contribution of this thesis, namely the adaptive acquisition technique. The concept behind the technique is described in Section 3.2. In Section 3.3 we explicitly state the requirements for our decision functions, which are defined in detail in Section 3.4. Finally, Section 3.5 concludes the chapter.

\subsection{The Adaptive Acquisition Concept}

Before we explore the details of the adaptive acquisition technique, we should first briefly describe the conventional PNF acquisition method. The probe starts in one corner of a predefined scan plane and travels along one dimension, acquiring samples at a given sampling frequency. When the probe reaches the scan plane boundary it changes direction and travels for one sample along the other dimension, changes direction again and travels back along the original dimension. This process is repeated until the complete scan plane has been traversed. However, most PNF scanners can be programmed to make the probe follow other loci.

For the adaptive acquisition technique we assume that we have an existing conventional PNF facility and operation. The goal is to reduce near-field data acquisition time by requiring fewer data points. We recognised that the far-zone field computation time is very small compared to the data acquisition time even if such computation is done 
repeatedly (while data is being acquired). This fact does not appear to have been previously recognised or exploited by others. The proposal is that such computations be used as part of an adaptive algorithm intent on reducing the amount of data that has to be acquired, and hence the overall antenna testing time. This work represents a first step in moving towards building feedback/adaptivity into near-field measurements.

Figure 3.2-1 shows, merely for purposes of illustration, an antenna array of $5 \times 5$ elements, superimposed on which is an array of sampling points over a planar surface in front of the antenna. The adaptive technique utilises a probe that moves on a rectangular spiral type locus ${ }^{15}$ (as indicated), encompassing an increasingly large number of sampling points as it moves. Imagine that we have a collection of sampled near-field values at the $(n-1)^{\text {th }}$ stage, as indicated by the locus (- - ) in Figure 3.2-1. We then let the probe continue along its data acquisition path until at the $n^{\text {th }}$ stage we have an additional number of data points, as indicated by the locus (- - in Figure 3.2-1. We can define this acquisition process in terms of a number of iterations, $n=0,1,2, \ldots ., N$. We are of course at liberty to define the end of the $n^{\text {th }}$ stage as we wish; indeed we could in the extreme define the acquisition of each new data point as the end of a new iteration (although we will not do this here). We have here indicated a specific probe locus, which is the one that is examined in this thesis. However, the method is equally applicable to other probe loci.

\footnotetext{
${ }^{15}$ This rectangular spiral acquisition path does not require any modifications to the mechanical system for moving the probe, as it only moves in either the $\mathrm{x}$ or $\mathrm{y}$ dimension. Developing the software to control the probe in the proposed method is the only upgrade that would be necessary, and is a relatively minor task.
} 


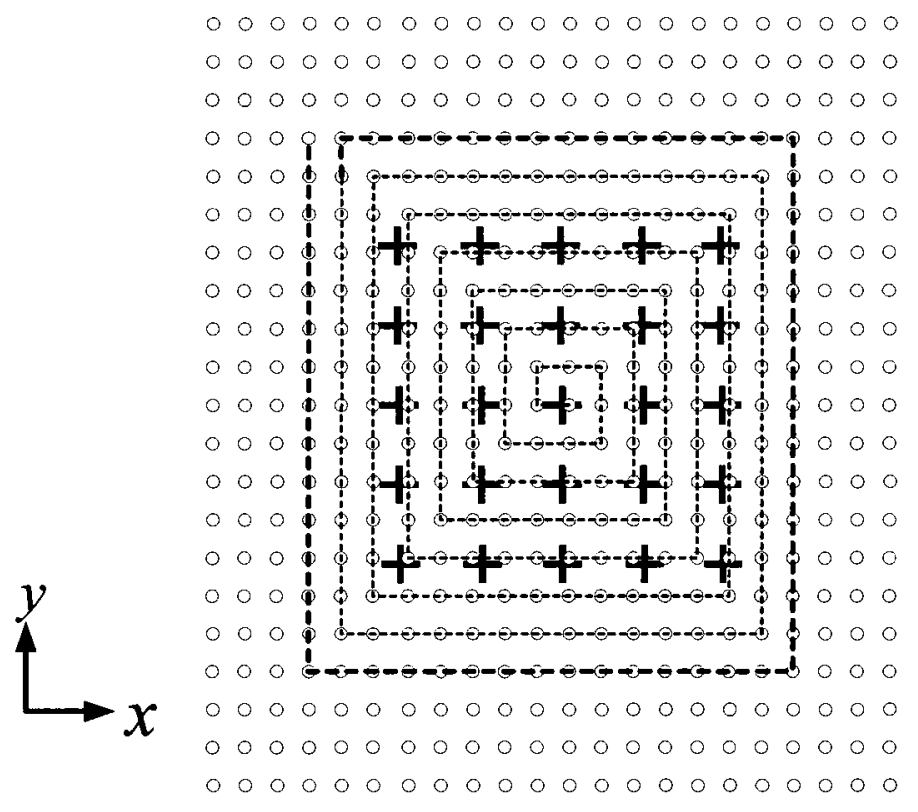

Figure 3.2-1 Sketch showing $5 \times 5$ element array (+), possible sampling points (o), and probe paths after $(n-1)^{\text {th }}(--)$ and $n^{\text {th }}(---)$ iterations.

At the end of each scan iteration we need to calculate decision functions to decide whether or not to terminate the PNF data acquisition. If we can define such decision functions so that they provide a measure of the accuracy with which an identified performance index (eg. directivity of the main beam; maximum sidelobe level; root-meansquare sidelobe level) is being determined at each scan iteration, then the PNF data acquisition can be terminated when the desired accuracy is reached. In this way we can acquire the smallest amount of data in order to achieve the desired accuracy of the selected performance measure of the AUT, in this way minimising the amount of testing time.

Most PNF probes have a single linear polarization which means they can only acquire one component of the near-field at a time. The near-field over the full scan plane is acquired in one polarization first (say $\mathrm{E}_{\mathrm{y}}$ ). The probe is then physically rotated by $90^{\circ}$ and the data is reacquired over the same scan plane for the orthogonal polarization $\left(\mathrm{E}_{\mathrm{x}}\right)$. If 
the adaptive method is applied using such a single polarized probe then one would scan outwards (with the probe set to the dominant polarization) until a decision function recommends termination of the scan. Then the reverse path would be scanned by spiralling inwards with the probe set to the orthogonal polarization. In some cases, indeed in those cases where test times are of the essence, engineers often use dual-polarized probes ${ }^{16}$, which acquire both components simultaneously and then only a single outward scan would be needed.

Probe correction need not be done during the adaptive procedure. Only once the final scan size has been reached, and data acquisition terminated, do we need to do probe correction to obtain the final radiation pattern. Probe correction could be done at each iteration step because it adds negligible computation time, but is not necessary.

\subsection{Decision Function Requirements}

There are two general requirements that a decision function must satisfy:

- It must have a behaviour that allows us to boldly apply a stopping-criterion (that is, take an unambiguous decision that the PNF data acquisition may be terminated).

- It must be such that, when we apply a stopping-criterion, we end up with what we actually want.

\footnotetext{
${ }^{16}$ In fact, in some cases only the dominant polarization is measured if precise cross-polarization levels are not required.
} 


\subsection{Decision Function Definitions}

There are three categories of decision functions defined in this thesis ${ }^{17}$ :

- DFE - those based on the differences of the far-zone radiation patterns obtained from the $n^{\text {th }}$ and the $(n-1)^{\text {th }}$ near-field scan iterations

- DFD - those based on the differences of the antenna directivity determined from the near-field data of the $n^{\text {th }}$ and the $(n-1)^{\text {th }}$ scan iterations

- DFN - those based on an estimate of the fractional truncation errors within the far-zone region of validity for a given scan iteration.

Each category then contains three types of calculations:

- 1 - average of the parameter calculated

- 2 - maximum of the parameter calculated

- 3 - value of the parameter calculated at particular locations in the radiation pattern (that is, in a particular direction)

For example, we will refer to the decision function that evaluates the maximum directivity difference as DFD2. We will also refer to the group of DFE decision functions as DFEn, and similarly for DFD and DFN.

The details of the decision functions are described in depth in the following sections.

\footnotetext{
${ }^{17}$ These will be determined from the complete three-dimensional pattern data an not just principal pattern cut data.
} 


\subsubsection{DFE1 - Average of E-Field Differences}

The first decision function, DFE1, is calculated in co- and cross-polarized components as:

$$
\begin{gathered}
F_{n}^{c o}=20 \log \left\{\frac{\sum_{i=1}^{I_{\max }} \sum_{j=1}^{J_{\max }}\left\{w_{c o}\left(\theta_{i}, \phi_{j}\right)\left|f_{c o}^{n}\left(\theta_{i}, \phi_{j}\right)\right|\right\}}{I_{\max } J_{\max }}\right\} \\
F_{n}^{c r}=20 \log \left\{\frac{\sum_{i=1} \sum_{j=1}^{I_{\max }}\left\{w_{c r}\left(\theta_{i}, \phi_{j}\right) \mid f_{c r}^{n}\left(\theta_{i}, \phi_{j}\right)\right\}}{I_{\max } J_{\max }}\right\}
\end{gathered}
$$

where the value of $f_{c o}^{n}\left(\theta_{i}, \phi_{j}\right)$ is found by subtracting the magnitude of the normalized copolarized far-zone E-field from the current scan iteration, $\widetilde{E}_{c o}^{n}\left(\theta_{i}, \phi_{j}\right)$, by the magnitude of the normalized co-polarized far-zone E-field from the previous iteration, $\widetilde{E}_{c o}^{n-1}\left(\theta_{i}, \phi_{j}\right)$,

$$
f_{c o}^{n}\left(\theta_{i}, \phi_{j}\right)=\widetilde{E}_{c o}^{n}\left(\theta_{i}, \phi_{j}\right)-\widetilde{E}_{c o}^{n-1}\left(\theta_{i}, \phi_{j}\right)
$$

The normalized co-polarized far-zone E-fields for the current, $n^{\text {th }}$, and previous, $(n-1)^{\text {th }}$, scan iterations are found using: 


$$
\begin{aligned}
& \widetilde{E}_{c o}^{n}\left(\theta_{i}, \phi_{j}\right)=\frac{\left|E_{c o}^{n}\left(\theta_{i}, \phi_{j}\right)\right|}{E_{c o, N o r m}^{n}\left(\theta_{o}, \phi_{o}\right)} \\
& \widetilde{E}_{c o}^{n-1}\left(\theta_{i}, \phi_{j}\right)=\frac{\left|E_{c o}^{n-1}\left(\theta_{i}, \phi_{j}\right)\right|}{E_{c o, N o r m}^{n}\left(\theta_{o}, \phi_{o}\right)} .
\end{aligned}
$$

The normalization factor, $E_{c o, N o r m}^{n}\left(\theta_{o}, \phi_{o}\right)$, is the same for the $n^{\text {th }}$ and $(n-1)^{\text {th }}$ iteration and is simply the maximum value of $\left|E_{c o}^{n}\left(\theta_{i}, \phi_{j}\right)\right|$ for the $n^{\text {th }}$ scan iteration,

$$
E_{c o, N o r m}^{n}\left(\theta_{o}, \phi_{o}\right)=\max \left(\left|E_{c o}^{n}\left(\theta_{i}, \phi_{j}\right)\right|\right)
$$

The values for the cross-polarized case are defined in a similar way using:

$$
\begin{gathered}
f_{c r}^{n}\left(\theta_{i}, \phi_{j}\right)=\widetilde{E}_{c r}^{n}\left(\theta_{i}, \phi_{j}\right)-\widetilde{E}_{c r}^{n-1}\left(\theta_{i}, \phi_{j}\right) \\
\tilde{E}_{c r}^{n}\left(\theta_{i}, \phi_{j}\right)=\frac{\left|E_{c r}^{n}\left(\theta_{i}, \phi_{j}\right)\right|}{E_{c o, N o r m}\left(\theta_{o}, \phi_{o}\right)} \\
\tilde{E}_{c r}^{n-1}\left(\theta_{i}, \phi_{j}\right)=\frac{\left|E_{c r}^{n-1}\left(\theta_{i}, \phi_{j}\right)\right|}{E_{c o, N o r m}^{n}\left(\theta_{o}, \phi_{o}\right)}
\end{gathered}
$$

and $E_{c o, N o r m}^{n}\left(\theta_{o}, \phi_{o}\right)$ is defined as in 3.4.1-6. 
The decision function also contains two input variables that can be used to:

- limit the region of the far-zone for use in the decision function based on constraining the far-zone elevation and azimuth angles, $\theta$ and $\phi$

- apply a lower limit for the values of $\left|\widetilde{E}_{c o}^{n}\left(\theta_{i}, \phi_{j}\right)\right|$ and $\left|\widetilde{E}_{c r}^{n}\left(\theta_{i}, \phi_{j}\right)\right|$ which essentially sets the dynamic range for the data used in the decision function, given in $\mathrm{dB}$

The co-polarized lower limit, $P_{D F E, c o}$ in $\mathrm{dB}$, for this decision function is set so that any value of the normalized far-zone field, $\mid \widetilde{E}_{c o}^{n}\left(\theta_{i}, \phi_{j}\right)$, that is less than $P_{D F E, c o}$ is set to $P_{D F E, c o}$,

$$
\left|\tilde{E}_{c o}^{n}\left(\theta_{i}, \phi_{j}\right)\right|=\left\{\begin{array}{ll}
\left|\tilde{E}_{c o}^{n}\left(\theta_{i}, \phi_{j}\right)\right| & \text { if } 20 \log \left|\tilde{E}_{c o}^{n}\left(\theta_{i}, \phi_{j}\right)\right| \geq P_{D F E, c o} \\
10^{P_{D F E, c o} / 20} & \text { Otherwise }
\end{array}\right\} .
$$

A similar, but not necessarily the same, lower limit can be applied to the cross-polarized data such that,

$$
\left|\tilde{E}_{c r}^{n}\left(\theta_{i}, \phi_{j}\right)\right|=\left\{\begin{array}{ll}
\left|\tilde{E}_{c r}^{n}\left(\theta_{i}, \phi_{j}\right)\right| & \text { if } 20 \log \left|\tilde{E}_{c r}^{n}\left(\theta_{i}, \phi_{j}\right)\right| \geq P_{D F E, c r} \\
10^{P_{D F E, c r} / 20} & \text { Otherwise }
\end{array}\right\} .
$$


A similar method is used to apply $P_{D F E, c o}$ and $P_{D F E, c o}$ to $\left|\tilde{E}_{c o}^{n-1}\left(\theta_{i}, \phi_{j}\right)\right|$ and $\left|\tilde{E}_{c r}^{n-1}\left(\theta_{i}, \phi_{j}\right)\right|$. Setting appropriate values for $P_{D F E, c o}$ and $P_{D F E, c o}$ enables us to ignore the differences between the $n^{\text {th }}$ and $(n-1)^{\text {th }}$ scan iterations that occur at very low $\mathrm{dB}$ values, such as in the pattern nulls.

Figure 3.4-1 shows the effect of setting the $P_{D F E, c o}$ on the co-polarized far-zone radiation pattern of a Horn antenna. The left image shows the original radiation pattern with no $P_{D F E, c o}$ set, while in the right image $P_{D F E, c o}=-50 \mathrm{~dB}$.
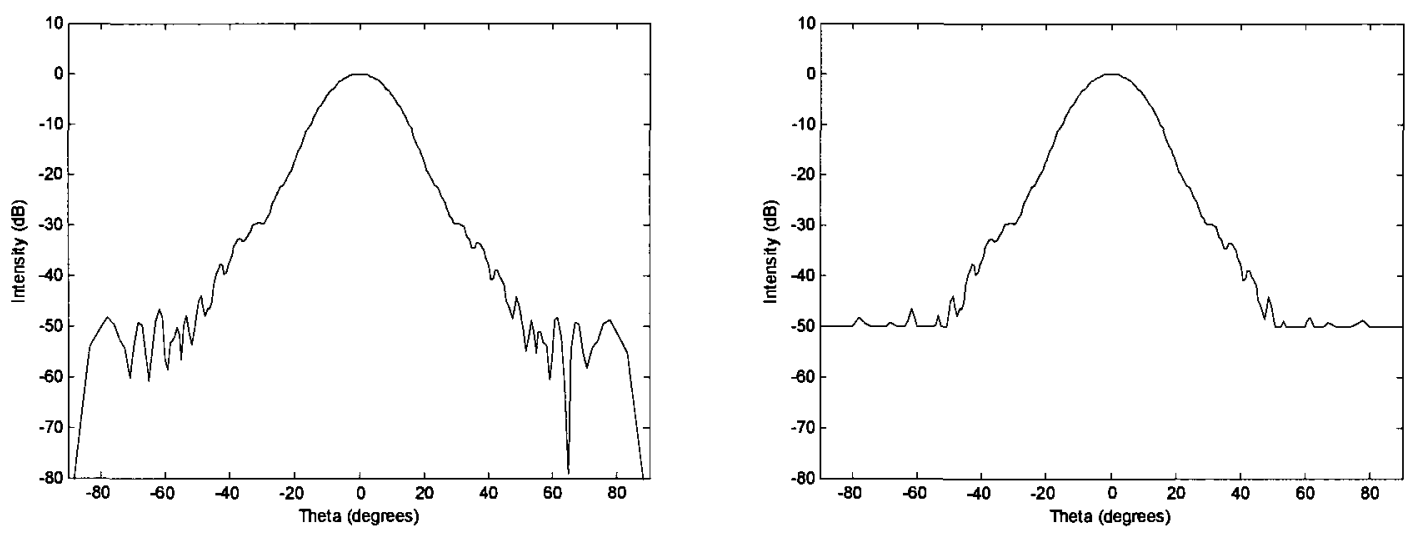

Figure 3.4-1: Effect of $P_{D F E, c o}$ on the calculated far-zone E-field for the $\phi=0$ cut of a Horn antenna. The left image does not have a lower limit applied, while in the right image $P_{D F E, c o}=\mathbf{- 5 0} \mathbf{~ d B}$.

The second set of input variables are weighting functions, $w_{c o}\left(\theta_{i}, \phi_{j}\right)$ and $w_{c r}\left(\theta_{i}, \phi_{j}\right)$ for the co- and cross-polarized cases, that can used to scale the far-zone radiation patterns by setting a factor for each $\theta_{i}$ and $\phi_{j}$. In this thesis, the weighting functions have been implemented in such a way as to set a far-zone region of interest by 
limiting the values of $\theta$. Any values of the normalized far-zone that are equal to or lie outside the set $\theta_{\min }$ and $\theta_{\max }$ boundary are set to 0 and not included in the decision function calculation. This has been accomplished by creating a mask that has a value of 1 inside the set angular region, and 0 on the boundary and outside. Strictly speaking, the $w_{c o}\left(\theta_{i}, \phi_{j}\right)$ and $w_{c r}\left(\theta_{i}, \phi_{j}\right)$ weighting functions are based on both $\theta$ and $\phi$, however in practice only $\theta$ will be used.

$$
\begin{aligned}
& w_{c o}\left(\theta_{i}, \phi_{j}\right)=\left\{\begin{array}{ll}
1 & \text { if } \theta_{i}>\theta_{c o, \min } \text { and } \theta_{i}<\theta_{c o, \max } \text { and } \phi_{j}>\phi_{c o, \min } \text { and } \phi_{j}<\phi_{c o, \max } \\
0 & \text { otherwise }
\end{array}\right\} \\
& w_{c r}\left(\theta_{i}, \phi_{j}\right)=\left\{\begin{array}{ll}
1 & \text { if } \theta_{i}>\theta_{c r, \min } \text { and } \theta_{i}<\theta_{c r, \max } \text { and } \phi_{j}>\phi_{c r, \min } \text { and } \phi_{j}<\phi_{c r, \max } \\
0 & \text { otherwise }
\end{array}\right\}
\end{aligned}
$$

Figure 3.4-2 shows the effect of setting of the weighting function $w_{c o}\left(\theta_{i}, \phi_{j}\right)$.

The left image shows the original, unconstrained co-polarized far-field radiation pattern of a Horn antenna. In the right image we have set $\theta_{c o, \min }$ to $-30^{\circ}$ and $\theta_{c o, \max }$ to $30^{\circ}$, essentially setting the angle off broadside to be $\pm 30^{\circ}$. 

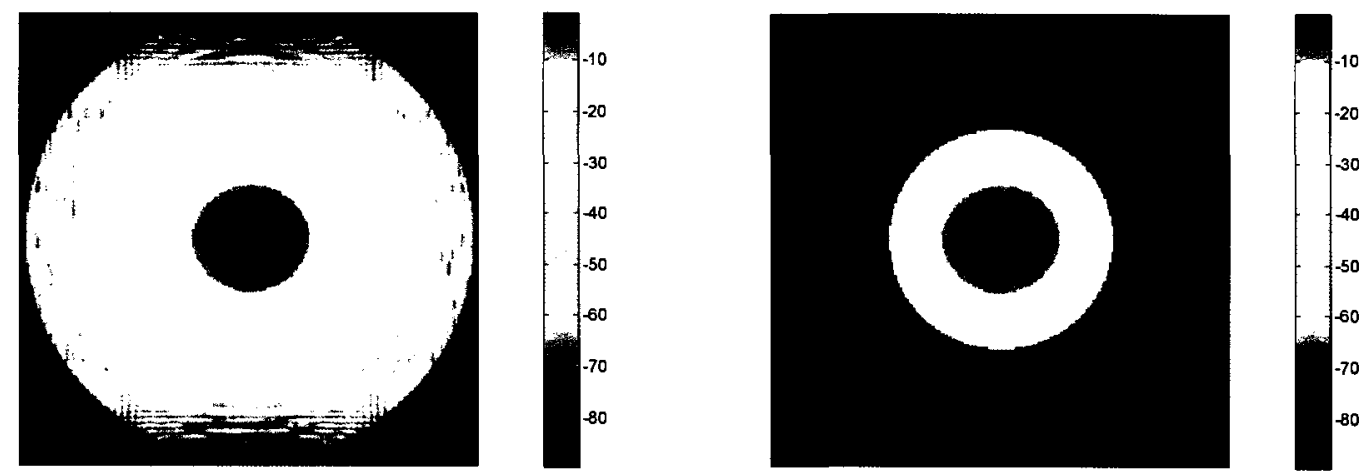

Figure 3.4-2: Effect of $w_{c o}\left(\theta_{i}, \phi_{j}\right)$ on the far-zone E-field of a Horn antenna. In the left image, no weighting function has been applied, while in the right image $\theta_{c, \text { min }}=$ $\mathbf{- 3 0}^{\circ}$ and $\theta_{c o, \max }=\mathbf{3 0}^{\circ}$.

If the normalized radiation pattern magnitude in direction $\left(\theta_{i}, \phi_{j}\right)$ is $E_{d B}$, and we wish the uncertainty there to be $\pm \Delta_{d B}$, then we require :

$$
20 \log \left|f_{c o}^{n}\left(\theta_{i}, \phi_{j}\right)\right| \leq E_{d B}+20 \log \left\{1-10^{-\Delta_{d B} / 20}\right\}
$$

Table 3.4-1 shows the values of $20 \log \left|f_{c o}^{n}\left(\theta_{i}, \phi_{j}\right)\right|$ for various combinations of $E_{d B}$ and $\Delta_{d B} ;$ these are also plotted in Figure 3.4-3. 
Table 3.4-1: DFEn values required for an uncertainty, $\pm \Delta_{d B}$, at a normalized pattern level, $E_{d B}$

\begin{tabular}{|l|l|l|l|l|l|l|}
\hline \multirow{2}{*}{ Uncertainty $\Delta_{d B}$} & \multicolumn{5}{|c|}{ Level of Normalised Pattern $E_{d B}$} \\
\cline { 2 - 7 } & $\mathbf{0}$ & $\mathbf{- 1 0}$ & $\mathbf{- 1 5}$ & $\mathbf{- 2 0}$ & $\mathbf{- 2 5}$ & $\mathbf{- 3 0}$ \\
\hline $\mathbf{0 . 1}$ & -38.8 & -48.8 & -53.8 & -58.8 & -63.8 & -68.8 \\
\hline $\mathbf{0 . 2}$ & -32.8 & -42.8 & -47.8 & -52.8 & -57.8 & -62.8 \\
\hline $\mathbf{0 . 5}$ & -25.5 & -35.0 & -40.0 & -45.0 & -50.0 & -55.0 \\
\hline $\mathbf{1}$ & -19.3 & -29.3 & -34.3 & -39.3 & -44.3 & -49.3 \\
\hline $\mathbf{1 . 5}$ & -16.0 & -26.0 & -31.0 & -36.0 & -41.0 & -46.0 \\
\hline $\mathbf{2}$ & -13.7 & -23.7 & -28.7 & -33.7 & -38.7 & -43.7 \\
\hline
\end{tabular}

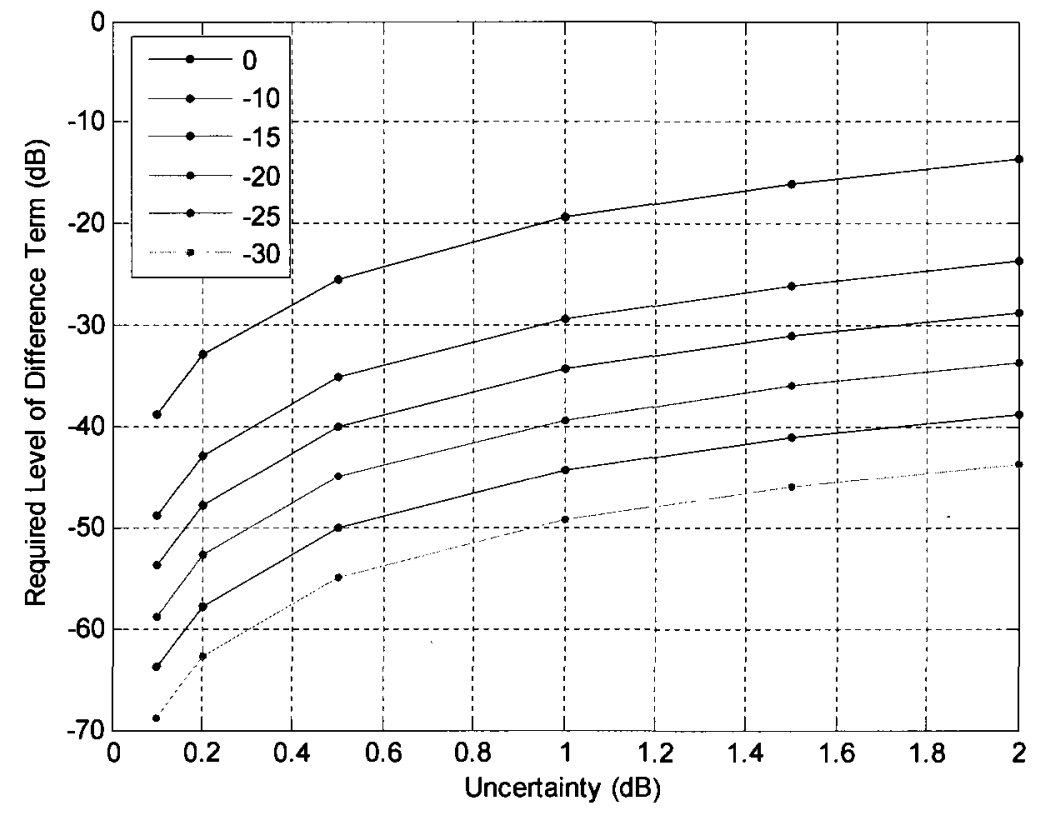

Figure 3.4-3: Plot showing DFEn values required for an uncertainty, $\pm \Delta_{d B}$, at a particular normalized pattern level, $E_{d B}$ 


\subsubsection{DFE2 - Maximum of E-Field Differences}

The DFE2 decision function focuses on the maximum difference between the farzone radiation E-field from the current and previous PNF scan iterations, and is separately in the co-polarized and cross-polarized cases as:

$$
\begin{aligned}
& F_{n}^{c o}=20 \log \left\{\max \left\{w_{c o}\left(\theta_{i}, \phi_{j}\right)\left|f_{c o}^{n}\left(\theta_{i}, \phi_{j}\right)\right|\right\}\right\} \\
& F_{n}^{c r}=20 \log \left\{\max \left\{w_{c r}\left(\theta_{i}, \phi_{j}\right)\left|f_{c r}^{n}\left(\theta_{i}, \phi_{j}\right)\right|\right\}\right\}
\end{aligned}
$$

The definitions of the components of expressions (3.4.2-1) and (3.4.2-2) are the same as for DFE1 in Section 3.4.1.

The lower limit, $P_{D F E, c o}$ and $P_{D F E, c r}$, as well as the weighting functions $w_{c o}\left(\theta_{i}, \phi_{j}\right)$ and $w_{c r}\left(\theta_{i}, \phi_{j}\right)$ are also applied to DFE2 as described in the section on DFE1. It should be noted that the maximum difference is found after the $w_{c o}\left(\theta_{i}, \phi_{j}\right)$ and $w_{c r}\left(\theta_{i}, \phi_{j}\right)$ have been multiplied with the $\left|f_{c o}^{n}\left(\theta_{i}, \phi_{j}\right)\right|$ and $\left|f_{c r}^{n}\left(\theta_{i}, \phi_{j}\right)\right|$.

\subsubsection{DFE3 - Difference of E-Fields at a Defined Point}

This decision function is related to DFE2, except that instead of evaluating the decision function at the maximum difference between the current and previous scan iteration, DFE3 evaluates the difference only at some pre-defined point, $\left(\theta_{I}, \phi_{J}\right)$. DFE3 is expressed in co-polarized and cross-polarized form as: 


$$
\begin{aligned}
& F_{n}^{c o}=20 \log \left\{w_{c o}\left(\theta_{c o, I}, \phi_{c o, J}\right)\left|f_{c o}^{n}\left(\theta_{c o, I}, \phi_{c o, J}\right)\right|\right\} \\
& F_{n}^{c r}=20 \log \left\{w_{c r}\left(\theta_{c r, I}, \phi_{c r, J}\right)\left|f_{c r}^{n}\left(\theta_{c r, I}, \phi_{c r, J}\right)\right|\right\}
\end{aligned}
$$

where $\left(\theta_{c o, I}, \phi_{c o, J}\right)$ and $\left(\theta_{c r, I}, \phi_{c r, J}\right)$ are the pre-defined far-zone locations for the copolarized and cross-polarized components, which do not necessarily have to be the same point. The definitions of the other components of expressions (3.4.3-1) and (3.4.3-2) are the same as in Section 3.4.1 on the DFE1.

The lower limits for DFE3, $P_{D F E, c o}$ and $P_{D F E, c r}$, as well as the weighting functions $w_{c o}\left(\theta_{i}, \phi_{j}\right)$ and $w_{c r}\left(\theta_{i}, \phi_{j}\right)$ are also applied as described in Section 3.4.1.

\subsubsection{DFD1 - Average Directivity Differences}

DFD1 follows the same procedures as DFE1, except that the directivity of the AUT is used instead of the far-zone radiated E-fields. The co-polarized and crosspolarized components of the decision function are evaluated as:

$$
F_{n}^{c o}=\left\{\frac{\sum_{i=1}^{I_{\max }} \sum_{j=1}^{J_{\max }}\left\{w_{c o}\left(\theta_{i}, \phi_{j}\right)\left|f_{c o}^{n}\left(\theta_{i}, \phi_{j}\right)\right|\right\}}{I_{\max } J_{\max }}\right\}
$$




$$
F_{n}^{c r}=\left\{\frac{\sum_{i=1}^{I_{\max }} \sum_{j=1}^{J_{\max }}\left\{w_{c r}\left(\theta_{i}, \phi_{j}\right)\left|f_{c r}^{n}\left(\theta_{i}, \phi_{j}\right)\right|\right\}}{I_{\max } J_{\max }}\right\} .
$$

Directivity is an absolute performance measure itself, and is always expressed in $\mathrm{dBi}$. We want to compare its value in $\mathrm{dBi}$ from the $n^{\text {th }}$ and the $(n-1)^{\text {th }}$ iterations to determine if we are homing in on some final value. As such, the magnitude of the directivity from the current PNF scan iteration is subtracted by the magnitude of the directivity from the previous scan iteration for both the co-polarized and cross-polarized partial directivities,

$$
\begin{aligned}
& f_{c o}^{n}\left(\theta_{i}, \phi_{j}\right)=|10 \log | D_{c o}^{n}\left(\theta_{i}, \phi_{j}\right)||-|10 \log | D_{c o}^{n-1}\left(\theta_{i}, \phi_{j}\right)|| \\
& f_{c r}^{n}\left(\theta_{i}, \phi_{j}\right)=|10 \log | D_{c r}^{n}\left(\theta_{i}, \phi_{j}\right)||-|10 \log | D_{c r}^{n-1}\left(\theta_{i}, \phi_{j}\right)|| .
\end{aligned}
$$

In general, the directivity $D(\theta, \phi)$ is given by [1]:

$$
D(\theta, \phi)=4 \pi \frac{U(\theta, \phi)}{P_{t o t}}
$$

The function $U(\theta, \phi)$ is the power radiated by the AUT per unit solid angle and $P_{t o t}$ is the total radiated power, 


$$
\begin{aligned}
& U(\theta, \phi)=\frac{1}{2 \eta}\left[\left.\left|E_{\theta}(\theta, \phi)^{2}+\right| E_{\phi}(\theta, \phi)\right|^{2}\right] \\
& P_{t o t}=\int_{0}^{\pi}\left[\int_{0}^{2 \pi} U(\theta, \phi) d \phi\right] \sin \theta d \theta .
\end{aligned}
$$

This decision function uses the partial directivities, $D_{c o}(\theta, \phi)$ and $D_{c r}(\theta, \phi)$, which are defined as,

$$
\begin{gathered}
D_{c o}(\theta, \phi)=4 \pi \frac{U_{c o}(\theta, \phi)}{P_{t o t}} \\
U_{c o}(\theta, \phi)=\frac{1}{2 \eta}\left[\left|E_{c o}(\theta, \phi)\right|^{2}\right]
\end{gathered}
$$

for the co-polarized case and

$$
\begin{gathered}
D_{c r}(\theta, \phi)=4 \pi \frac{U_{c r}(\theta, \phi)}{P_{t o t}} \\
U_{c r}(\theta, \phi)=\frac{1}{2 \eta}\left[E_{c r}(\theta, \phi)^{2}\right]
\end{gathered}
$$

for the cross-polarized partial directivity.

$P_{t o t}$ is evaluated in [2] as: 


$$
\begin{gathered}
P_{t o t}=\frac{1}{2 k \eta_{o}} \operatorname{Re}\left\{\iint_{k_{x}^{2}+k_{y}^{2} \leq k^{2}} \frac{\left|A_{x}\left(k_{x}, k_{y}\right)\right|^{2}\left(k-k_{y}^{2}\right)}{k_{z}} d k_{x} d k_{y}+\iint_{k_{x}^{2}+k_{y}^{2} \leq k^{2}} \frac{\left|A_{y}\left(k_{x}, k_{y}\right)\right|^{2}\left(k-k_{x}^{2}\right)}{k_{z}} d k_{x} d k_{y}\right. \\
\left.+\iint_{k_{x}^{2}+k_{y}^{2} \leq k^{2}} \frac{k_{x} k_{y}\left[A_{x}\left(k_{x}, k_{y}\right) A_{y}^{*}\left(k_{x}, k_{y}\right)+A_{x}^{*}\left(k_{x}, k_{y}\right) A_{y}\left(k_{x}, k_{y}\right)\right]}{k_{z}} d k_{x} d k_{y}\right\}
\end{gathered}
$$

The integrals in the above expression were implemented in MATLAB using Simpson's numerical integration [3], which was based on the MATLAB function trapz, and can be found in Appendix A.

Similar to the previous decision functions, there is also a limiting factor for the co- and cross-polarized cases that can be applied to the directivity decision functions. However the values of $P_{D F D, c o}$ and $P_{D F D, c r}$ specify a limit below the maximum directivity for the current scan iteration,

$$
\begin{aligned}
& D_{c o}^{n}(\theta, \phi)=\left\{\begin{array}{cc}
D_{c o}^{n}(\theta, \phi), & \text { if } D_{c o}^{n}(\theta, \phi) \geq \operatorname{Max}\left(D_{c o}^{n}(\theta, \phi)\right)-P_{D F D, c o} \\
D_{c o}^{n}(\theta, \phi)-P_{D F D, c o}, & \text { otherwise }
\end{array}\right\} \\
& D_{c r}^{n}(\theta, \phi)=\left\{\begin{array}{cc}
D_{c r}^{n}(\theta, \phi), & \text { if } D_{c r}^{n}(\theta, \varphi) \geq \operatorname{Max}\left(D_{c r}^{n}(\theta, \varphi)\right)-P_{D F D, c r} \\
D_{c r}^{n}(\theta, \phi)-P_{D F D, c r}, & \text { otherwise }
\end{array}\right\} \text {. }
\end{aligned}
$$

The same maximum directivity found for $D_{c o}^{n}(\theta, \phi)$ is used for $D_{c o}^{n-1}(\theta, \phi)$ when applying $P_{D F D, c o}$ to the previous scan iteration, and similarly for the cross-polarized case. 
The weighting functions, $w_{c o}\left(\theta_{i}, \phi_{j}\right)$ and $w_{c r}\left(\theta_{i}, \phi_{j}\right)$ are applied using the same method as described in Section 3.4.1 on the DFE1.

\subsubsection{DFD2 - Maximum of Directivity Differences}

DFD2 follows the same method as DFE2, except that instead of the maximum difference in the far-zone E-fields, the maximum difference in the directivity between the current and previous scan iterations are used in the decision function. The co-polarized and cross-polarized components of DFD2 are defined as:

$$
\begin{aligned}
& F_{n}^{c o}=\left\{\max \left(w_{c o}\left(\theta_{i}, \phi_{j}\right)\left|f_{c o}^{n}\left(\theta_{i}, \phi_{j}\right)\right|\right)\right\} \\
& F_{n}^{c r}=\left\{\max \left(w_{c r}\left(\theta_{i}, \phi_{j}\right)\left|f_{c r}^{n}\left(\theta_{i}, \phi_{j}\right)\right|\right)\right\}
\end{aligned}
$$

The derivation of $f_{c o}^{n}\left(\theta_{i}, \phi_{j}\right)$ and $f_{c r}^{n}\left(\theta_{i}, \phi_{j}\right)$ follow the same procedure as DFD1. The application of the weighting functions $w_{c o}\left(\theta_{i}, \phi_{j}\right)$ and $w_{c r}\left(\theta_{i}, \phi_{j}\right)$ is as described in Section 3.4.1, and the limits $P_{D F D, c o}$ and $P_{D F D, c r}$ are as described in Section 3.4.5. 


\subsubsection{DFD3 - Difference of Directivity at a Defined Point}

DFD3 follows the same derivation as DFE3, except that the calculated directivity of the antenna is used instead of the E-fields. As with DFE3, DFD3 is evaluated at some pre-defined far-zone point, $\left(\theta_{I}, \phi_{J}\right)$, and the co- and cross-polarized components are:,

$$
\begin{aligned}
& F_{n}^{c o}=\left\{w_{c o}\left(\theta_{c o, I}, \phi_{c o, J}\right)\left|f_{c o}^{n}\left(\theta_{c o, I}, \phi_{c o, J}\right)\right|\right\} \\
& F_{n}^{c r}=\left\{w_{c r}\left(\theta_{c r, I}, \phi_{c r, J}\right)\left|f_{c r}^{n}\left(\theta_{c r, I}, \phi_{c r, J}\right)\right|\right\}
\end{aligned}
$$

where $\left(\theta_{c o, I}, \phi_{c o, J}\right)$ and $\left(\theta_{c r, I}, \phi_{c r, J}\right)$ are the pre-defined far-zone locations for the copolarized and cross-polarized components, and are not necessarily the same point. The definitions for the remaining elements of $F_{n}$ are the same as in Section 3.4.4.

The application of the lower limits $P_{D F D, c o}$ and $P_{D F D, c r}$, and the weighting functions $w_{c o}\left(\theta_{c o, I}, \phi_{c o, J}\right)$ and $w_{c r}\left(\theta_{c r, I}, \phi_{c r, J}\right)$, are again the same as in Section 3.4.4.

\subsubsection{DFN1 - Average of Fractional Plane Wave Spectrum Error}

The act of reducing the scan plane size will ultimately result in truncation errors in the valid region of the calculated far-zone field, and a decision function based on an estimate of the fractional plane wave spectrum error due to scan plane truncation is described in this section. 
The fractional plane wave spectrum error measurement is adapted from $[4]^{18}$, such that the fractional error in the valid region due to scan area truncation for the current scan iteration is given in the $\mathrm{x}$ and $\mathrm{y}$ components as:

$$
\begin{aligned}
& \frac{\left|\Delta A_{x}^{n}\left(k_{x}, k_{y}\right)\right|}{\left|A_{x}^{n}\left(k_{x}, k_{y}\right)\right|} \leq \lambda \frac{\left|\int_{C} E_{x}^{n}\left(x^{\prime}, y^{\prime}\right) e^{j k_{x} x^{\prime}} e^{j k_{y} y} d \ell^{\prime}\right|}{2 \pi \cos \gamma_{\max }\left|\iint_{S} E_{x}^{n}\left(x^{\prime}, y^{\prime}\right) e^{j k_{x} x^{\prime}} e^{j k_{y} y} d x^{\prime} d y^{\prime}\right|} \\
& \frac{\left|\Delta A_{y}^{n}\left(k_{x}, k_{y}\right)\right|}{\left|A_{y}^{n}\left(k_{x}, k_{y}\right)\right|} \leq \lambda \frac{\left|\int_{C} E_{y}^{n}\left(x^{\prime}, y^{\prime}\right) e^{j k_{x} x^{\prime}} e^{j k_{y} y} d \ell^{\prime}\right|}{2 \pi \cos \gamma_{\max }\left|\iint_{S} E_{y}^{n}\left(x^{\prime}, y^{\prime}\right) e^{j k_{x} x^{\prime}} e^{j k_{y} y} d x^{\prime} d y^{\prime}\right|} .
\end{aligned}
$$

The values of $E_{x}^{n}\left(x^{\prime}, y^{\prime}\right)$ and $E_{y}^{n}\left(x^{\prime}, y^{\prime}\right)$ are the actual sampled PNF data for the $n^{\text {th }}$ iteration, and $\gamma_{\max }$ is the maximum acute angle between the plane of the scan area and any line connecting the edges of the antenna aperture and the edges of the scan area, as illustrated in Figure 3.4-4. One can easily see that the angle $\gamma_{\max }$ is only acute once the aperture of the AUT has been scanned, and is maximum when the largest AUT aperture dimension is used. $\Delta_{x}$ and $\Delta_{y}$ are the near-field sample spacing in wavelengths, and $\lambda$ is the wavelength of the AUT.

${ }^{18}$ There is a typographical error in the denominator of formula (48) in [4]. Correspondence with A. Yaghjian, who originally derived (48) confirmed the corrected version is given in this thesis as (3.4.7-1) and (3.4.7-2). Note that these expressions do not include the $\delta_{x}$ and $\delta_{y}$ that appear in (48) of [4] since we write the denominator as a surface integral and not just a summation. 


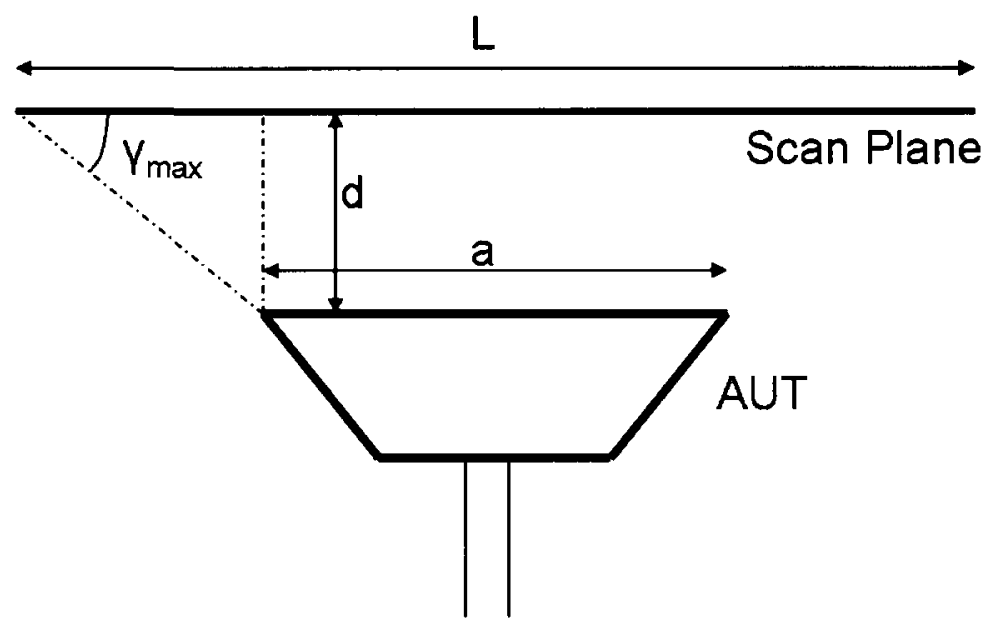

Figure 3.4-4: $\gamma_{\max }$ for DFN1. The maximum acute angle occurs once the aperture has been scanned and when $a$ is the largest AUT aperture dimension.

The numerators in the above expressions are contour integrals along the boundary of the $n^{\text {th }}$ scan iteration. The contour integral in the numerator was evaluated using the four line integrals found in expressions (3.4.7-3) to (3.4.7-6), and as indicated in Figure $3.2-1$. 


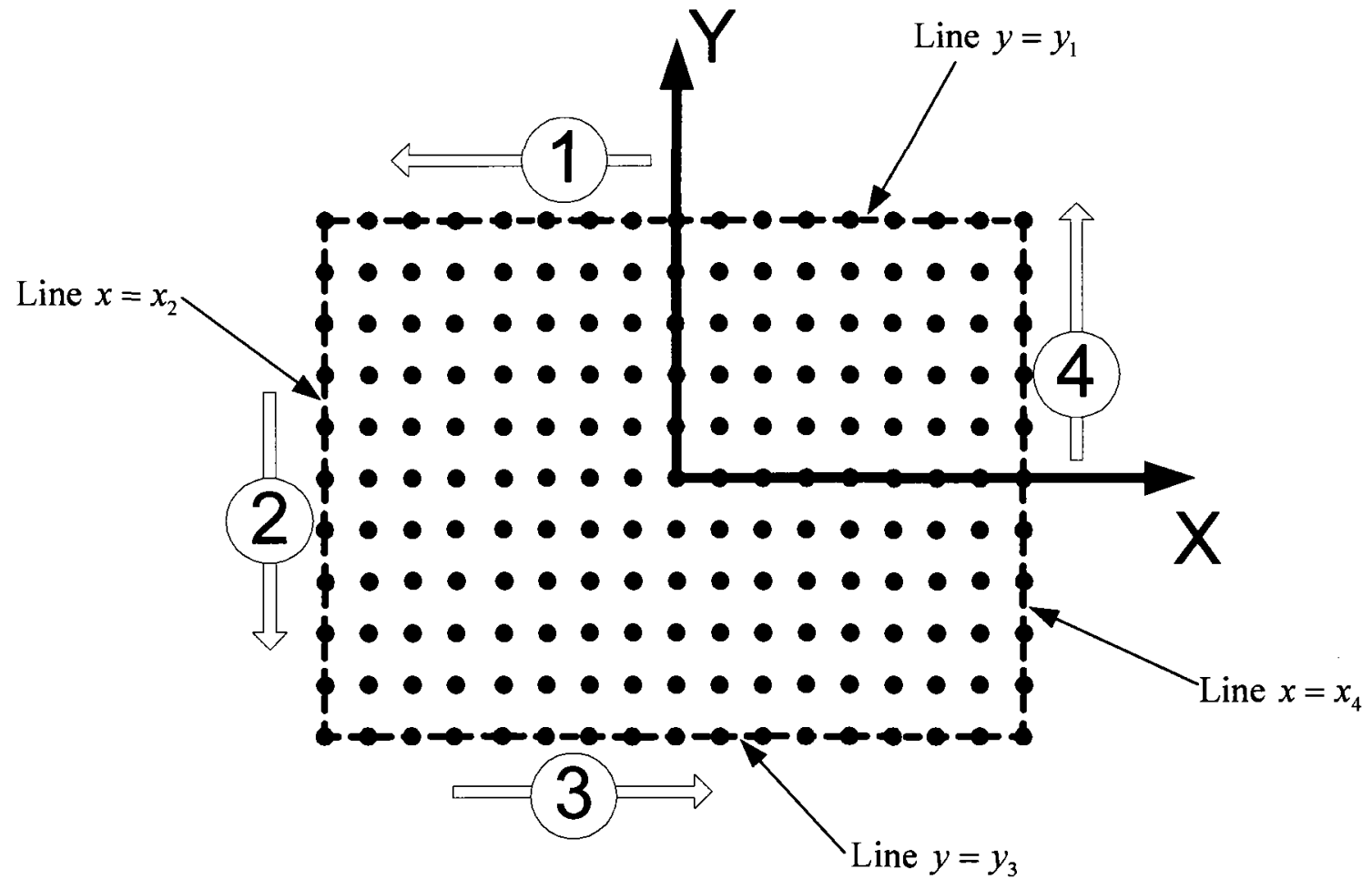

Figure 3.4-5: Sketch showing contour integral in the numerator of expressions 3.4.7-1 and 3.4.7-2

Along Line\#1

$$
F_{x}^{(1)}\left(k_{x}, k_{y}\right)=\int_{C_{1}} E_{x}\left(x^{\prime}, y^{\prime}\right) e^{j k_{x} x^{\prime}} e^{j k_{y} y^{\prime}} d \ell^{\prime}=e^{j k_{y} y_{1}} \int_{x_{4}}^{x_{2}} E_{x}\left(x^{\prime}, y_{1}\right) e^{j k_{x} x^{\prime}} d x^{\prime}=\Delta x e^{j k_{y} y_{1}} \sum_{n=0}^{N-1} E_{x}\left(n \Delta x, y_{1}\right) e^{j k_{x} n \Delta x}
$$

Along Line\#2

$$
F_{x}^{(2)}\left(k_{x}, k_{y}\right)=\int_{C_{2}} E_{x}\left(x^{\prime}, y^{\prime}\right) e^{j k_{x} x^{\prime}} e^{j k_{y} y^{\prime}} d \ell^{\prime}=e^{j k_{x} x_{2}} \int_{y_{1}}^{y_{3}} E_{x}\left(x_{2}, y^{\prime}\right) e^{j k_{y} y^{\prime}} d y^{\prime}=\Delta y e^{j k_{x} x_{2}} \sum_{m=0}^{N-1} E_{x}\left(x_{2}, m \Delta y\right) e^{j k_{y} m \Delta y}
$$




\section{Along Line\#3}

$$
F_{x}^{(3)}\left(k_{x}, k_{y}\right)=\int_{C_{3}} E_{x}\left(x^{\prime}, y^{\prime}\right) e^{j k_{x} x^{\prime}} e^{j k_{y} y^{\prime}} d \ell^{\prime}=e^{j k_{x} y_{3}} \int_{x_{2}}^{x_{4}} E_{x}\left(x^{\prime}, y_{3}\right) e^{j k_{x^{\prime}} x^{\prime}} d x^{\prime}==\Delta x e^{j k_{y} y_{3}} \sum_{n=0}^{N-1} E_{x}\left(n \Delta x, y_{3}\right) e^{j k_{x} \Delta \Delta x}
$$

Along Line\#4

$F_{x}^{(4)}\left(k_{x}, k_{y}\right)=\int_{C_{4}} E_{x}\left(x^{\prime}, y^{\prime}\right) e^{j k_{x} x^{\prime}} e^{j k_{y} y^{\prime}} d \ell^{\prime}=e^{j k_{x} x_{4}} \int_{y_{3}}^{y_{1}} E_{x}\left(x_{4}, y^{\prime}\right) e^{j k_{y} y^{\prime}} d y^{\prime}=\Delta y e^{j k_{x} x_{4}} \sum_{m=0}^{N-1} E_{x}\left(x_{4}, m \Delta y\right) e^{j k_{y} m \Delta y}$

If the $\mathrm{x}$-component is the principal near-field component on the scan plane, then the error in the co-polarized directivity can be estimated [5] in terms of the fractional error by ${ }^{19}$

$$
\Delta D\left(k_{x}, k_{y}\right)= \pm 2 \frac{\left|\Delta A_{x}^{n}\left(k_{x}, k_{y}\right)\right|}{\left|A_{x}^{n}\left(k_{x}, k_{y}\right)\right|} D\left(k_{x}, k_{y}\right)
$$

${ }^{19}$ The expression for the fractional error of the plane wave spectrum is provided in [3]. It is used to obtain Fig.8 of [3]. However, reference [3] omits to say exactly how this needs to be done, something that is not at all obvious. One needs [4] to obtain the relationship between the fractional error of the plane wave spectrum and the directivity uncertainties. 
If $E_{y}$ is the principal component then we of course use $\left|\Delta A_{y}^{n}\right| /\left|A_{y}^{n}\right|$ instead. Note that $\left|\Delta A_{x}^{n}\left(k_{x}, k_{y}\right)\right| /\left|A_{x}^{n}\left(k_{x}, k_{y}\right)\right|$ is not expressed in $\mathrm{dBs}$ in the above expression or in the expressions immediately below. Yaghjian [5] then goes on to show that

$$
\Delta D^{d B}\left(k_{x}, k_{y}\right)=20 \log \left\{1 \pm \frac{\left|\Delta A_{x}^{n}\left(k_{x}, k_{y}\right)\right|}{\left|A_{x}^{n}\left(k_{x}, k_{y}\right)\right|}\right\}
$$

and that for small values of $\left|\Delta A_{x}^{n}\left(k_{x}, k_{y}\right)\right| /\left|A_{x}^{n}\left(k_{x}, k_{y}\right)\right|$, which is the case when we use a decision function to terminate a PNF $\operatorname{scan}^{20}$,

$$
\Delta D^{d B}\left(k_{x}, k_{y}\right)= \pm 8.7 \frac{\left|\Delta A_{x}^{n}\left(k_{x}, k_{y}\right)\right|}{\left|A_{x}^{n}\left(k_{x}, k_{y}\right)\right|}
$$

These expressions give $\Delta D^{d B}$ in any direction (it could be in the direction of the pattern maximum or some sidelobe) with the appropriate value of $\left|\Delta A_{x}^{n}\right| /\left|A_{x}^{n}\right|$ in that direction being used. What this means is that if we have a measured ${ }^{21}$ directivity $D^{d B}$ in some direction then in that direction the actual directivity lies between $D^{d B}+\Delta D^{d B}$ and $D^{d B}-\Delta D^{d B}$. In other words, the length of the error bar (in dBs) in the directivity in this

\footnotetext{
${ }^{20}$ Although the error in the directivity is in dBs, quantity $\left|\Delta A_{x}^{n}\left(k_{x}, k_{y}\right)\right| /\left|A_{x}^{n}\left(k_{x}, k_{y}\right)\right|$ in (3) is not in dBs.

${ }^{21}$ Using a PNF scan of some finite size.
} 
direction is $2 \Delta D^{d B}$. Figure 3.4-6 and Table 3.4-2 provide some useful numerical values obtained from expression (3.4.7-9).

Table 3.4-2: Error in directivity based on evaluation of (3.4.7-9), for a given fractional error in plane wave spectrum

\begin{tabular}{|c|c|}
\hline $\begin{array}{c}\text { Fractional Error in Plane } \\
\text { Wave Spectrum (dB) }\end{array}$ & $\begin{array}{c}\text { Error in } \\
\text { Directivity (dB) }\end{array}$ \\
\hline 0 & 8.7 \\
\hline-5 & 4.899 \\
\hline-10 & 2.758 \\
\hline-15 & 1.549 \\
\hline-20 & 0.87 \\
\hline-25 & 0.496 \\
\hline-30 & 0.279 \\
\hline-35 & 0.157 \\
\hline-40 & 0.087 \\
\hline-45 & 0.053 \\
\hline-50 & 0.035 \\
\hline-55 & 0.018 \\
\hline-60 & 0.009 \\
\hline
\end{tabular}




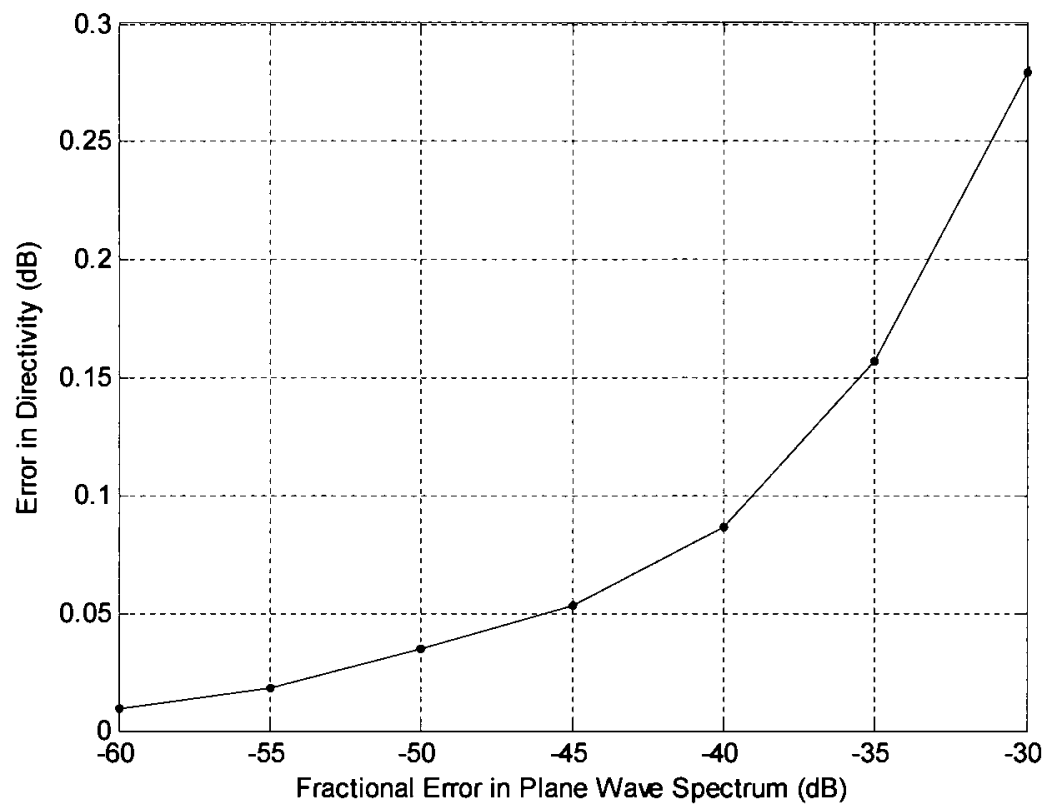

Figure 3.4-6: Error in directivity based on evaluation of 3.4.7-9, for a given fractional error in plane wave spectrum

The decision function is expressed in co-polarized and cross-polarized form as:

$$
\begin{aligned}
& F_{n}^{x}=20 \log \left\{\frac{\sum_{i=1}^{I_{\max }} \sum_{j=1}^{J_{\max }}\left\{w_{x}\left(k_{x}, k_{y}\right)\left|\frac{\left|\Delta A_{x}^{n}\left(k_{x}, k_{y}\right)\right|}{\left|A_{x}^{n}\left(k_{x}, k_{y}\right)\right|}\right|\right\}}{I_{\max } J_{\max }}\right\} \\
& F_{n}^{y}=20 \log \left\{\frac{\sum_{i=1}^{I_{\max }} \sum_{j=1}^{J_{\max }}\left\{w_{y}\left(k_{x}, k_{y}\right)\left|\frac{\left|\Delta A_{y}^{n}\left(k_{x}, k_{y}\right)\right|}{\left|A_{y}^{n}\left(k_{x}, k_{y}\right)\right|}\right|\right.}{I_{\max } J_{\max }}\right\}
\end{aligned}
$$


The weighting functions $w_{x}\left(k_{x}, k_{y}\right)$ and $w_{y}\left(k_{x}, k_{y}\right)$ are applied as follows:

$$
w_{x}\left(k_{x}, k_{y}\right)=\left\{\begin{array}{ll}
1 & \text { if } k_{x}>k_{x, \min } \text { and } k_{x}<k_{x, \max } \text { and } k_{y}>k_{y, \min } \text { and } k_{y}<k_{y, \max } \\
0 & \text { otherwise }
\end{array}\right\}
$$

$$
w_{y}\left(k_{x}, k_{y}\right)=\left\{\begin{array}{ll}
1 & \text { if } k_{x}>k_{x, \min } \text { and } k_{x}<k_{x, \max } \text { and } k_{y}>k_{y, \min } \text { and } k_{y}<k_{y, \max } \\
0 & \text { otherwise }
\end{array}\right\}
$$

The $\left(k_{x}, k_{y}\right)$ can be converted in to $\left(\theta_{i}, \phi_{j}\right)$ using expressions (2.2-20) and (2.2-21), and vice-versa.

As with the previous decision functions there is also a limiting factor for the fractional plane wave spectrum error decision functions. However, the lower limit is applied to the contour integral and the surface integral of expression (3.4.7-1), and similarly for the y-components in (3.4.7-2). This lower limit was implemented due to the pattern nulls that occur in the surface integral of the denominator of (3.4.7-1) and (3.4.72), which lead to large noise-like spikes in the resulting fractional error estimate. The lower limit is applied in the same way as in the directivity decision functions,

$$
A_{x}^{n}\left(k_{x}, k_{y}\right)=\left\{\begin{array}{cc}
A_{x}^{n}\left(k_{x}, k_{y}\right), & \text { if } A_{x}^{n}\left(k_{x}, k_{y}\right) \geq \operatorname{Max}\left(A_{x}^{n}\left(k_{x}, k_{y}\right)\right)-P_{D F N, x} \\
A_{x}^{n}\left(k_{x}, k_{y}\right)-P_{D F N, x}, & \text { otherwise }
\end{array}\right\}
$$




$$
A_{y}^{n}\left(k_{x}, k_{y}\right)=\left\{\begin{array}{cc}
A_{y}^{n}\left(k_{x}, k_{y}\right), & \text { if } A_{y}^{n}\left(k_{x}, k_{y}\right) \geq \operatorname{Max}\left(A_{y}^{n}\left(k_{x}, k_{y}\right)\right)-P_{D F N, y} \\
A_{y}^{n}\left(k_{x}, k_{y}\right)-P_{D F N, y}, & \text { otherwise }
\end{array}\right\}
$$

\subsubsection{DFN2 - Maximum of Fractional Plane Wave Spectrum Error}

DFN2 is defined much in the same way as DFE2 and DFD2,

$$
\begin{aligned}
& F_{n}^{x}=20 \log \left\{\max \left\{w_{x}\left(k_{x}, k_{y}\right)\left|\frac{\left|\Delta A_{x}^{n}\left(k_{x}, k_{y}\right)\right|}{\left|A_{x}^{n}\left(k_{x}, k_{y}\right)\right|}\right|\right\}\right\} \\
& F_{n}^{y}=20 \log \left\{\max \left\{w_{y}\left(k_{x}, k_{y}\right)\left|\frac{\left|\Delta A_{y}^{n}\left(k_{x}, k_{y}\right)\right|}{\left|A_{y}^{n}\left(k_{x}, k_{y}\right)\right|}\right|\right\}\right\}
\end{aligned}
$$

The $\mathrm{x}$ and $\mathrm{y}$ components of DFN2 are evaluated at the $\left(k_{x}, k_{y}\right)$ point at which the maximum value of $w_{x}\left(k_{x}, k_{y}\right)\left|\Delta A_{x}^{n}\right| /\left|A_{x}^{n}\right|$ and $w_{y}\left(k_{x}, k_{y}\right)\left|\Delta A_{y}^{n}\right| /\left|A_{y}^{n}\right|$ occurs, respectively. The elements of the above expressions (3.4.8-2) and (3.4.8-3) are defined as in Section 3.4.7, as is the application of the lower limits $\mathrm{P}_{\mathrm{DFN}, \mathrm{x}}$ and $\mathrm{P}_{\mathrm{DFN}, \mathrm{y}}$. 


\subsubsection{DFN3 - Fractional Plane Wave Spectrum Error at a Defined Point}

This decision function uses the same fractional plane wave spectrum error as defined in DFN1 and DFN2, except that in this case we evaluate the error estimate at some pre-defined $\left(k_{x}, k_{y}\right)$. The decision function is defined as

$$
\begin{aligned}
& F_{n}^{x}=20 \log \left\{w_{x}\left(k_{x, X}, k_{y, X}\right)\left|\frac{\left|\Delta A_{x}^{n}\left(k_{x, X}, k_{y, X}\right)\right|}{\left|A_{x}^{n}\left(k_{x, X}, k_{y, X}\right)\right|}\right|\right\} \\
& F_{n}^{y}=20 \log \left\{w_{y}\left(k_{x, Y}, k_{y, Y}\right)\left|\frac{\left|\Delta A_{y}^{n}\left(k_{x, Y}, k_{y, Y}\right)\right|}{\left|A_{y}^{n}\left(k_{x, Y}, k_{y, Y}\right)\right|}\right|\right\}
\end{aligned}
$$

where $\left(k_{x, X}, k_{y, X}\right)$ and $\left(k_{x, Y}, k_{y, Y}\right)$ are the location pre-defined $\mathrm{x}$ and $\mathrm{y}$ components, and are not necessarily the same point. The derivations of other the elements of expressions (3.4.9-1) and (3.4.9-2) follow the same procedures as DFN1 in Section 3.4.7, as is the application of the lower limits $\mathrm{P}_{\mathrm{DFN}, \mathrm{x}}$ and $\mathrm{P}_{\mathrm{DFN}, \mathrm{y}}$.

\subsection{Concluding Remarks}

The far-zone field computation time is very small compared to the near-zone data acquisition time even if such computations are done repeatedly while the data is being acquired. These computations can then be used as part of an adaptive algorithm intent on reducing the amount of data that has to be acquired by providing some feedback measure 
about the data. This has the potential to reduce the overall antenna testing time. A secondary, yet equally important result is that the feedback can be used as a type of realtime quality assessment of the near-field scan, allowing the measurement to be terminated with confidence. This work represents a first step towards building feedback/adaptivity into near-field measurements. The method has been implemented in the MATLAB-based code $A P N F$, which will be used in Chapters 4 and 5.

\subsection{References for Chapter 3}

[1] M. Dich. "Accurate determination of antenna directivity," IEEE Transactions on Antennas and Propagation, vol. 45, no. 10, pp. 1502-1505, Oct. 1997.

[2] S.F. Gregson, J. McCormic, and C. Parini. Principles of Planar Near-Field Antenna Measurements. London, UK: The Institution of Engineering and Technology, 2007, pp. 112-117.

[3] D.Garcia., "simps.m", MATLAB implementation of Simpson's numerical integration directly adapted from MATLAB function trapz, available http://www.biomecardio.com/matlab/simps.m, Aug. 2007, revised Nov. 2007.

[4] A.C.Newell, "Error analysis techniques for planar near-field measurements", IEEE Transactions on Antennas \& Propagation, vol. 36, no. 6, pp. 754-768, Jun. 1988.

[5] A.Yaghjian. Upper-Bound Errors in Far-Field Antenna Parameters Determined from Planar Near-Field Measurements - Part I, NBS Technical Note 667, USA, 1975. 


\section{CHAPTER 4 - DATA SETS USED TO EVALUATE DECISION FUNCTIONS}

\subsection{Introduction}

This chapter presents the PNF data sets that will be used to evaluate the developed decision functions. Some initial comments are given in section 4.2. Sections 4.3 through 4.7 present the characteristics of the individual antennas, and the chapter's concluding remarks are in section 4.8 .

\subsection{Preliminary Comments}

We initially considered using a theoretical analysis that allowed us to quickly compute both the near-fields and far-fields of a planar array of Hertzian dipoles. In fact such a simulator was developed. However, as the investigation progressed, it became clear that it would be more convincing to use actual experimentally acquired near-field data, measured using conventional PNF techniques. This data was chosen to be a representative set of antennas typically measured using the PNF technique. We have used such data for five different antennas. The details of the PNF data and associated antennas are provided in the sections that follow.

\subsection{Offset Reflector Antenna}

The details of the PFN scan of the offset reflector antenna can be found below in Table 4.3-1. Reflector antennas have a wide application and are typically found in radar 
and communication systems. Figure 4.3-1 and Figure 4.3-2 show the $\mathrm{E}_{\mathrm{x}}$ and $\mathrm{E}_{\mathrm{y}}$ components of the sampled PNF data. Figure 4.3-3 shows the calculated directivity of the reflector versus PNF scan size. Figure 4.3-4 through Figure 4.3-7 show the principle pattern cuts for the far-zone $\mathrm{E}_{\mathrm{co}}$ and $\mathrm{E}_{\mathrm{cr}}$ calculated using the complete PNF data set.

Table 4.3-1: PNF scan information for the offset reflector antenna

\begin{tabular}{|l|l|l|}
\hline AUT Size & Width $=0.489 \mathrm{~m}$ & Height $=0.572 \mathrm{~m}$ \\
\hline Actual PNF Scan Size & Width $=1.54 \mathrm{~m}$ & Height $=1.63 \mathrm{~m}$ \\
\hline PNF Number of Samples & $\mathrm{x}=125$ & $\mathrm{y}=133$ \\
\hline PNF Sample Spacing & $\mathrm{x}=0.0124 \mathrm{~m}$ & $\mathrm{y}=0.0124 \mathrm{~m}$ \\
\hline Probe to AUT Distance & $0.300 \mathrm{~m}$ & Probe $2=\mathrm{Ex}$ \\
\hline Frequency & $12.0 \mathrm{GHz}$ & $60^{\circ}, \mathrm{y}$ \\
\hline PNF Probe Polarization & Probe $1=\mathrm{Ey}$ & \multicolumn{2}{|l|}{} \\
\hline Rule-of-thumb Far-Field Angle & $60^{\circ}, \mathrm{x}$ & \\
\hline Calculated Directivity & $37.13 \mathrm{dBi}$ & $\mathrm{E}_{\mathrm{y}}$ \\
\hline Principle Near-Field Component &
\end{tabular}



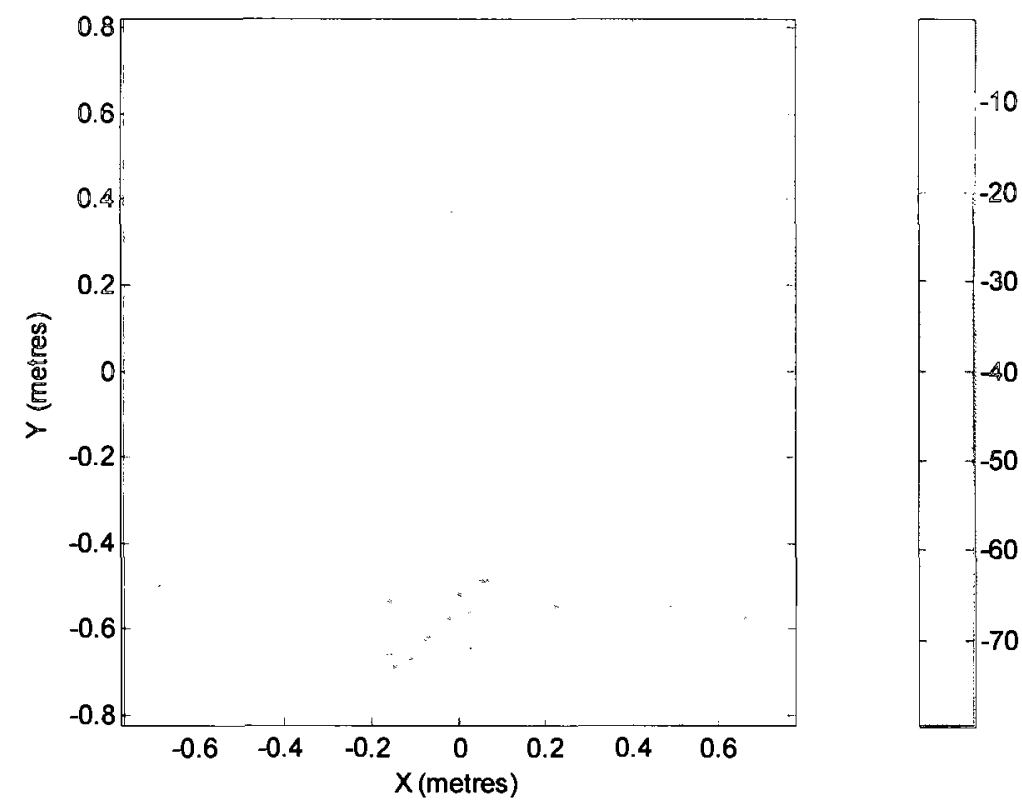

Figure 4.3-1: Near-field $\left|E_{\mathrm{x}}(x, y)\right|$ sampled over the scan plane for the offset reflector antenna, normalized to $E_{y}$, in $\mathrm{dB}$
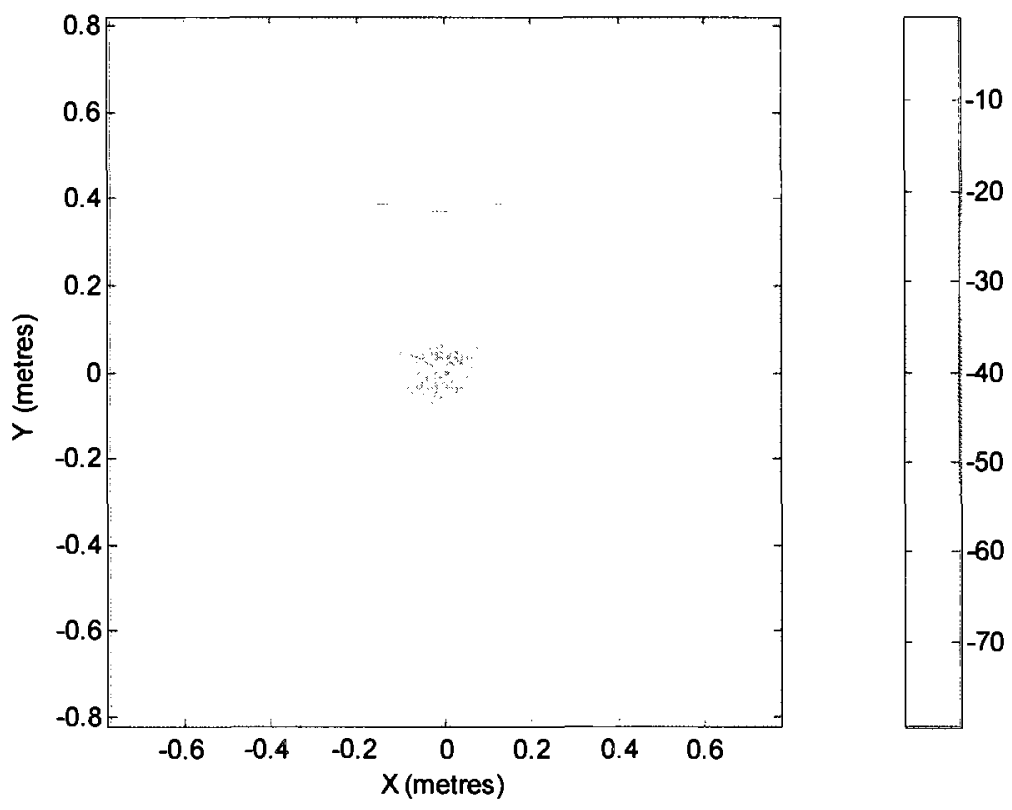

Figure 4.3-2: Normalised near-field $\left|E_{y}(x, y)\right|$ sampled over the scan plane for the offset reflector antenna, in $\mathrm{dB}$ 


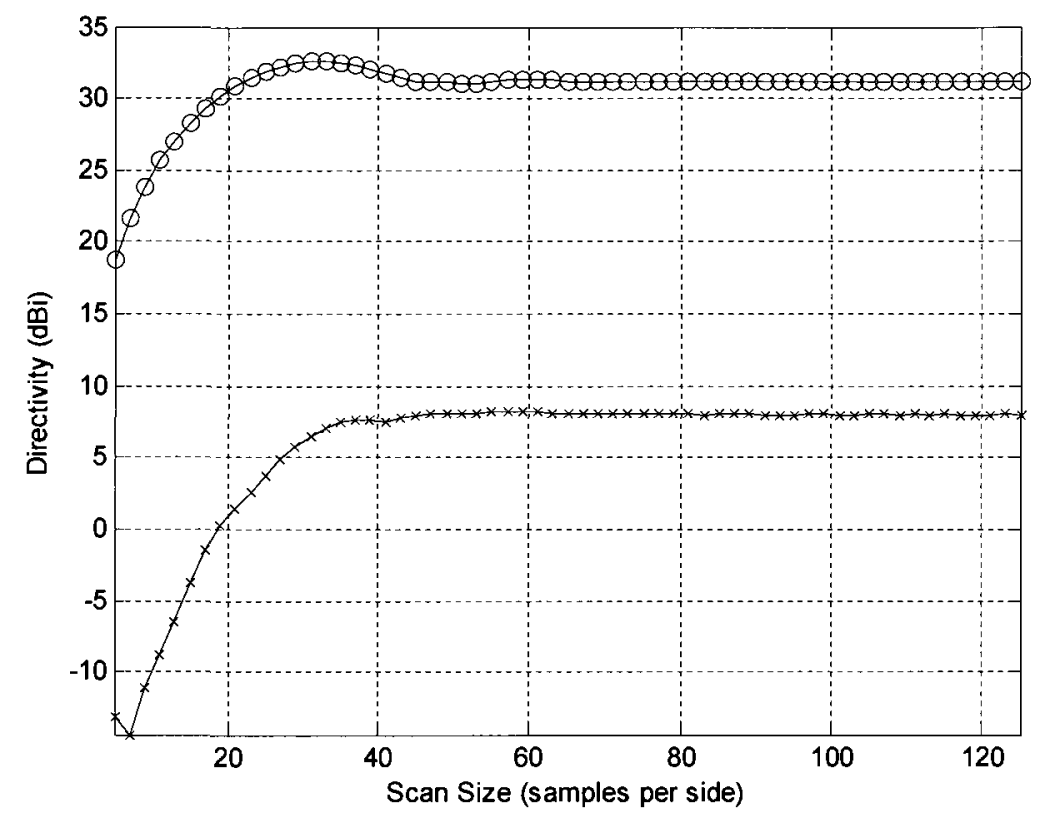

Figure 4.3-3: Co (o) and cross ( $x$ ) polarized directivity of the offset reflector antenna based on near-field scan size

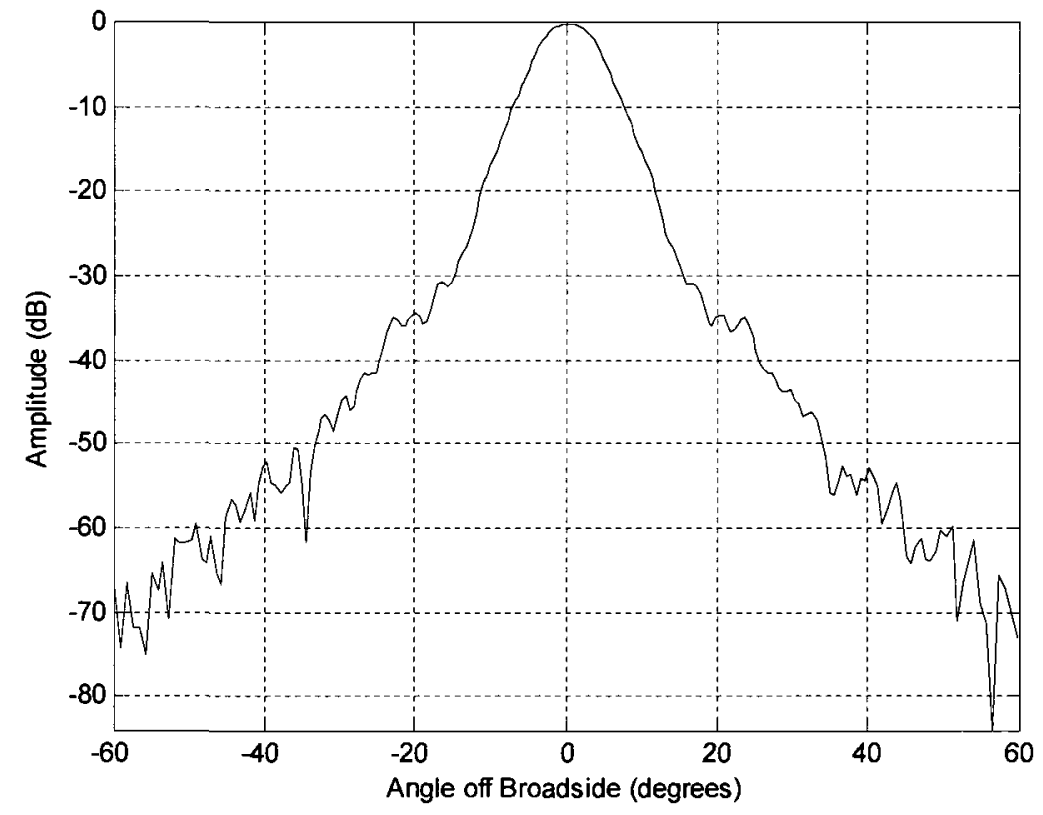

Figure 4.3-4: Normalized far-field $\left|E_{\mathrm{co}}(\theta, \phi)\right|$ of the offset reflector antenna for pattern cut $\phi=0^{\circ}$ 


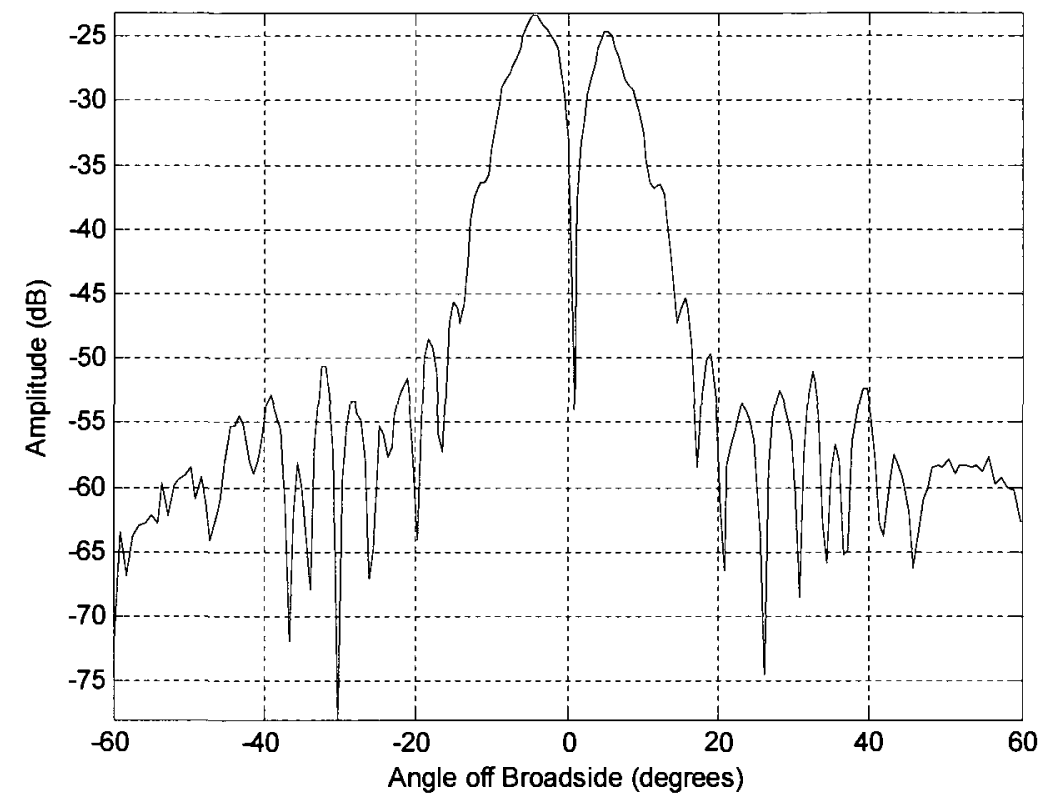

Figure 4.3-5: Far-field $\left|\boldsymbol{E}_{\mathrm{cr}}(\theta, \phi)\right|$ of the offset reflector antenna for pattern cut $\phi=$ $0^{\circ}$, normalized to $\left|E_{\mathrm{co}}(\theta, \phi)\right|$

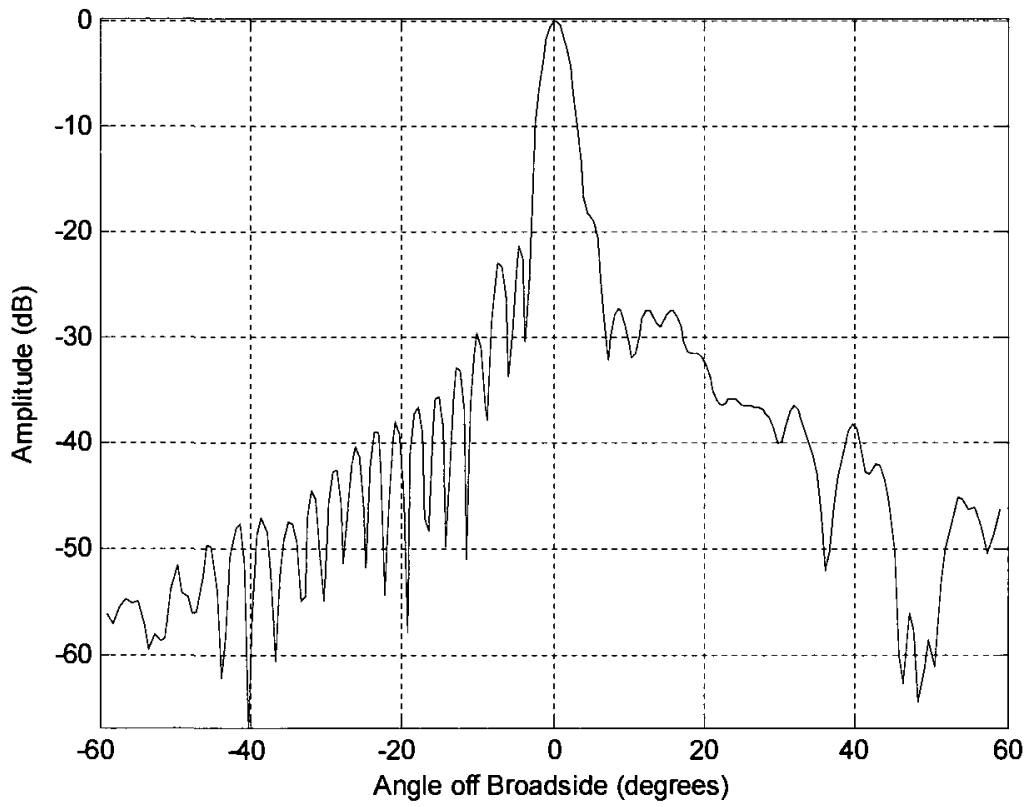

Figure 4.3-6: Normalized far-field $\left|E_{\mathrm{co}}(\theta, \phi)\right|$ of the offset reflector antenna for pattern cut $\phi=90^{\circ}$ 


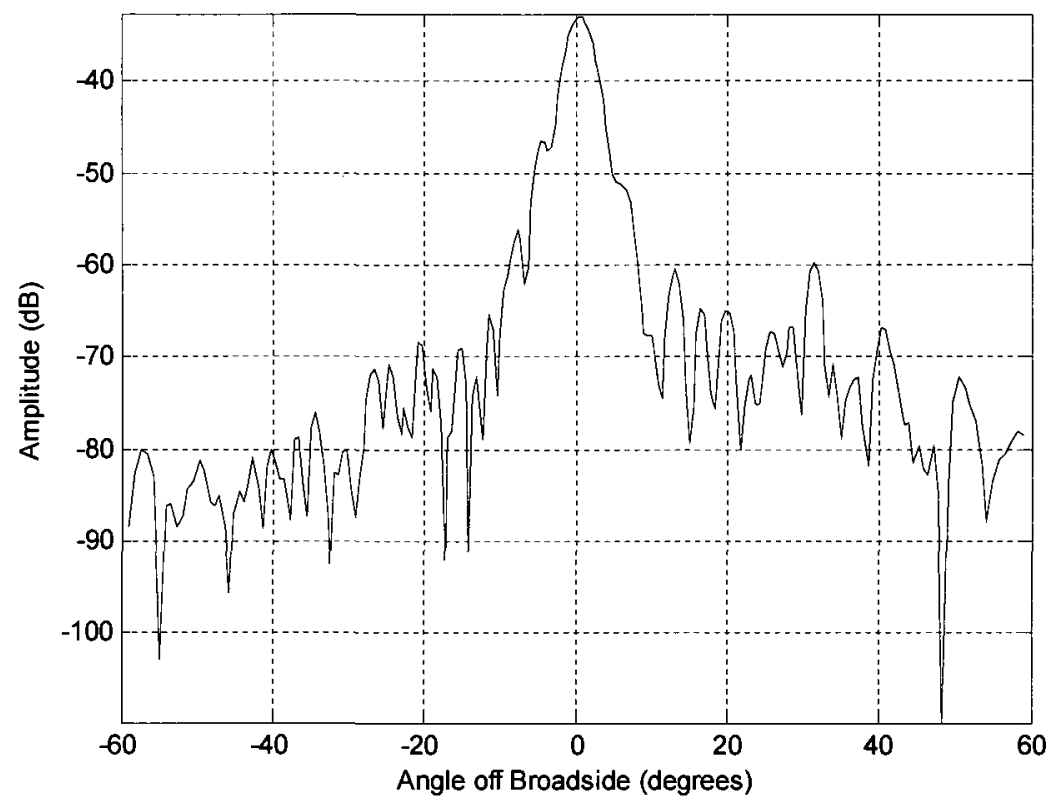

Figure 4.3-7: Far-field $\left|E_{\mathrm{cr}}(\theta, \phi)\right|$ of the offset reflector antenna for pattern cut $\phi=$ $90^{\circ}$, normalized to $\left|E_{\mathrm{co}}(\theta, \phi)\right|$

Figure 4.3-8 shows the rule-of-thumb valid far-zone angle off broadside based on the PNF scan size (in samples per side) for the offset reflector antenna. The largest aperture dimension of the AUT is represented by the black vertical line. 


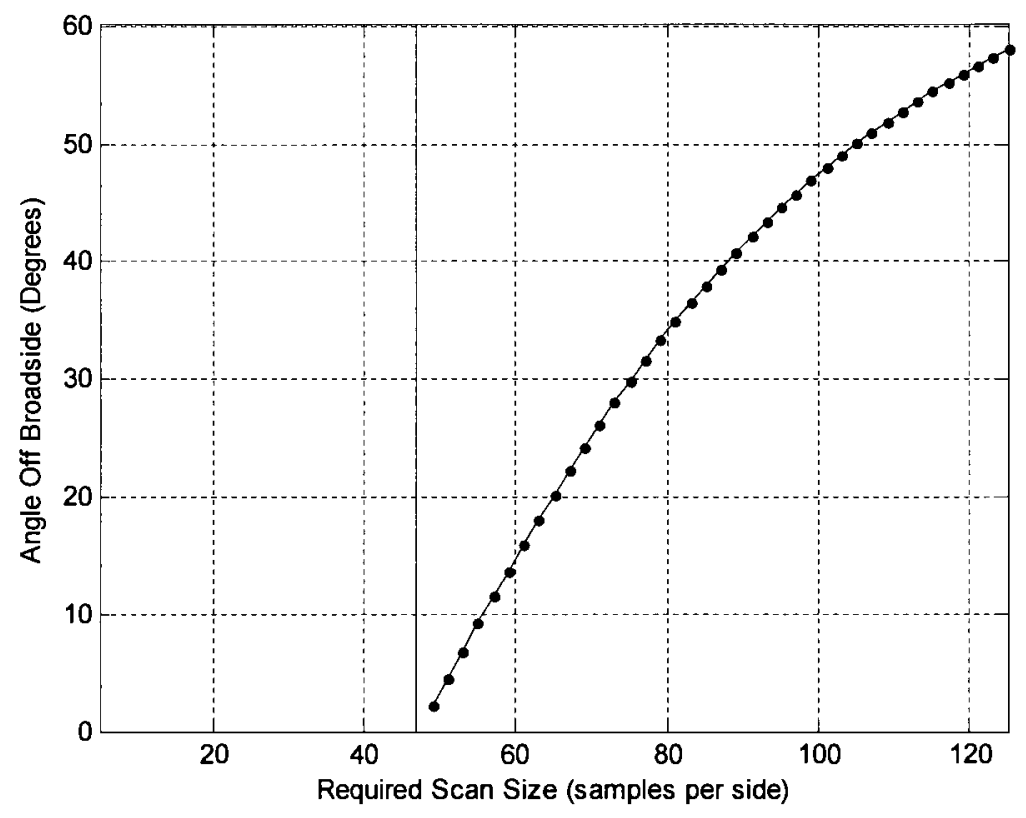

Figure 4.3-8: Rule-of-thumb valid far-zone angle off broadside for the offset reflector antenna. The vertical line shows the AUT aperture

\subsection{Horn Antenna}

Details about the PNF scan of the horn antenna can be found below in Table 4.4-1. Horn antennas are commonly used as the active element in dish antennas. Figure 4.4-1 and Figure 4.4-2 show the $\mathrm{x}$ and $\mathrm{y}$ components of the E-fields from the horn PNF data set. Figure 4.4-3 shows the directivity of the horn antenna as a function of scan size. Figure 4.4-4 through Figure 4.4-7 show the principle pattern cuts for the far-zone $\mathrm{E}_{\mathrm{co}}$ and $\mathrm{E}_{\mathrm{cr}}$, using the complete PNF data set. 
Table 4.4-1: PNF scan information for the horn antenna

\begin{tabular}{|l|l|l|}
\hline AUT Size & Width $=1.2 \mathrm{~m}$ & Height $=1.2 \mathrm{~m}$ \\
\hline Actual PNF Scan Size & Width $=3.0 \mathrm{~m}$ & Height $=3.0 \mathrm{~m}$ \\
\hline PNF Number of Samples & $\mathrm{x}=83$ & $\mathrm{y}=83$ \\
\hline PNF Sample Spacing & $\mathrm{x}=0.0366 \mathrm{~m}$ & $\mathrm{y}=0.0366 \mathrm{~m}$ \\
\hline Probe to AUT Distance & $1.385 \mathrm{~m}$ & \multicolumn{2}{|l}{} \\
\hline Frequency & $4.0 \mathrm{GHz}$ & Probe $2=\mathrm{Ey}$ \\
\hline PNF Probe Polarization & Probe $1=\mathrm{Ex}$ & $60^{\circ}, \mathrm{y}$ \\
\hline Rule-of-thumb Far-Field Angle & $60^{\circ}, \mathrm{x}$ \\
\hline Calculated Directivity & $21.1 \mathrm{dBi}$ \\
\hline Principle Near-Field Component & $\mathrm{E}_{\mathrm{x}}$ \\
\hline
\end{tabular}

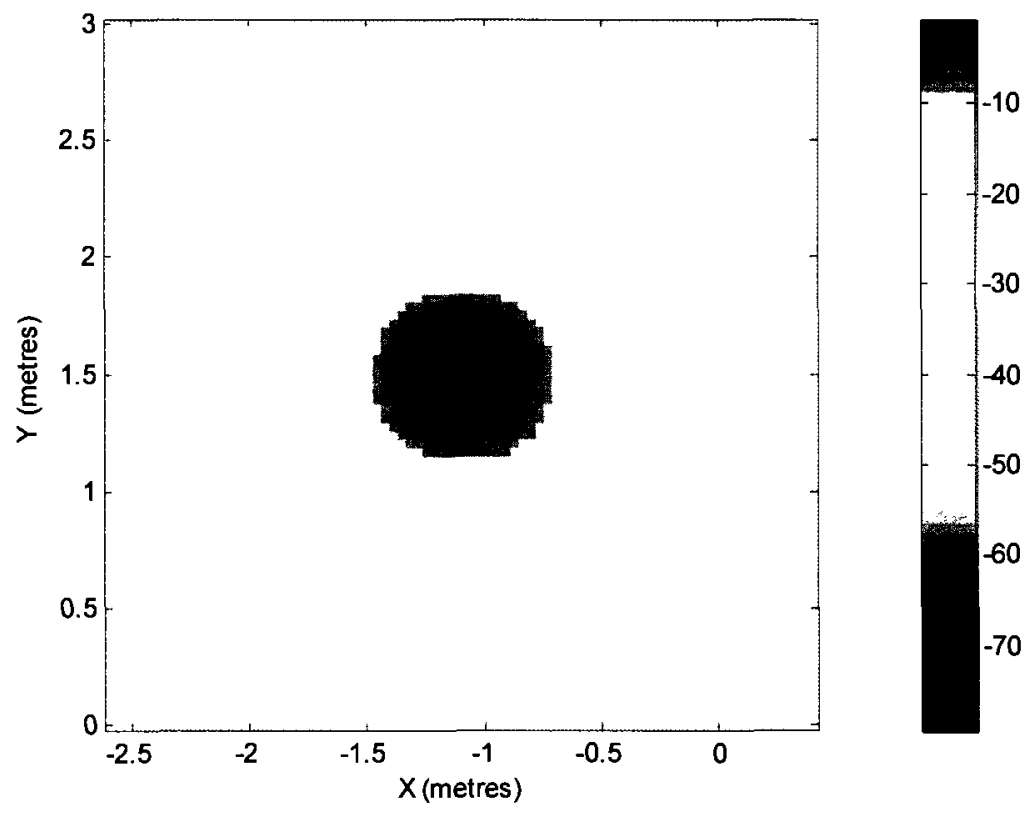

Figure 4.4-1: Normalized near-field $\left|E_{\mathbf{x}}(x, y)\right|$ sampled over the scan plane for the horn antenna, in $\mathrm{dB}$ 


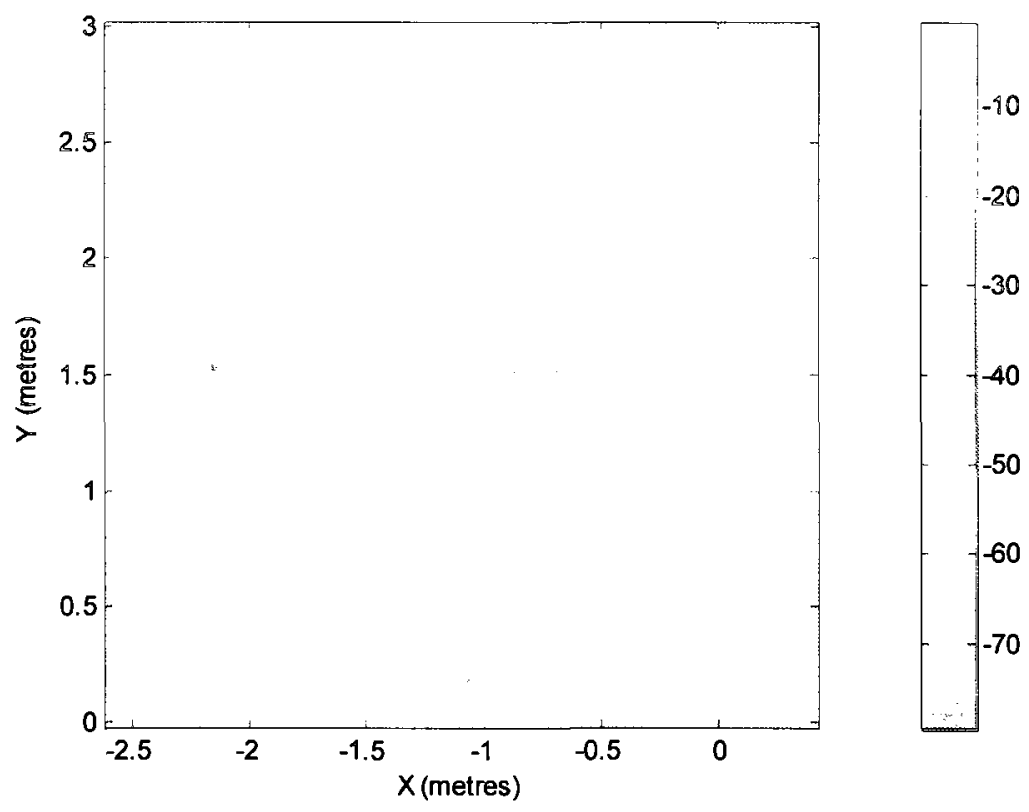

Figure 4.4-2: Near-field $\left|E_{\mathrm{y}}(x, y)\right|$ sampled over the scan plane for the horn antenna, normalized to $E_{\mathrm{x}}$, in $\mathrm{dB}$

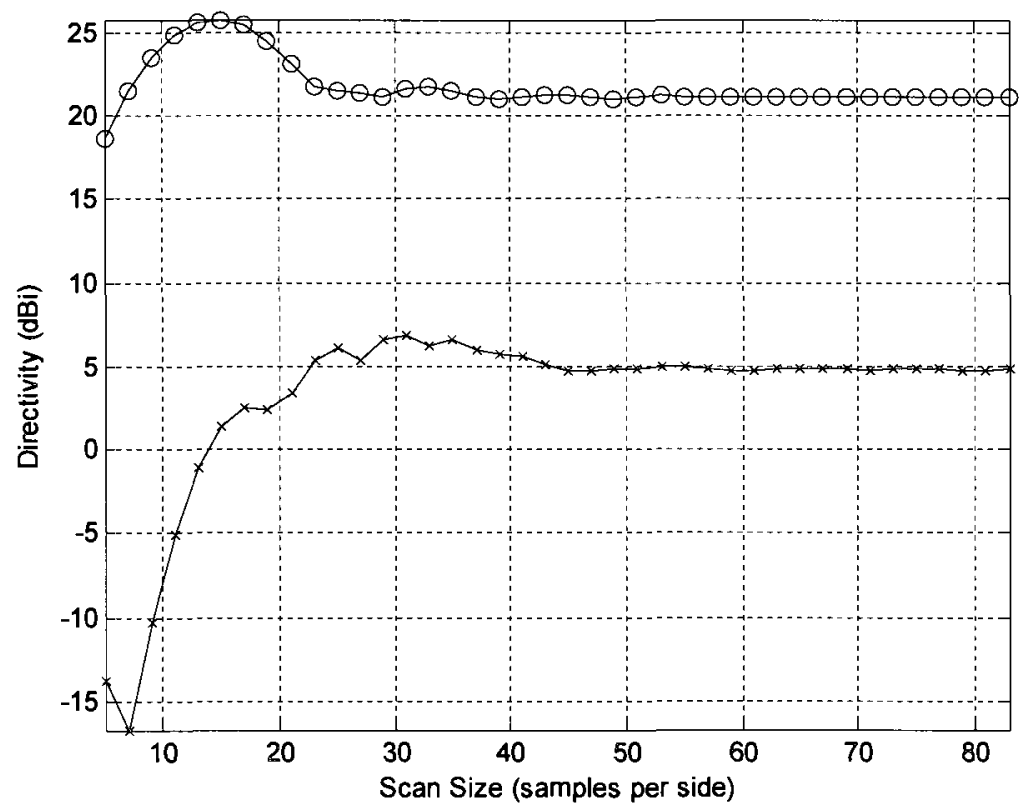

Figure 4.4-3: $\mathrm{Co}(0)$ and cross (x) polarized directivity of the horn antenna based on near-field scan size 


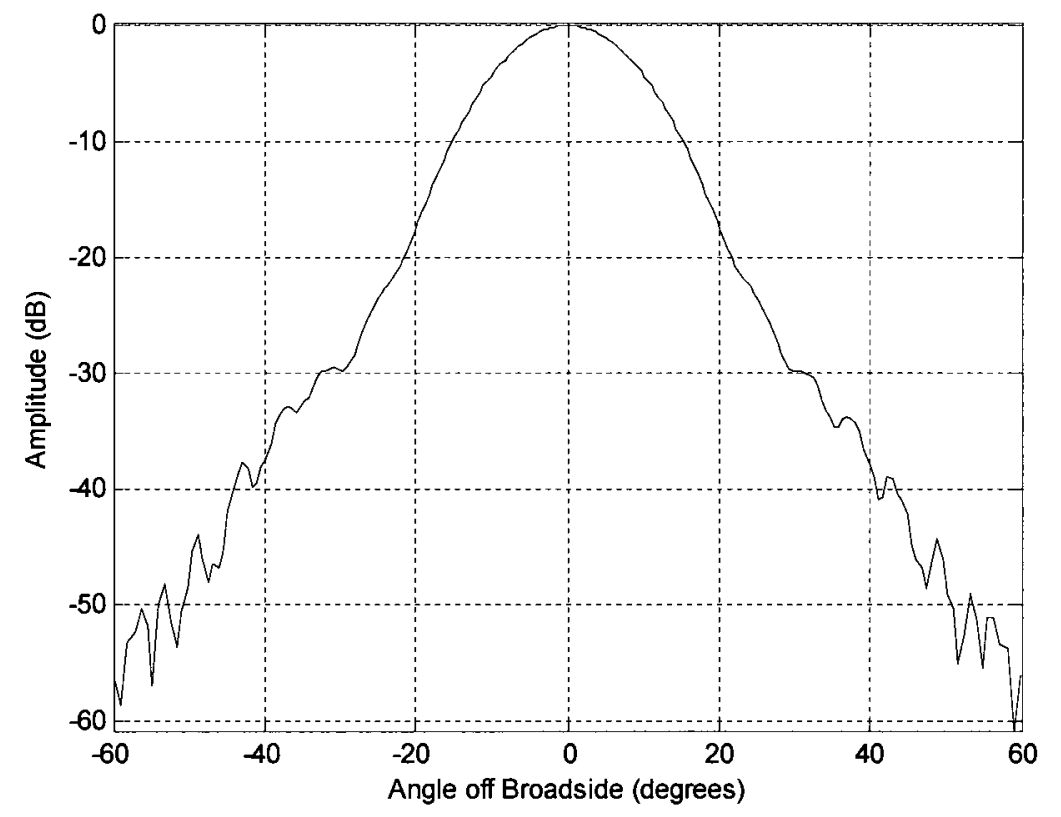

Figure 4.4-4: Normalized far-field $\left|E_{\mathrm{co}}(\theta, \phi)\right|$ of the horn antenna for pattern cut $\phi=$ $0^{\circ}$

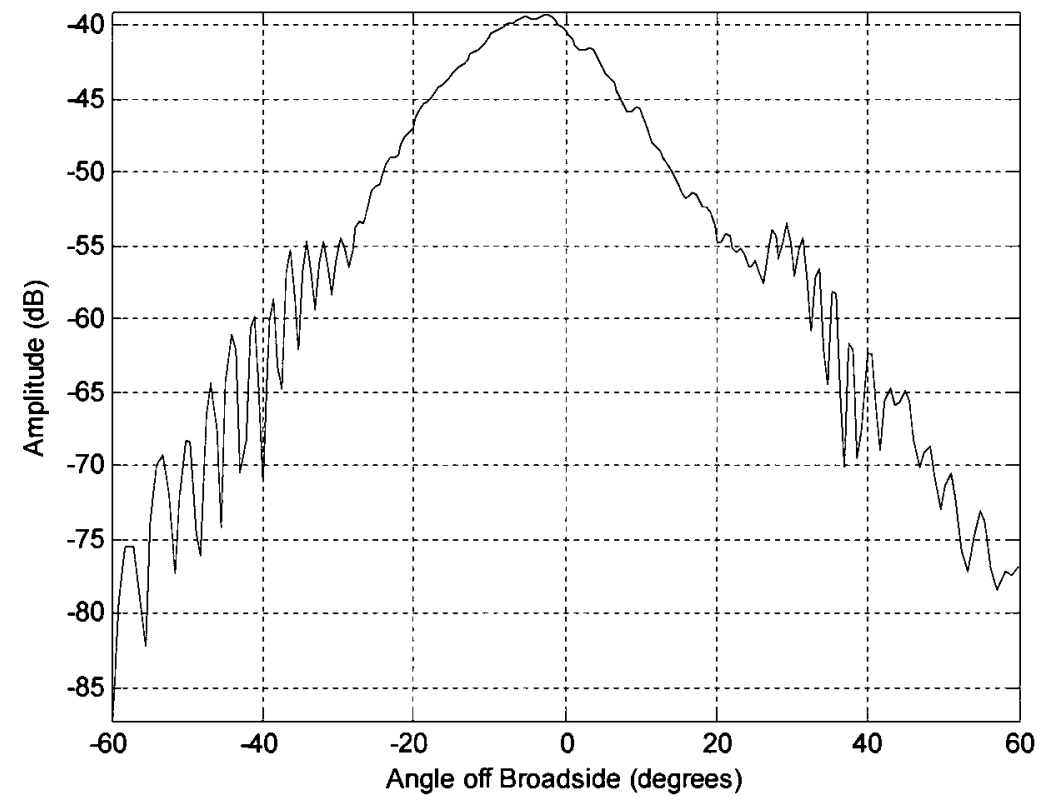

Figure 4.4-5: Far-field $\left|E_{\mathrm{cr}}(\theta, \phi)\right|$ of the horn antenna for pattern cut $\phi=0^{\circ}$, normalized to $\left|E_{\mathrm{co}}(\theta, \phi)\right|$ 


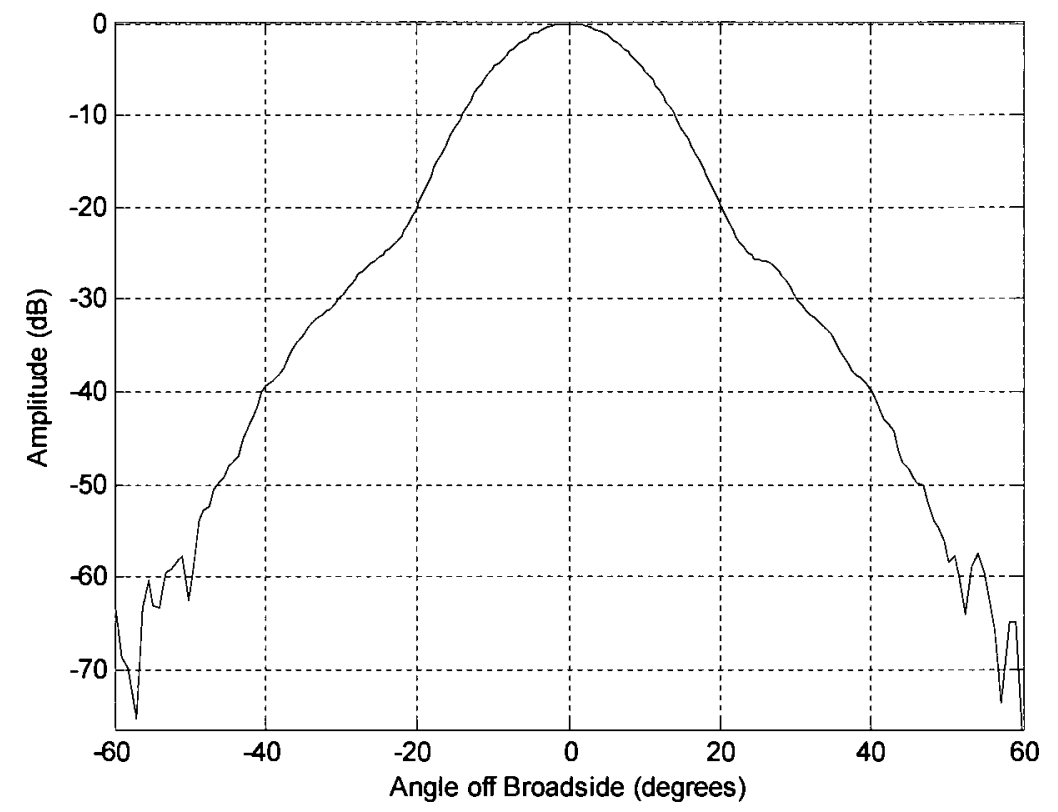

Figure 4.4-6: Normalized far-field $\left|E_{\mathrm{co}}(\theta, \phi)\right|$ of the horn antenna for the pattern $\operatorname{cut} \phi=90^{\circ}$

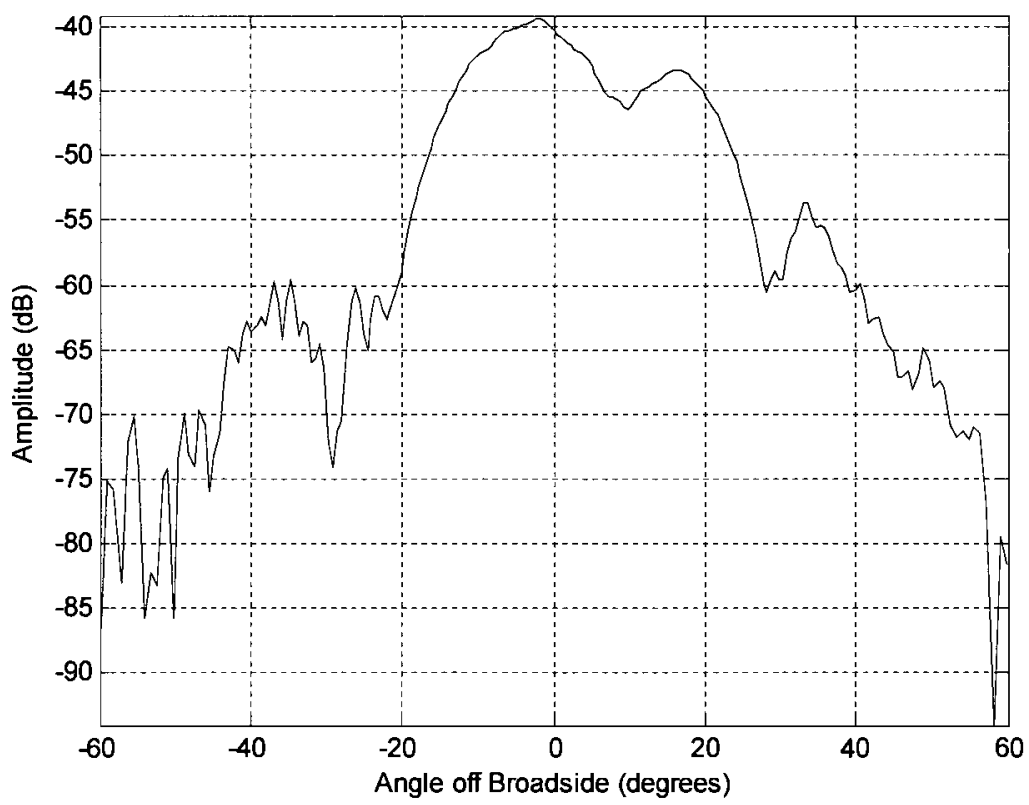

Figure 4.4-7: Far-field $\left|E_{\mathrm{cr}}(\theta, \phi)\right|$ of the horn antenna for pattern cut $\phi=90^{\circ}$, normalized to $\left|E_{\mathrm{co}}(\theta, \phi)\right|$ 
Figure 4.4-8 shows the rule-of-thumb valid far-zone angle off broadside based on the PNF scan size (in samples per side) for the horn antenna. The largest aperture dimension of the AUT is represented by the black vertical line.

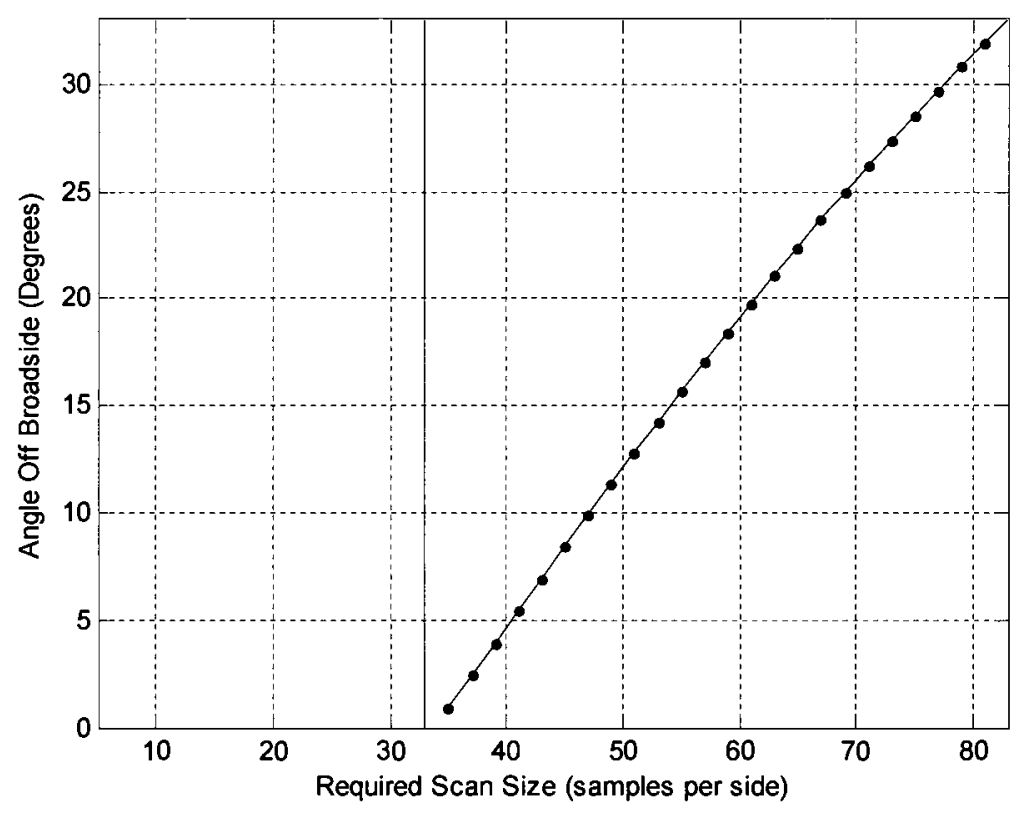

Figure 4.4-8: Rule-of-thumb valid far-zone angle off broadside for the horn antenna. The vertical line shows the AUT aperture

\subsection{Planar Array}

The details of the PNF scan of the planar array can be found below in Table 4.5-1. Figure 4.5-1 and Figure 4.5-2 show the $\mathrm{x}$ and y component of the sampled PNF E-field data for the planar array. Figure 4.5-3 shows the calculated directivity as a function of PNF scan size. Figure 4.5-4 through Figure 4.5-7 show the principle cuts of the far-zone $E_{c o}$ and $E_{c r}$ based on the complete PNF data set. Based on the characteristics of the 
planar array PNF data set the maximum far-zone region of validity based on the rule-ofthumb calculation for this antenna is only $20^{\circ}$ off broadside.

Table 4.5-1: PNF scan information for the planar array

\begin{tabular}{|l|l|l|}
\hline AUT Size & Width $=1.02 \mathrm{~m}$ & Height $=1.02 \mathrm{~m}$ \\
\hline Actual PNF Scan Size & Width $=1.43 \mathrm{~m}$ & Height $=1.43 \mathrm{~m}$ \\
\hline PNF Number of Samples & $\mathrm{x}=105$ & $\mathrm{y}=105$ \\
\hline PNF Sample Spacing & $\mathrm{x}=0.0137 \mathrm{~m}$ & $\mathrm{y}=0.0137 \mathrm{~m}$ \\
\hline Probe to AUT Distance & $0.533 \mathrm{~m}$ & Probe $2=\mathrm{Ex}$ \\
\hline Frequency & $10.25 \mathrm{GHz}$ & $20^{\circ}, \mathrm{y}$ \\
\hline PNF Probe Polarization & Probe $1=\mathrm{Ey}$ \\
\hline Rule-of-thumb Far-Field Angle & $20^{\circ}, \mathrm{x}$ & \multicolumn{2}{|l}{} \\
\hline Calculated Directivity & $34.15 \mathrm{dBi}$ \\
\hline Principle Near-Field Component & $\mathrm{E}_{\mathrm{y}}$ \\
\hline
\end{tabular}




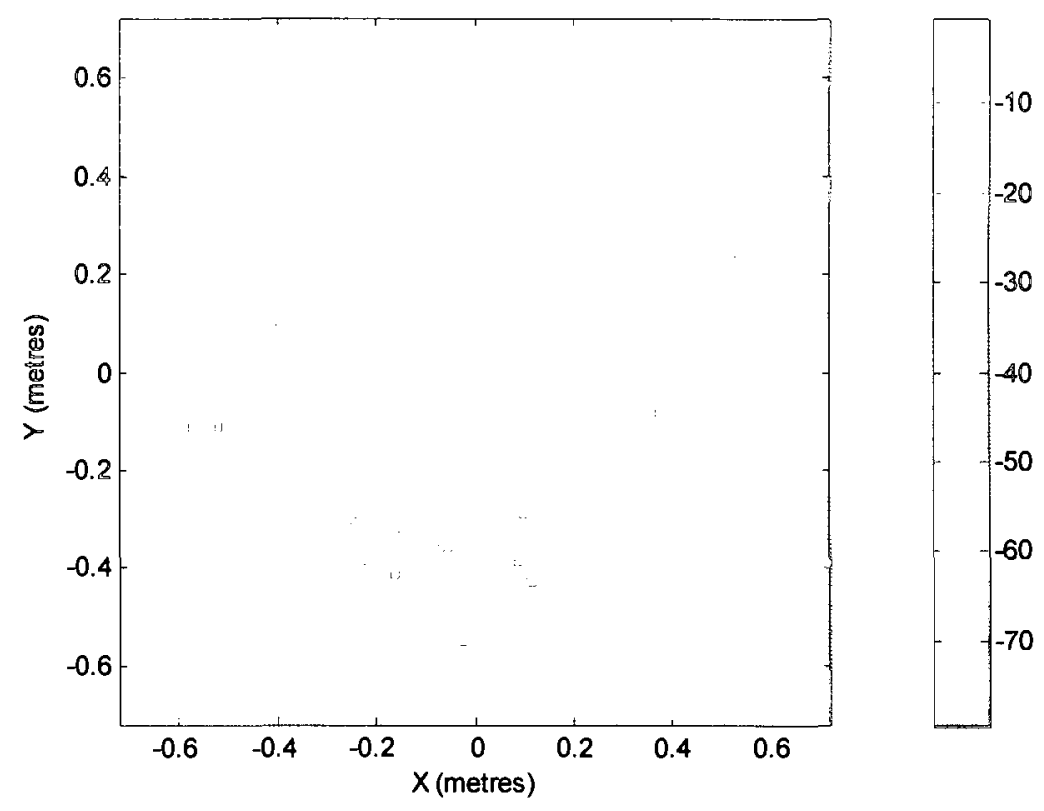

Figure 4.5-1: Near-field $\left|E_{\mathrm{x}}(x, y)\right|$ sampled over the scan plane for the planar array, normalized to $E_{\mathrm{y}}$, in $\mathrm{dB}$

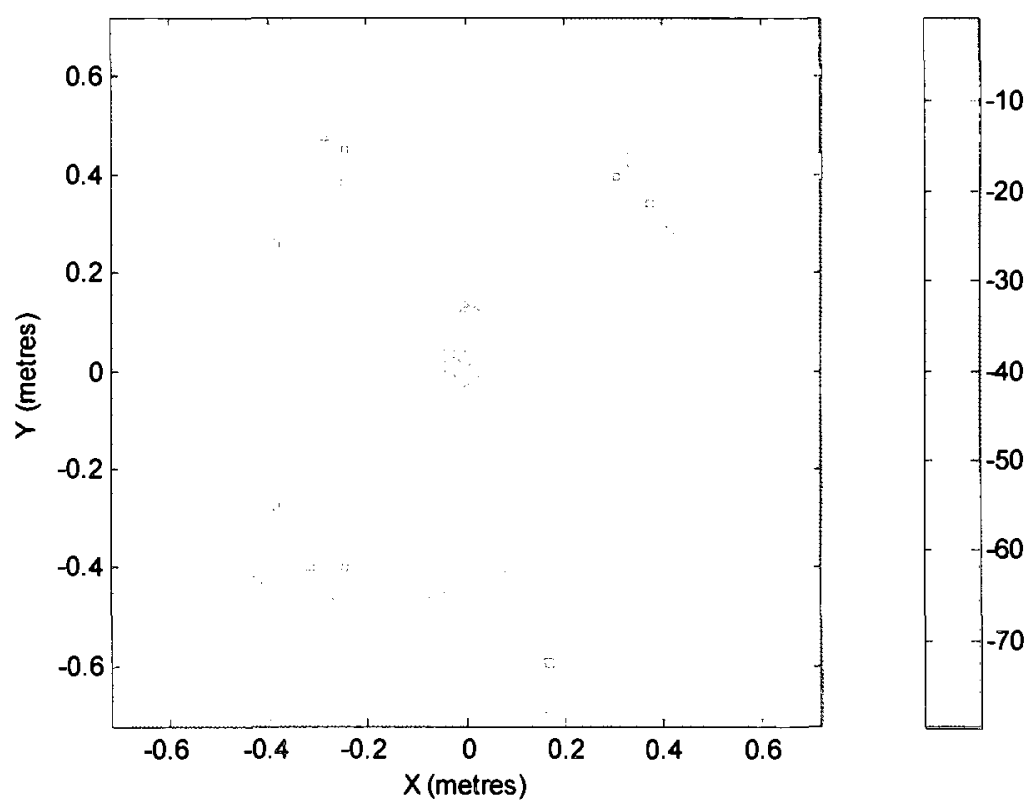

Figure 4.5-2: Normalized near-field $\left|E_{\mathrm{y}}(x, y)\right|$ sampled over the scan plane for the planar array, in dB 


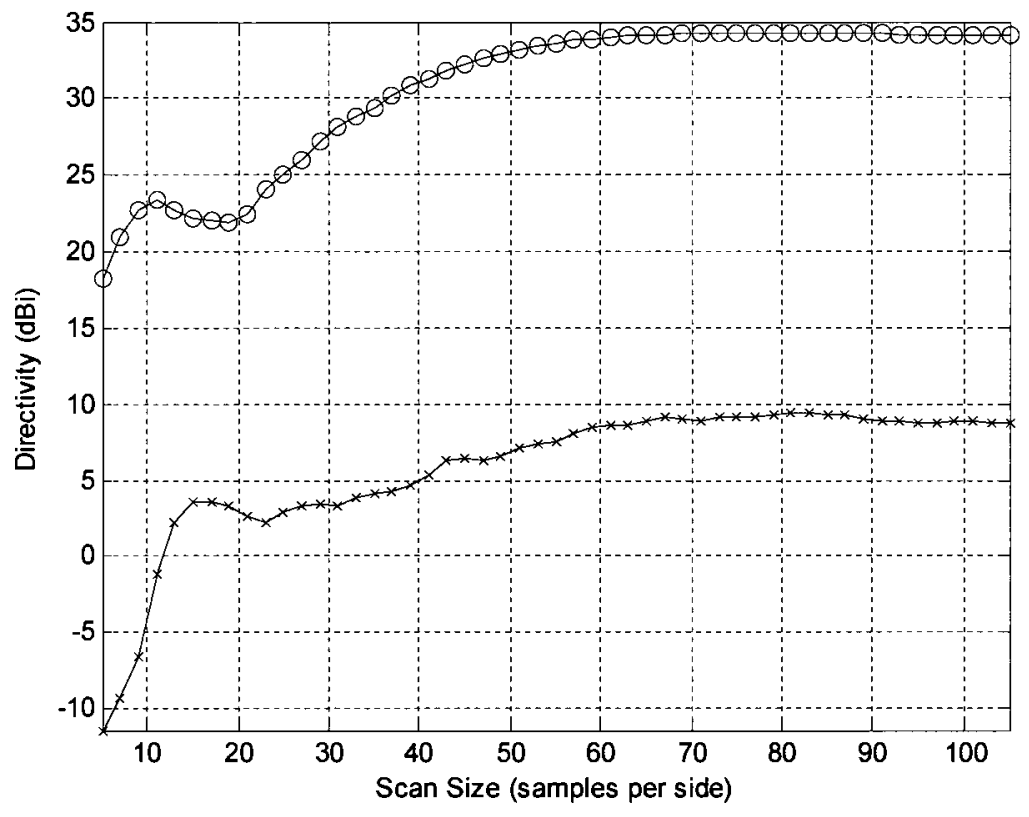

Figure 4.5-3: Co (o) and cross (x) polarized directivity of planar array based on near-field scan size

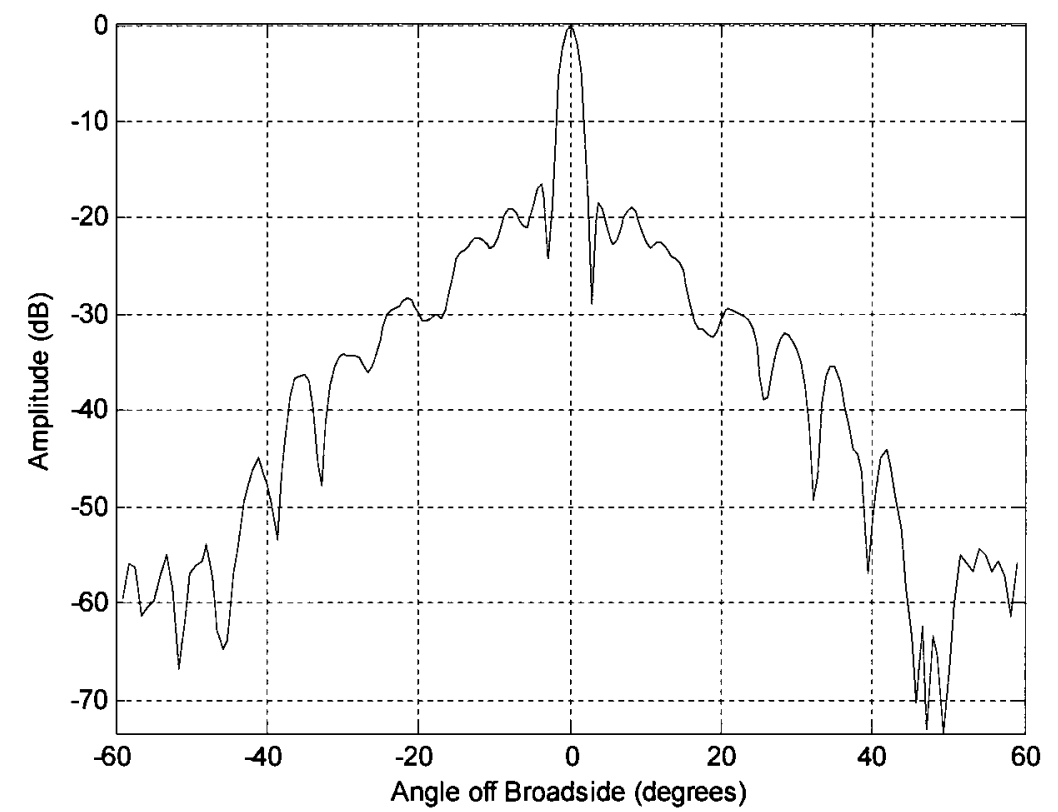

Figure 4.5-4: Normalized far-field $\left|E_{\mathrm{co}}(\theta, \phi)\right|$ of the planar array for pattern cut $\phi=$ $0^{\circ}$ 


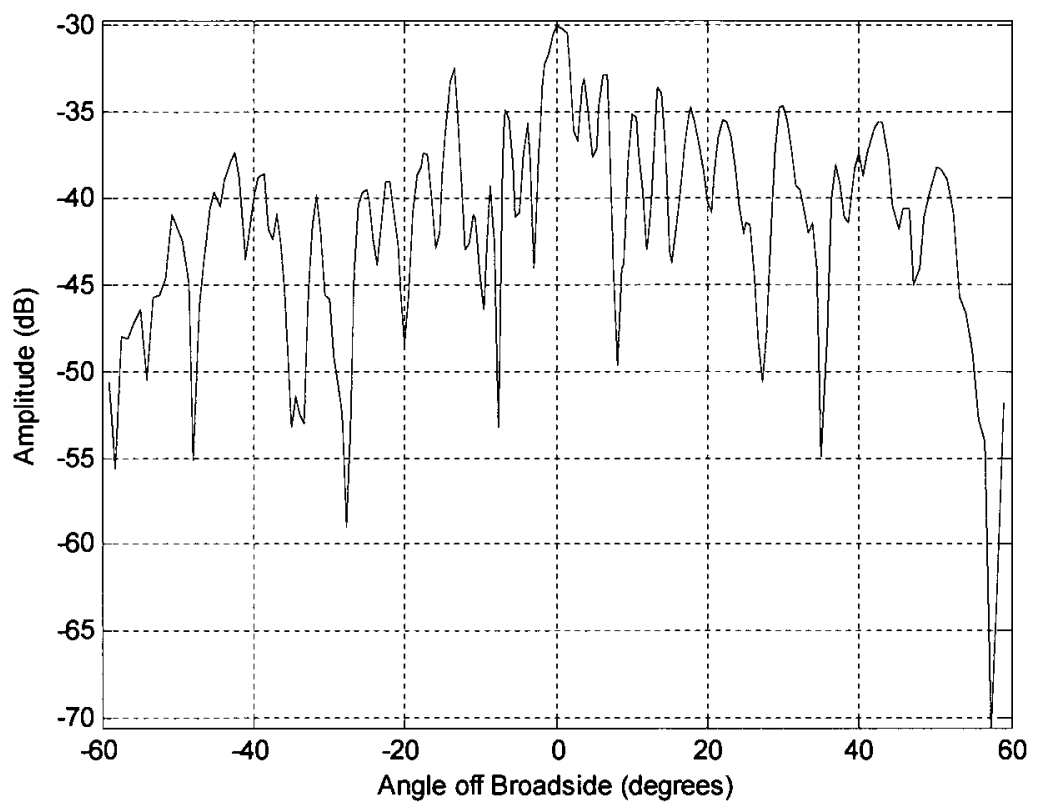

Figure 4.5-5: Far-field $\left|E_{\mathrm{cr}}(\theta, \phi)\right|$ of the planar array for pattern cut $\phi=0^{\circ}$, normalized to $\left|E_{\mathrm{co}}(\theta, \phi)\right|$

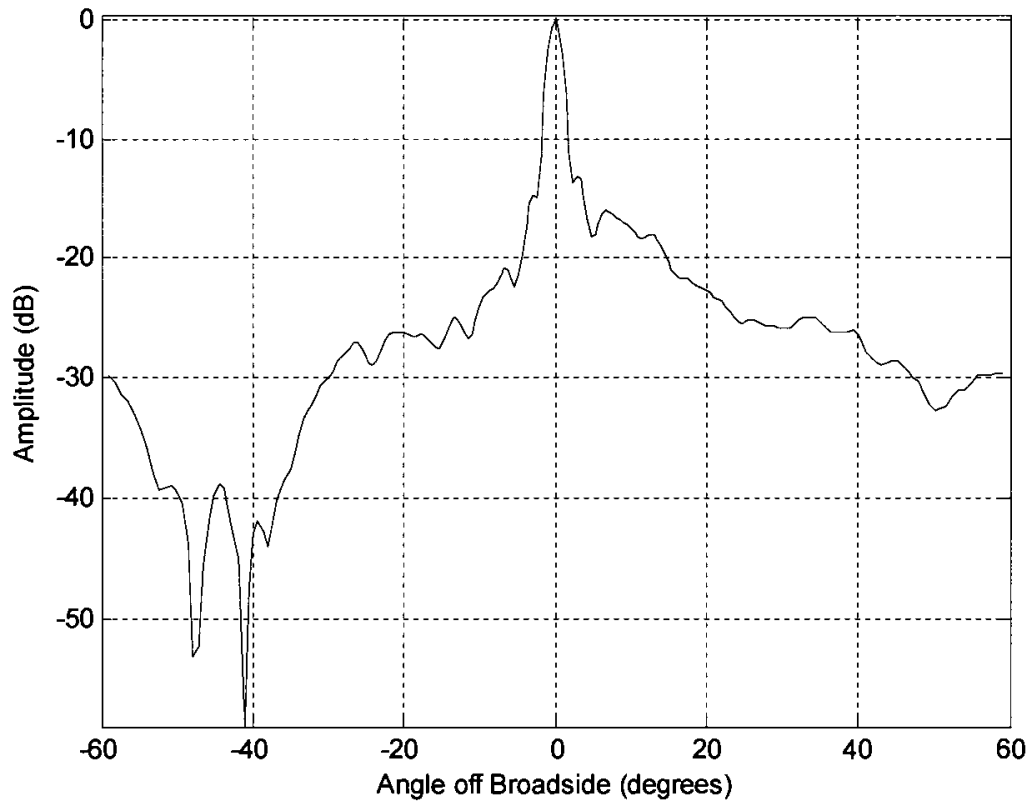

Figure 4.5-6: Normalized far-field $\left|E_{\mathrm{co}}(\theta, \phi)\right|$ of the planar array for pattern cut $\phi=$ $90^{\circ}$ 


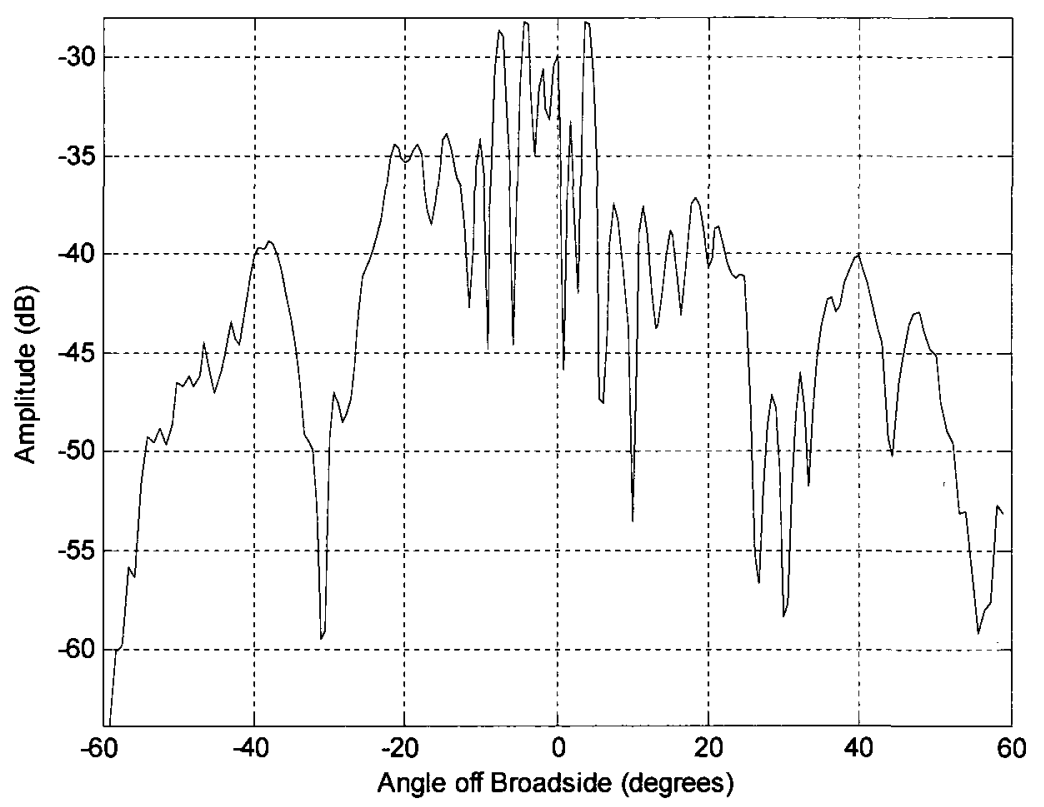

Figure 4.5-7: Far-field $\left|E_{\mathrm{cr}}(\theta, \phi)\right|$ of the planar array for pattern cut $\phi=90^{\circ}$, normalized to $\left|E_{\mathrm{co}}(\theta, \phi)\right|$

Figure 4.5-8 shows the rule-of-thumb valid far-zone angle off broadside based on the PNF scan size (in samples per side) for the planar array. The largest aperture dimension of the AUT is represented by the black vertical line. As indicated by the ruleof-thumb calculation the planar array has the smallest far-zone region of validity of all the antennas investigated in this thesis. 


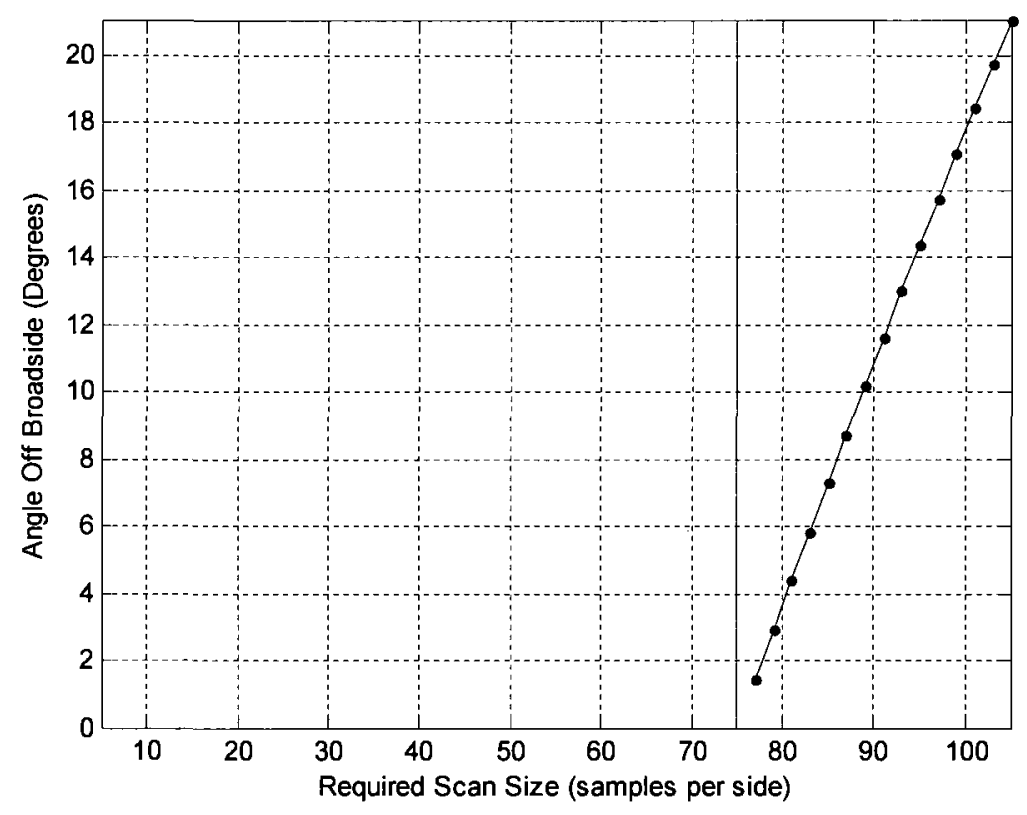

Figure 4.5-8: Rule-of-thumb valid far-zone angle off broadside for the planar array. The vertical line shows the AUT aperture

\subsection{Monopulse Reflector Antenna}

The details of the PNF scan of the monopulse reflector antenna can be found below in Table 4.6-1. Monopulse antennas are typically used in tracking systems. Figure 4.6-1 and Figure 4.6-2 show the $\mathrm{x}$ and $\mathrm{y}$ component of the sampled PNF E-field data. Figure 4.6-3 shows the calculated directivity of the monopulse reflector antenna based on PNF scan size. Figure 4.6-4 through Figure 4.6-7 show the principle pattern cuts for the far-zone co- and cross-polarized E-fields, based on the complete PNF data set. 
Table 4.6-1: PNF scan information for the monopulse reflector antenna

\begin{tabular}{|l|l|l|}
\hline AUT Size & Width $=0.508 \mathrm{~m}$ & Height $=1.02 \mathrm{~m}$ \\
\hline Actual PNF Scan Size & Width $=1.52 \mathrm{~m}$ & Height $=2.03 \mathrm{~m}$ \\
\hline PNF Number of Samples & $\mathrm{x}=150$ & $\mathrm{y}=198$ \\
\hline PNF Sample Spacing & $\mathrm{x}=0.0102 \mathrm{~m}$ & $\mathrm{y}=0.0103 \mathrm{~m}$ \\
\hline Probe to AUT Distance & $0.127 \mathrm{~m}$ & Probe $2=\mathrm{Ex}$ \\
\hline Frequency & $14.00 \mathrm{GHz}$ & $60^{\circ}, \mathrm{y}$ \\
\hline PNF Probe Polarization & Probe $1=\mathrm{Ey}$ & \multicolumn{2}{|l}{} \\
\hline Rule-of-thumb Far-Field Angle & $60^{\circ}, \mathrm{x}$ & \\
\hline Calculated Directivity & $38.21 \mathrm{dBi}$ \\
\hline Principle Near-Field Component & $\mathrm{E}_{\mathrm{y}}$ &
\end{tabular}

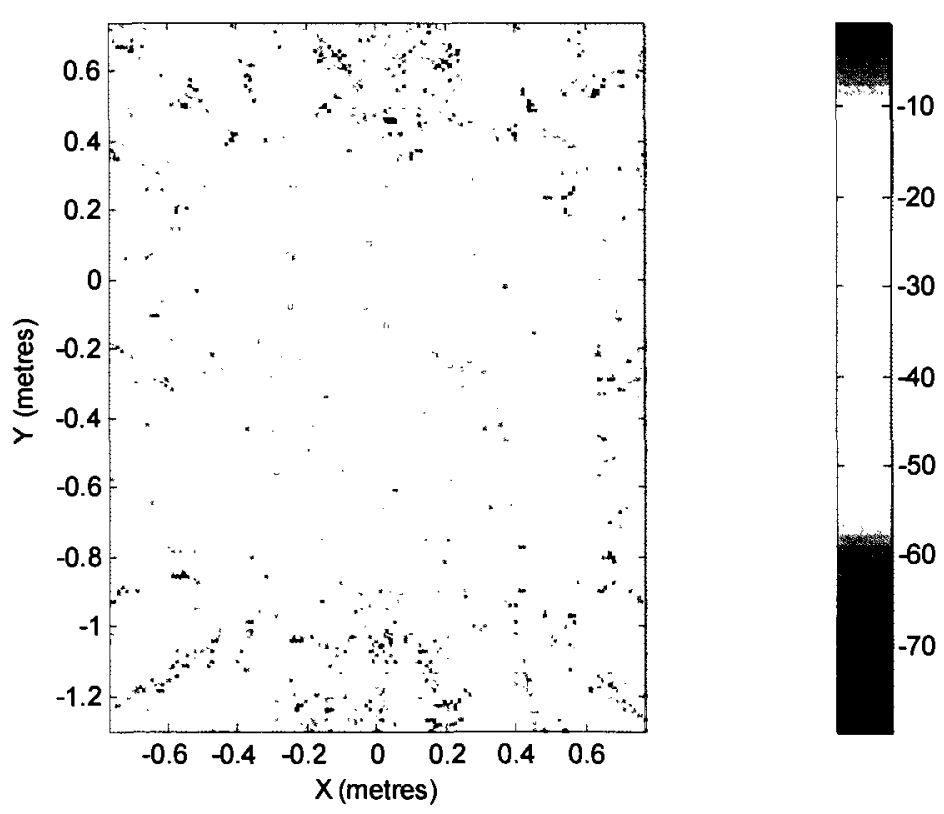

Figure 4.6-1: Near-field $\left|E_{\mathrm{x}}(x, y)\right|$ sampled over the scan plane for the monopulse reflector antenna, normalized to $E_{\mathrm{y}}$, in $\mathrm{dB}$ 

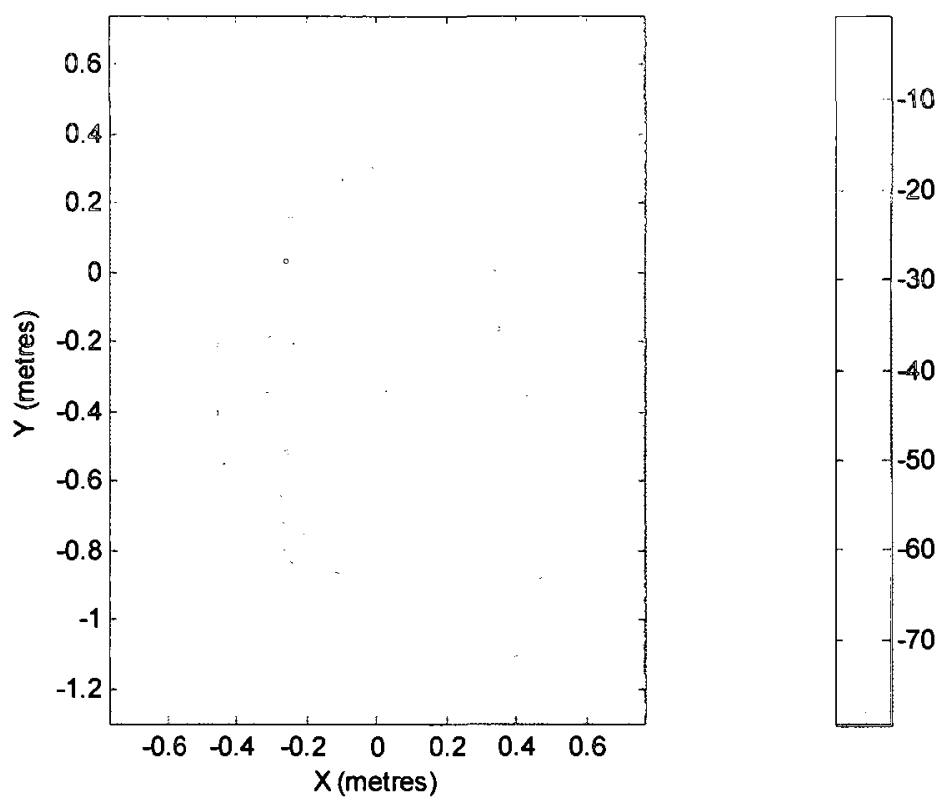

Figure 4.6-2: Normalized near-field $\left|E_{y}(x, y)\right|$ sampled over the scan plane for the monopulse reflector antenna, in $\mathbf{d B}$

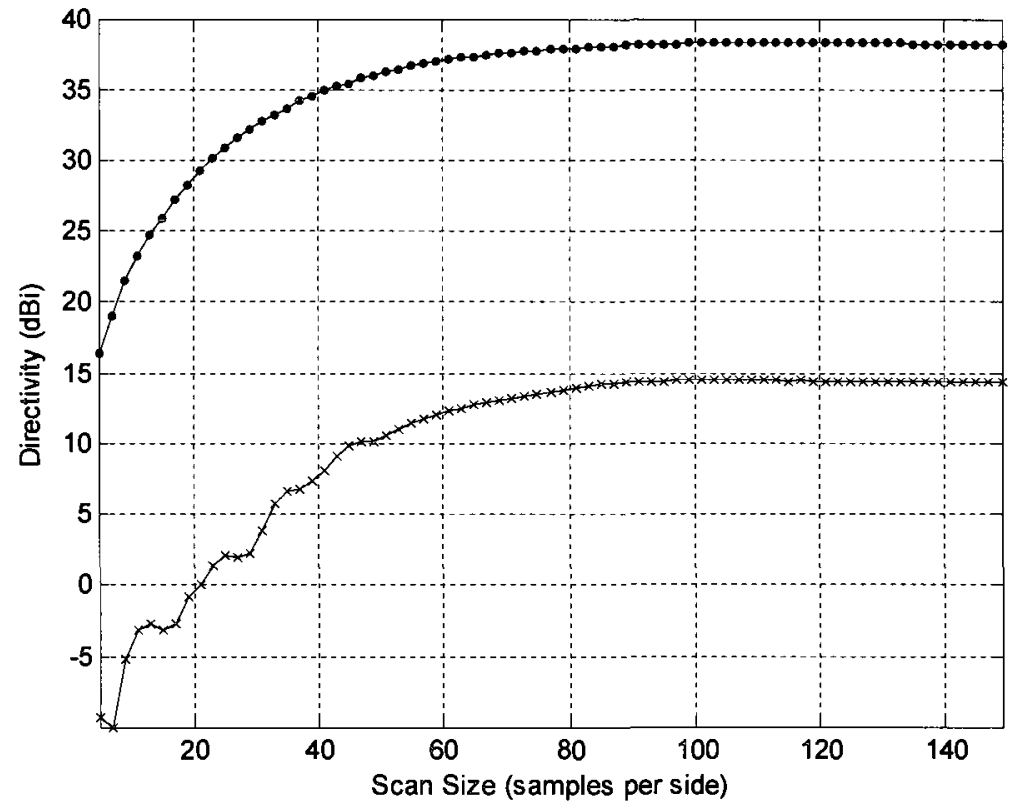

Figure 4.6-3: Co (o) and cross (x) polarized directivity of monopulse reflector antenna based on near-field scan size 


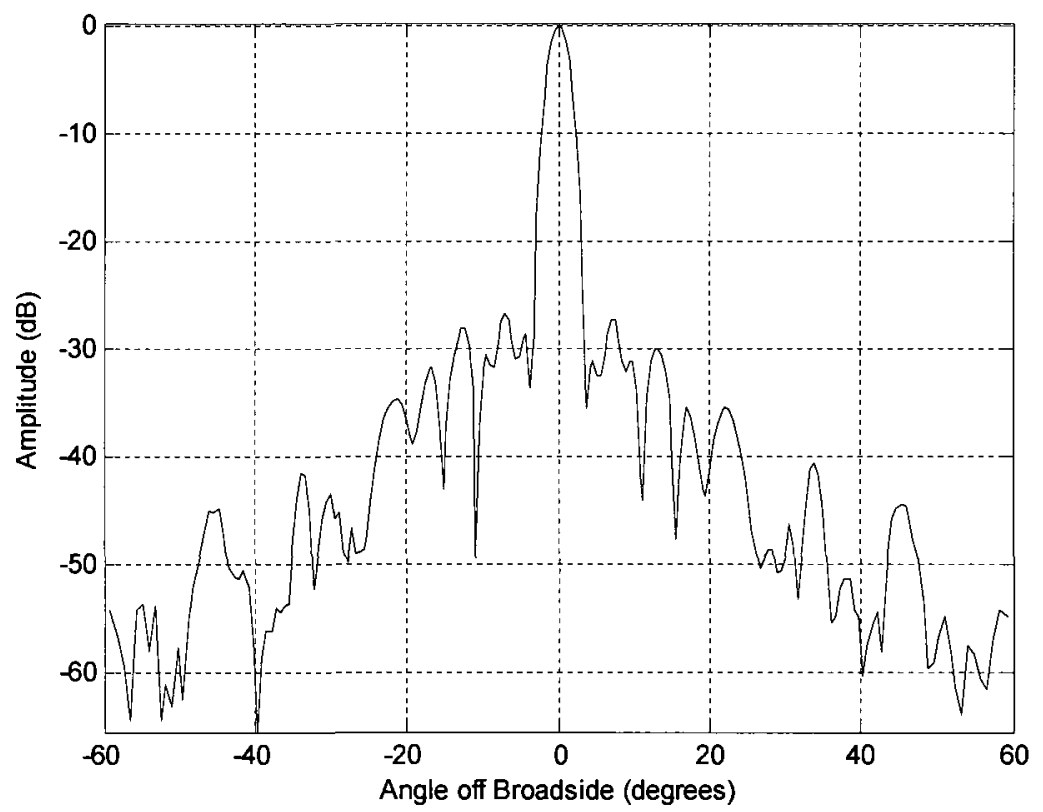

Figure 4.6-4: Normalized far-field $\left|E_{\mathrm{co}}(\theta, \phi)\right|$ of the monopulse reflector antenna for pattern cut $\phi=0^{\circ}$

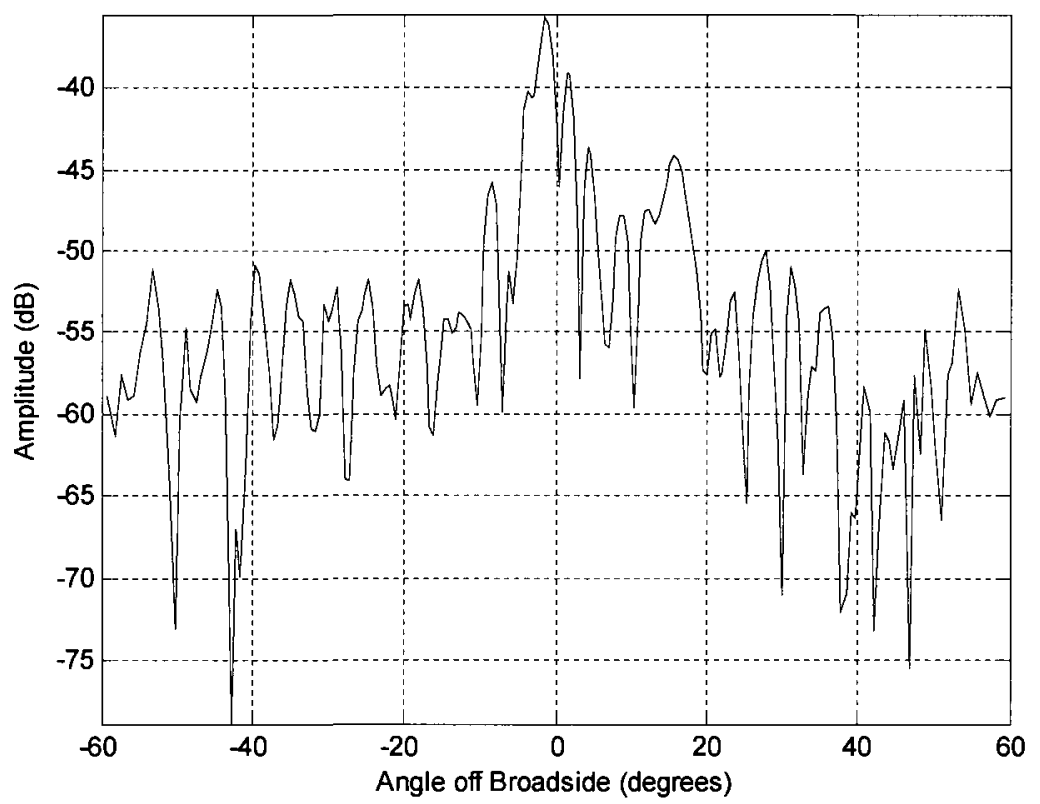

Figure 4.6-5: Far-field $\left|E_{\mathrm{cr}}(\theta, \phi)\right|$ of the monopulse reflector antenna for pattern cut $\phi=0^{\circ}$, normalized to $\left|E_{\mathrm{co}}(\theta, \phi)\right|$ 


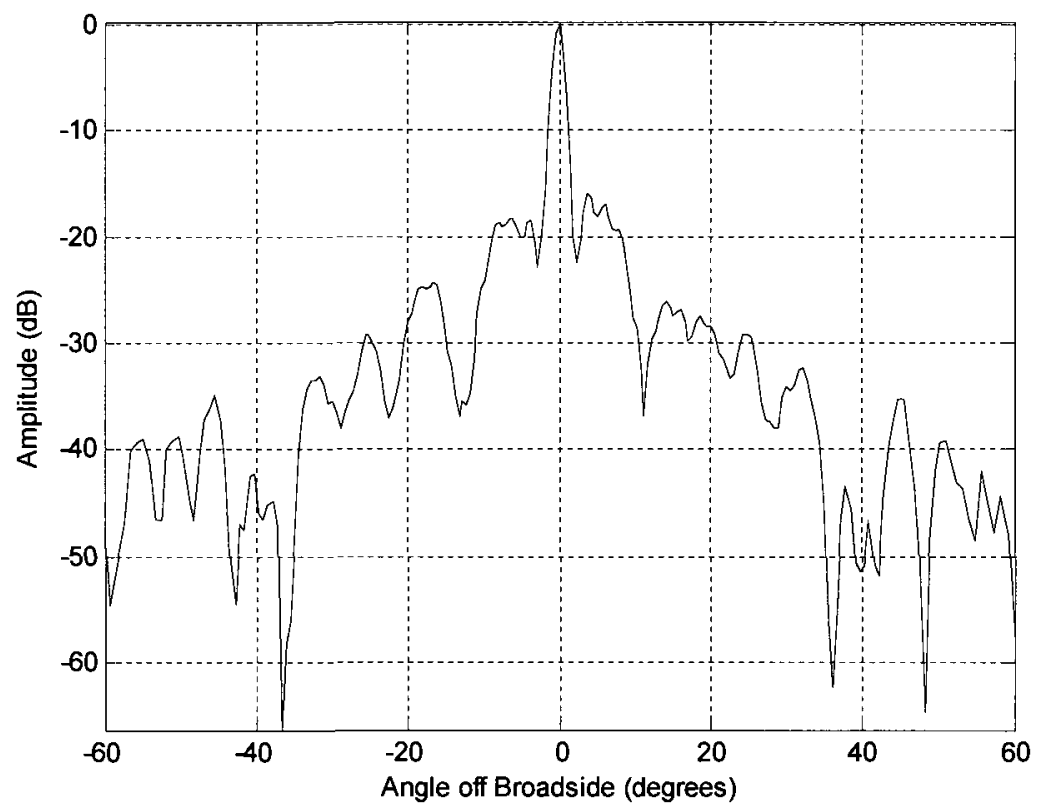

Figure 4.6-6: Normalized far-field $\left|E_{\mathrm{co}}(\theta, \phi)\right|$ of the monopulse reflector antenna for pattern cut $\phi=90^{\circ}$

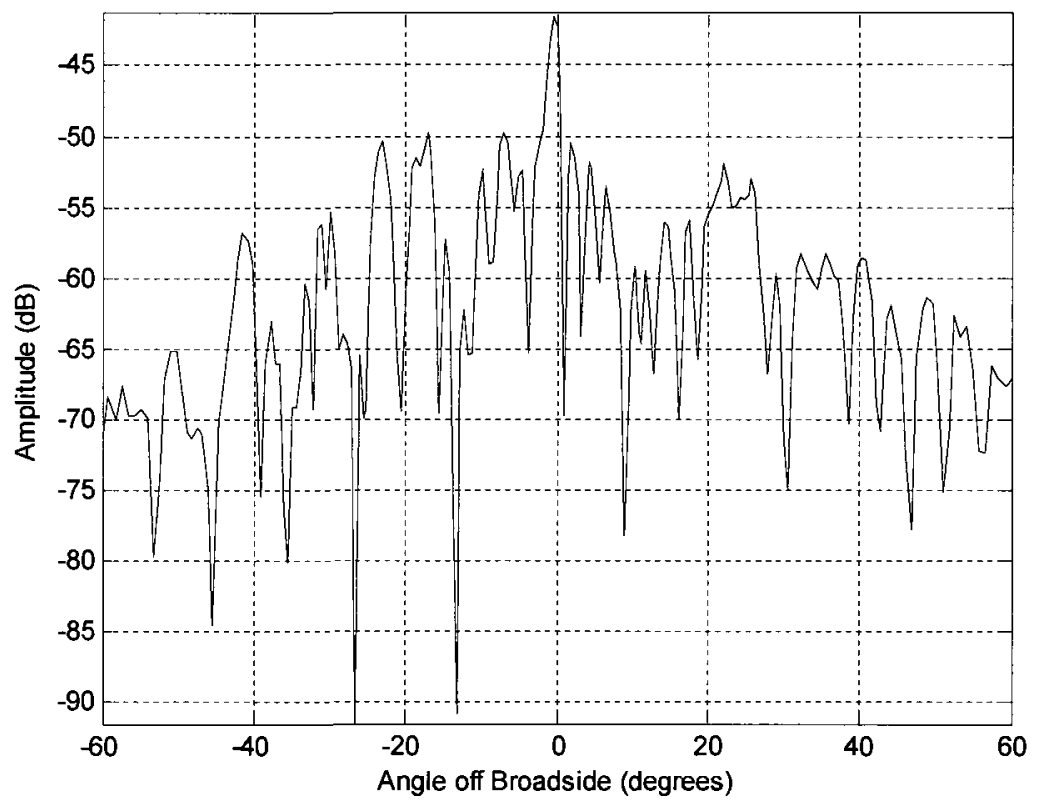

Figure 4.6-7: Far-field $\left|E_{\mathrm{cr}}(\theta, \phi)\right|$ of the monopulse reflector antenna for pattern cut $\phi=90^{\circ}$, normalized to $\left|E_{\mathrm{co}}(\theta, \phi)\right|$ 
Figure 4.6-8 shows the rule-of-thumb valid far-zone angle off broadside based on the PNF scan size (in samples per side) for the monopulse reflector antenna. The largest aperture dimension of the AUT is represented by the black vertical line.

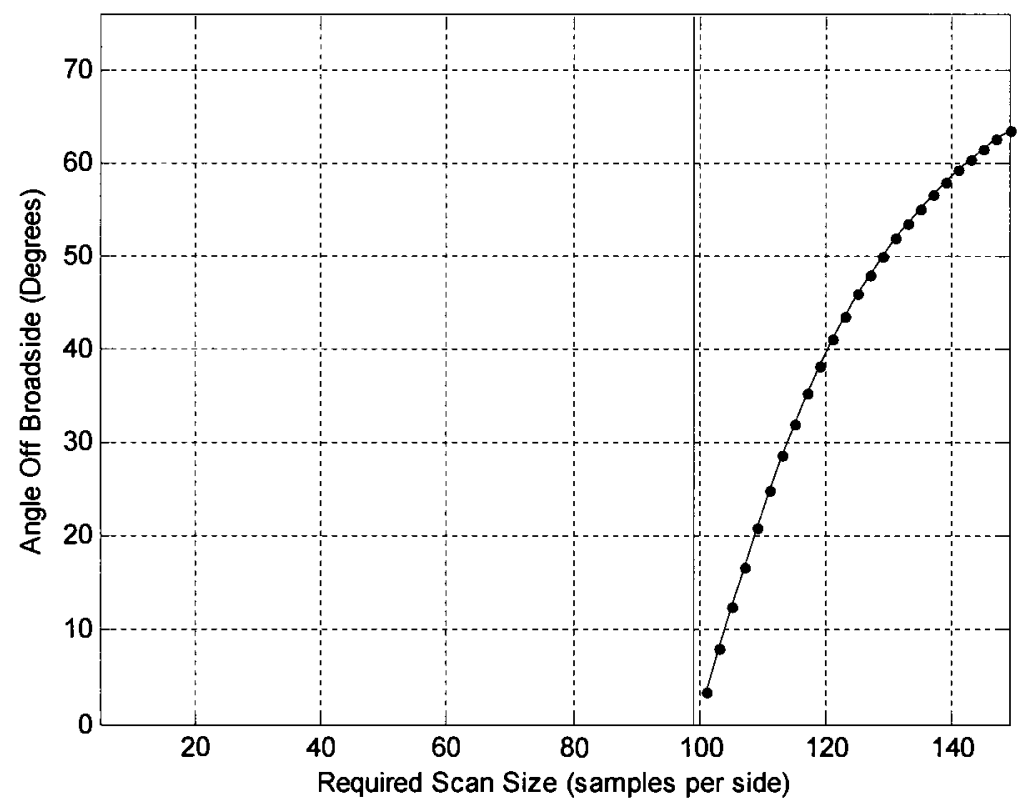

Figure 4.6-8: Rule-of-thumb valid far-zone angle off broadside for the monopulse reflector antenna. The vertical line shows the AUT aperture

\subsection{Slotted Waveguide Array}

Details of the PNF scan of the slotted waveguide array, which we will refer to as the slotted waveguide array, can be found in Table 4.7-1. The slotted waveguide array was scanned at the PNF chamber at the University of Ottawa, and was purposely scanned as far as the PNF mechanism allowed. Figure 4.7-1 and Figure 4.7-2 show the $\mathrm{x}$ and $\mathrm{y}$ components of the PNF scanned data. Figure 4.7-3 shows the calculated directivity 
versus scan size for the slotted waveguide array. Figure 4.7-4 through Figure 4.7-7 show the principal pattern cuts for the far-zone $\mathrm{E}_{\mathrm{co}}$ and $\mathrm{E}_{\mathrm{cr}}$, based on the complete PNF data set.

Table 4.7-1: PNF scan information for the slotted waveguide array

\begin{tabular}{|l|l|l|}
\hline AUT Size & Width $=0.305 \mathrm{~m}$ & Height $=0.345 \mathrm{~m}$ \\
\hline Actual PNF Scan Size & Width $=0.810 \mathrm{~m}$ & Height $=0.850 \mathrm{~m}$ \\
\hline PNF Number of Samples & $\mathrm{x}=132$ & $\mathrm{y}=138$ \\
\hline PNF Sample Spacing & $\mathrm{x}=0.00618 \mathrm{~m}$ & $\mathrm{y}=0.00621 \mathrm{~m}$ \\
\hline Probe to AUT Distance & $0.09 \mathrm{~m}$ & \\
\hline Frequency & $23.25 \mathrm{GHz}$ & Probe $2=$ Ey \\
\hline PNF Probe Polarization & Probe $1=\mathrm{Ex}$ & $70^{\circ}, \mathrm{y}$ \\
\hline Rule-of-thumb Far-Field Angle & $70^{\circ}, \mathrm{x}$ & \\
\hline Calculated Directivity & $37.48 \mathrm{dBi}$ & \\
\hline Principle Near-Field Component & $\mathrm{E}_{\mathrm{x}}$ \\
\hline
\end{tabular}




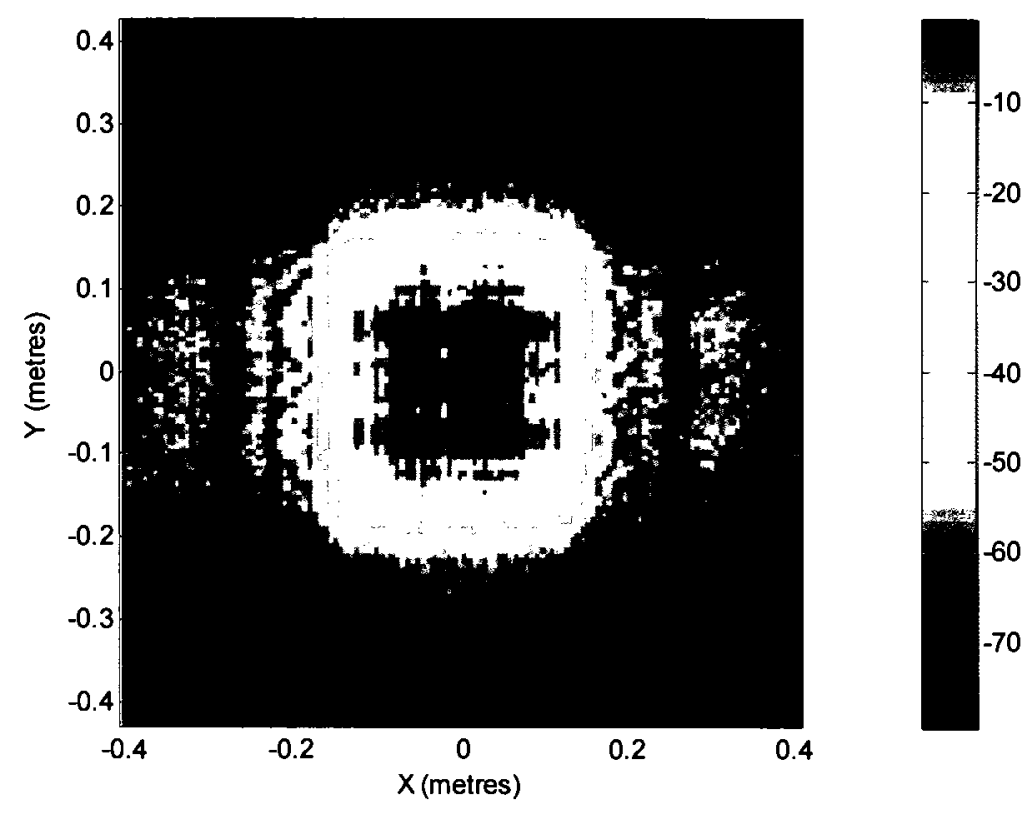

Figure 4.7-1: Normalized near-field $\left|E_{\mathrm{x}}(x, y)\right|$ sampled over the scan plane for the slotted waveguide array, in $\mathrm{dB}$

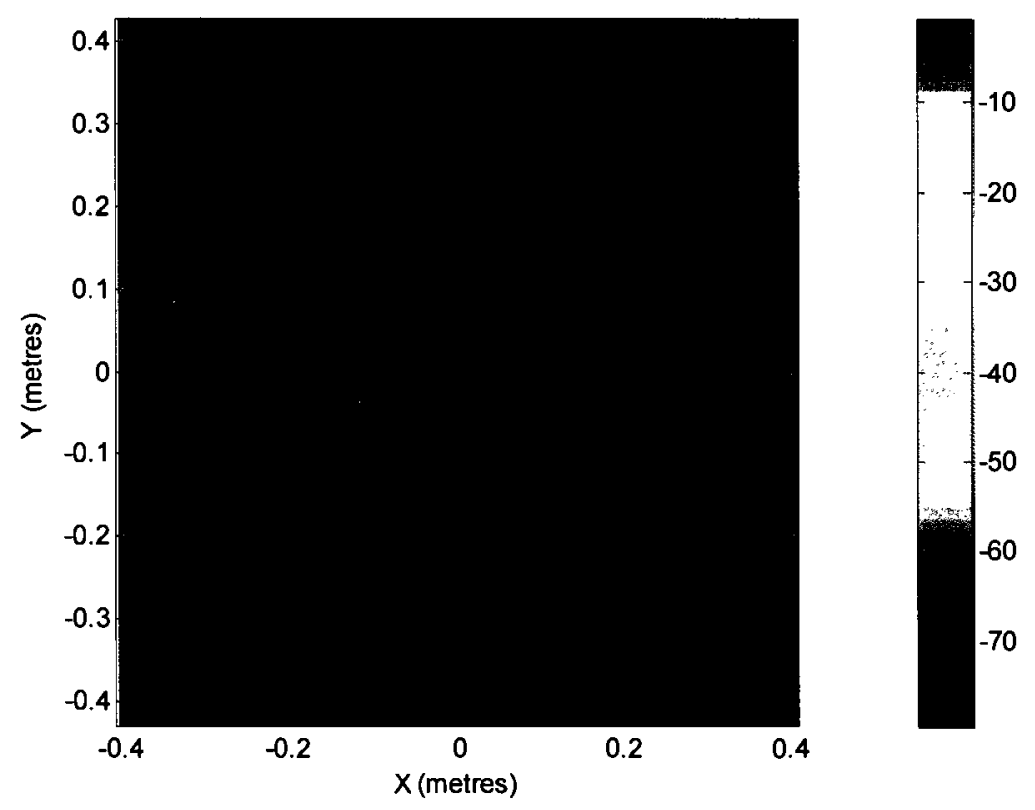

Figure 4.7-2: Near-field $\left|E_{y}(x, y)\right|$ sampled over the scan plane for the slotted waveguide array, normalized to $E_{\mathrm{x}}$, in $\mathrm{dB}$ 


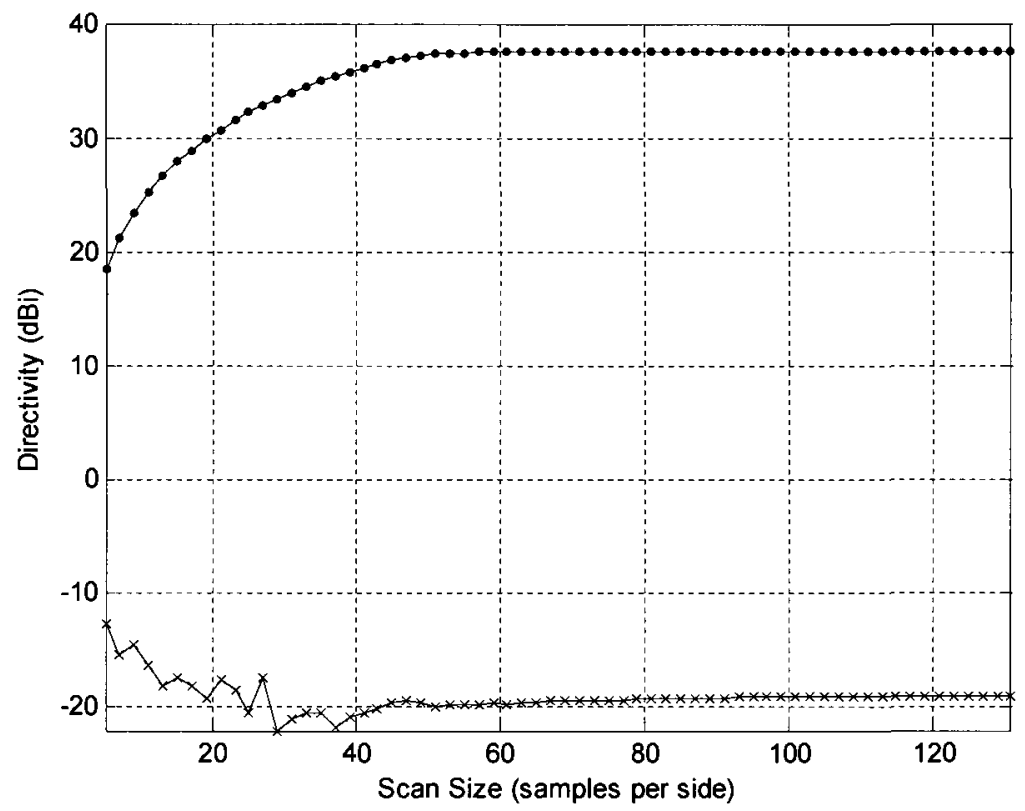

Figure 4.7-3: Co (o) and cross (x) polarized directivity of slotted waveguide array based on near-field scan size

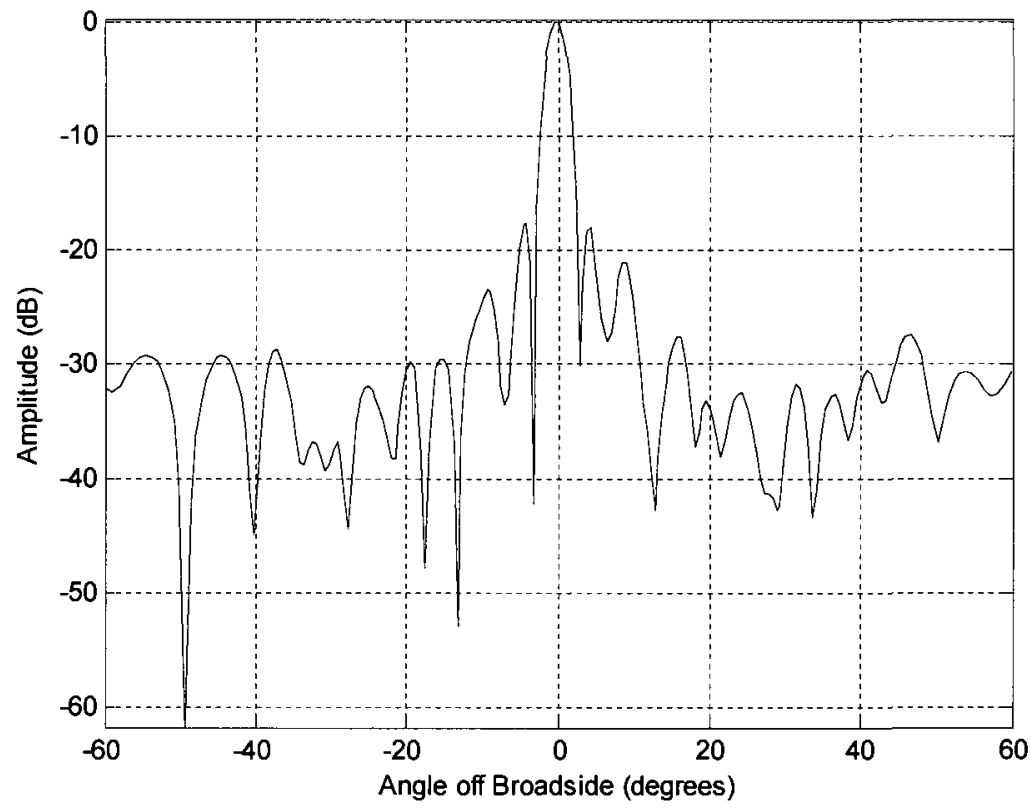

Figure 4.7-4: Normalized far-field $\left|E_{\mathrm{co}}(\theta, \phi)\right|$ of the slotted waveguide array for pattern cut $\phi=0^{\circ}$ 


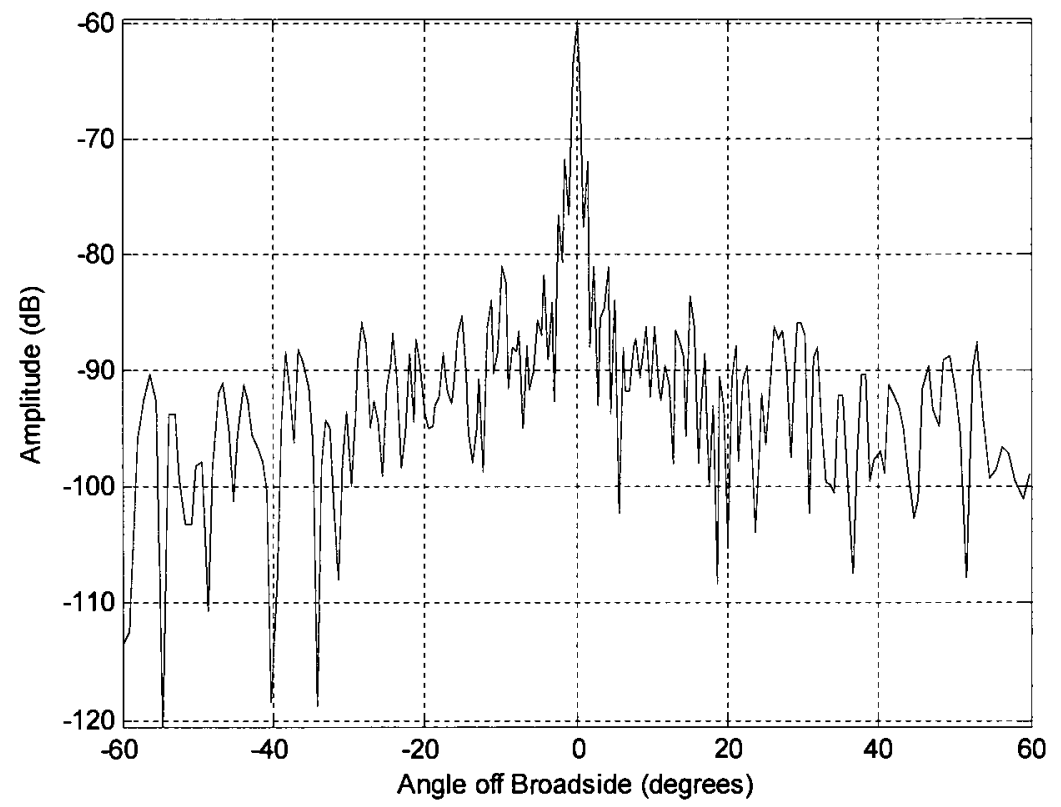

Figure 4.7-5: Far-field $\left|E_{\mathrm{cr}}(\theta, \phi)\right|$ of the slotted waveguide array for pattern cut $\phi=$ $0^{\circ}$, normalized to $\left|E_{\mathrm{co}}(\theta, \phi)\right|$

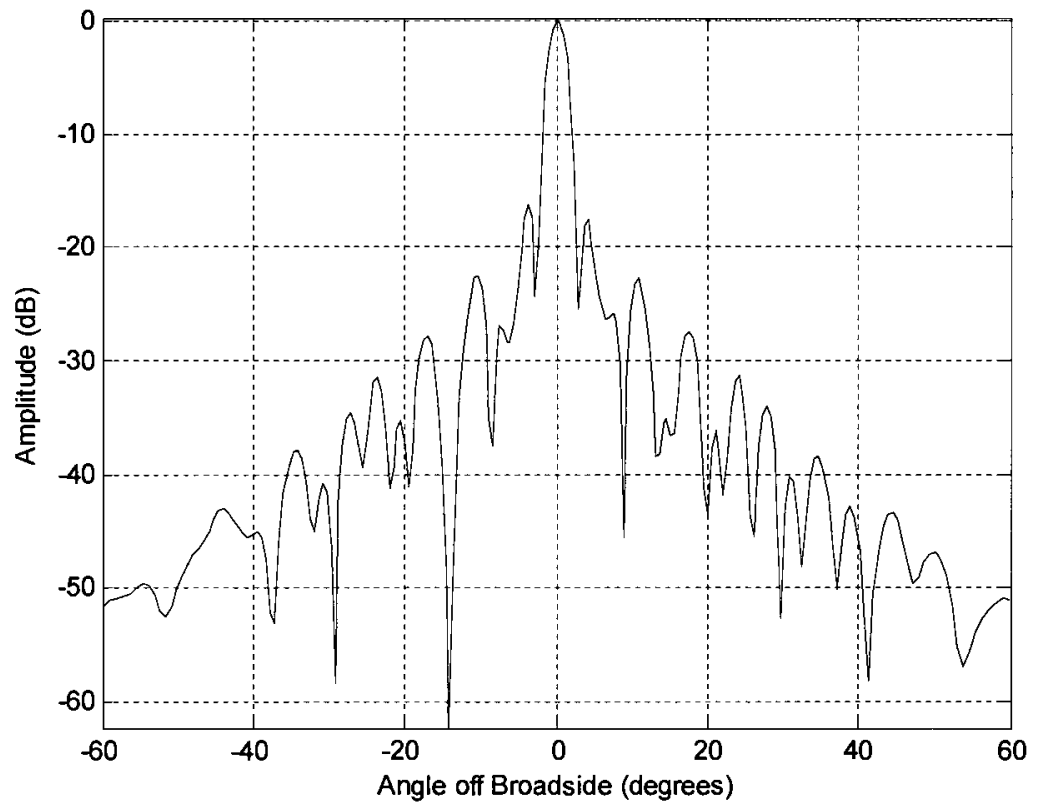

Figure 4.7-6: Normalized far-field $\left|E_{\mathrm{co}}(\theta, \phi)\right|$ of the slotted waveguide array for pattern cut $\phi=90^{\circ}$ 


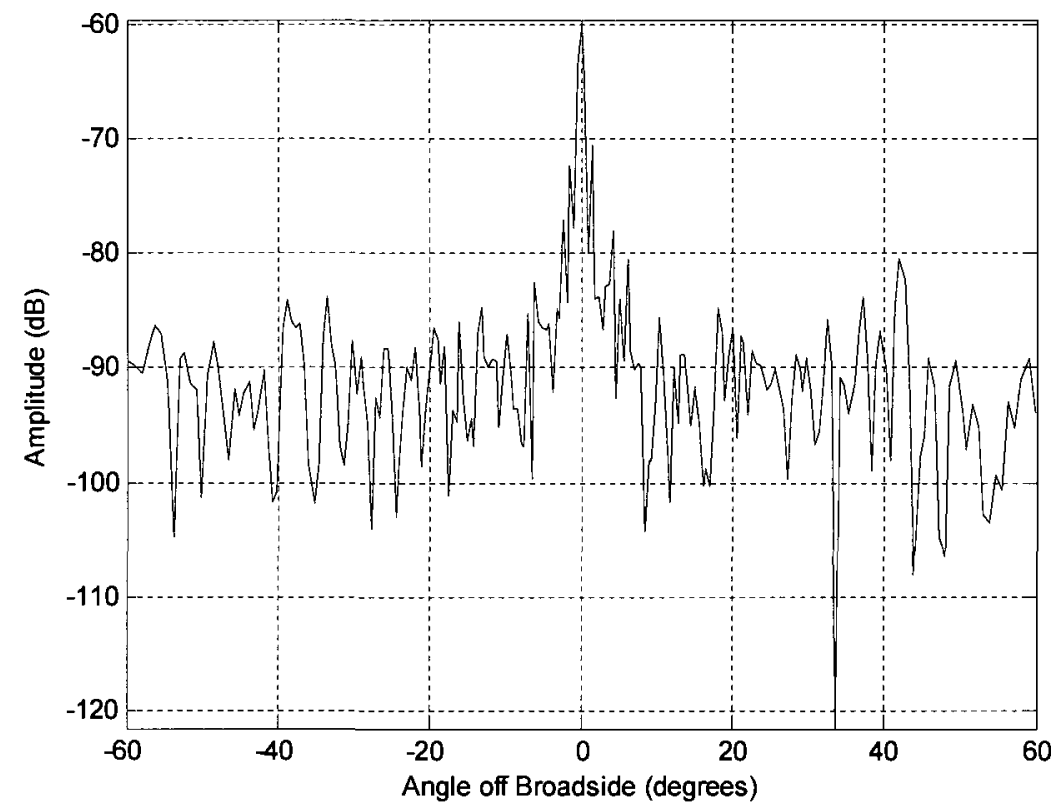

Figure 4.7-7: Far-field $\left|E_{\mathrm{cr}}(\theta, \phi)\right|$ of the slotted waveguide array for pattern cut $\phi=$ $\mathbf{9 0}^{\circ}$, normalized to $\left|E_{\mathrm{co}}(\theta, \phi)\right|$

Figure 4.7-8 shows the rule-of-thumb valid far-zone angle off broadside based on the PNF scan size (in samples per side) for the slotted waveguide array. The largest aperture dimension of the AUT is represented by the black vertical line. 


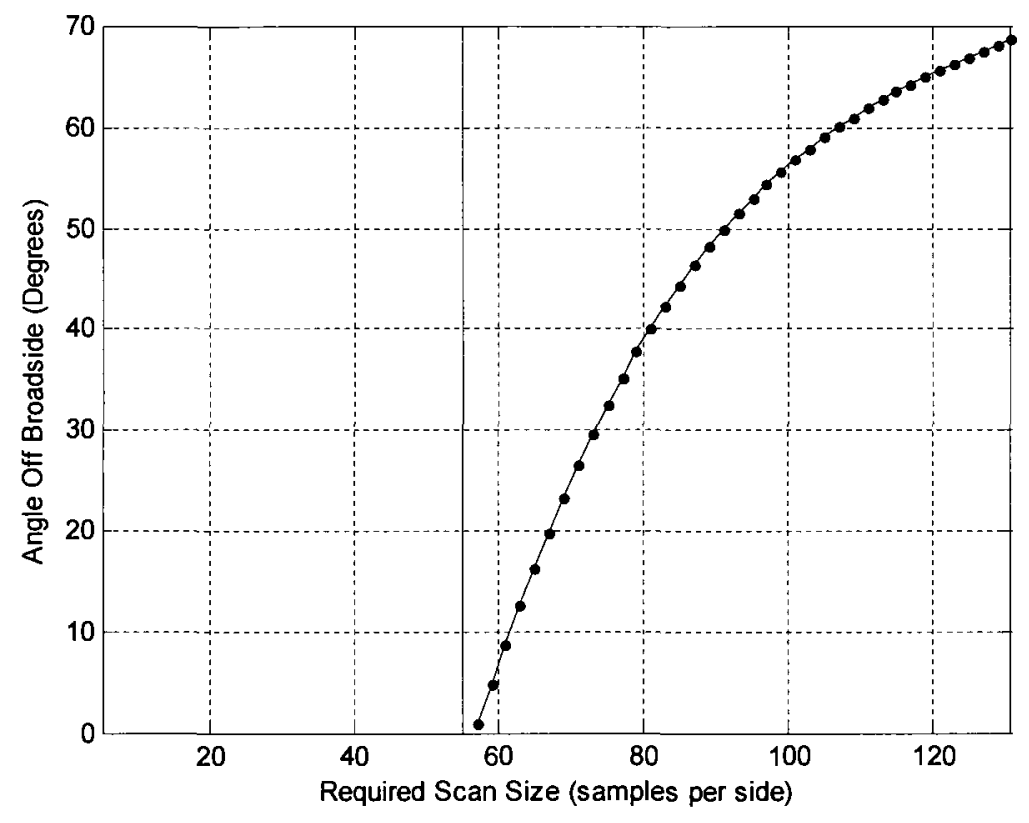

Figure 4.7-8: Rule-of-thumb valid far-zone angle off broadside for the slotted waveguide array. The vertical line shows the AUT aperture.

\subsection{Concluding Remarks}

In this chapter we have presented the antennas that will be used to evaluate the decision functions in experiments described in Chapter 5. The PNF data sets for the antennas presented in this chapter, with the exception of the slotted waveguide array which was scanned in the PNF facility at the University of Ottawa, were provided courtesy of Nearfield Systems Inc. (NSI), California, USA. 


\section{CHAPTER 5 - COMPARATIVE STUDY OF DECISION FUNCTIONS FOR ADAPTIVE PLANAR NEAR-FIELD TECHNIQUES}

\subsection{Introduction}

In this chapter we explore the decision functions devised in Section 3.4 by applying them to the series of PNF data sets of the antennas reviewed in Chapter 4. Some initial comments about this comparative study are given in Section 5.2. Section 5.3 outlines the experiments that were performed on the near-field data sets. The results and discussions of these experiments are presented in Section 5.4 through 5.7. Concluding remarks for this chapter are provided in Section 5.8, and the references for Chapter 5 are given in Section 5.9.

\subsection{Preliminary Comments}

As previously noted, the selected antennas cover a range of types commonly tested using PNF techniques. The goal of these experiments is to examine the behaviour of the decision functions. In so doing we will show the potential for using these to select a stopping requirement for the adaptive acquisition method devised in Chapter 3 . This will give us insight into how these decision functions could be used in practice during actual PNF testing. The decision of when to stop the PNF scan is not trivial and the characteristics of the AUT, as well knowledge of PNF measurements, need to be taken into consideration to make this determination. 
There are nine decision functions in total and the decision of when to terminate the PNF scan will not necessarily depend on the performance of all nine. Oftentimes the purpose of conducting a PNF scan is to verify a particular pattern characteristic of the AUT, such as the level of a particular sidelobe or the maximum directivity. An operator conducting a PNF test would have sufficient knowledge to be able to realistically set thresholds and stopping conditions for the adaptive acquisition method to achieve the test objectives.

\subsection{Experiment Set-up}

The experiments whose outcomes are presented in the remaining sections of this chapter were all carried out using the following approach: Complete (and large) measured PNF data sets for the individual AUTs were first obtained. Such data is made available to the code $A P N F$ developed in the MATLAB environment. This code simulates the scanning sequence described in Section 3.2 by simply reading the data points from the large data file in the order in which it would be sequentially acquired in practice if the adaptive method were being used. At the end of each new scan iteration the far-zone fields and decision functions are calculated. The decision functions are plotted as the scan iterations proceed until the limits of the large PNF data set are reached.

The PNF scan area as used by $A P N F$ is square ${ }^{22}$, and its size is indicated in "samples per side". Thus if the number of samples per side is $M$, and the spacing between

\footnotetext{
${ }^{22}$ The $A P N F$ reads the input PNF data set (which are not necessarily square) and implements a square adaptive scanning pattern. The $A P N F$ could easily be modified to implement a rectangular scan plane, which could be based on the shape of the aperture of the AUT (such as the monopulse reflector antenna).
} 
samples along any axis is $\lambda P$, then the scan area is $[(M-1) \lambda P]^{2}$. The scan area need not be square in order for the adaptive PNF method to be applied.

Not only are the various decision functions examined, but the behaviour of a given decision function is observed over constrained "regions of interest". This is achieved by altering the weighting functions $w_{\mathrm{co}}(\theta, \phi)$ and $w_{\mathrm{cr}}(\theta, \phi)$ of Section 3.4, in order to determine how the required PNF scan size changes as the region of interest is constrained. If $w_{\mathrm{co}}(\theta, \phi)=30^{\circ 23}$, for instance, then only those far-zone pattern points in directions less than or equal to $30^{\circ}$ off broadside are included in the computation of the decision function. We will call such an example a $\zeta=30^{\circ}$ case, and so on. In conventional PNF testing the rule-of-thumb expression (2.4-1) would give the required scan size for a specified value of $\zeta$; in this thesis we are of course proposing the use of decision functions in a feedback loop to determine what the PNF scan size needs to be for a desired $\zeta$

The decision functions are presented separately in terms of their co-polarized and cross-polarized components. This relates more directly to how the information is actually used in practice. The co-polarized components of the decision functions are the default case and are those decision functions that will be of most interest in practice. The crosspolarized components will only be presented for certain cases where some specific aspect needs to be highlighted. This cross-polarized analysis is presented for the offset reflector antenna in Section 5.7. The evaluation of the decision functions will use the considerations in the discussion that follows.

\footnotetext{
${ }^{23}$ Here the $30^{\circ}$ refers to the $\theta$ angle. We do not place a constraint on the $\phi$ angle.
} 
We will show the effects of varying $\zeta$ for the chosen decision functions. In a traditional PNF scan the desired far-zone region of validity angle, $\zeta$, will determine how large the PNF scan plane must be, based on the rule-of-thumb calculation.

Two possibilities for deciding when to terminate the PNF scans will be discussed, namely:

- The value of the decision function falls below some threshold value. The choice of threshold will ultimately depend on the AUT and the goals of the PNF scan.

- The decision function has "settled down" to a value that changes very little as the PNF scan size increases.

The following is a list of parameters set for all the experiments that follow:

- $\mathrm{P}_{\mathrm{DFE}, \mathrm{co}}$ and $\mathrm{P}_{\mathrm{DFE}, \mathrm{cr}}$ were set to $-100 \mathrm{~dB}$

- $\mathrm{P}_{\mathrm{DFD}, \mathrm{co}}$, and $\mathrm{P}_{\mathrm{DFD}, \mathrm{cr}}$ were set to $-50 \mathrm{~dB}$

- $\mathrm{P}_{\mathrm{DFN}, \mathrm{x}}$, and $\mathrm{P}_{\mathrm{DFN}, y}$ were set to $-50 \mathrm{~dB}$

- $\zeta$ was set to $60^{\circ}, 30^{\circ}, 15^{\circ}$, and $5^{\circ}$ off broadside

In the decision function plots presented in the sections that follow, the black vertical line represents the largest dimension of the AUT physical aperture, and the ruleof-thumb stopping size for each of the required region of validity angle values, $\zeta$, (included as an inset in each figure ${ }^{24}$ ) is shown by the asterisk (*) data marker.

\footnotetext{
${ }^{24}$ In the figures that follow, if the plots for the different $\zeta$ overlap each other only the colour of the smallest $\zeta$ is shown (this plot is on top of the others). In other words, if the plots for all $\zeta$ are the same only the colour for $\zeta=5^{\circ}$ is shown. If $\zeta=60^{\circ}$ and $30^{\circ}$ are the same then only the colour of the $\zeta=30^{\circ}$ is displayed.
} 


\subsection{Analysis of Decision Functions DFEn}

\subsubsection{Analysis of Decision Function DFE1}

Figure 5.4-1 through Figure 5.4-5 show plots of DFE1 versus scan size for each of the antennas under examination. DFE1 is an averaged error measure, and so provides curves that smoothly taper off as the scan size increases. Reducing the size of the region of interest reduces the overall value of the co-polarized component of DFE1, but the shapes of the plots remain similar. The problem is that it is difficult to decide on a threshold value to be used with DFE1 because it is not directly related to a quantity in terms of which antenna performance specifications are written or through which the accuracy of these quantities can be expressed. Only for the monopulse reflector antenna and slotted waveguide array cases of Figure 5.4-4 and Figure 5.4-5 does DFE1 flatten out as the scan size increases. The scan size at which this flattening out begins might be used as a scan termination point for this specific antenna. However, the lack of such behaviour for the other antennas means that DFE1 is not on its own a useful decision function in general $^{25}$. It is interesting that for the $\xi=60^{\circ}$ case of the slotted waveguide array the flattening out begins at a scan size that is smaller than that required by the rule-of-thumb. The rule-of-thumb size required for the $\xi$ angles are shown in each figure by the ${ }^{*}$ data marker ${ }^{26}$.

\footnotetext{
${ }^{25}$ But see further comments in Section 5.7 on the possible use of DFE1.

${ }^{26}$ If the * data marker does not appear on a particular $\xi$ plot, it means that that $\xi$ angle is not possible from the acquired PNF data set based on the rule-of-thumb calculation. The PNF data set would need to be scanned further to include these $\xi$ angles.
} 


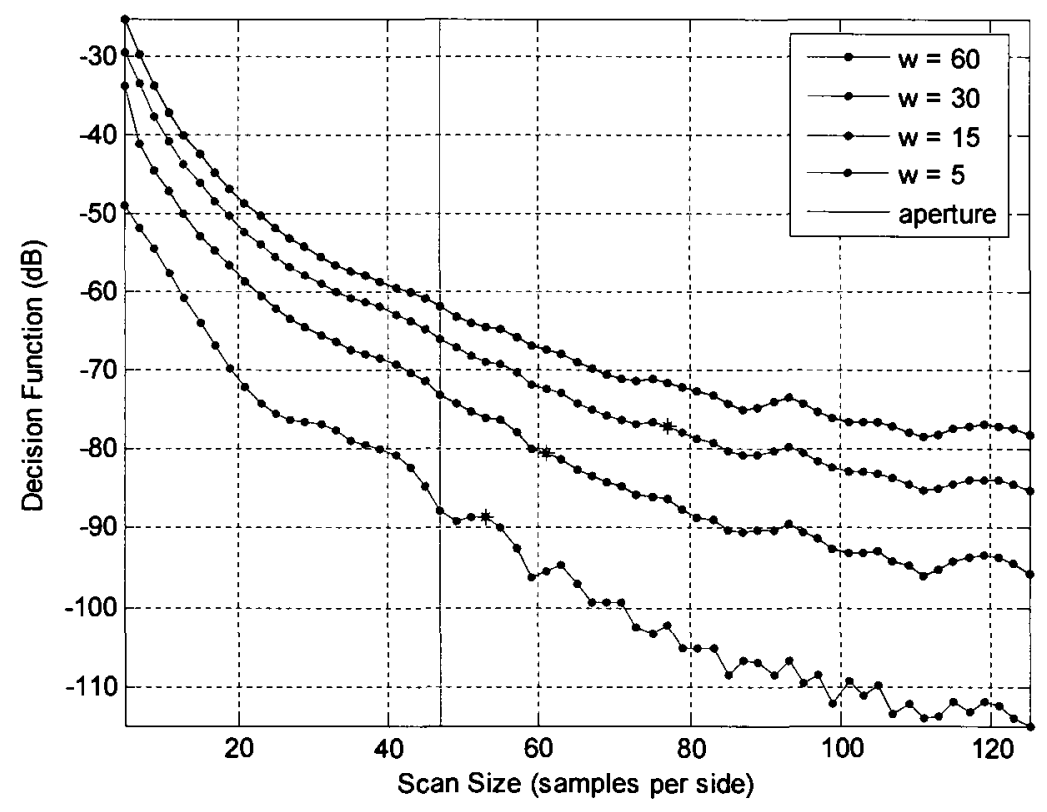

Figure 5.4-1: Co-polarized component of DFE1 for the offset reflector antenna

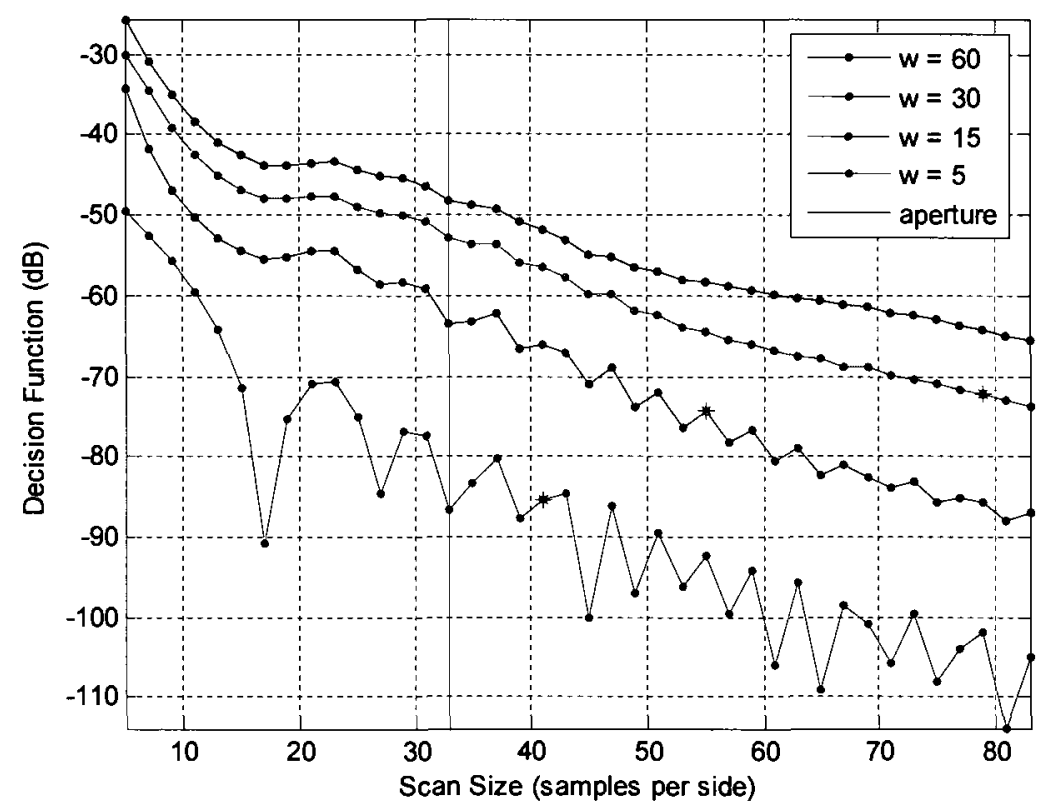

Figure 5.4-2: Co-polarized component of DFE1 for the horn antenna 


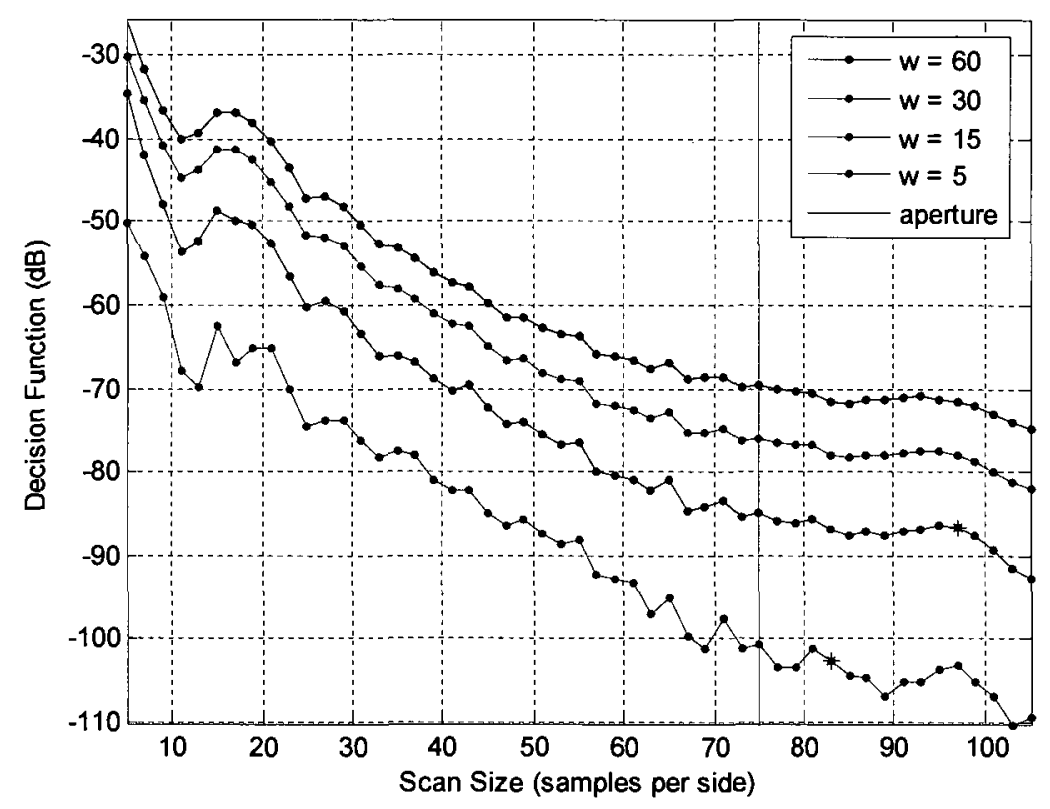

Figure 5.4-3: Co-polarized component of DFE1 for the planar array

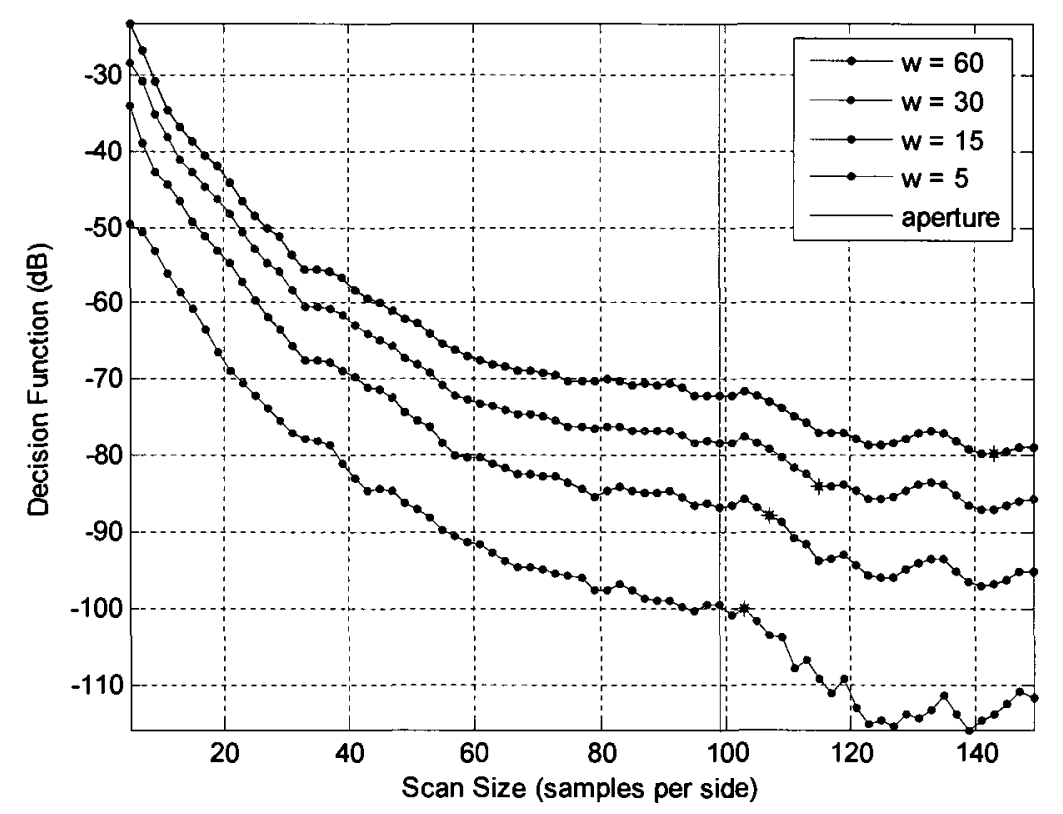

Figure 5.4-4: Co-polarized component of DFE1 for the monopulse reflector antenna 


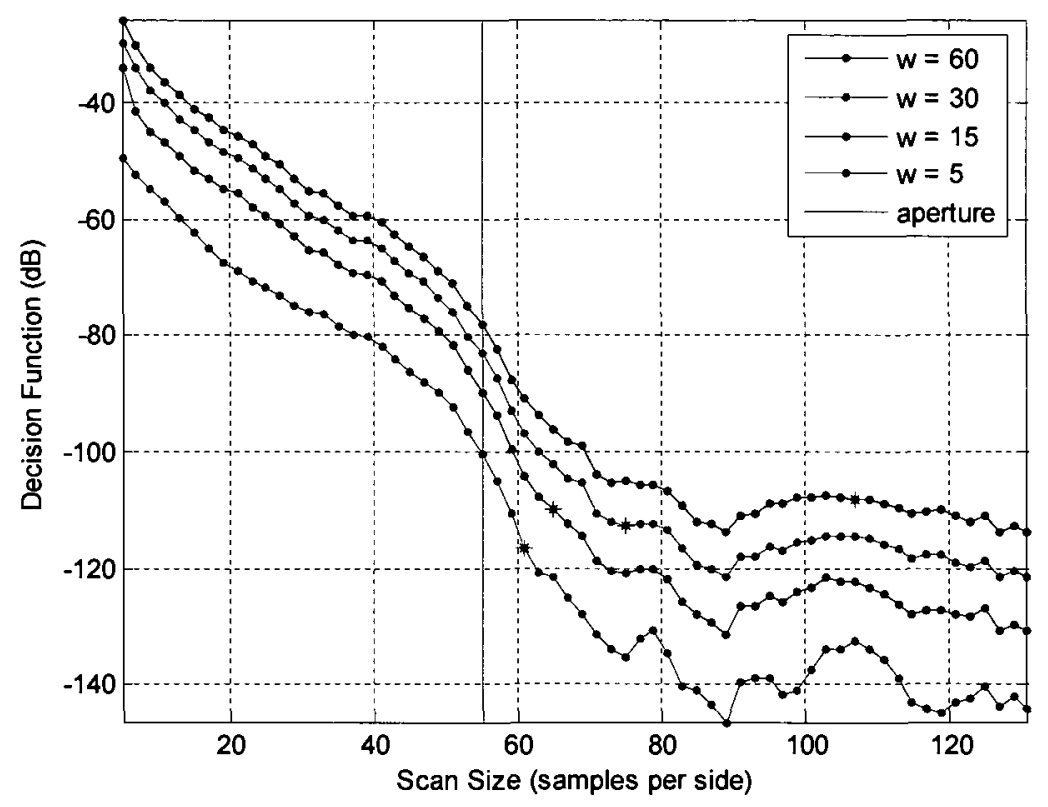

Figure 5.4-5: Co-polarized component of DFE1 for the slotted waveguide array

\subsubsection{Analysis of Decision Function DFE2}

Decision function DFE2 is more easily used to set a threshold value than DFE1, as will be seen by inspecting Figure 5.4-6 through Figure 5.4-10 that show plots of DFE2 versus scan size for each of the antennas under examination. Only the co-polarized cases are considered, for reasons given in Section 5.3. For instance, Figure 5.4-6 shows that the DFE2 of the offset reflector antenna drops below $-50 \mathrm{~dB}$ for a scan size of about 83 samples per side. Since DFE2 is the largest value of the difference measure over the stated region of interest, it means (from Table 3.4-1) that the uncertainty is better than $\pm 0.5 \mathrm{~dB}$ down to the $-25 \mathrm{~dB}$ pattern level and better than $\pm 0.1 \mathrm{~dB}$ down to the $-10 \mathrm{~dB}$ level. Similar comments can be made for the other antennas under examination. Quantity DFE2 is thus something that can be used by experienced antenna engineers as a practical 
decision function. We observe from Figure 5.4-7 that for the horn antenna the DFE2 curve does not decrease monotonically as the scan size increases. However, it's envelope does decrease and so the same considerations can be applied to this case as for the other antennas being examined.

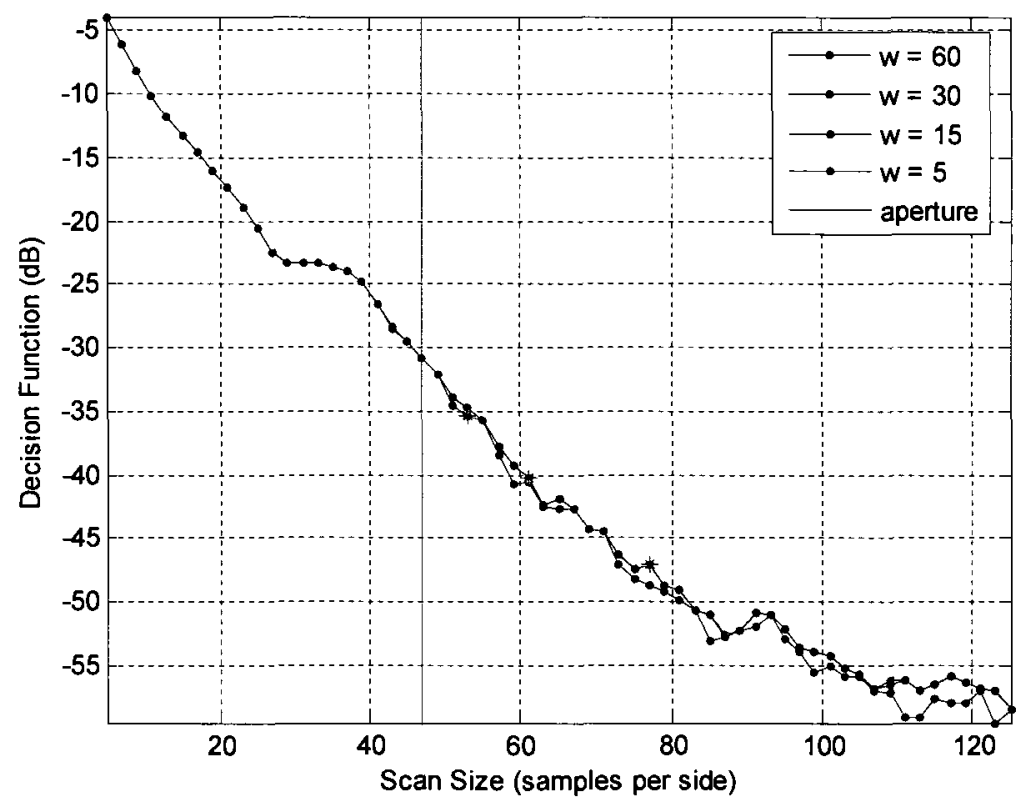

Figure 5.4-6: Co-polarized component of DFE2 for the offset reflector antenna 


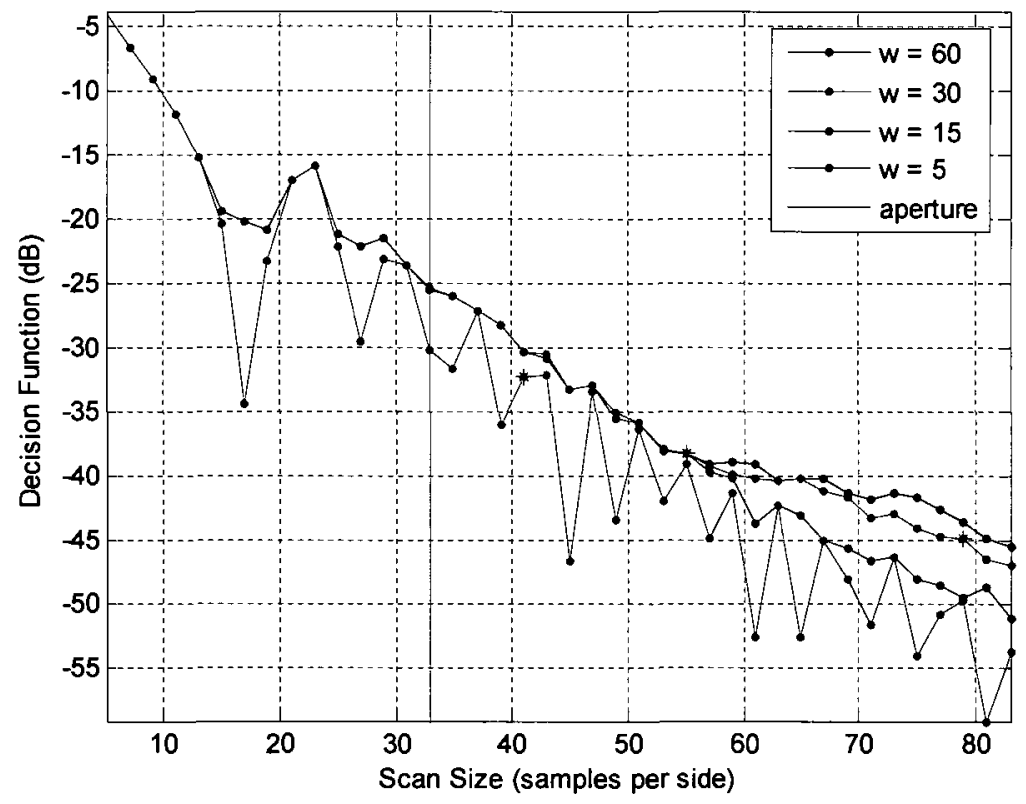

Figure 5.4-7: Co-polarized component of DFE2 for the horn antenna

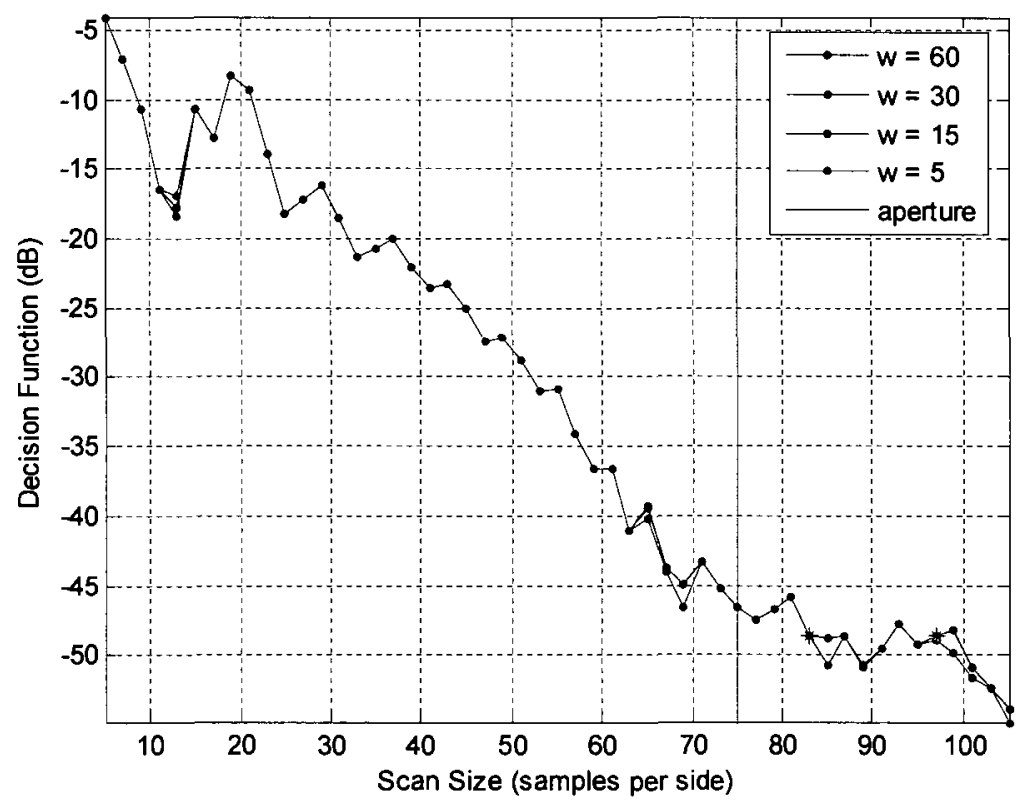

Figure 5.4-8: Co-polarized component of DFE2 for the planar array 


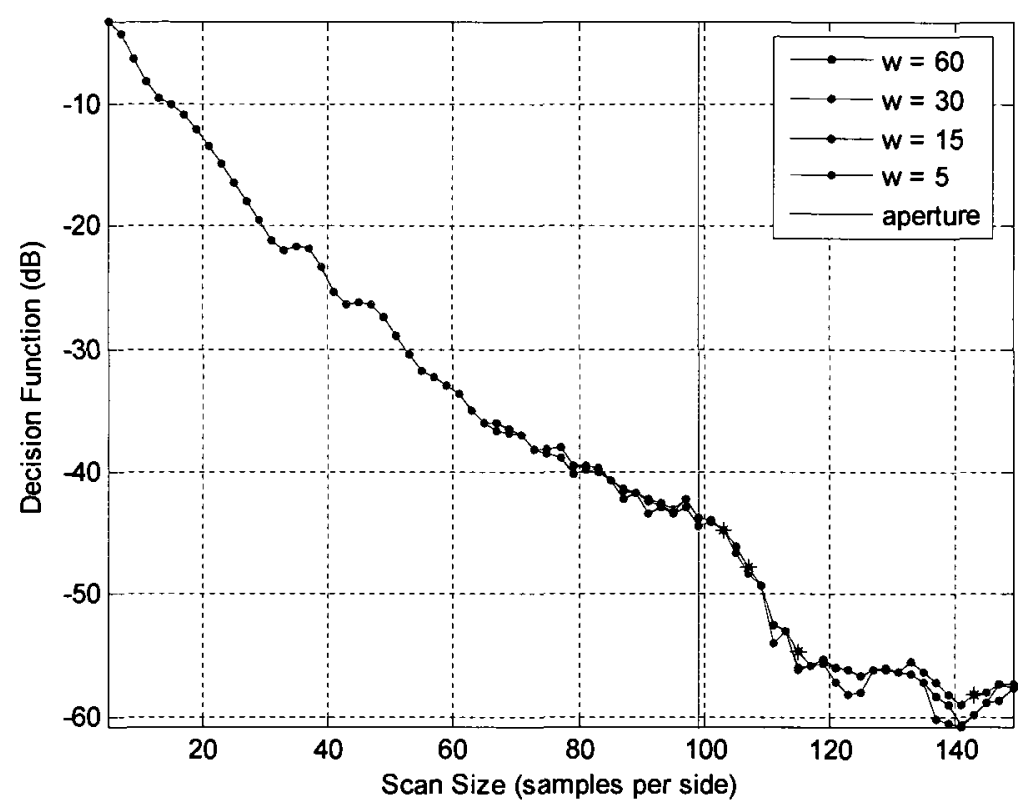

Figure 5.4-9: Co-polarized component of DFE2 for the monopulse reflector antenna

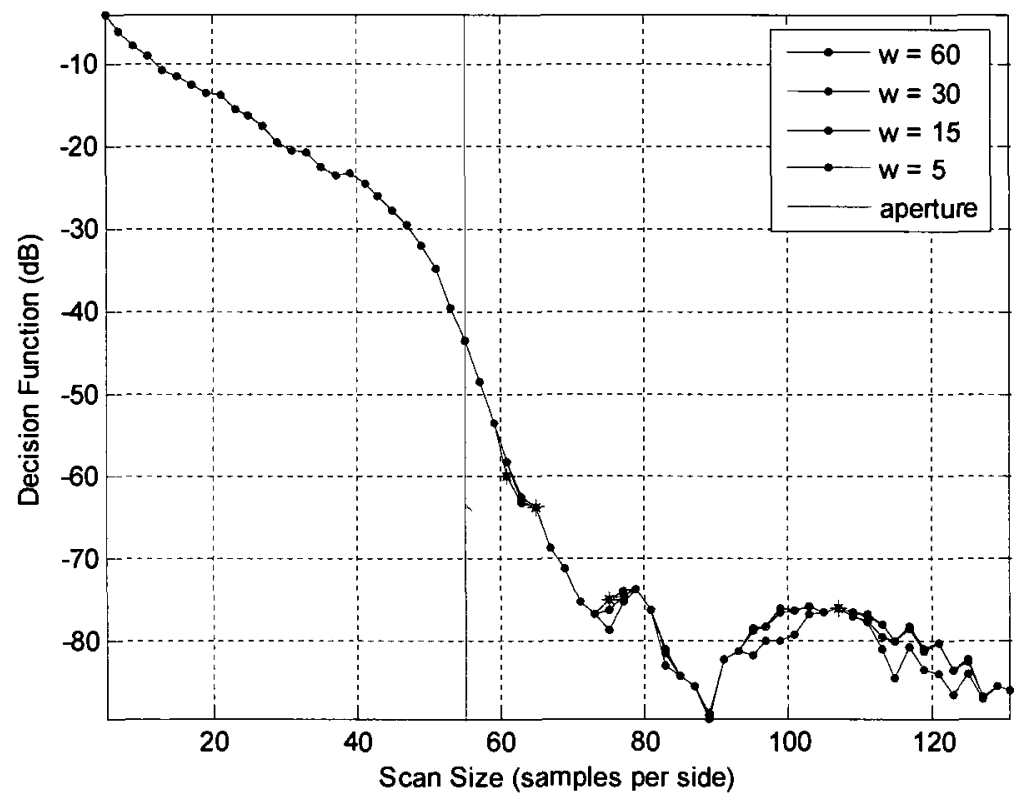

Figure 5.4-10: Co-polarized component of DFE2 for the slotted waveguide array 


\subsubsection{Analysis of Decision Function DFE3}

Decision function DFE3 shows the error at the location of the maximum far-zone co-polarized radiated pattern. Figure 5.4-11 through Figure 5.4-15 show the plot of DFE3 versus scan size for each antenna. As with DFE1 and DFE2, only the co-polarized cases are considered. In the figures below, changing $\xi$ has no $\operatorname{effect}^{27}$ on DFE3. This indicates that the location of the maximum error is the same for all $\xi$, and thus DFE3 returns the same value for that antenna. The plots for DFE3 are also noisier than the other DFE, although the envelope of the plots have the same shape as DFE2. For some PNF test scenarios the error at the far-zone pattern maximum may be an important characteristic to monitor. However, the PNF scan would not be terminated on this value alone as it says nothing about the errors at other far-zone angles.

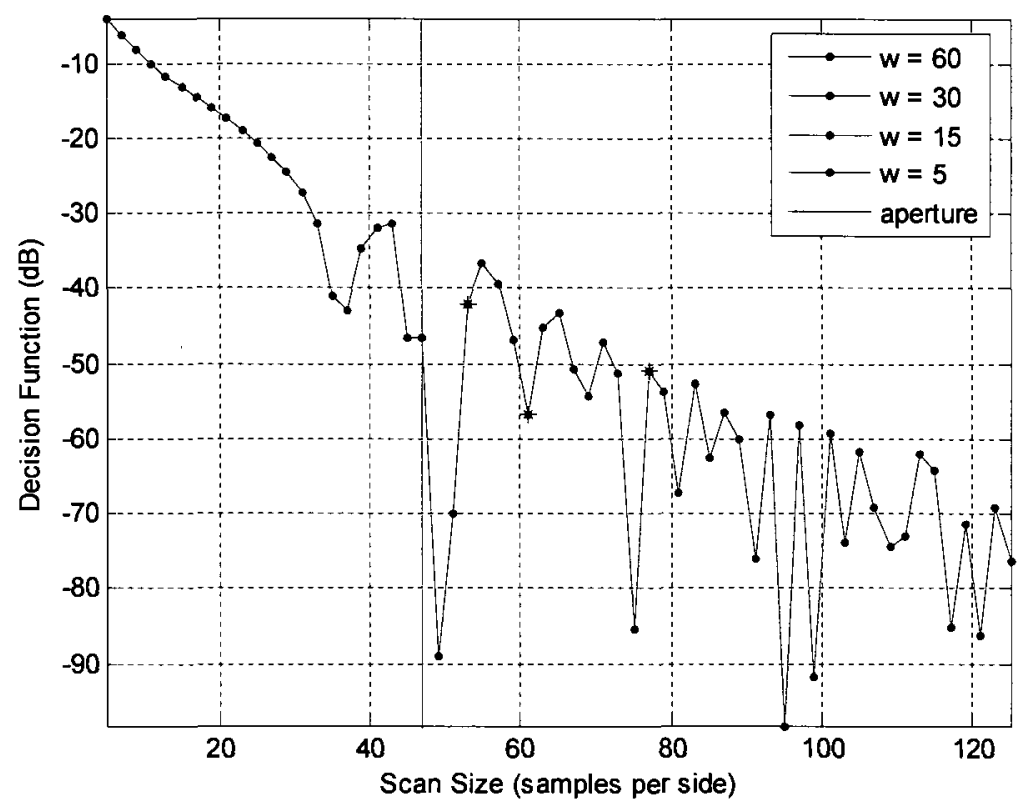

Figure 5.4-11: Co-polarized component of DFE3 for the reflector antenna

\footnotetext{
${ }^{27}$ There is s small difference for the planar array in Figure 5.4-13 because the far-zone pattern maximum based on PNF scan sizes between 11 and 21 samples per side is outside the $\xi=5$ region.
} 


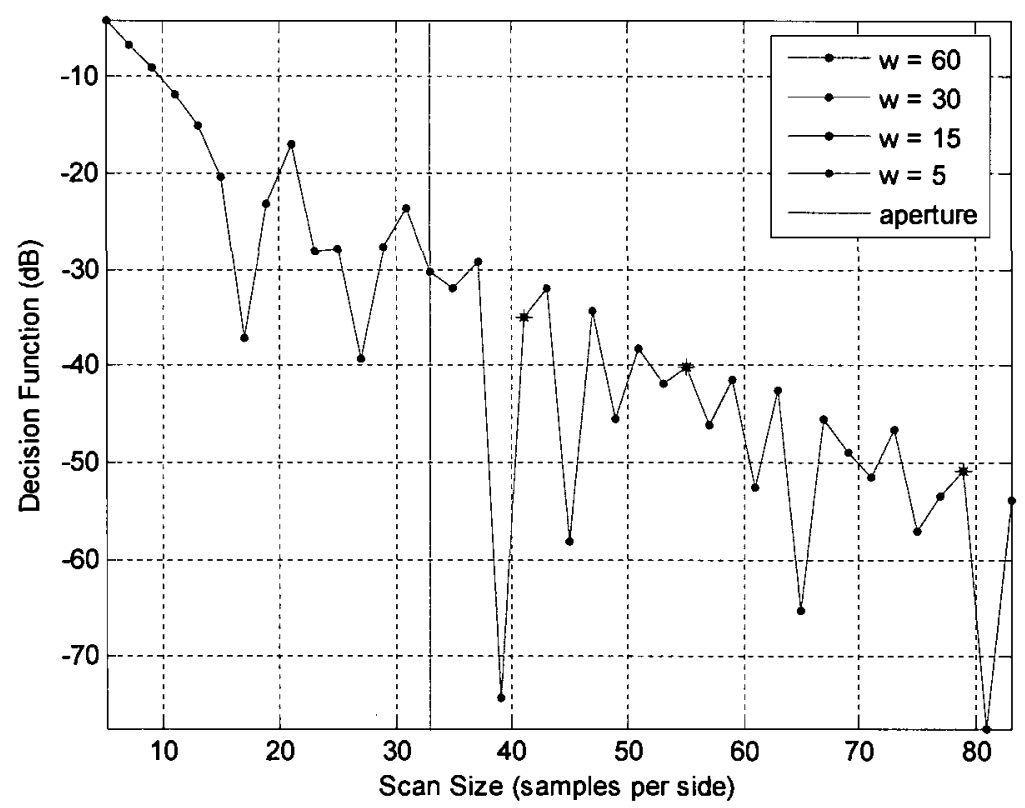

Figure 5.4-12: Co-polarized component of DFE3 for the horn antenna

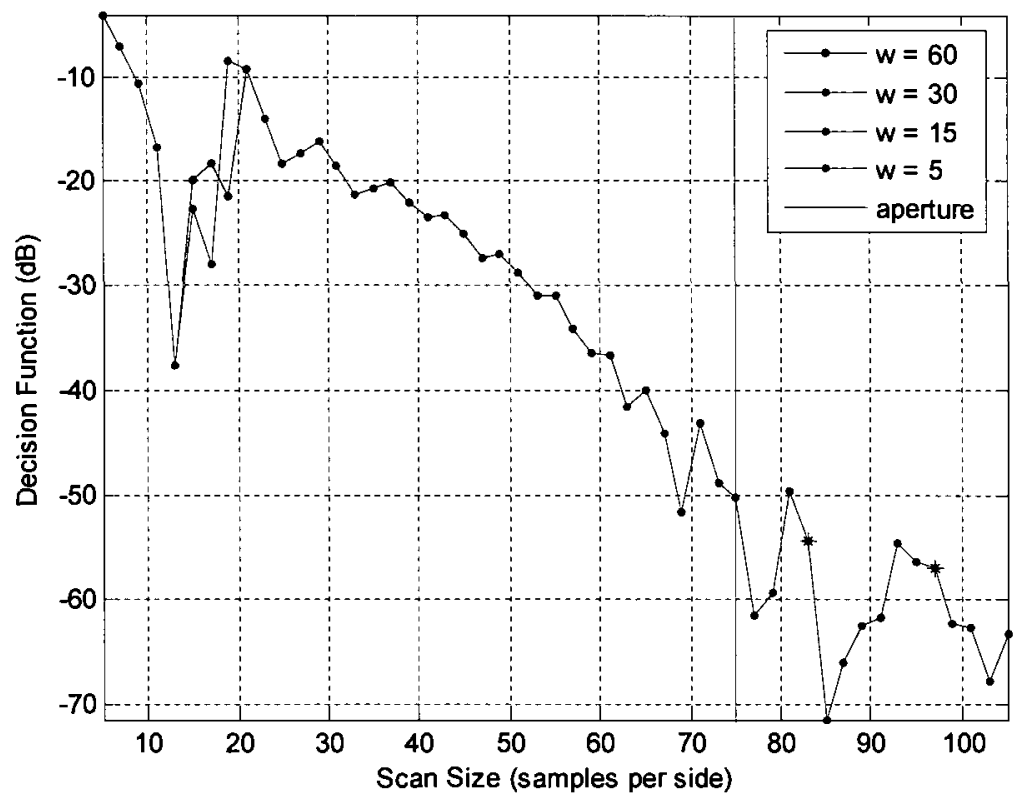

Figure 5.4-13: Co-polarized component of DFE3 for the planar array 


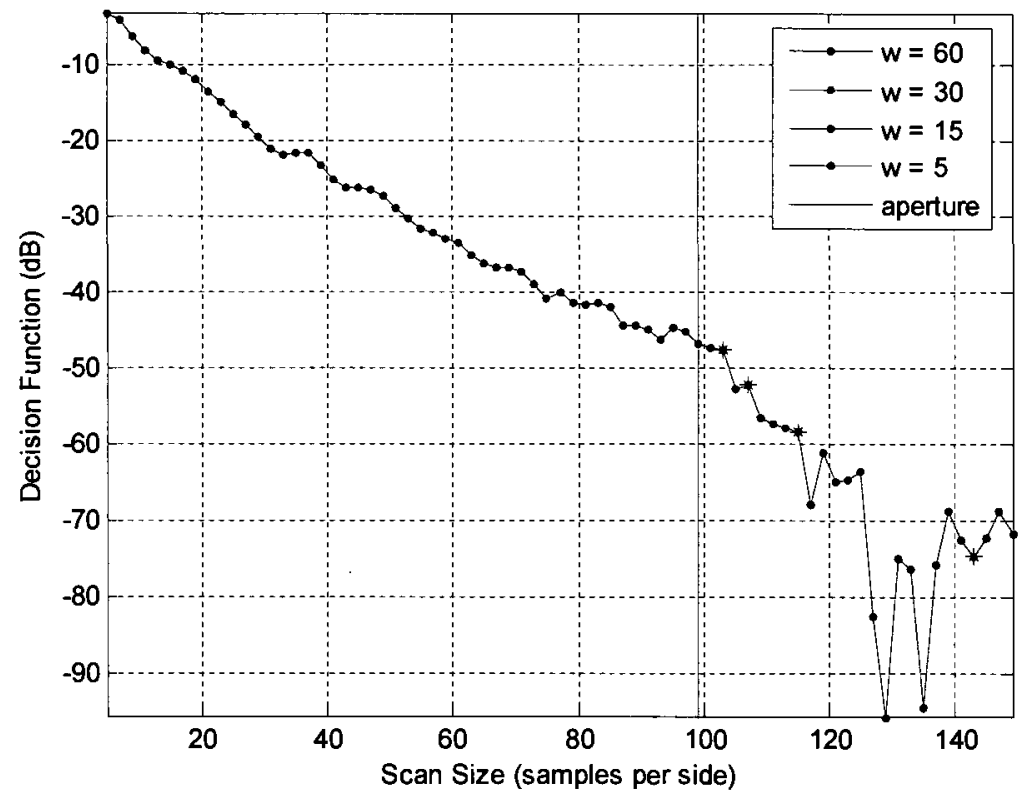

Figure 5.4-14: Co-polarized component of DFE3 for the monopulse reflector antenna

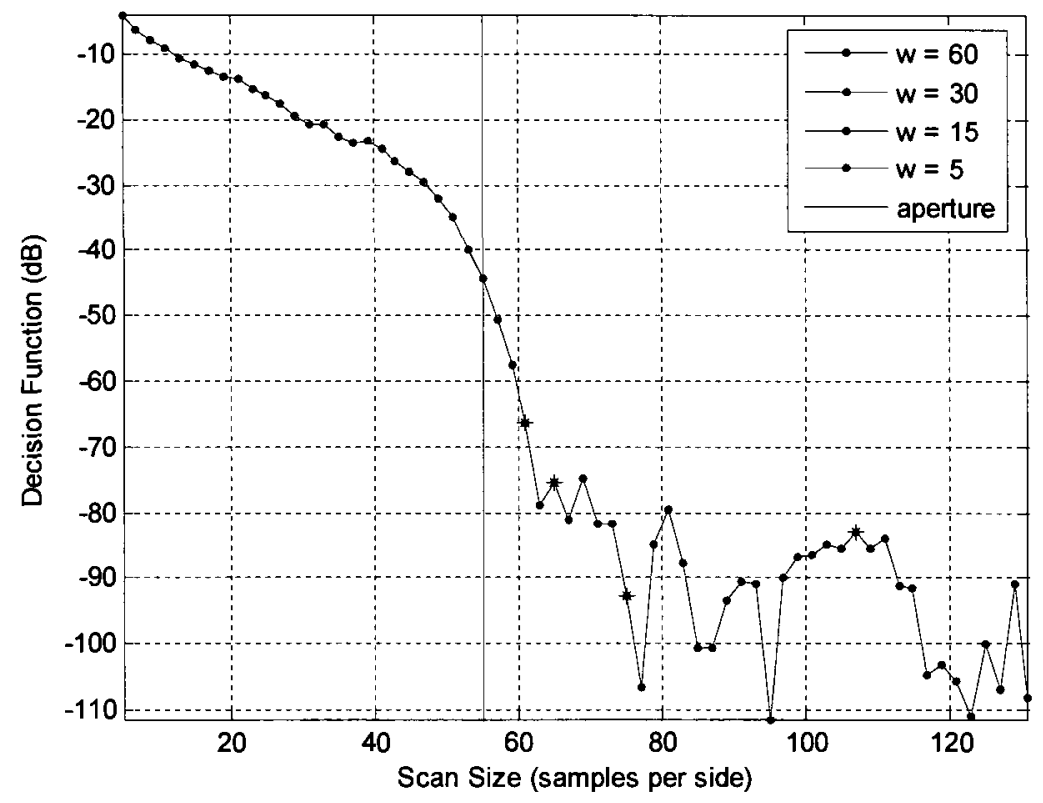

Figure 5.4-15: Co-polarized component of DFE3 for the slotted waveguide array 


\subsection{Analysis of Decision Function DFDn}

\subsubsection{Analysis of Directivity versus Scan Size}

Before discussing the behaviour of the decision functions DFD $n$ we refer back to plots of the maximum directivity shown in Chapter 4 for each of the antennas measured, namely:

- Offset reflector antenna - Figure 4.3-3

- Horn antenna - Figure 4.4-3

- Planar array - Figure 4.5-3

- Monopulse reflector antenna - Figure 4.6-3

- Slotted waveguide array - Figure 4.7-3

Both the co- and cross-polarized maximum directivity plots $^{28}$ converge to some steady value as the scan size increases. At some point the directivity value changes negligibly from the $n^{\text {th }}$ to the $(n-1)^{\text {th }}$ scan. If this change is less than a certain amount, say the accuracy within which the directivity can be determined by the measurement set-up, this can be used to terminate the PNF scan. We notice that for some of the antennas the co-polarized maximum directivity settles down at a smaller scan size than the crosspolarized one does. This is to be expected as the cross-polarized patterns are at much lower levels than the co-polarized ones, and are therefore more sensitive to measurement errors (including truncation error). If the polarization properties are the important

\footnotetext{
${ }^{28}$ These are the values of the maximum directivity. In the case of the co-polarized directivity this occurs in the broadside direction, but in the cross-polarized case the direction in which the directivity is a maximum is not broadside, as for the offset reflector antenna case shown in Figure 4.3-5, for example. As the scan size increases the direction of the directivity maximum may shift around until the scan size is sufficiently large.
} 
properties in some test then both the co- and cross-polarized directivities would need to be tracked.

\subsubsection{Analysis of Decision Function DFD1}

Figure 5.5-1 through Figure 5.5-5 show plots of DFD1 versus scan size for each of the antennas under examination. The quantity DFD1, defined in Section 3.4.4, gives the average of the differences between the directivities in all directions within the region of interest of the $n^{\text {th }}$ and $(n-1)^{\text {th }}$ iteration. The DFD1 value drops off more rapidly with scan size as the region of interest gets smaller. The extent of the difference in the DFD1 behaviour for a particular region of interest size differs from antenna to antenna. It is most distinct for the offset reflector and horn antennas, and least so for the slotted waveguide array. We have not been able to establish why this is so. It is not a function of the electrical size of the AUT (and hence its maximum directivity). Nor does it depend on the distinctiveness of the sidelobes of the AUT (and hence the depth of the pattern nulls). As with DFE1, the decision function DFD1 is not easily related to a quantity in terms of which antenna performance specifications are stated, and so is not that useful in deciding on a scan size truncation point because it is difficult to select a threshold value. 


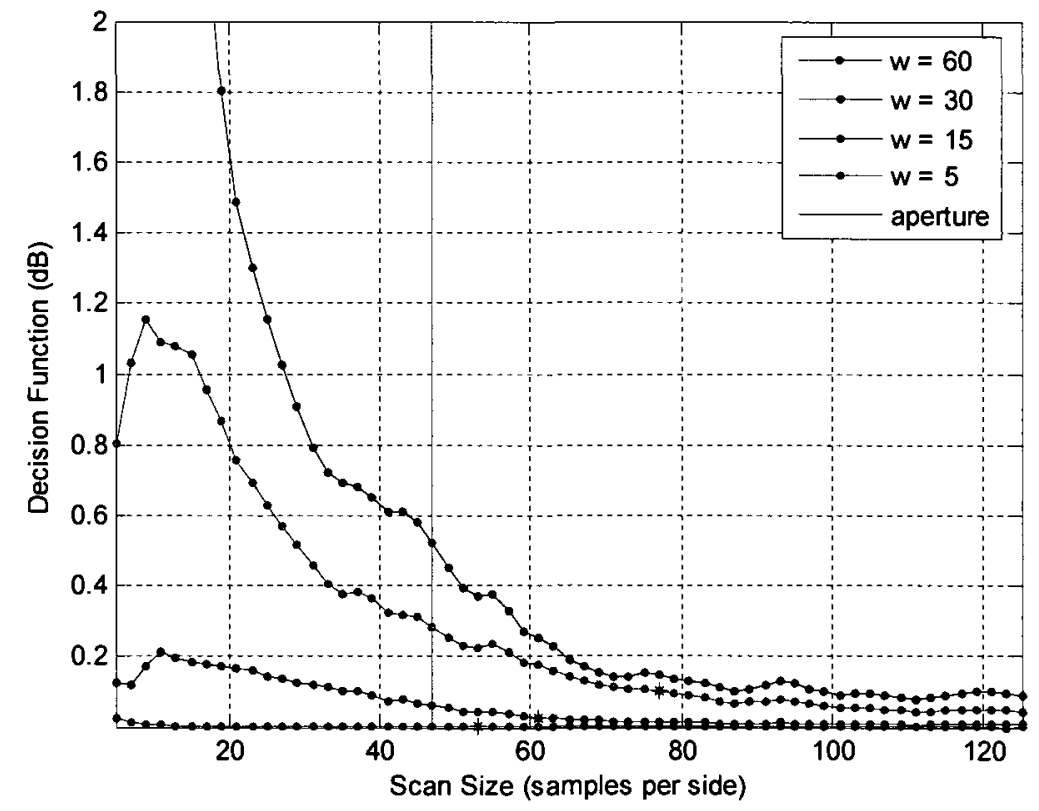

Figure 5.5-1: Co-polarized component of DFD1 for the offset reflector antenna

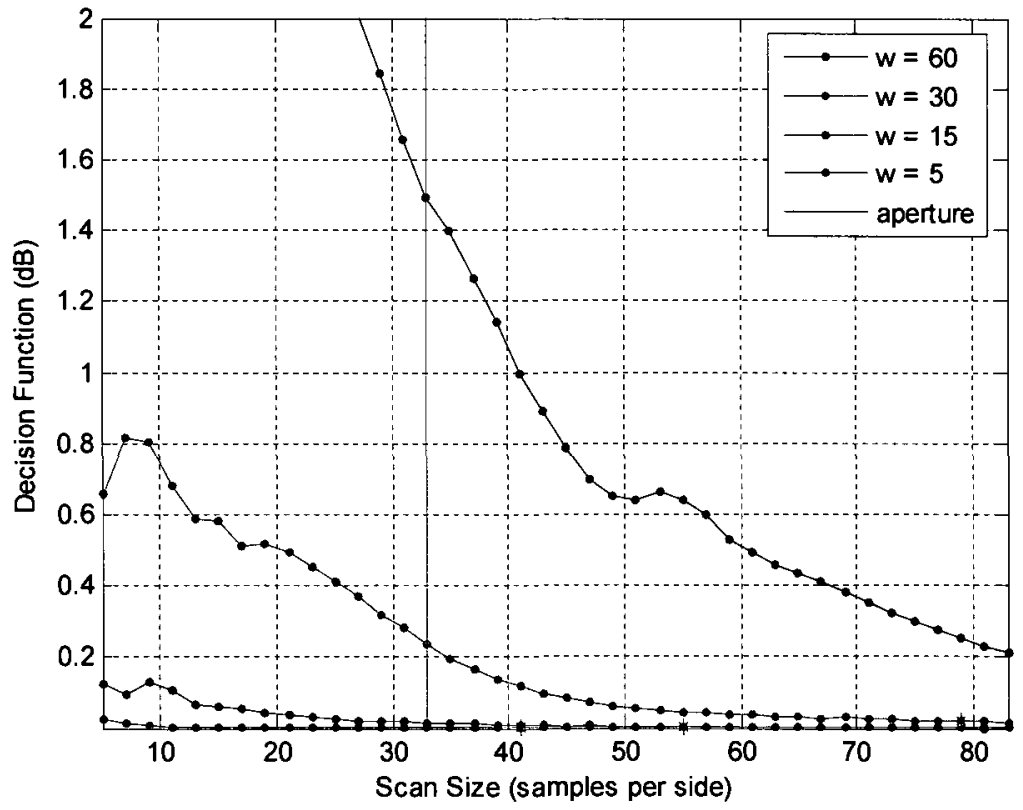

Figure 5.5-2: Co-polarized component of DFD1 for the horn antenna 


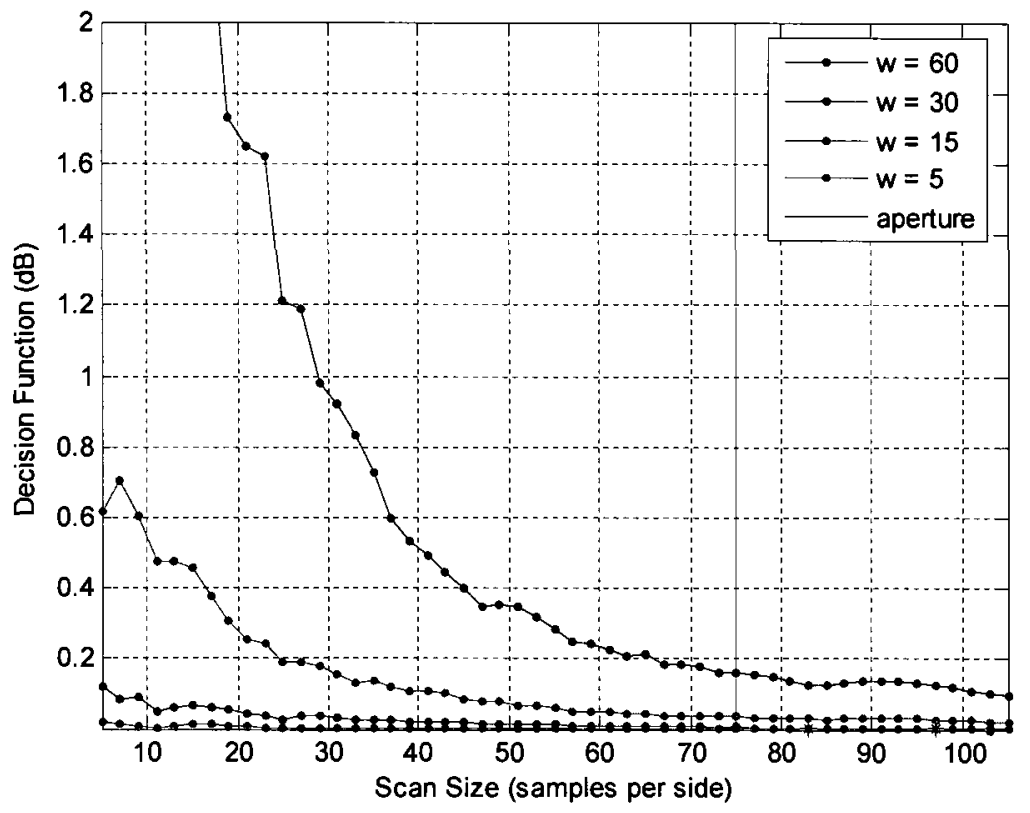

Figure 5.5-3: Co-polarized component of DFD1 for the planar array

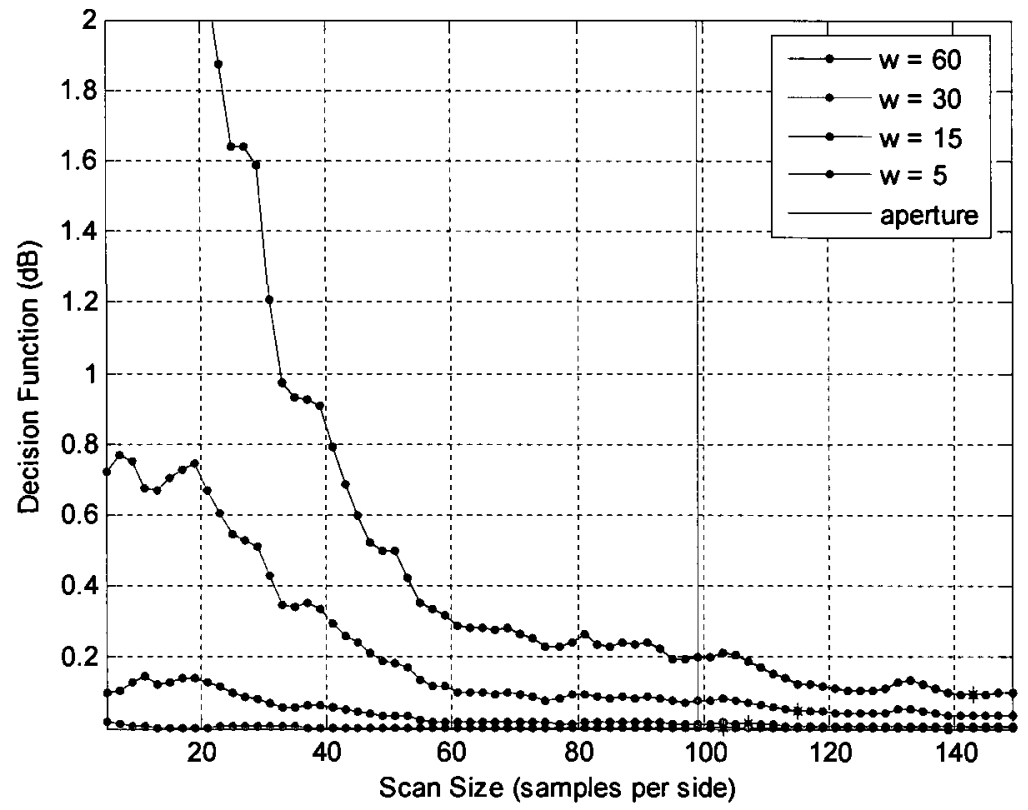

Figure 5.5-4: Co-polarized component of DFD1 for the monopulse reflector antenna 


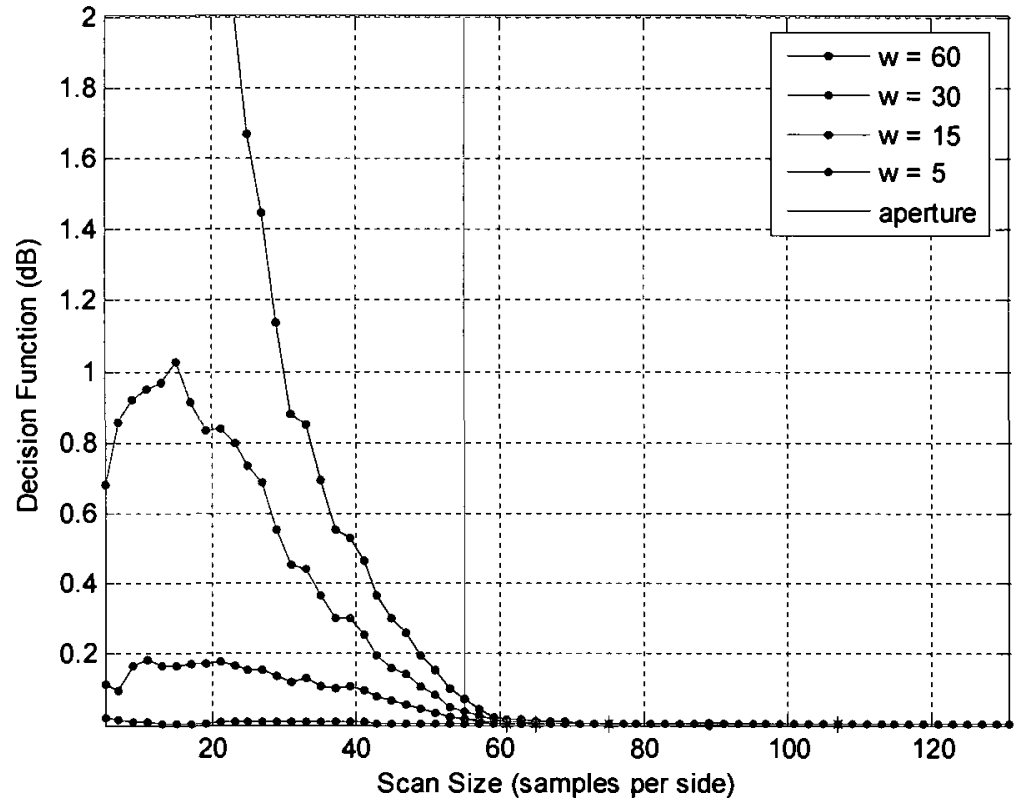

Figure 5.5-5: Co-polarized component of DFD1 for the slotted waveguide array

\subsubsection{Analysis of Decision Function DFD2}

Figure 5.5-6 through Figure 5.5-10 show plots of DFD2 versus scan size for each of the five AUTs. As with DFD1, the quantity DFD2, defined in Section 3.4.5, computes the difference between the directivities after the $n^{\text {th }}$ and $(n-1)^{\text {th }}$ scan in all directions within the region of interest. However, unlike DFD1 it does not average these values but picks out the largest difference, which is not necessarily in the direction of maximum directivity. The direction in which this maximum directivity difference occurs changes as the scan size increases and depends on the extent of the region of interest. The reason is that this decision function may sample the directivity in the vicinity of the pattern dips where the directivity has a low value, which values are extremely sensitive to small changes in scan size and even computational issues. This is evidenced in the fact that the 
plots of DFD2 are relatively erratic, and in fact become increasingly so if the dynamic range of the data considered in the DFD2 determination is widened by altering the value of quantity $\mathrm{P}_{\mathrm{DFD}, \mathrm{co}}$ and $\mathrm{P}_{\mathrm{DFD}, \text { cr }}$ from $-50 \mathrm{~dB}$ to $-100 \mathrm{~dB}^{29}$.

We are in fact not too concerned with precise values of the directivity in the pattern dips; it is the directivity in the main beam and largest sidelobe direction that are the most important performance measures. Thus we conclude that DFD2 is not a preferred decision function. Instead of using all observation directions in the region of interest to compute a decision function we instead examine the differences in directivity (obtained for the current and previous scan sizes) in selected key directions ${ }^{30}$ that are directly related to the antenna pattern specifications. This leads to DFD3.

29 So we can mitigate the worst of these effects by adjusting the value of $P_{D F D, c o}$, and $\mathrm{P}_{\mathrm{DFD}, \mathrm{cr}}$.

${ }^{30}$ For instance the direction of maximum directivity and the directivity in the direction of the largest sidelobes. 


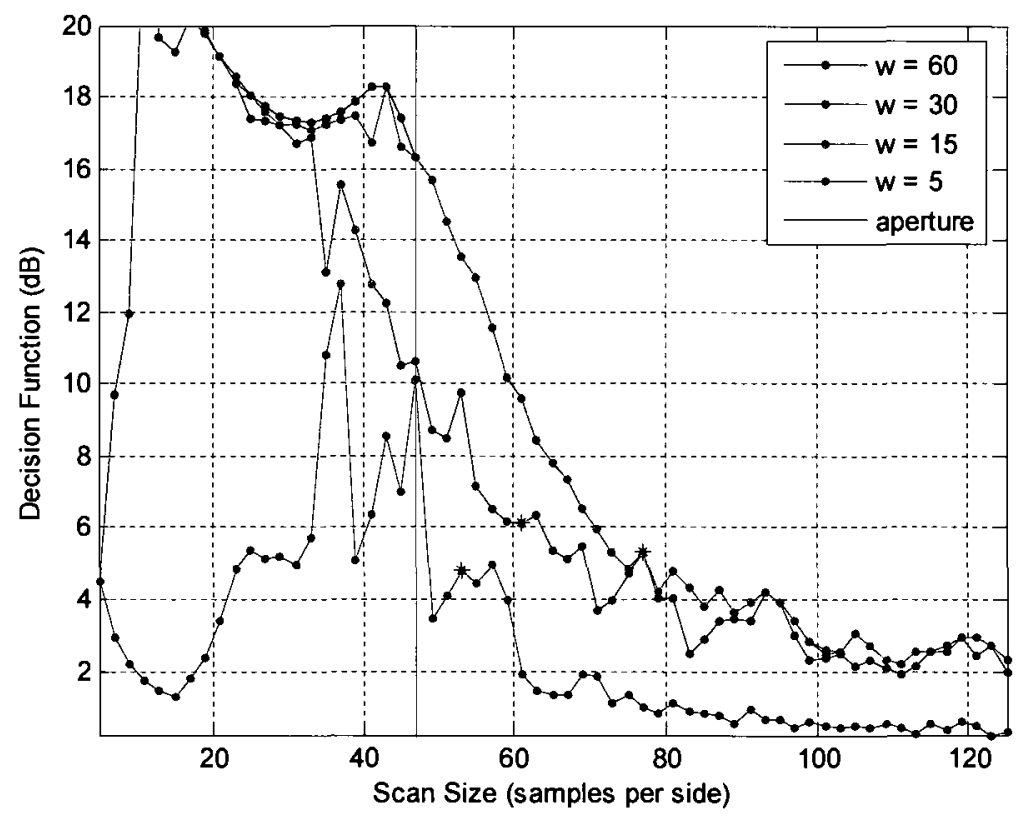

Figure 5.5-6: Co-polarized component of DFD2 for the offset reflector antenna

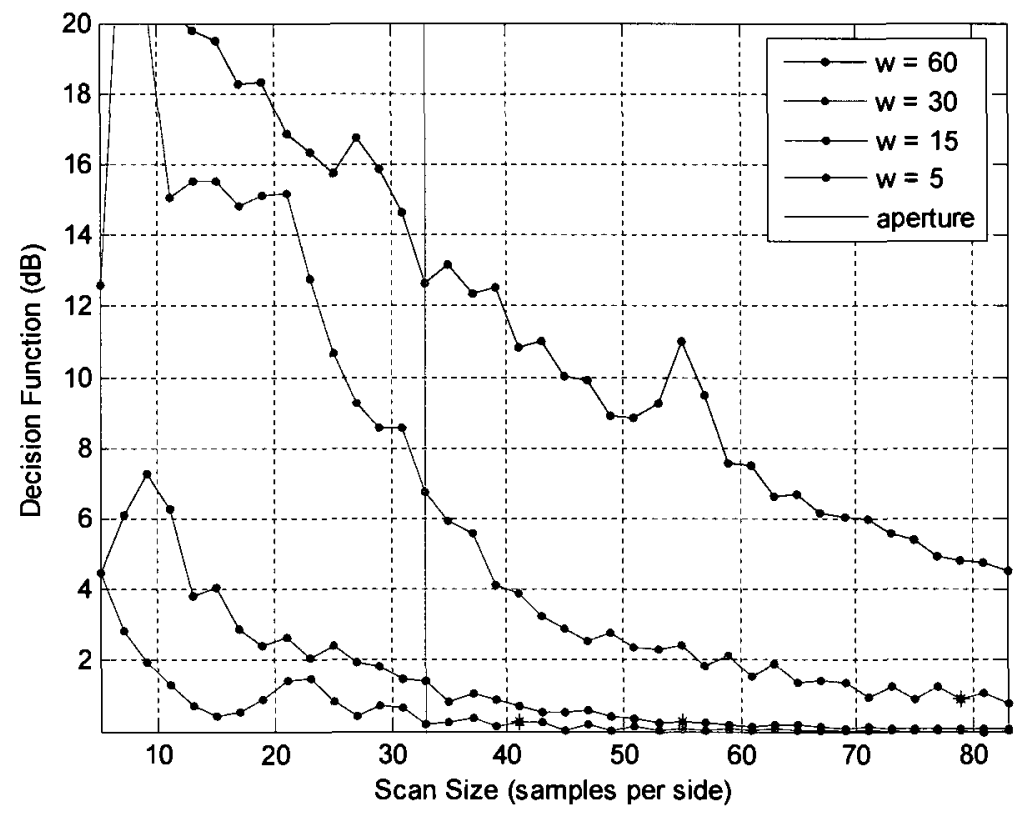

Figure 5.5-7: Co-polarized component of DFD2 for the horn antenna 


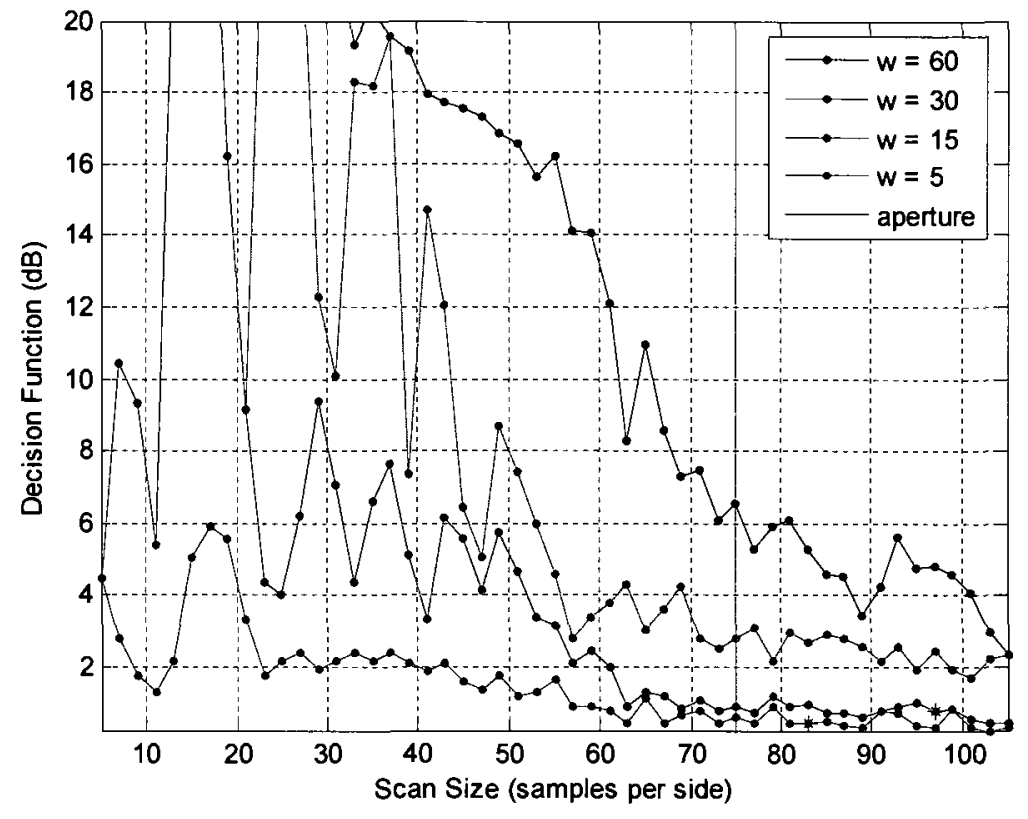

Figure 5.5-8: Co-polarized component of DFD2 for the planar array

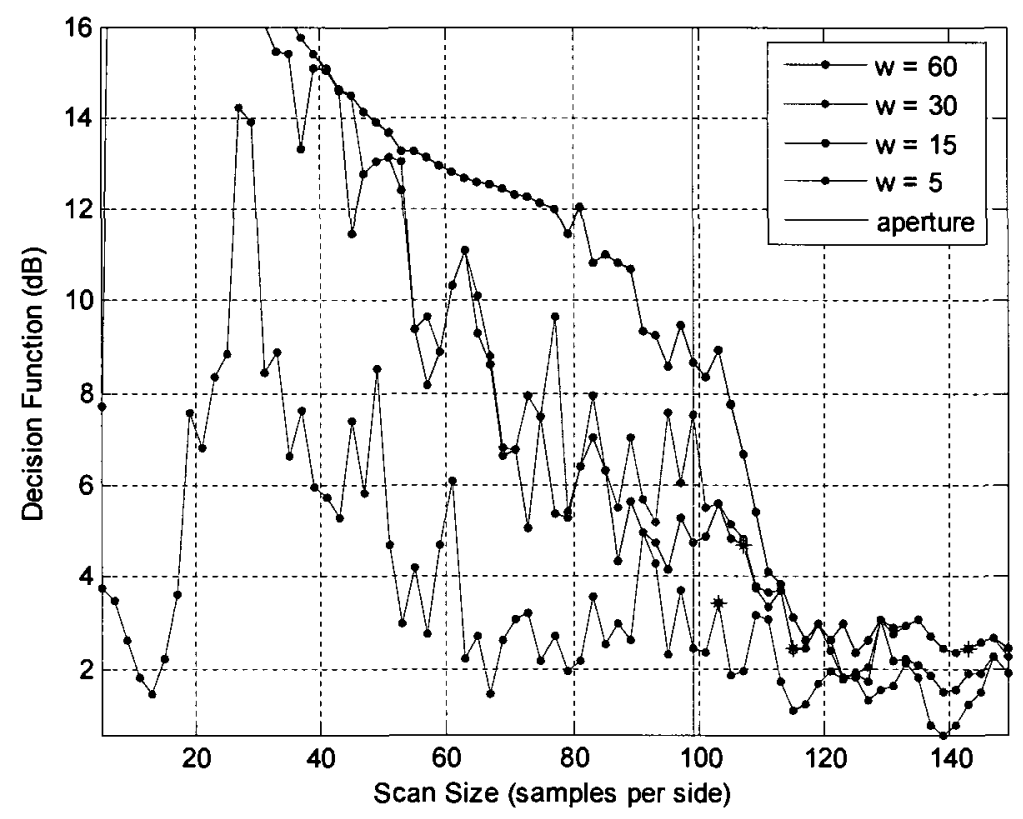

Figure 5.5-9: Co-polarized component of DFD2 for the monopulse reflector antenna 


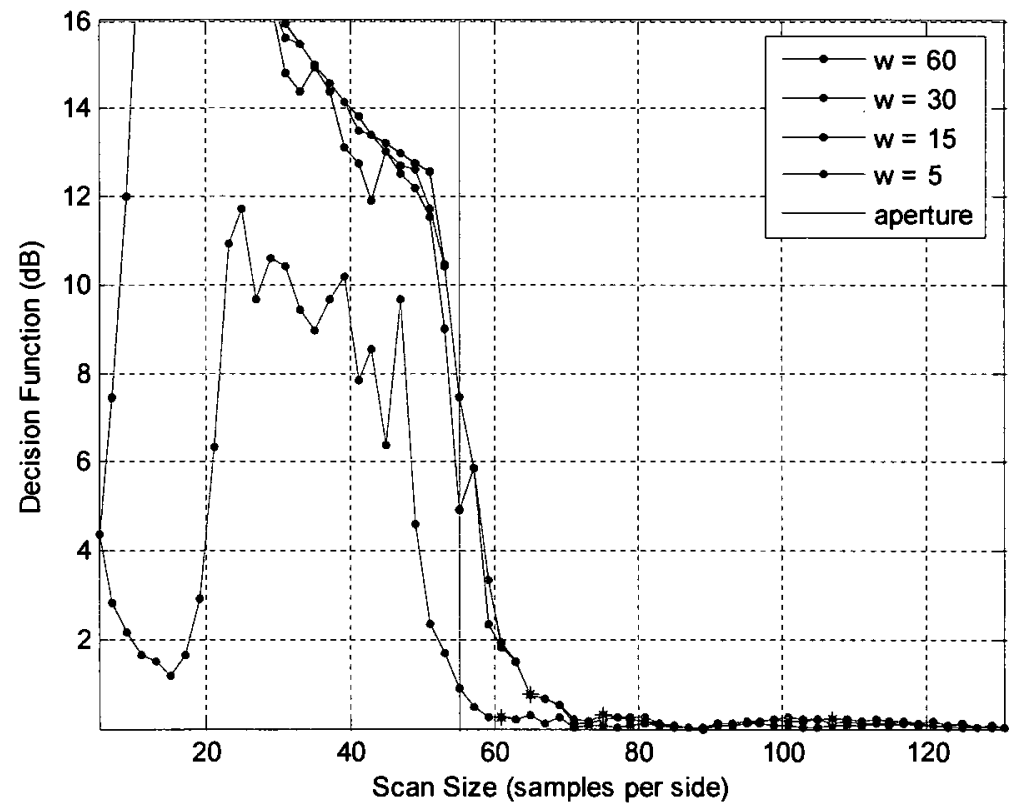

Figure 5.5-10: Co-polarized component of DFD2 for the slotted waveguide array

\subsubsection{Analysis of Decision Function DFD3}

Figure 5.5-11 through Figure 5.5-15 show plots of DFD3 versus scan size for each of the AUTs under examination. In particular, the selected observation direction used here is that of the directivity maximum ${ }^{31}$ for each AUT. We note that the directivity differences are computed here (as they were in fact done for DFD1 and DFD2) by subtracting the $\mathrm{dBi}$ values of the directivity. The reason was that we want the value of DFD3 to decrease to zero as the scan size increases. Once DFD3 is different form zero by less than the measurement accuracy of the PNF range in use, the PNF scans can be terminated.

\footnotetext{
${ }^{31}$ But we could use several key directions and observe them collectively.
} 


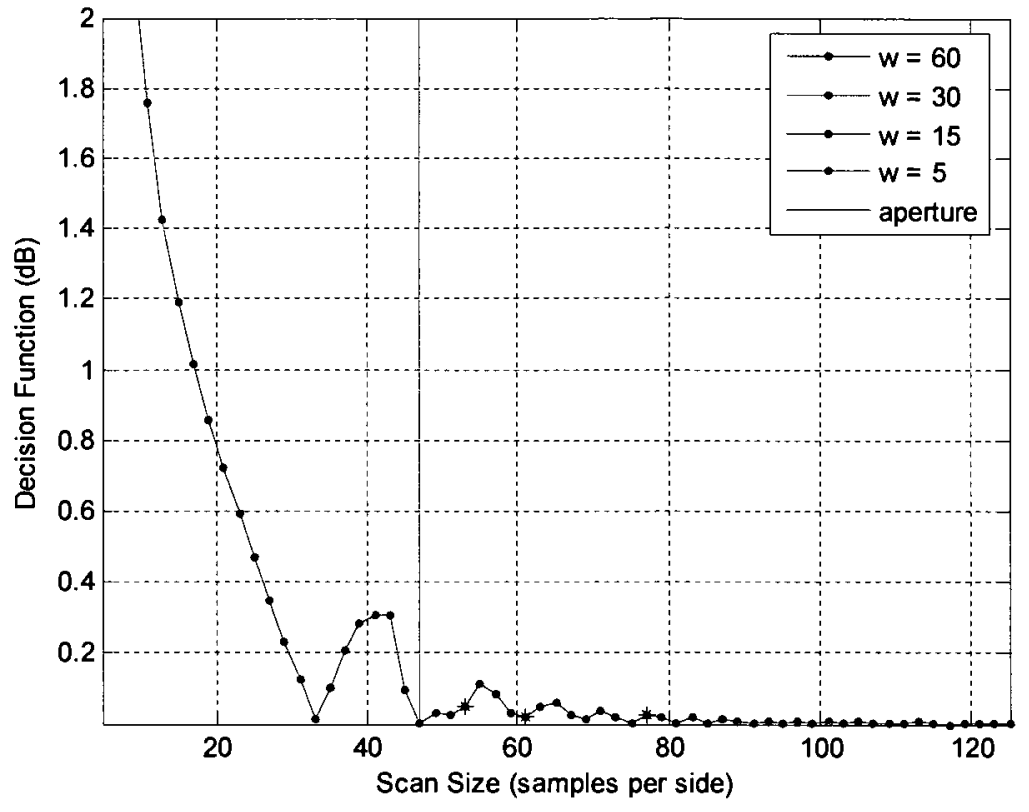

Figure 5.5-11: Co-polarized component of DFD3 for the offset reflector antenna

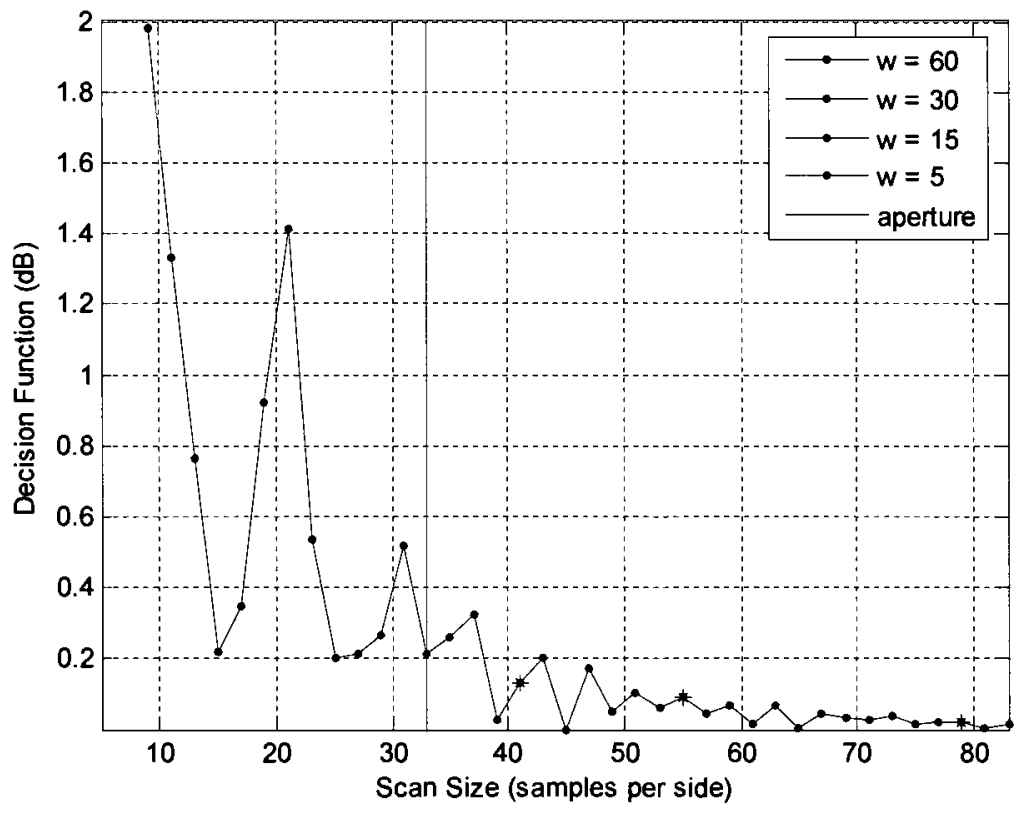

Figure 5.5-12: Co-polarized component of DFD3 for the horn antenna 


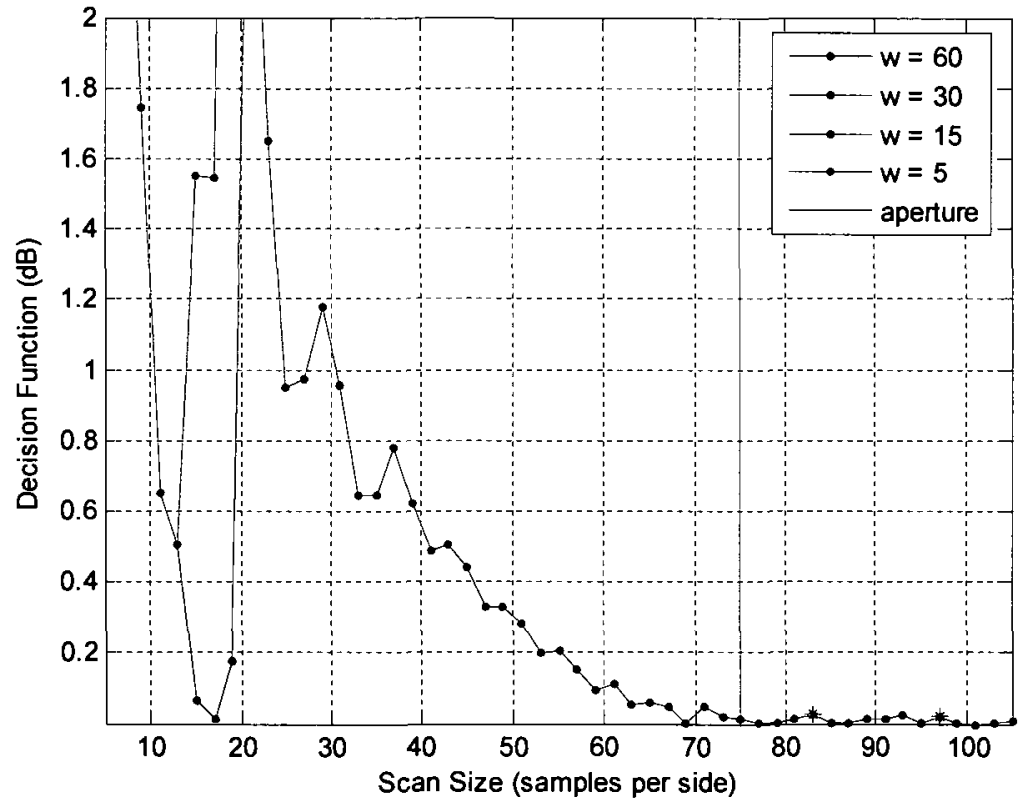

Figure 5.5-13: Co-polarized component of DFD3 for the planar array

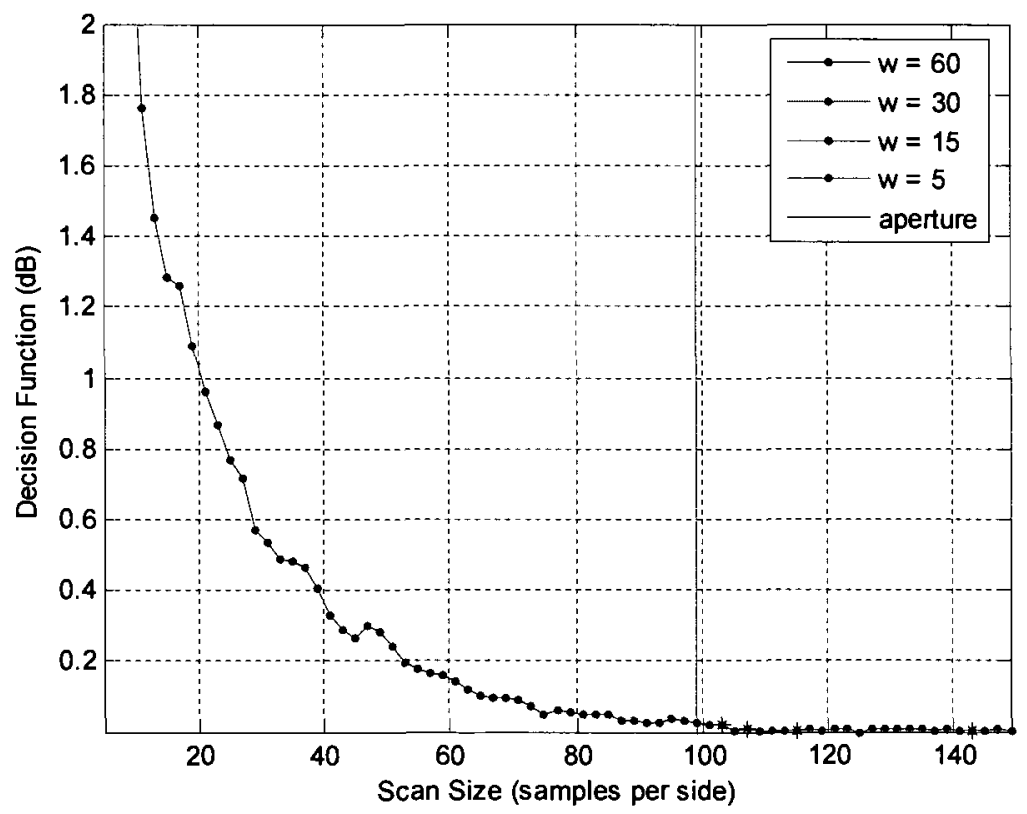

Figure 5.5-14: Co-polarized component of DFD3 for the monopulse reflector antenna 


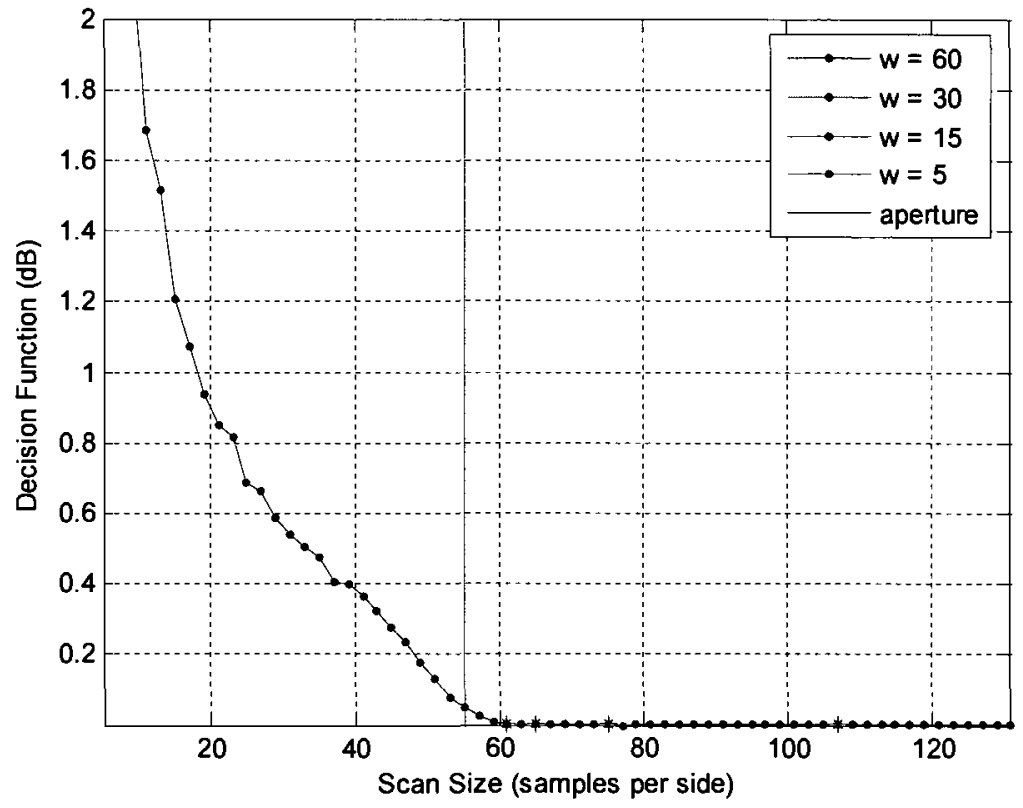

Figure 5.5-15: Co-polarized component of DFD3 for the slotted waveguide array

\subsection{Analysis of Decision Functions DFNn}

The analysis of the DFNn decision functions presented in this section will focus only on the principle near-field component of the antennas, either the $E_{x}$ or $E_{y}$ component. Table 5.6-1 summarizes the principle near-field components for the antenna types. 
Table 5.6-1: Summary of principle near-field components for antenna types

\begin{tabular}{|l|l|}
\hline \multicolumn{1}{|c|}{ AUT } & Principal Near-Field Component \\
\hline Offset Reflector Antenna & $E_{y}$ \\
\hline Horn Antenna & $E_{x}$ \\
\hline Planar Array & $E_{y}$ \\
\hline Monopulse Reflector Antenna & $E_{y}$ \\
\hline Slotted Waveguide Array & $E_{x}$ \\
\hline
\end{tabular}

\subsubsection{Analysis of Decision Function DFN1}

Expressions for the computation of the fractional plane wave spectrum error, and its relation to the uncertainty in the directivity in any direction, were discussed in Section 3.4.7. It is clear from the presented discussion what value of plane wave spectrum fractional error is needed for a required uncertainty in the directivity in any direction; in other words how to translate the fractional error in the plane wave spectrum to a directivity uncertainty number. DFN1 averages this fractional error quantity over the entire region of interest. It is shown in Figure 5.6-1 through Figure 5.6-5. As expected, the smaller the region of interest the lower the fractional error for a given scan size. Unfortunately DFN1 suffers from the same difficulty (namely an inability to select a threshold) as the other averaging decision functions DFE1 and DFD1. 


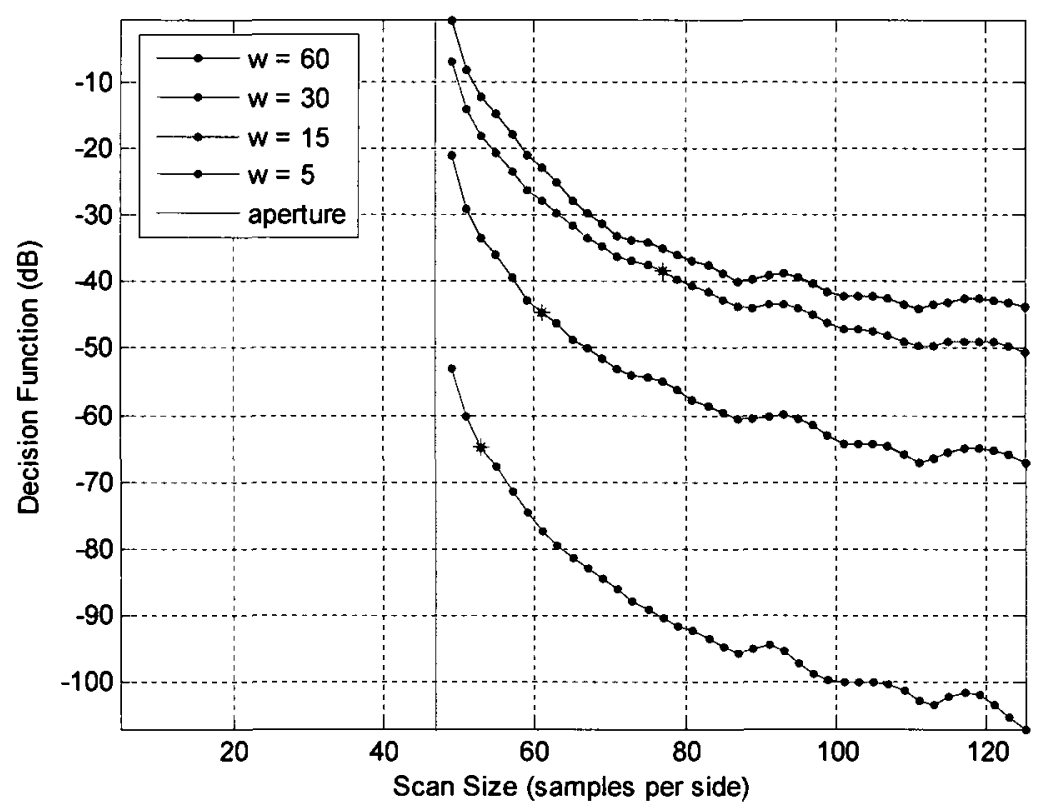

Figure 5.6-1: Y-component of DFN1 for the offset reflector antenna

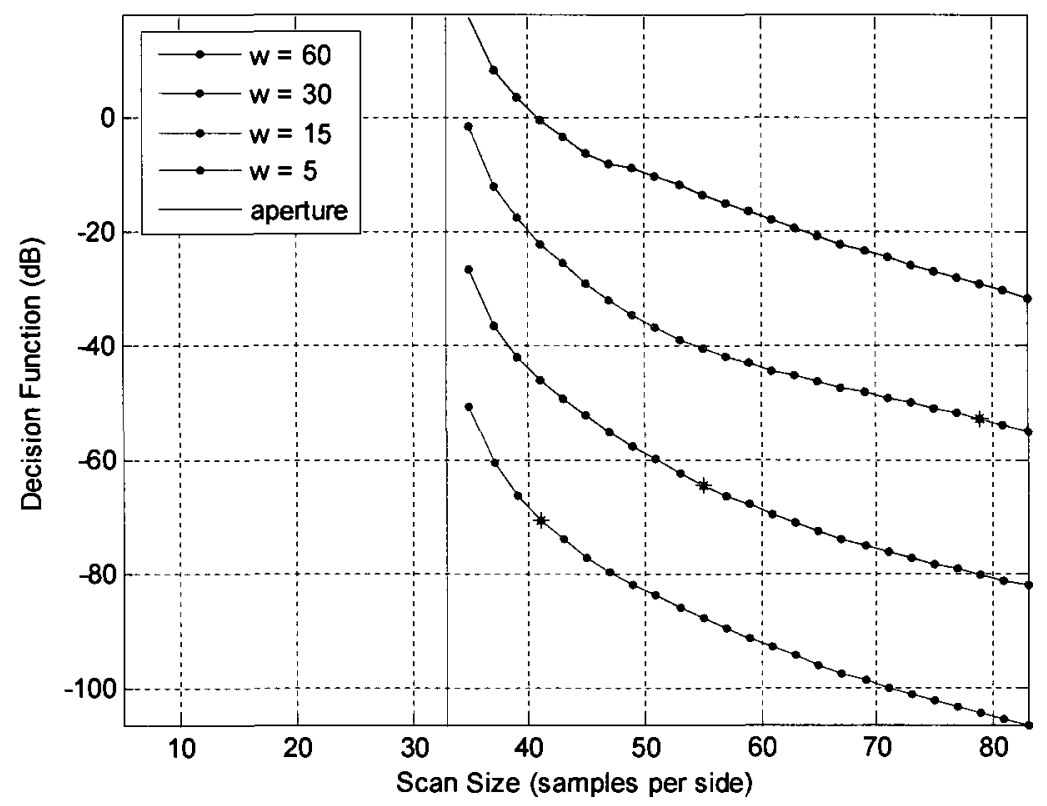

Figure 5.6-2: X-component of DFN1 for the horn antenna 


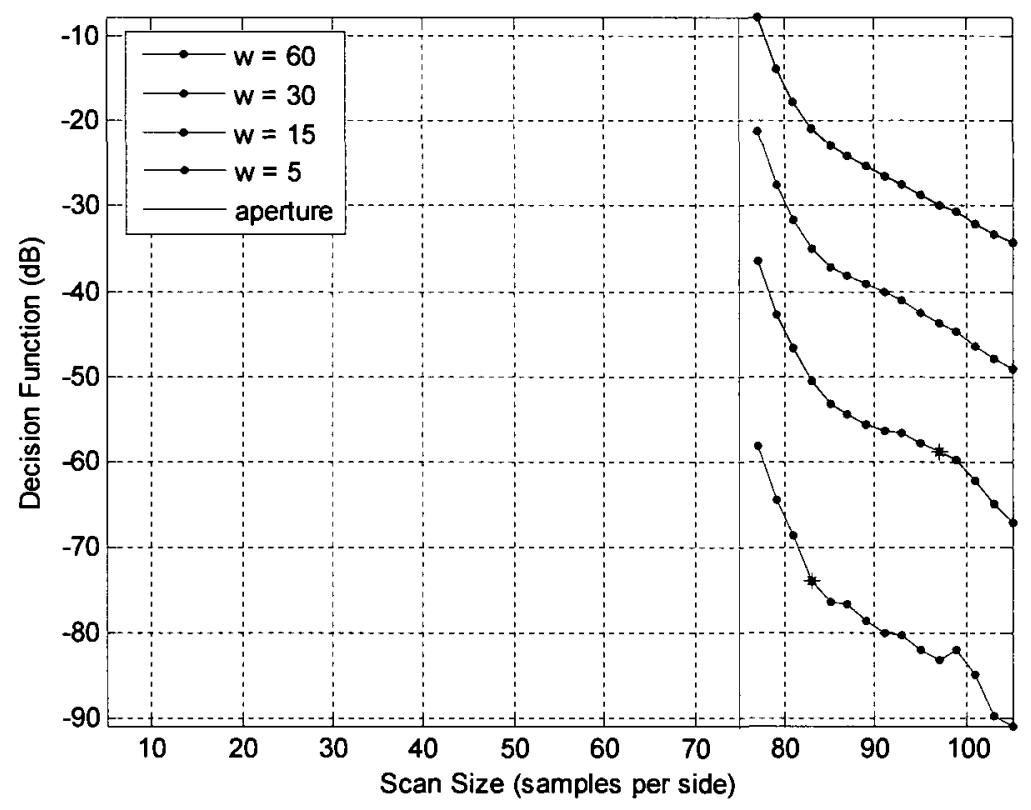

Figure 5.6-3: Y-component of DFN1 for the planar array

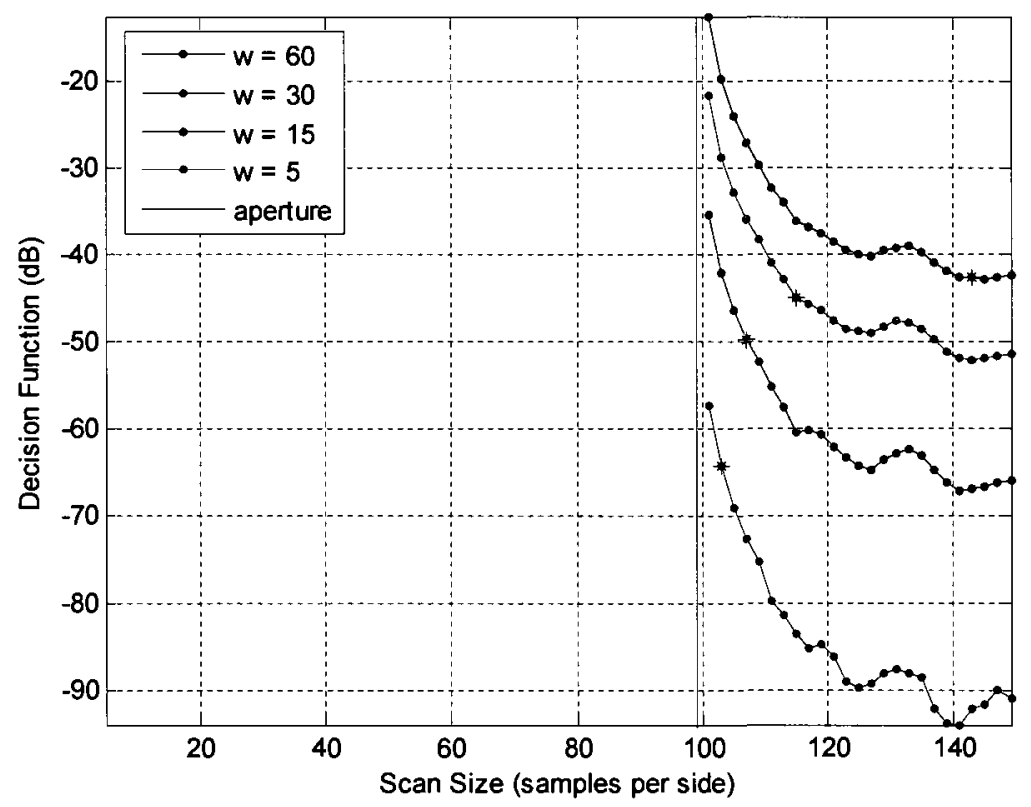

Figure 5.6-4: Y-component of DFN1 for the monopulse reflector antenna 


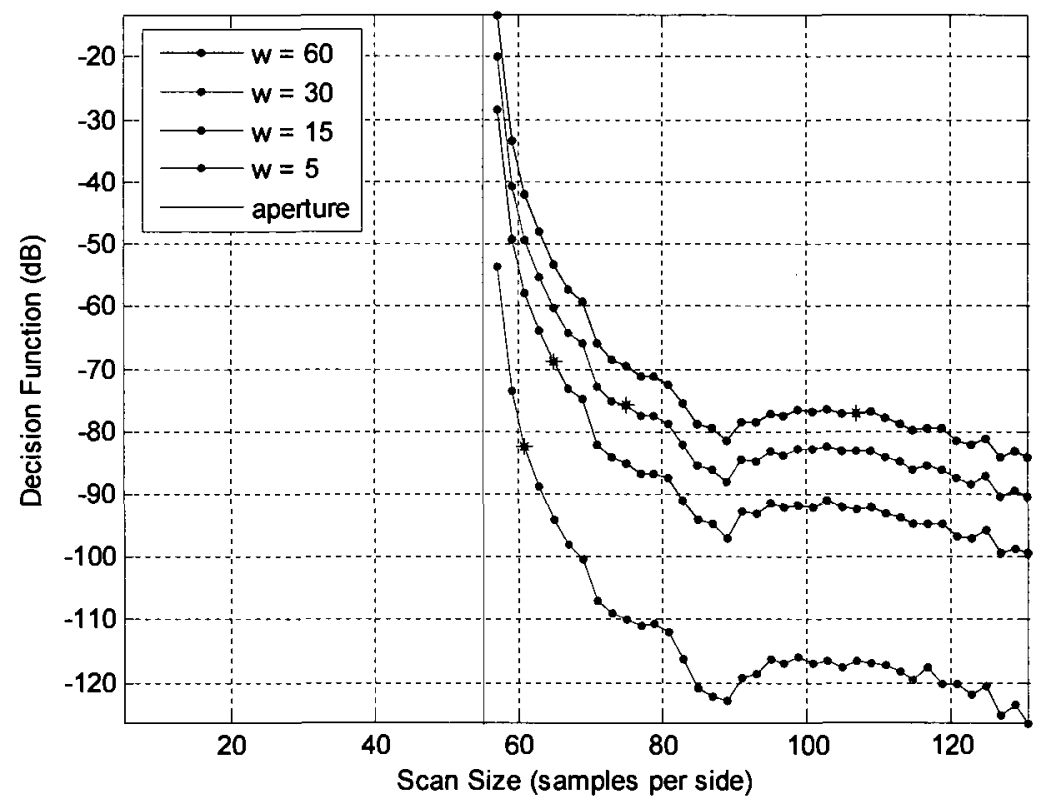

Figure 5.6-5: X-component of DFN1 for the slotted waveguide array

\subsubsection{Analysis of Decision Function DFN2}

Decision function DFN2, given in Figure 5.6-6 through Figure 5.6-10, shows the maximum value of the fractional error of the plane wave spectrum in the indicated region of interest. Like DFE2 and DFD2 it does not decrease monotonically but has an envelope below which the plot falls. In spite of the non-monotonicity, DFN2 is a useful decision function due to the fact that it possible to relate its numerical value directly to the uncertainty in directivity over the region of interest, and so can be used to take a sound decision on when to terminate the PNF scan. Care must be taken, however, as the surface integral in the denominator of expressions (3.4.7-1) and (3.4.7-2) can contain nulls which will cause very large errors in the DFN2. These can be mitigated by adjusting the values of $P_{D F N, x}$, and $P_{D F N, y}$ to not include these low values in the evaluation of DFN2. These 
nulls were typically found in the larger angles off broadside, and are why the plots for the different $\zeta$ are sometimes large. An investigation into this behaviour is presented in Appendix B.

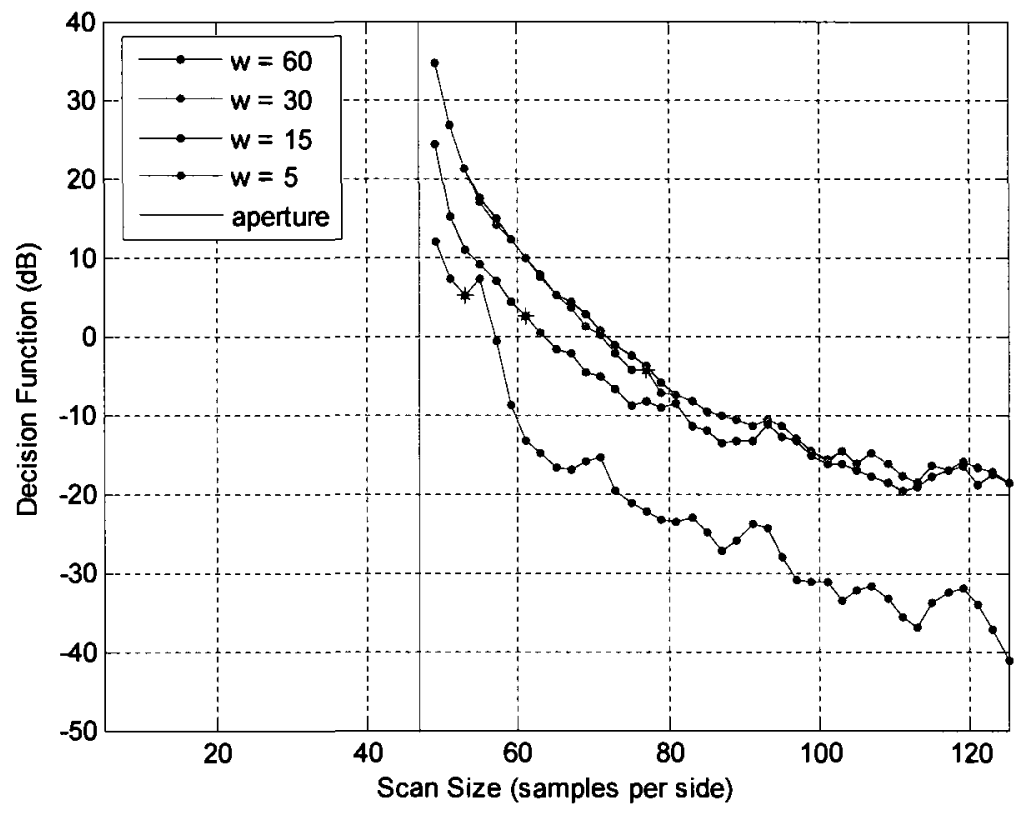

Figure 5.6-6: Y-component of DFN2 for the offset reflector antenna 


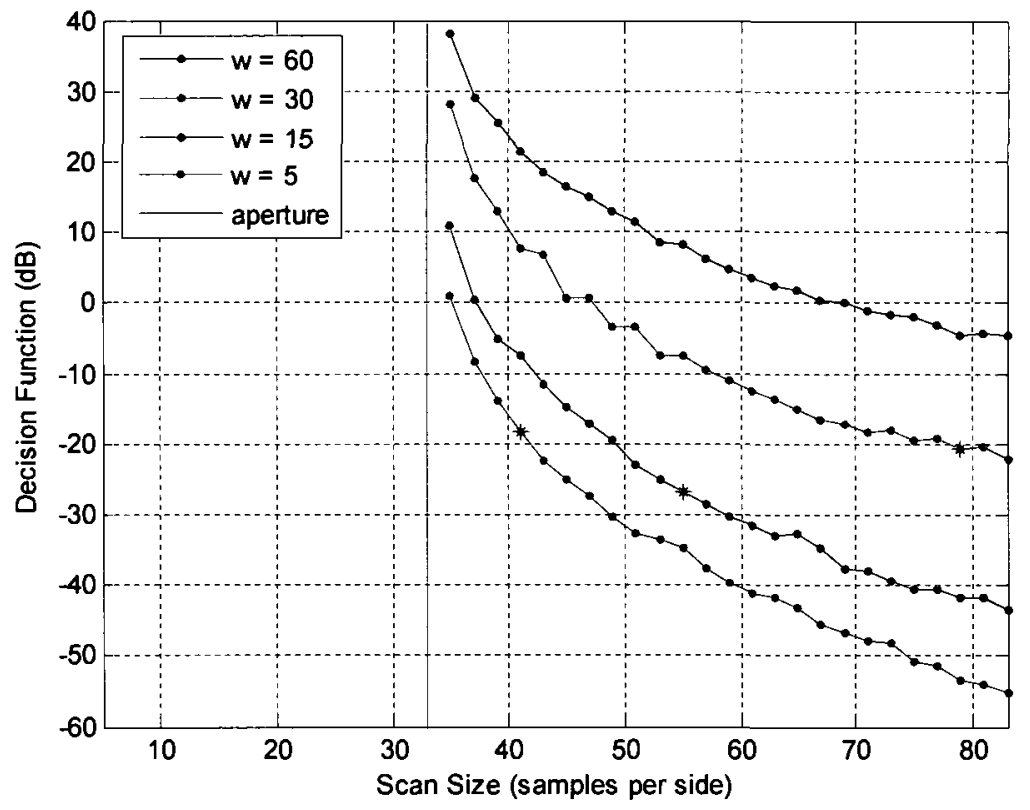

Figure 5.6-7: X-component of DFN2 for the horn antenna

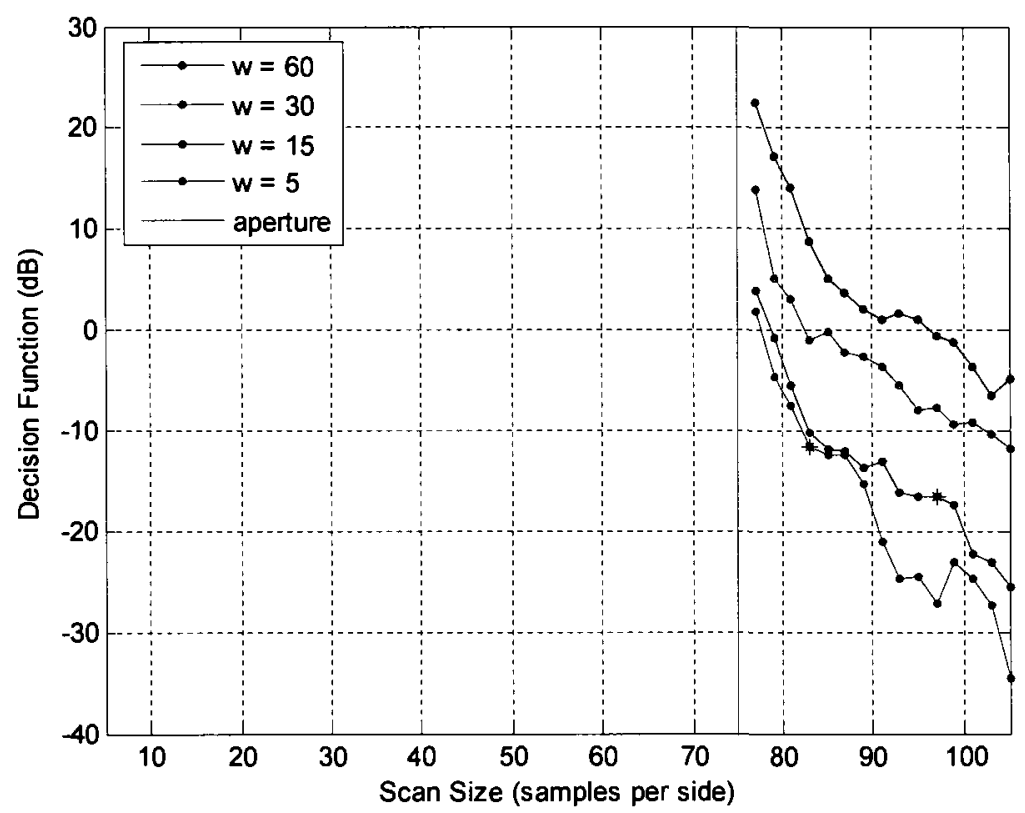

Figure 5.6-8: Y-component of DFN2 for the planar array 


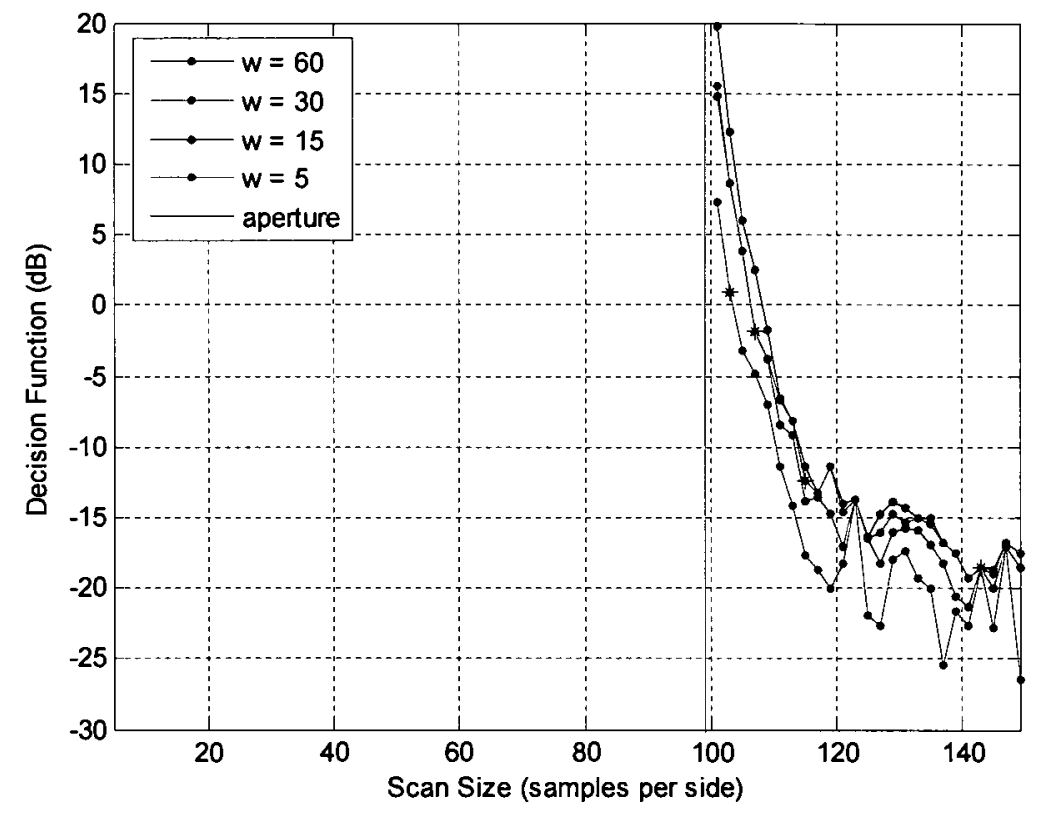

Figure 5.6-9: Y-component of DFN2 for the monopulse reflector antenna

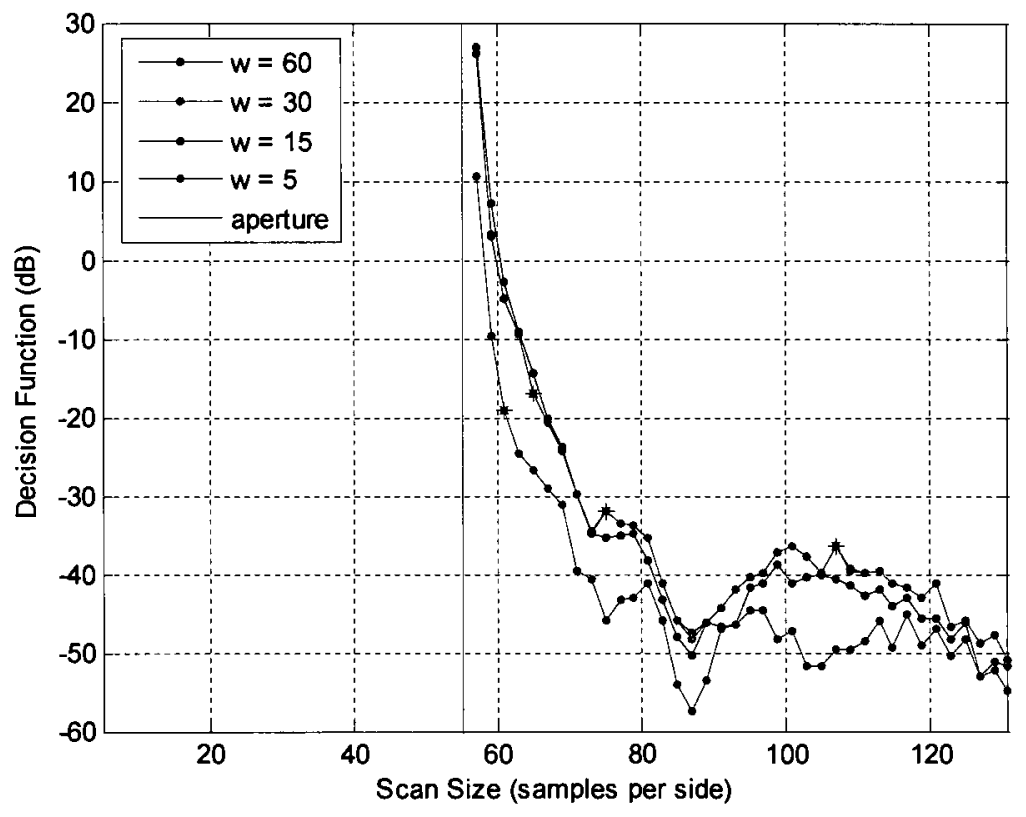

Figure 5.6-10: X-component of DFN2 for the slotted waveguide array 


\subsubsection{Analysis of Decision Function DFN3}

Decision function DFN3 differs from DFN2 in that it is the fractional error in the plane wave spectrum in a specified direction. Figure 5.6-11 through Figure 5.6-15 show plots of DFN3 versus scan size for the AUTs under consideration when the selected observation direction used is that in the direction of the pattern maximum ${ }^{32}$. These all decrease with increasing scan size. The advantage in using decision function DFN3 is that (from Section 3.4.7) it is possible to relate its numerical value directly to the uncertainty in directivity in the direction of the main beam, in the direction of significant sidelobes, or any other direction. Although not discussed in Section 3.4.7, it is also possible to relate it to an uncertainty in the measured bandwidth [1].

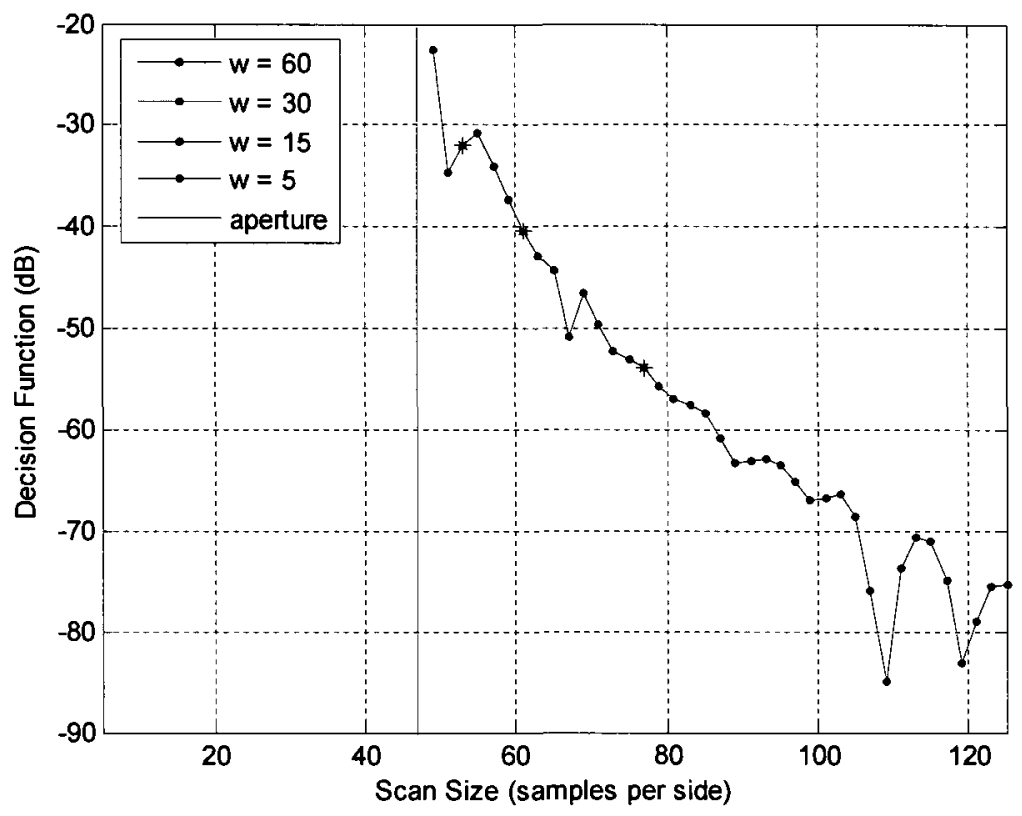

Figure 5.6-11: Y-component of DFN3 for the offset reflector antenna

\footnotetext{
${ }^{32}$ Because the pattern maxima are within a $5^{\circ}$ cone for all the AUTs under discussion the plots of DFN3 are independent of the region of interest sizes shown in the inset on the plots.
} 


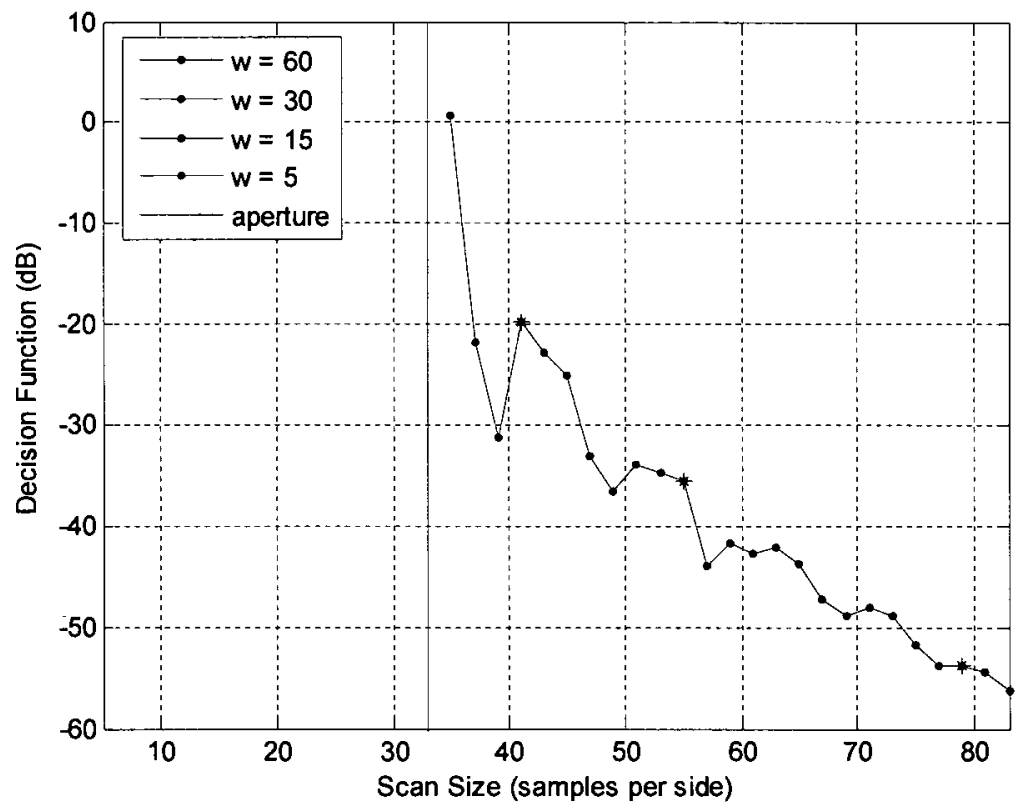

Figure 5.6-12: X-component of DFN3 for the horn antenna

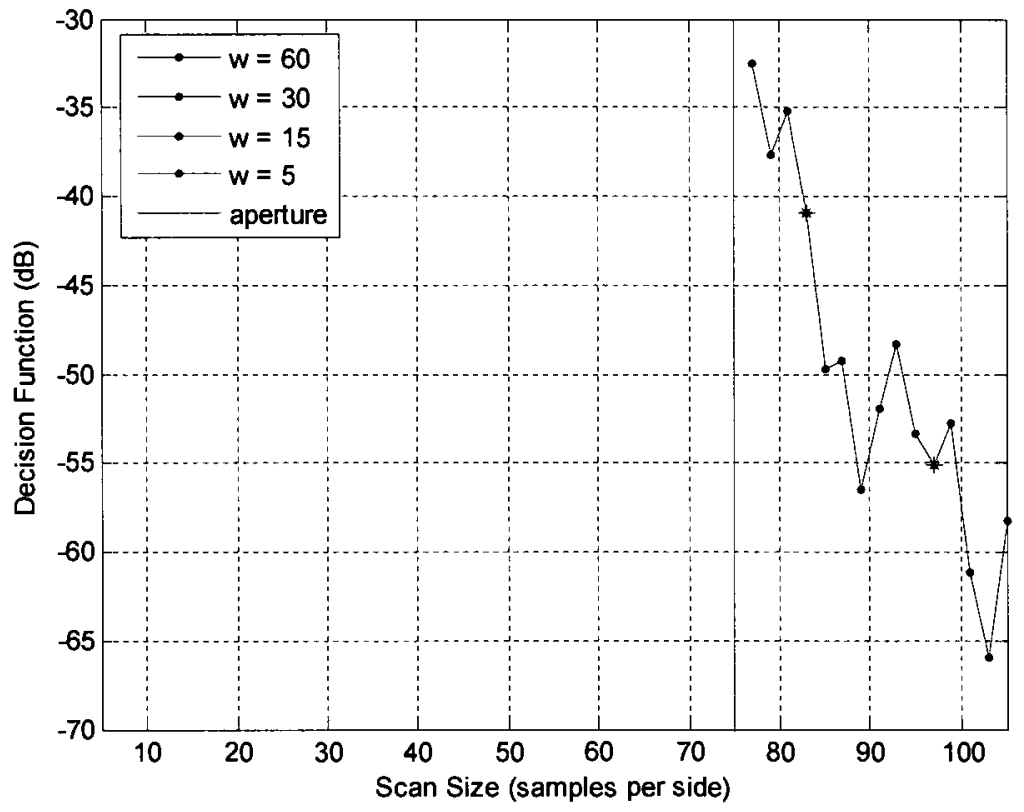

Figure 5.6-13: Y-component of DFN3 for the planar array 


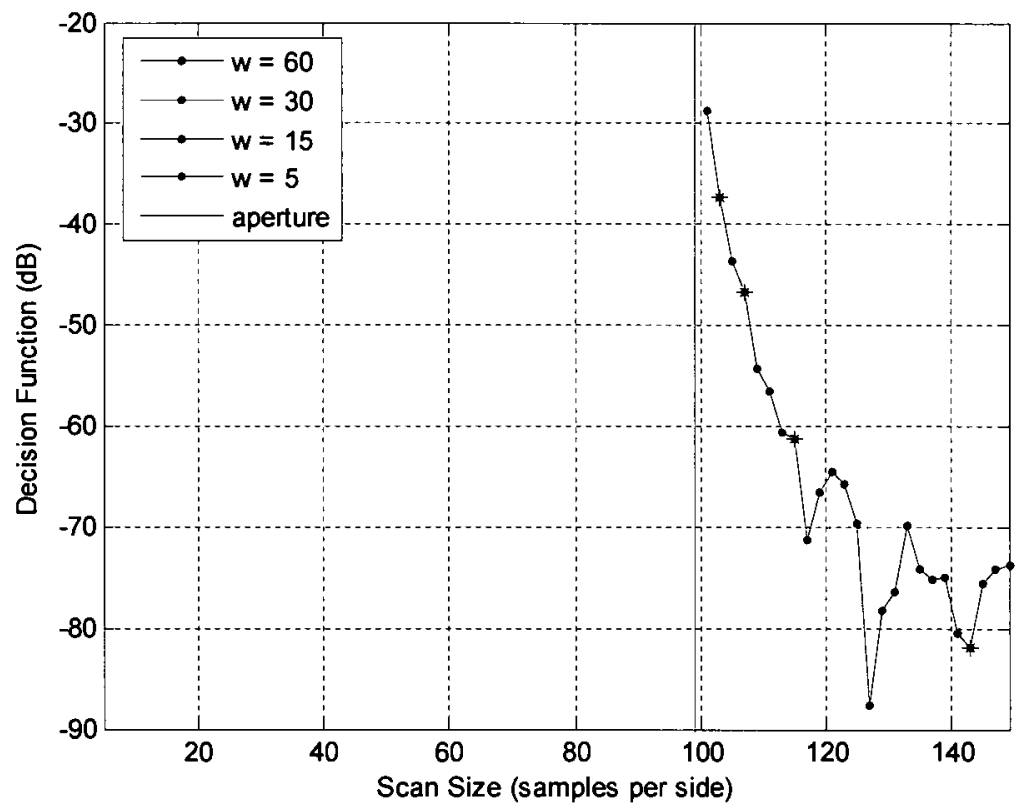

Figure 5.6-14: Y-component of DFN3 for the monopulse reflector antenna

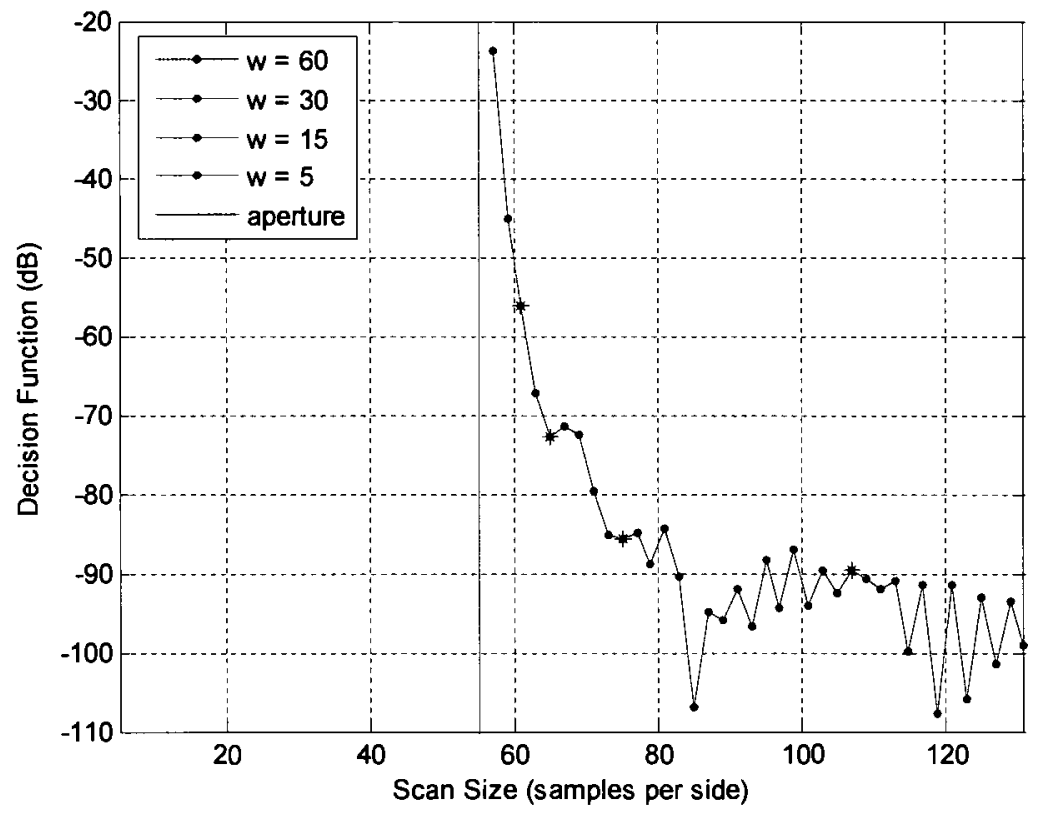

Figure 5.6-15: X-component of DFN3 for the slotted waveguide array 


\subsection{Analysis of Cross-Polarized Decision Functions}

Although the co-polarized components of the decision functions are of primary interest, it is prudent to show the analysis of the cross-polarized component of the decision functions. Therefore, presented in this section for the offset reflector antenna which has the highest cross-polarized component of the antennas presented in this thesis. The evaluation of the cross-polarized decision functions reach the same conclusions as the co-polarized cases, and these will not be repeated here.

\subsubsection{Analysis of Cross-Polarized Decision Function DFEn}

The cross-polarized DFEn are lower than the co-polarized components, which was also the case for the other antennas. DFE1, DFE2, and DFE3 also level off at a PNF scan size of 83 samples per side. The maximum error, DFE2, falls below $-50 \mathrm{~dB}$ at a PNF scan size of 51 samples per side, and from Table 3.4-1 this relates to an uncertainty of $\pm 0.5 \mathrm{~dB}$ at a pattern level of $-25 \mathrm{~dB}$. 


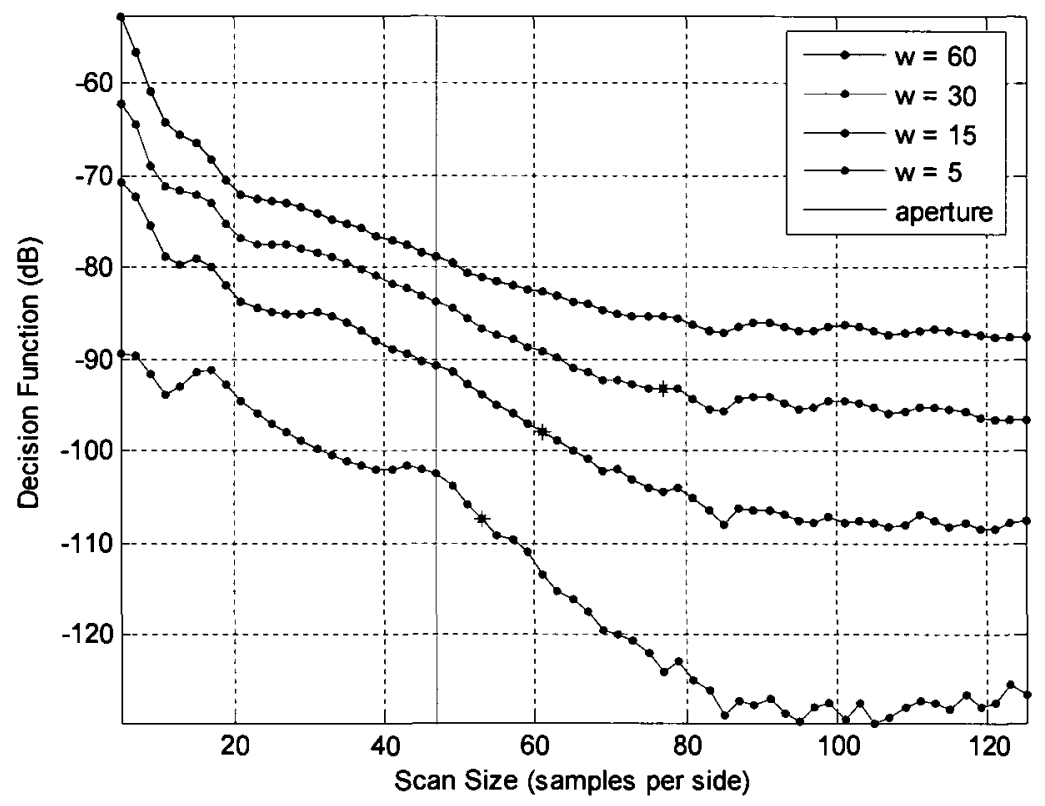

Figure 5.7-1: Cross-polarized component of DFE1 for the offset reflector antenna

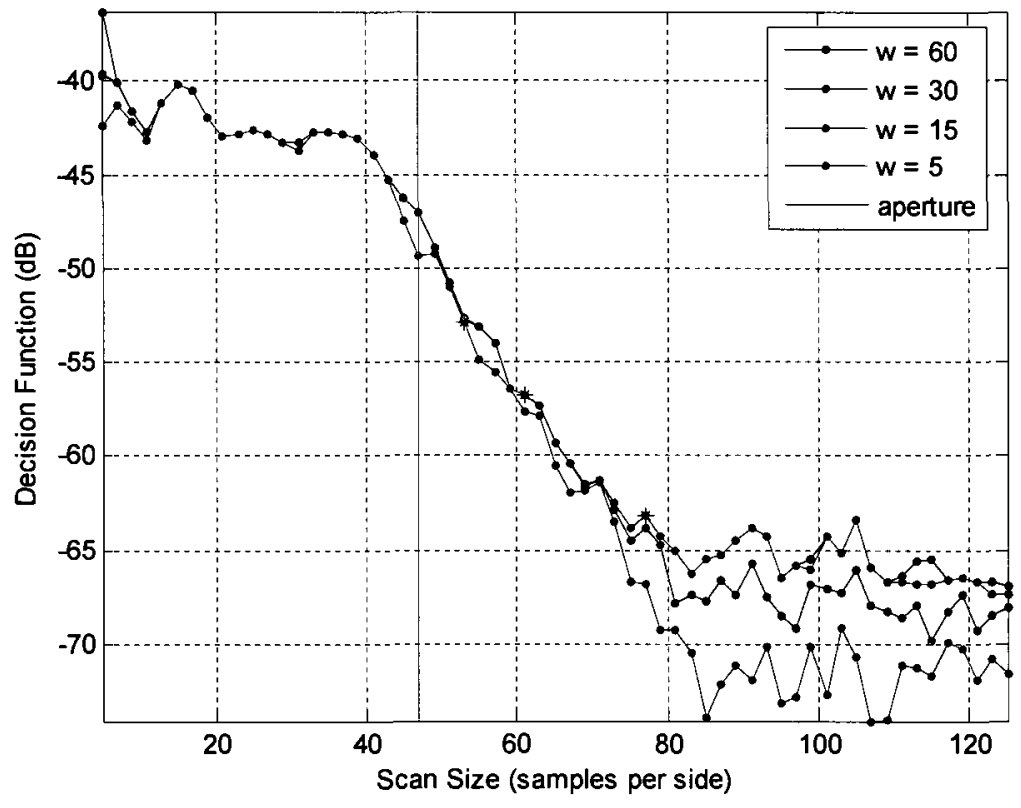

Figure 5.7-2: Cross-polarized component of DFE2 for the offset reflector antenna 


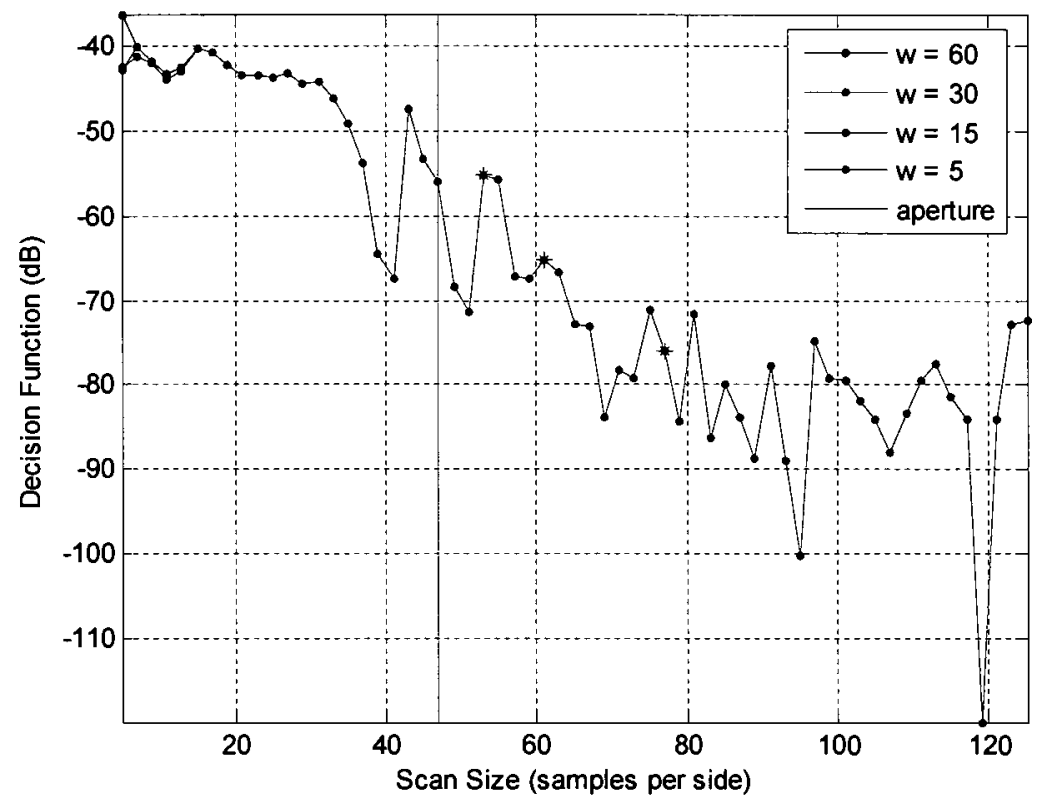

Figure 5.7-3: Cross-polarized component of DFE3 for the offset reflector antenna

\subsubsection{Analysis of Cross-Polarized Decision Function DFDn}

The cross-polarized DFD1 is also smaller, although the largest difference is seen when $\xi=60^{\circ}$. For the other $\xi$ there is only a slight difference between the co- and crosspolarized DFD1. The cross-polarized DFD2 exhibits more noise than the co-polarized DFD2, although the value does drop significantly shortly after the aperture has been scanned. The cross-polarized DFD3 is slightly larger than the co-polarized decision function, although the overall shape of the two plots are very similar. The DFD3 for the cross-polarized case was evaluated at the maximum directivity of the cross-polarized directivity. 


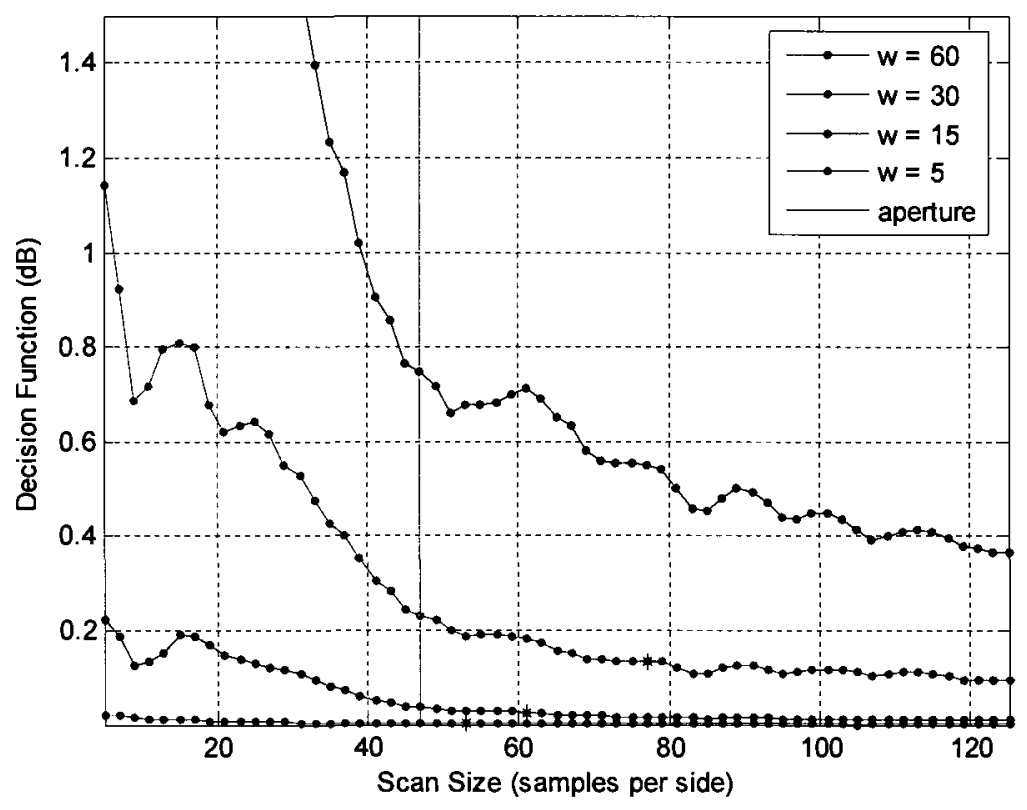

Figure 5.7-4: Cross-polarized component of DFD1 for the offset reflector antenna

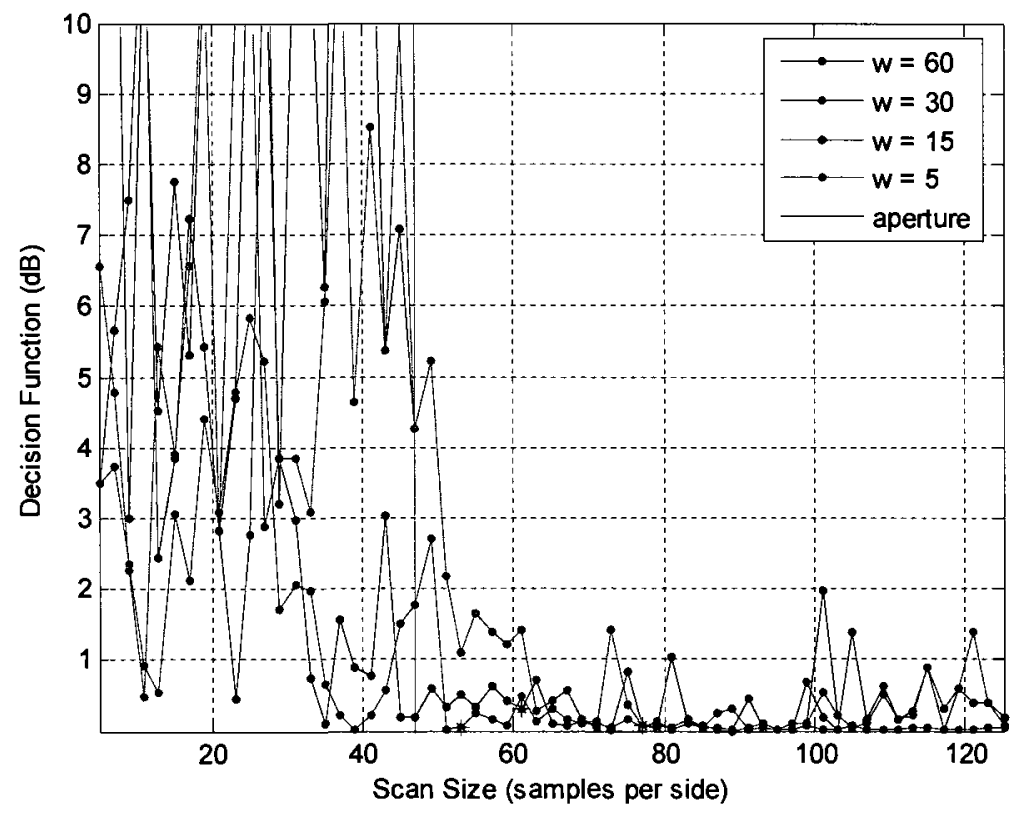

Figure 5.7-5: Cross-polarized component of DFD2 for the offset reflector antenna 


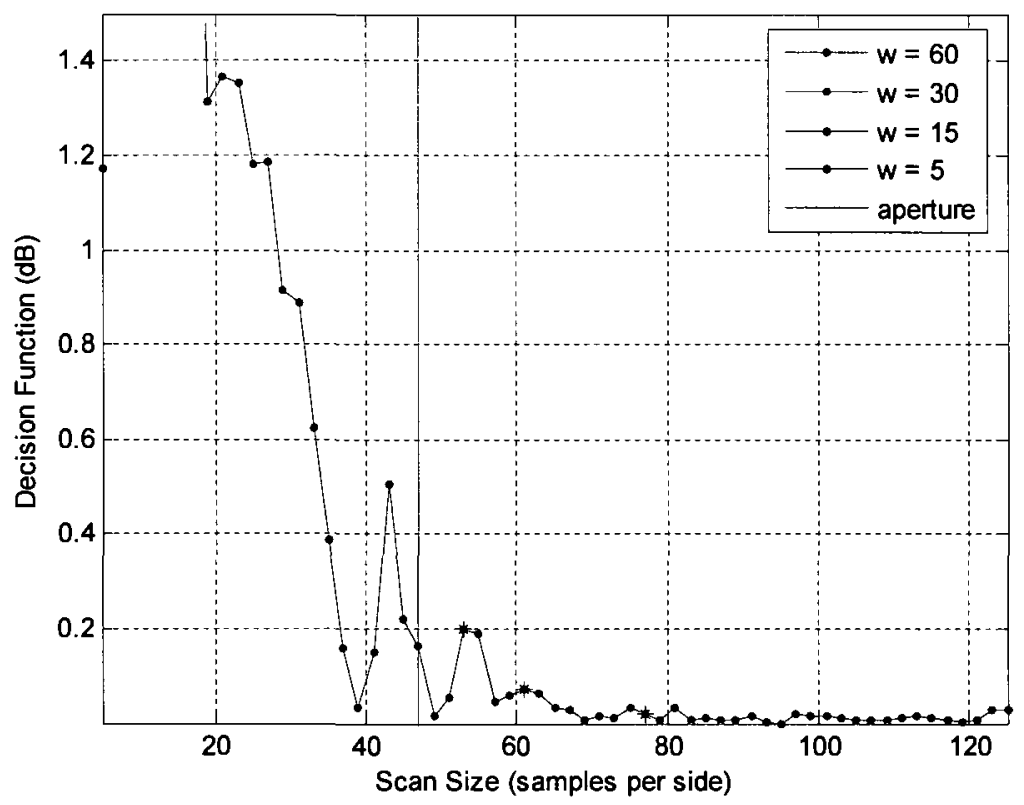

Figure 5.7-6: Cross-polarized component of DFD3 for the offset reflector antenna

\subsubsection{Analysis of Cross-Polarized Decision Function DFNn}

Figure 5.7-7 to Figure 5.7-9 show the $\mathrm{x}$-component of the DFN $n$ decision functions. The $\mathrm{x}$-component of DFN1 and DFN2 are higher than the principle component (y) for the offset reflector antenna. The difference is most significant between the $\xi=60^{\circ}$ case. The fractional plane wave spectrum error estimate of DFN3 has the same general magnitude for both $\mathrm{x}$ and $\mathrm{y}$ components and although the values do differ, the shape of both are similar. 


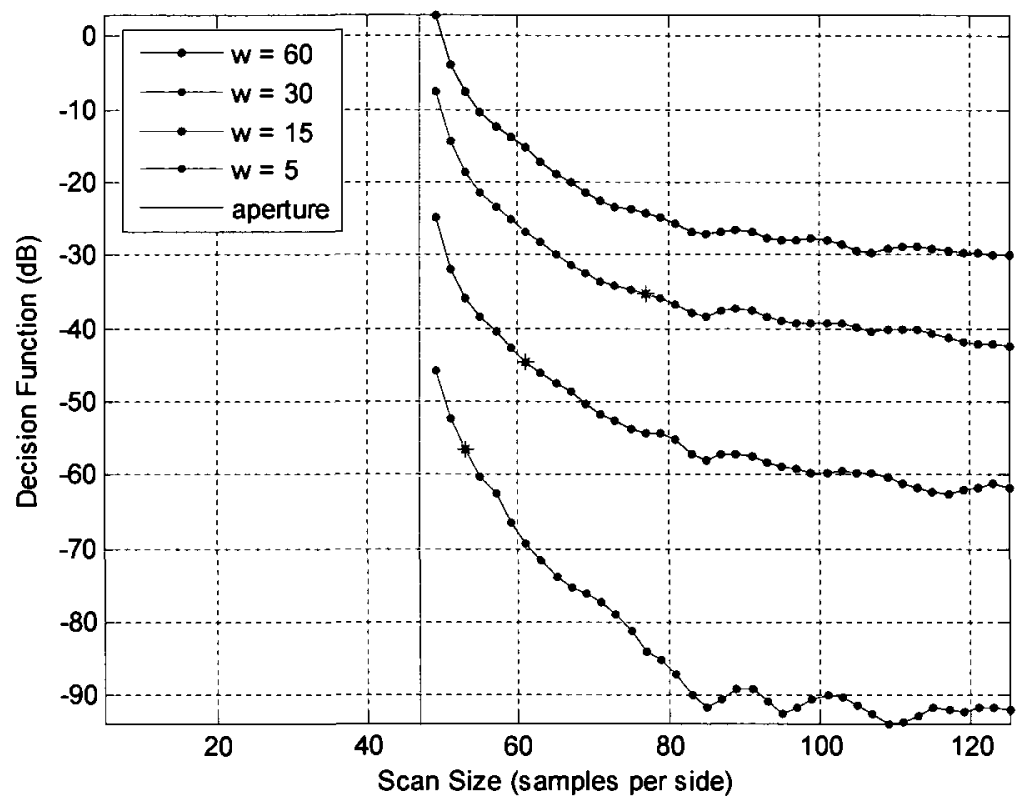

Figure 5.7-7: X-component of DFN1 for the offset reflector antenna

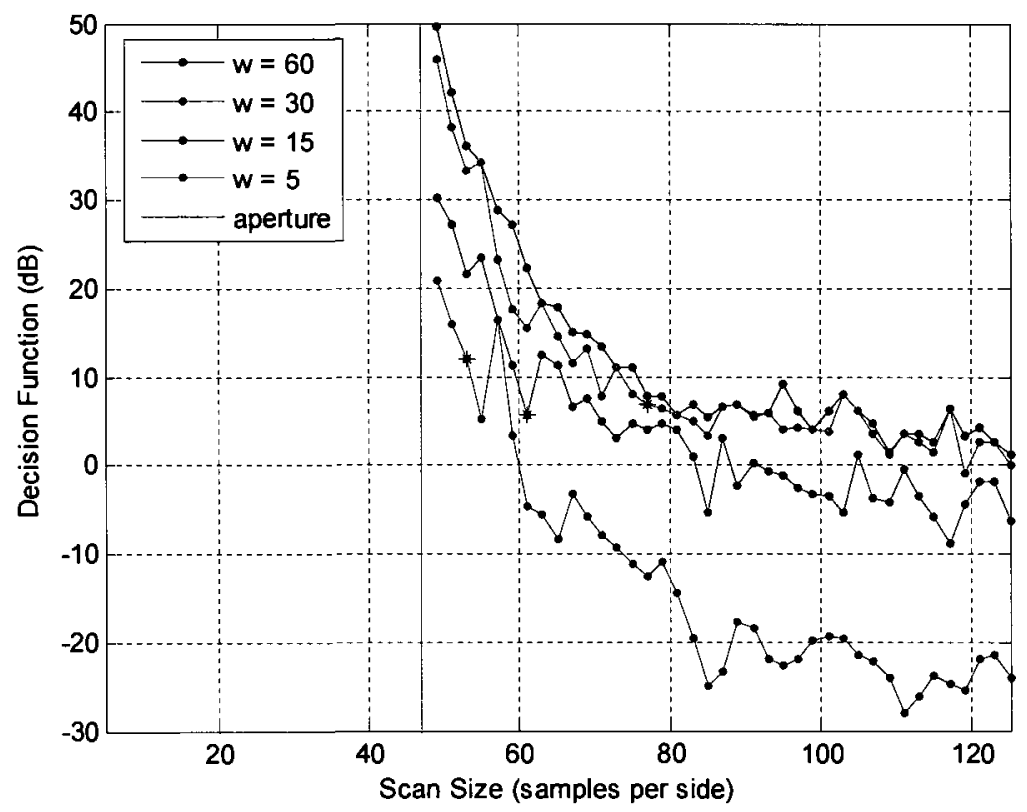

Figure 5.7-8: X-component of DFN2 for the offset reflector antenna 


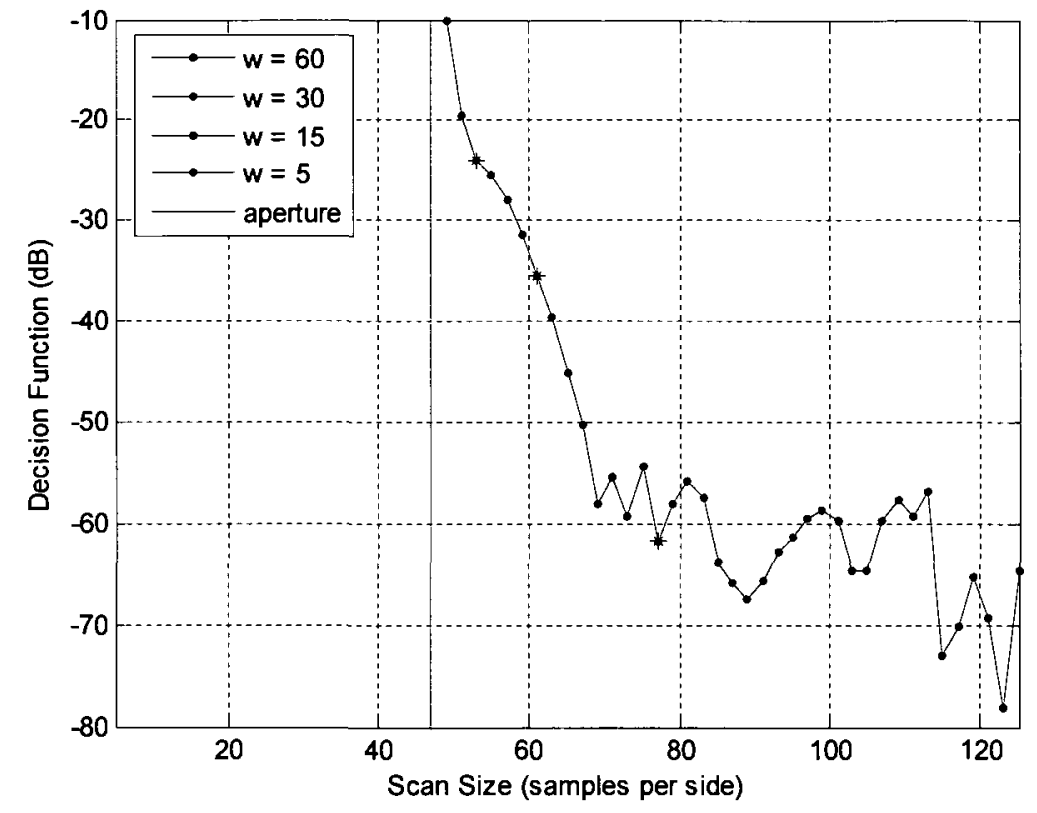

Figure 5.7-9: $X$-component of DFN3 for the offset reflector antenna

\subsection{Conclusions on the Selection of Decision Functions}

In Section 3.3 we identified the properties needed of a decision function. Firstly, it must have a behaviour that allows us to boldly apply a stopping-criterion (that is, take an unambiguous decision that the PNF data acquisition may be terminated). Secondly, it must be such that, when we apply a stopping-criterion, we end up with what we actually want. The experiments conducted in this chapter have identified DFE2, DFD3 and DFN3 as such decision functions. With hindsight we realise that these decision functions are those directly related to specific antenna performance measures, or errors therein. It will thus be possible, no matter how specific an AUT performance specification for a particular application may be (eg. in some applications the root-mean-square sidelobe level is a key requirement), to set up appropriate decision functions and use the adaptive 
PNF approach devised here. In this way, no matter how unique the application, we can acquire the smallest amount of data in order to achieve the desired accuracy of the selected performance measure of the AUT. This will minimise the amount of testing time while simultaneously exhibiting real-time quality assessment of the measurements. At the same time, although the averaging decision functions DFE1, DFD1 and DFN1 do not enable us to take a decision as to the termination of a PNF scan, (and so must be used alongside DFE2, DFD3 and/or DFN3) they nevertheless give and indication of the "overall health" of the measurement as it progresses.

\subsection{References for Chapter 5}

[1] A.Yaghjian. Upper-Bound Errors in Far-Field Antenna Parameters Determined from Planar Near-Field Measurements - Part I, NBS Technical Note 667, USA, 1975. 


\section{CHAPTER 6 - CONCLUSIONS}

\subsection{General Conclusions}

The contributions of this thesis are as follows:

- We have devised the concept of using adaptive techniques in planar near-field measurements. This allows us to reduce test times by only proceeding with PNF scans until the quantities being sought are within the accuracy we require ${ }^{33}$, not more and not less.

- We have defined several possible decision functions for use in the adaptive PNF technique. With modern desktop computers, the far-zone field computation time is negligible compared to the data acquisition time, even if such computation is done repeatedly as part of an adaptive algorithm intent on reducing the amount of data that has to be acquired by continuous monitoring of a set of decision functions whose values have to be computed continually. This is a relatively simple concept, but one that does not appear to have been proposed by others. It provides an uncomplicated way of reducing overall antenna testing time, and can be accomplished with existing PNF scanning systems.

\footnotetext{
${ }^{33}$ There is a satisfying connection here to the quote attributed to Albert Einstein : "Everything should be made as simple as possible, but not one bit simpler".
} 
- We have used these decision functions in a set of experiments (using real nearfield data for actual antennas) which reveal something of the character of these decision functions. These showed that the most useful decision functions are those directly related to antenna performance measures. This fact provides a useful guide to the setting up of decision functions not specifically considered here; we now know that these should be formed from the antenna specifications most important to a particular AUT. This is always possible; if it were not then the performance specifications would not be obtainable from any antenna measurement technique. We envisage a PNF test set-up which displays several decision functions as the data acquisition is proceeding, with flags that indicate when a sufficiently large scan size has been reached to predict a specific performance index.

Although not a key contribution (it was merely a means to an end), we have developed the software $A P N F$ that is able to take any set of raw PNF near-field data (at any iteration) and rapidly compute the far-field patterns and associated decision functions. This would be a useful tool that could be used in a full implementation of the technique. Future work might involve the application of the adaptive approach to cylindrical near-field (CNF) test methods. 


\section{Appendix A: Simpson's Numerical Integration MATLAB}

Function 


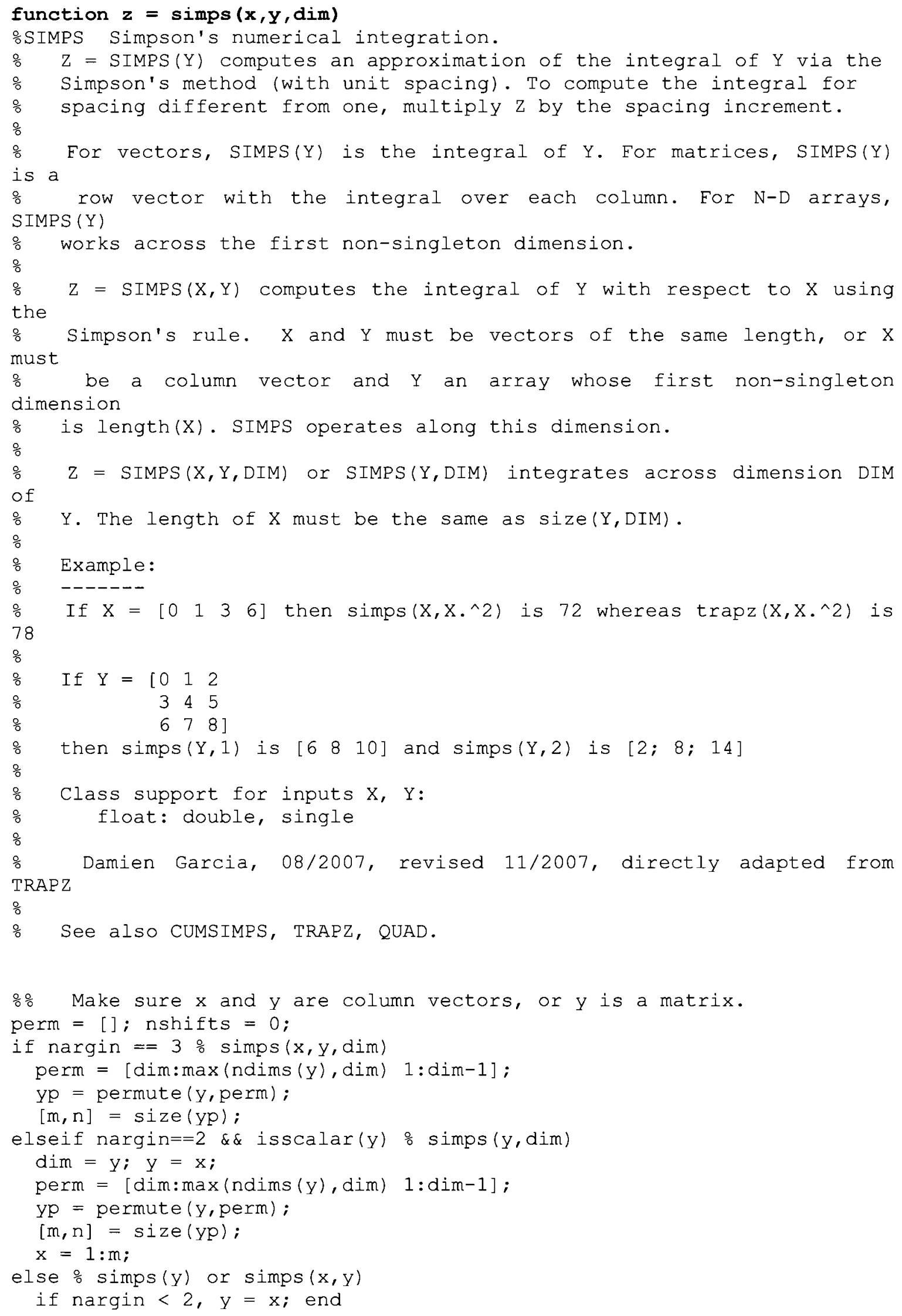




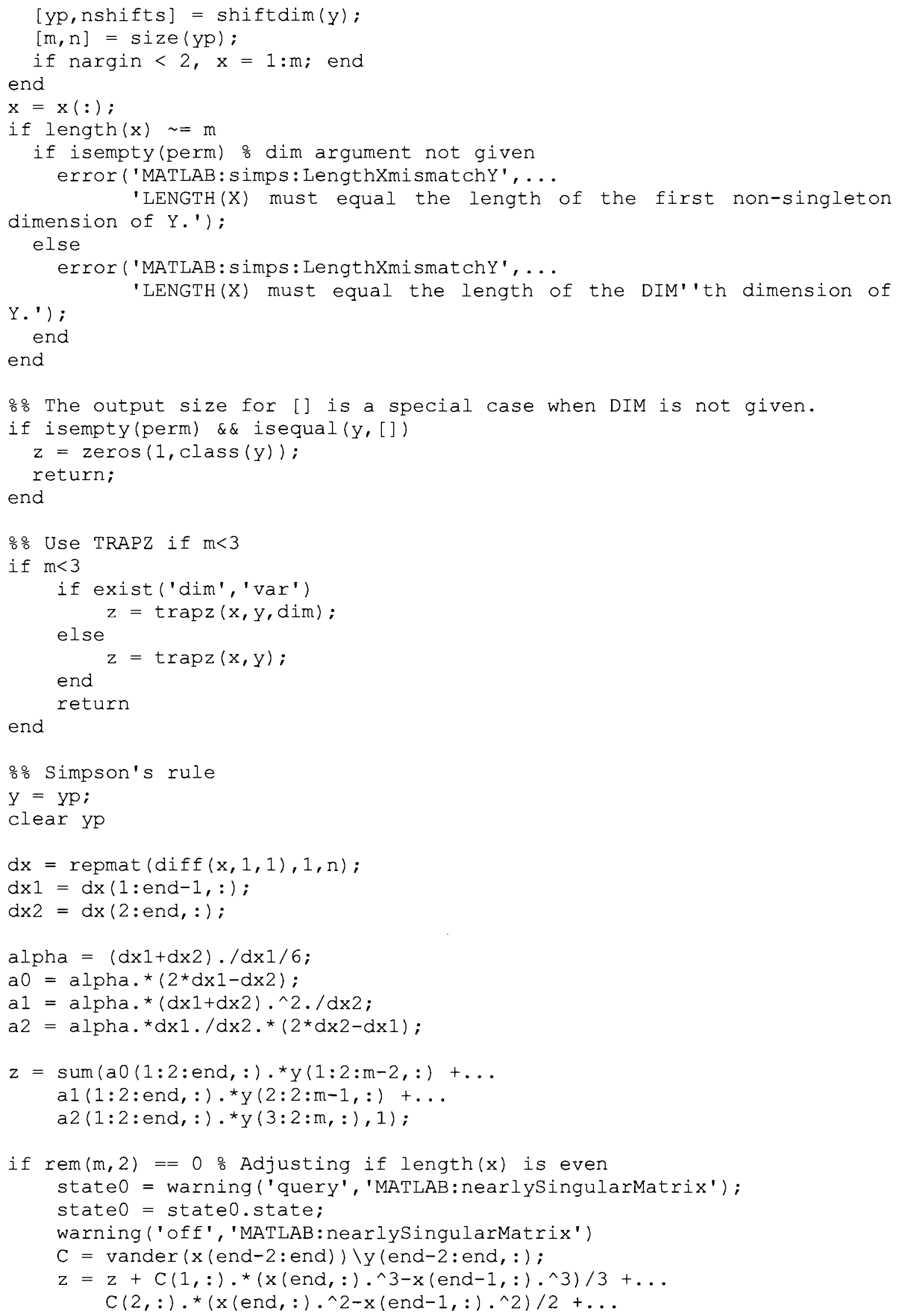


$\mathrm{C}(3,:) .{ }^{\star} \mathrm{dx}($ end, : $)$;

warning (state0, 'MATLAB: nearlySingularMatrix')

end

응 Resizing

$\operatorname{siz}=\operatorname{size}(y) ; \operatorname{siz}(1)=1 ;$

$z=\operatorname{reshape}(z,[$ ones $(1$, nshifts $)$, siz] $) ;$

if $\sim$ isempty (perm), $z=$ ipermute $(z$, perm); end 
Appendix B: Examination of DFN $n$ Nulls 
The following discussion will focus on the $\mathrm{x}$-component of the horn antenna with $\xi=60^{\circ}$ to illustrate the noise-type errors in the evaluation of the contour and surface integrals in the numerator and denominator of expression (3.4.7-1). The evaluation of the contour integral in the numerator is shown in Figure B 1 and the surface integral in the denominator in Figure B 2. There are values in the resulting figures that are much lower than the surrounding values, and magnified view of these noise-type values are shown in Figure B 3 and Figure B 4.

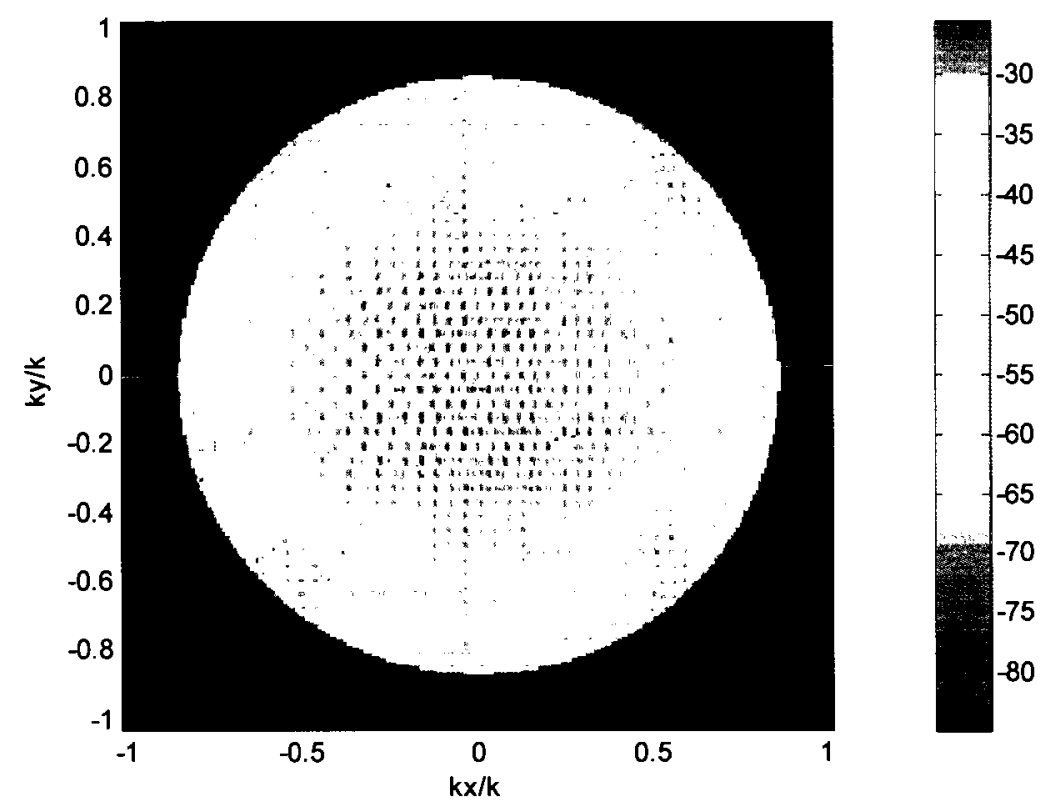

Figure B 1: Evaluation of contour integral of expression (3.4.7-1) for the horn antenna at a scan size of 51 points per side 


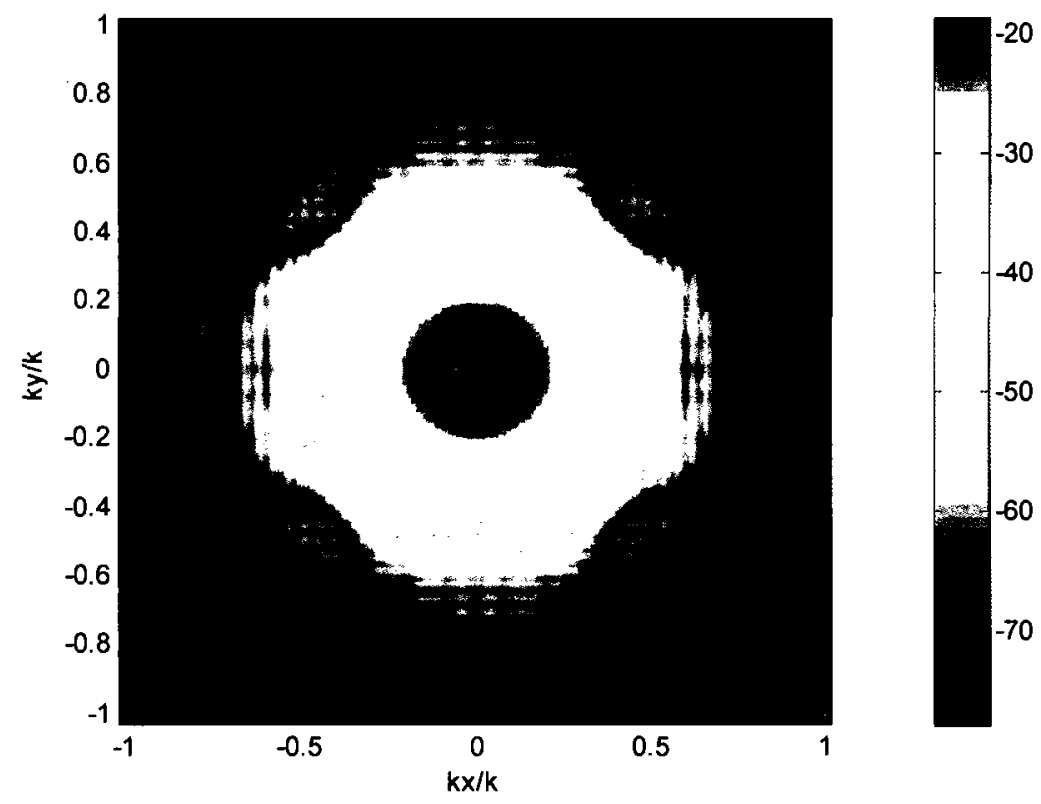

Figure B 2: Evaluation of surface integral of expression (3.4.7-1) for the horn antenna at a scan size of 51 points per side

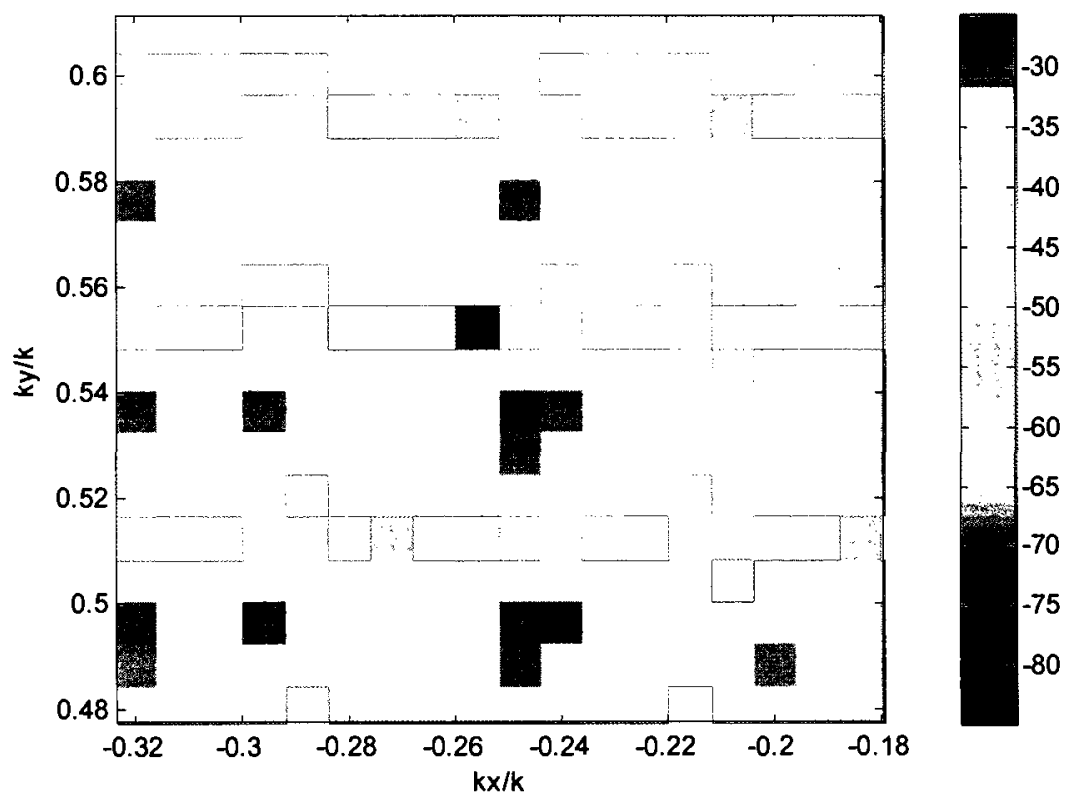

Figure B 3: Magnified view of Figure B 1 showing very low noise-type values that are approximately $30 \mathrm{~dB}$ lower than the surrounding values 


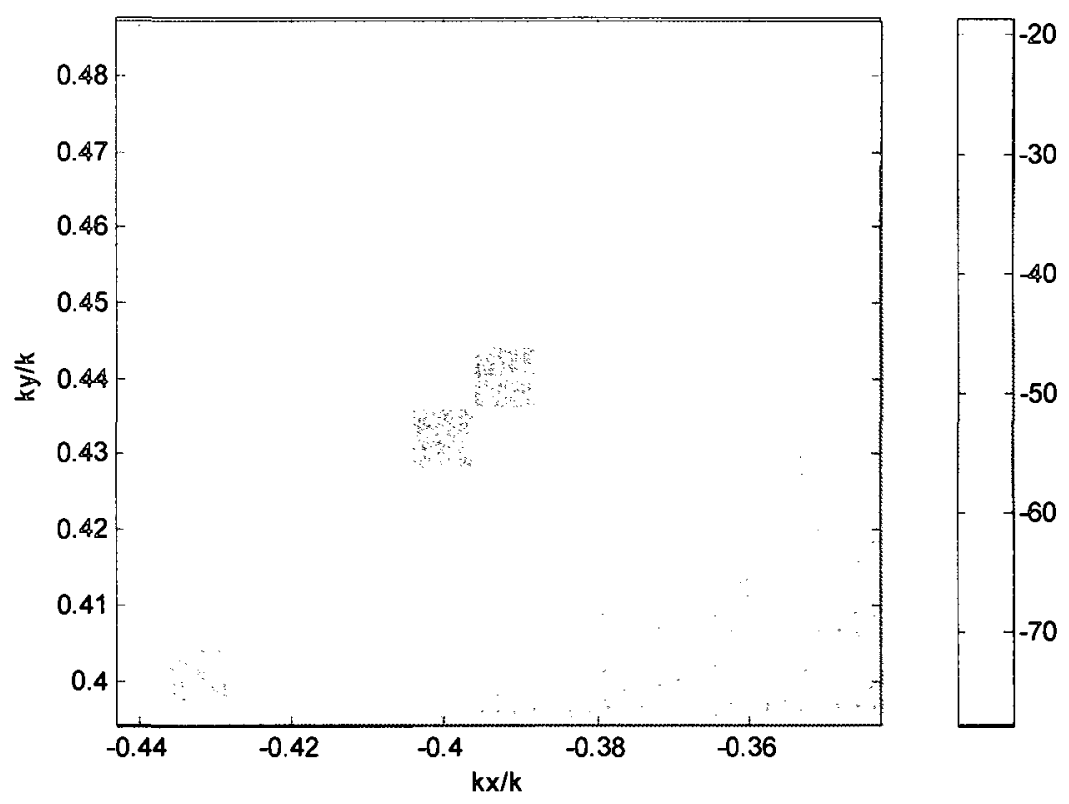

Figure B 4: Magnified view of Figure B 2, showing noise-type values that are approximately $20 \mathrm{~dB}$ lower than the surrounding values

If we apply a lower limit to the evaluation of the above integrals, say $50 \mathrm{~dB}$ below the maximum, we effectively remove these noisy values, as shown by the mask in Figure B 5. This mask was generated by setting the lower limit for the contour integral and combining that mask with one generated from limiting the surface integral with the same threshold. Black areas in the mask are not included in the decision function calculation. It is likely that these noise-type errors are a result of the numerical evaluation of the integrals in expression (3.4.7-1). 


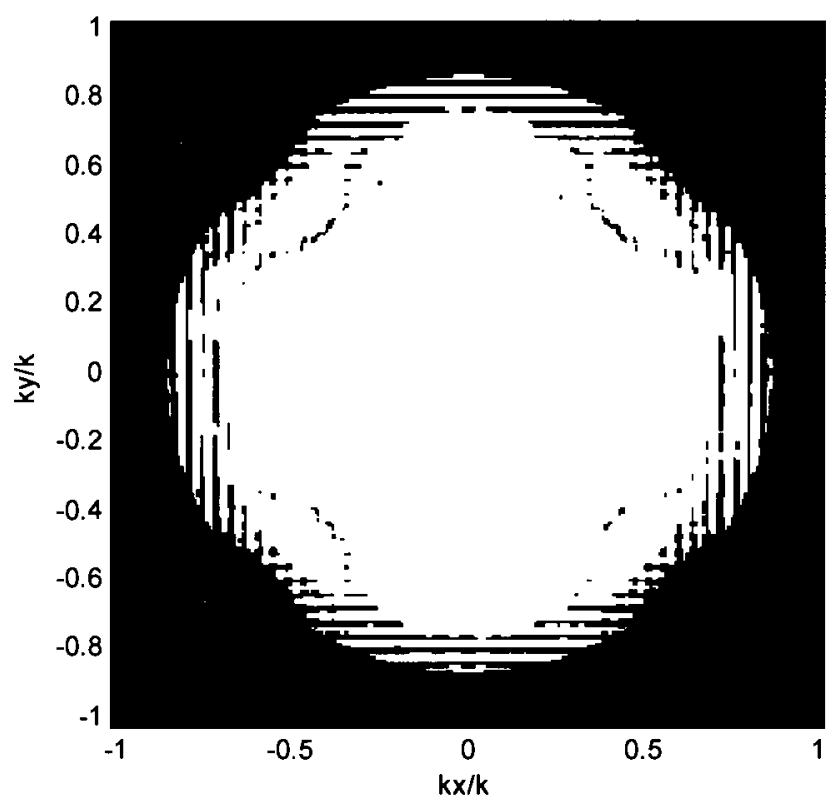

Figure B 5: Mask based on a lower limit set $50 \mathrm{~dB}$ below the maximum of the contour integral and the surface integral. The mask shown here is the combination of the contour integral mask and the surface integral mask. Black areas are not included in the evaluation of the decision function. 